Nevada

Environmental

Restoration

Project

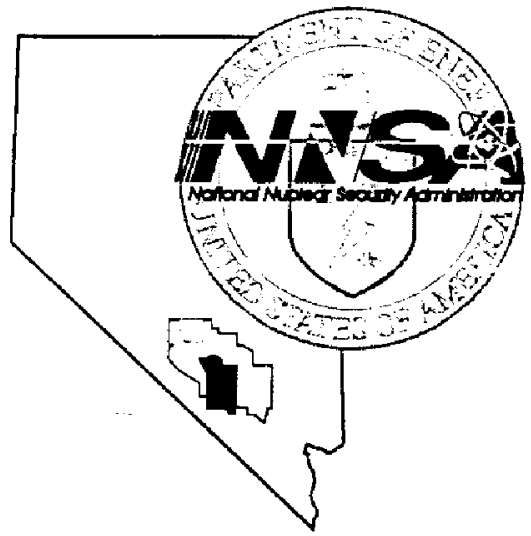

Closure Report for Housekeeping Category Corrective Action Unit 343: Areas 1, 3, \& 4 Housekeeping Sites, Nevada Test Site, Nevada

Controlled Copy No.:

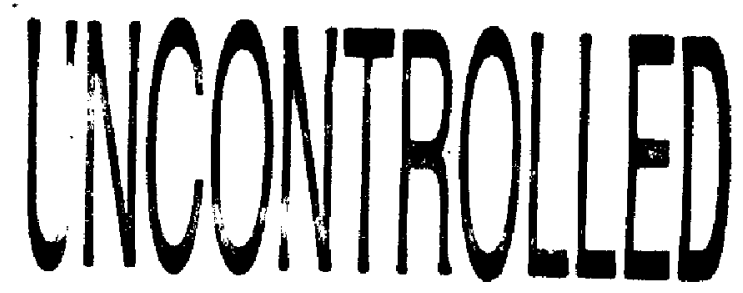

Revision: 0

February 2002

Environmental Restoration

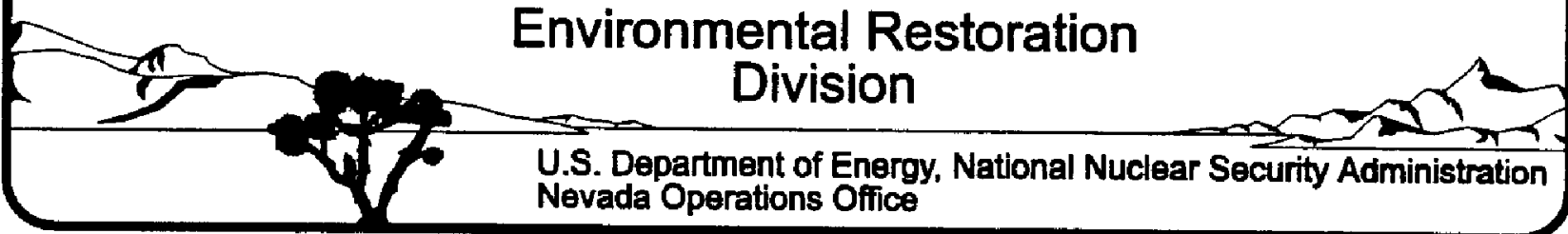




\section{DISCLAIMER STATEMENT}

Reference herein to any specific commercial product, process, or service by trade name, trademark, manufacturer, or otherwise, does not necessarily constitute or imply its endorsement, recommendation, or favoring by the U.S. Government or any agency thereof or its contractors or subcontractors.

\section{AVAILABILITY STATEMENT}

Available for sale to the public from-

U.S. Department of Commerce

National Technical Information Service

5285 Port Royal Road

Springfield, VA 22161-0002

Telephone: 800.553 .6847

Fax: 703.605.6900

E-mail: orders@ntis.fedworld.gov

Online ordering: http:/www.ntis.gov/ordering.htm

Available electronically at http://www.doe.gov/bridge

Available for a processing fee to U.S. Department of Energy and its contractors, in paper, fromU.S. Department of Energy

Office of Scientific and Technical Information

P.O. Box 62

Oak Ridge, TN $37831-0062$

Telephone: 865.576 .8401

Fax: 865.576.5728

E-mail: reports@adonis.osti.gov 


\title{
CLOSURE REPORT FOR HOUSEKEEPING CATEGORY CORRECTIVE ACTION UNIT 343: AREAS 1, 3, \& 4 HOUSEKEEPING SITES, NEVADA TEST SITE, NEVADA
}

\author{
Prepared for: \\ U.S. Department of Energy \\ National Nuclear Security Administration \\ Nevada Operations Office \\ Work Performed Under Contract No. DE-AC08-96-NV11718
}

Controlled Copy No:

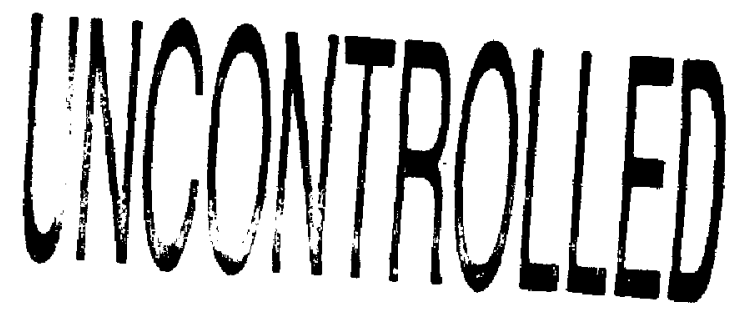

Revision: 0

February 2002 
THIS PAGE INTENTIONALLY LEFT BLANK

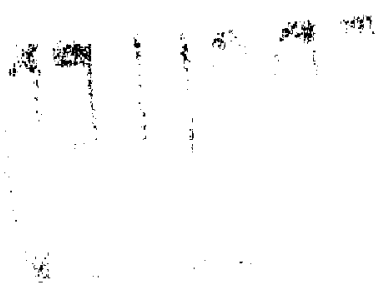




\section{CLOSURE REPORT \\ FOR HOUSEKEEPING CATEGORY \\ CORRECTIVE ACTION UNIT 343: AREAS 1, 3, \& 4 HOUSEKEEPING SITES, NEVADA TEST SITE, NEVADA}
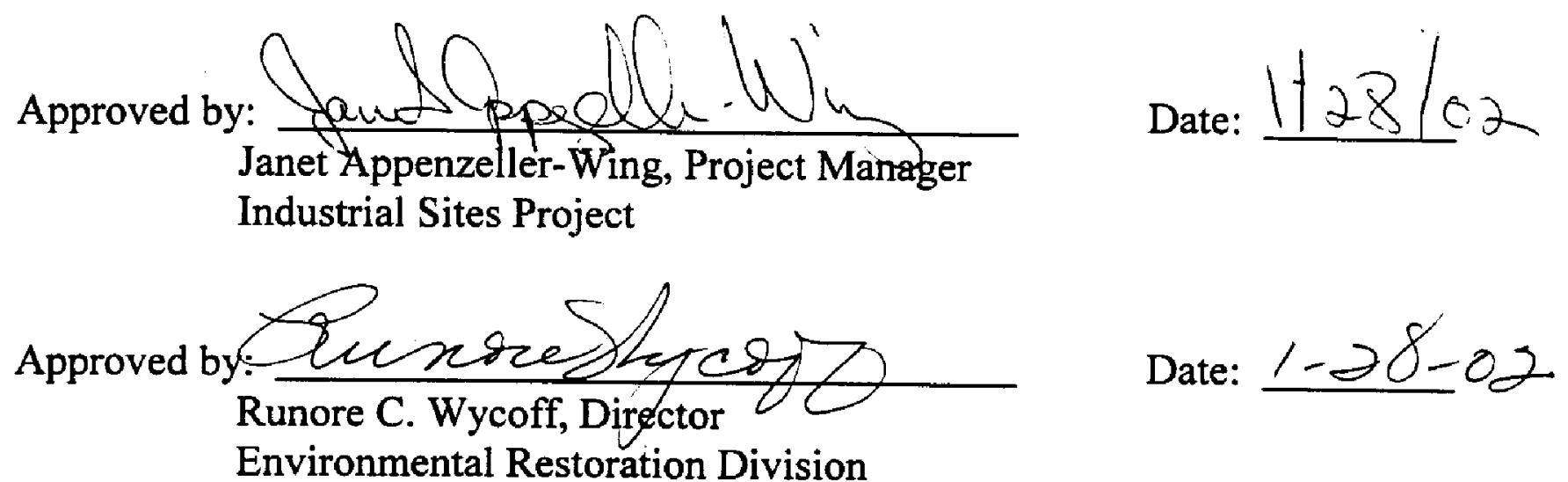
THIS PAGE INTENTIONALLY LEFT BLANK 


\section{TABLE OF CONTENTS}

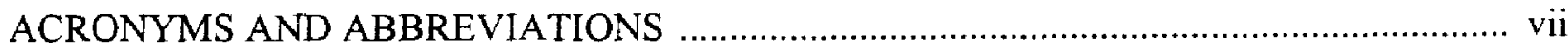

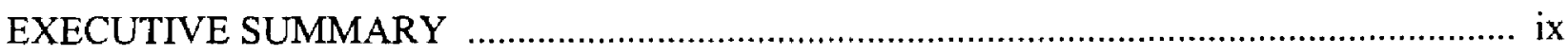

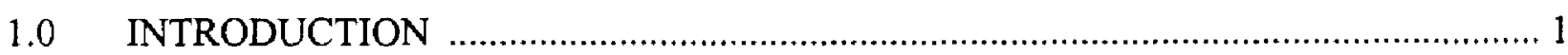

2.0 CLOSURE ACTIVITIES .................................................................................. 7

2.1 DESCRIPTION OF CLOSURE ACTIVITIES ….................................... 7

2.1.1 Preplanning and Site Preparation …................................................... 7

2.2 WASTE CHARACTERIZATION ACTIVITIES ......................................... 7

2.2.1 CAS 01-22-06 Waste Characterization .............................................. 7

2.2.2 CAS 01-24-03 Waste Characterization ….......................................... 10

2.2.3 CAS 03-14-05 Waste Characterization ….......................................... 10

2.2.4 CAS 03-22-06 Waste Characterization .............................................. 10

2.2.5 CAS 03-22-07 Waste Characterization .............................................. 10

2.2.6 CAS 03-22-10 Waste Characterization ............................................... 11

2.2.7 CAS 03-22-12 Waste Characterization .............................................. 11

2.2.8 CAS 03-22-14 Waste Characterization ................................................ 11

2.2.9 CAS 03-22-24 Waste Characterization .................................................. 11

2.2.10 CAS 03-22-25 Waste Characterization ............................................. 12

2.2.11 CAS 03-22-26 Waste Characterization ............................................... 12

2.2.12 CAS 03-22-30 Waste Characterization ............................................. 12

2.2.13 CAS 03-22-34 Waste Characterization ............................................... 12

2.2.14 CAS 03-22-37 Waste Characterization ............................................. 12

2.2.15 CAS 03-24-08 Waste Characterization ............................................. 13

2.2.16 CAS 03-99-10 Waste Characterization ................................................. 13

2.3 SITE CLOSURE ACTIVITIES …....................................................... 13

2.3.1 CAS 01-24-03: Batteries (2) ….................................................... 13

2.3.2 CAS 03-14-05: Transformers; Switch Boxes ....................................... 13

2.3.3 CAS 03-22-06: Drums; Bucket; Can .................................................. 14

2.3.4 CAS 03-22-07: Buckets (2) ......................................................... 14

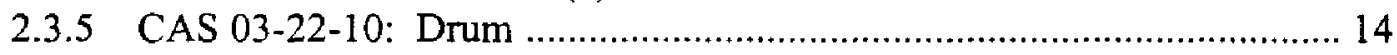

2.3.6 CAS 03-22-12: Drum ................................................................... 14

2.3.7 CAS 03-22-14: Drums (2) .............................................................. 18

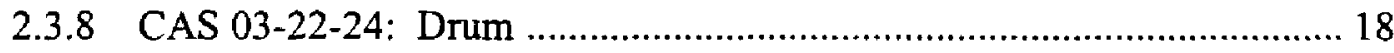

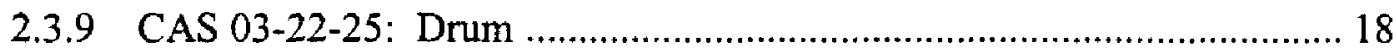

2.3.10 CAS 03-22-26: Can ....................................................................... 18

2.3.11 CAS 03-22-30: Buckets; Cans; Debris ............................................. 19

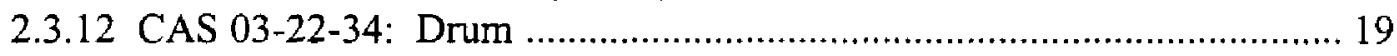

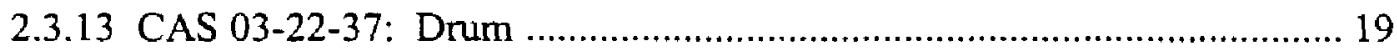

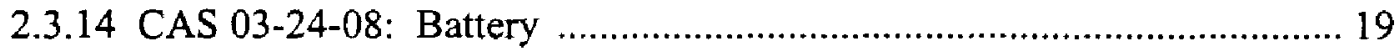

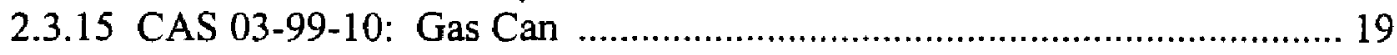

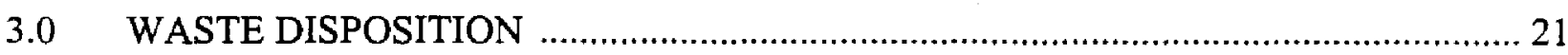




\section{TABLE OF CONTENTS (continued)}

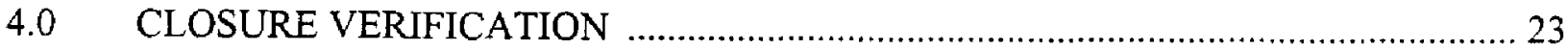

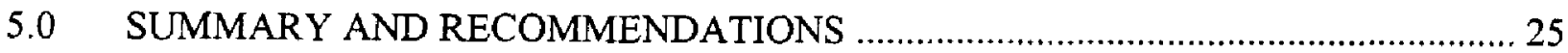

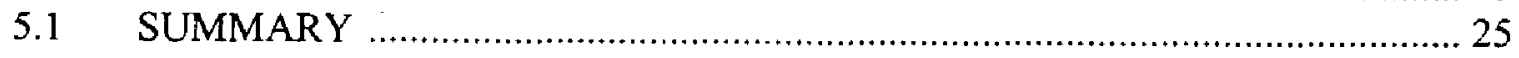

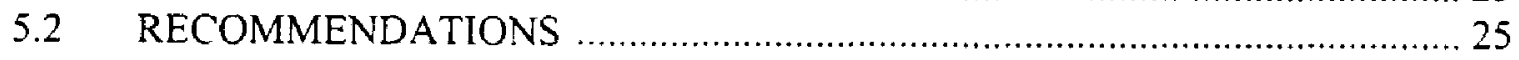

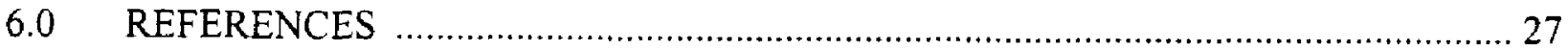

\section{APPENDICES}

APPENDIX A: VERIFICATION SAMPLE ANALYTICAL RESULTS

APPENDIX B: SECTORED HOUSEKEEPING SITE CLOSURE VERIFICATION FORMS

APPENDIX C: CAS SPECIFIC SAMPLING AND ANALYSIS PLAN

APPENDIX D: COMMENT RESPONSE DOCUMENTATION

DISTRIBUTION LIST

\section{FIGURES}

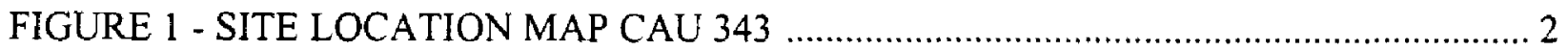

FIGURE 2 - SITE LOCATION MAP CAU 343, AREA 1 SITES ......................................... 3

FIGURE 3 - SITE LOCATION MAP, CAU 343, AREA 3 SITES .................................... 4

FIGURE 4 - SITE LOCATION MAP, CAU 343, AREA 4 SITE .......................................... 5

\section{TABLES}

TABLE 1 - SUMMARY OF ANALYTICAL RESULTS FOR WASTE CHARACTERIZATION SOIL SAMPLES

TABLE 2 - SUMMARY OF ANALYTICAL RESULTS FOR TCLP METALS-LEAD AND $\mathrm{pH}$ IN VERIFICATION SOIL SAMPLES

TABLE 3 - SUMMARY OF ANALYTICAL RESULTS FOR SVOCS AND VOCs IN VERIFICATION SOIL SAMPLES

TABLE 4 - SUMMARY OF ANALYTICAL RESULTS FOR TOTAL PETROLEUM HYDROCARBONS IN VERIFICATION SOIL SAMPLES 


\section{ACRONYMS AND ABBREVIATIONS}

$\mathrm{BN}$

Bechtel Nevada

CAS Corrective Action Site(s)

CAU Corrective Action Unit

CFR Code of Federal Regulations

COC constituent(s) of concern

DOE/NV U.S. Department of Energy, Nevada Operations Office

EPA U.S. Environmental Protection Agency

ER Environmental Restoration

FFACO Federal Facility Agreement and Consent Order

ICP Inductively Coupled Plasma

IT International Technology Corporation

m meter (s)

$\mathrm{mg} / \mathrm{kg} \quad$ milligram(s) per kilogram

NAC Nevada Administrative Code

ND Not Detected

NDEP Nevada Division of Environmental Protection

NNSA/NV U.S. Department of Energy, National Nuclear Security Administration Nevada Operations Office

NTS Nevada Test Site

PCBs polychlorinated biphenyls

RCRA Resource Conservation and Recovery Act

PRG preliminary remediation goal

RSM RAD Safe Marker

SVOC semivolatile organic compound

TPH total petroleum hydrocarbon

VOC volatile organic compound

XRF X-ray Fluorescence Device 
CLOSURE REPORT - CAU 343

Areas $1,3, \& 4$ Housekeeping Sites Section: Contents Revision: 0

Date: February 2002

\section{THIS PAGE INTENTIONALLY LEFT BLANK}




\section{EXECUTIVE SUMMARY}

The 18 Corrective Action Sites (CAS) that comprise Corrective Action Unit (CAU) 343 were closed by the following actions:

- CAS 01-22-06: Closed with no action required.

- CAS 01-24-03: Clean closed.

- CAS 01-99-01: Moved to CAU 357.

- CAS 03-14-05: Clean closed.

- CAS 03-22-06: Clean closed.

- CAS 03-22-07: Clean closed.

- CAS 03-22-10: Clean closed.

- CAS 03-22-12: Clean closed.

- CAS 03-22-14: Clean closed.

- CAS 03-22-24: Clean closed.

- CAS 03-22-25: Clean closed

- CAS 03-22-26: Clean closed.

- CAS 03-22-30: Clean closed.

- CAS 03-22-34: Clean closed.

- CAS 03-22-37: Clean closed.

- CAS 03-24-08: Clean closed.

- CAS 03-99-10: Clean closed.

- CAS 04-26-03: Moved to CAU 357. 
CLOSURE REPORT - CAL 343

Areas 1, 3, \& 4 Housekeeping Sites Section: Contents

Revision: 0 Date: February 2002

\section{THIS PAGE INTENTIONALLY LEFT BLANK}




\subsection{INTRODUCTION}

This Closure Report documents the closure activities conducted for CAU 343: Areas 1, 3, \& 4 Housekeeping Sites. CAU 343 is listed in Appendix III of the Federal Facility Agreement and Consent Order (FFACO) (FFACO, 1996) and consists of the following 18 Corrective Action Sites (CASs) located in Areas 1, 3, and 4 of the Nevada Test Site (NTS) (Figures 1, 2, 3, and 4):

- $\quad$ CAS 01-22-06: Drums (2)
- $\quad$ CAS 01-24-03: Batteries (2)
- $\quad$ CAS 01-99-01: Boxes; Pipes
- $\quad$ CAS-14-05: Transformers; Switch Boxes
- $\quad$ CAS 03-22-06: Drums; Bucket; Can
- $\quad$ CAS 03-22-10: Drum
- $\quad$ CAS 03-22-12: Drum
- $\quad$ CAS 03-22-14: Drums (2)
- $\quad$ CAS 03-22-24: Drum
- $\quad$ CAS 03-22-25: Drum
- $\quad$ CAS 03-22-30: Buckets; Cans; Debris
- $\quad$ CAS 03-22-34: Drum
- $\quad$ CAS 03-24-08: Drum
- $\quad$ CAS 03-99-10: Gas Can
CAS 04-26-03: Lead Bricks

Chosure activities were performed in two phases. Phase I activities consisted of collecting waste characterization samples of soil at appropriate sites. The results were used to determine how waste generated during closure activities would be handled and disposed (i.e., as nonhazardous sanitary or hazardous waste). Phase 2 activities consisted of closing each CAS by removing debris and/or material, disposing of the generated waste, and verifying that each site was cleanclosed by visual inspection and/or collecting soil verification samples for laboratory analysis.

One site (CAS 01-22-06) was closed with no further action. This determination was made after reviewing the Preliminary Assessment Database, which mentioned that the drums have already been removed. During several Bechtel Nevada Environmental Restoration (BN/ER) site visits, there were three pieces of scrap metal located that were previously mistaken as being remnants of drums. Additionally, determination of a no action closure was made after concurrence with Nevada Division of Environmental Protection (NDEP). Two other sites (CAS 01-99-01 and CAS 04-26-03) were moved into CAU 357 in Appendix III of the FFACO because the housekeeping process was determined not to be adequate to close them.

Copies of the analytical results for the site verification samples are included in Appendix A. Copies of the Sectored Housekeeping Site Closure Verification Forms for each of the 15 CASs are included in Appendix B. 
Date: February 2002

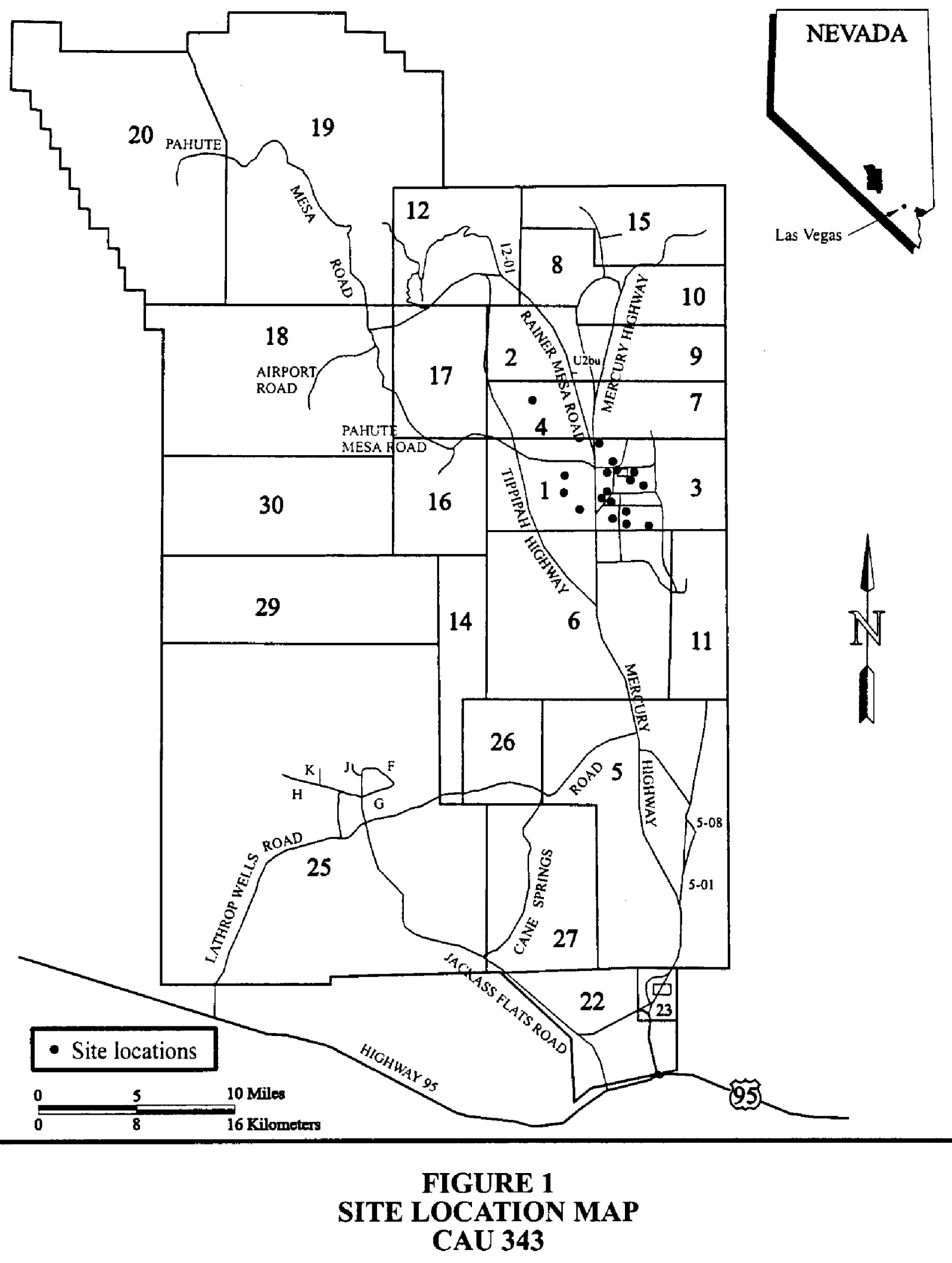


Date: February 2002

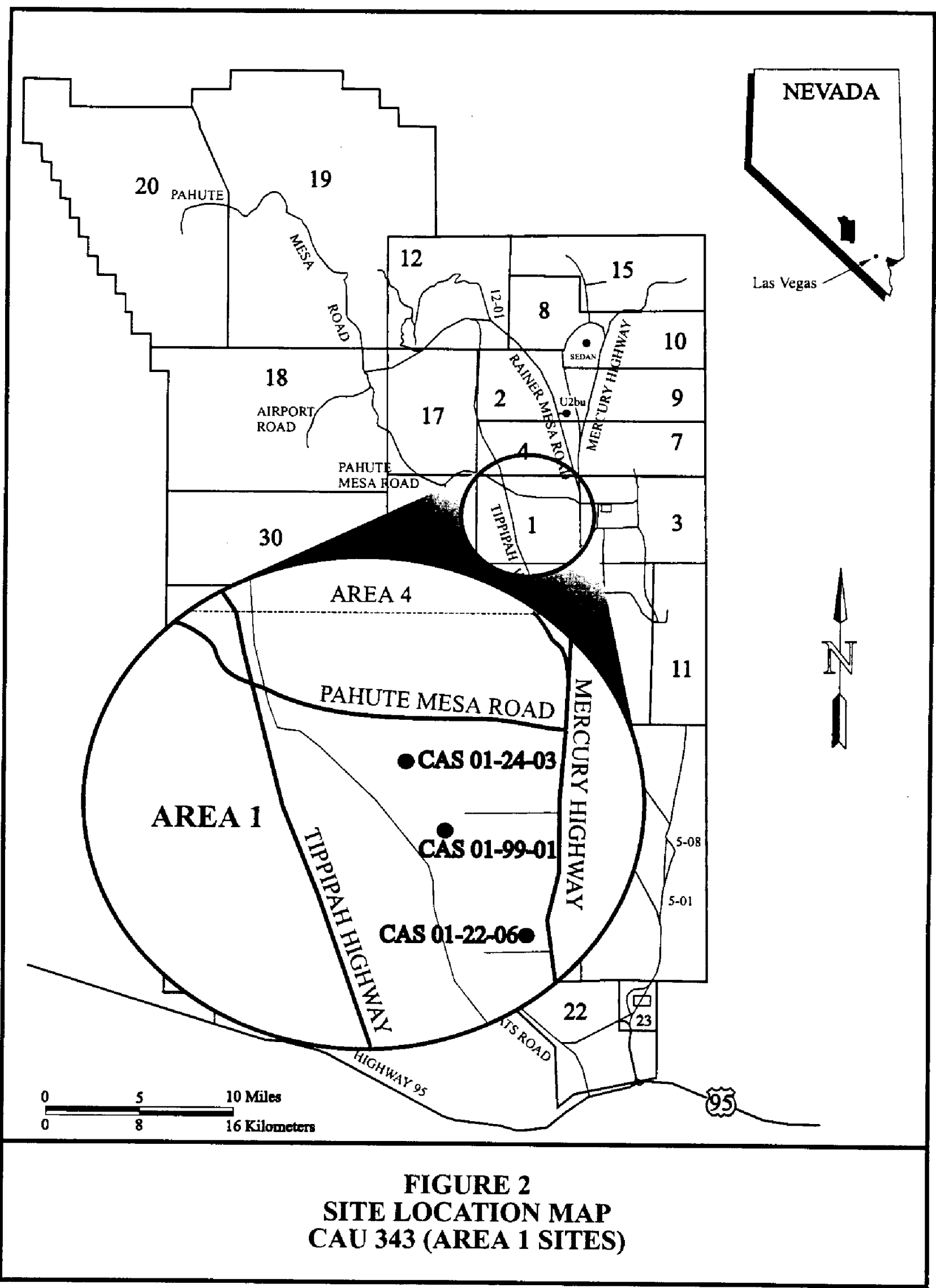




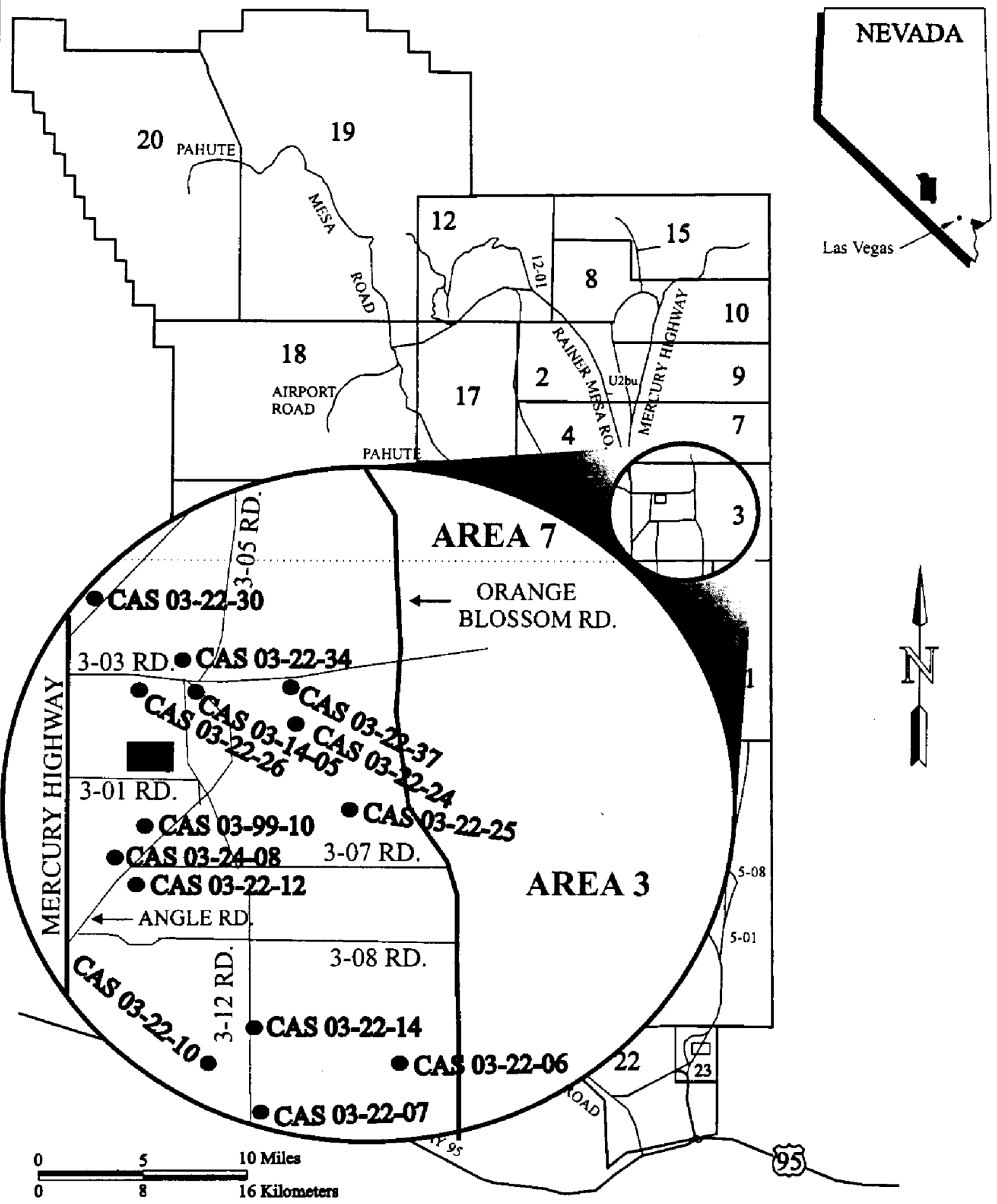

FIGURE 3

SITE LOCATION MAP

CAU 343 (AREA 3 SITES) 
Date: February 2002

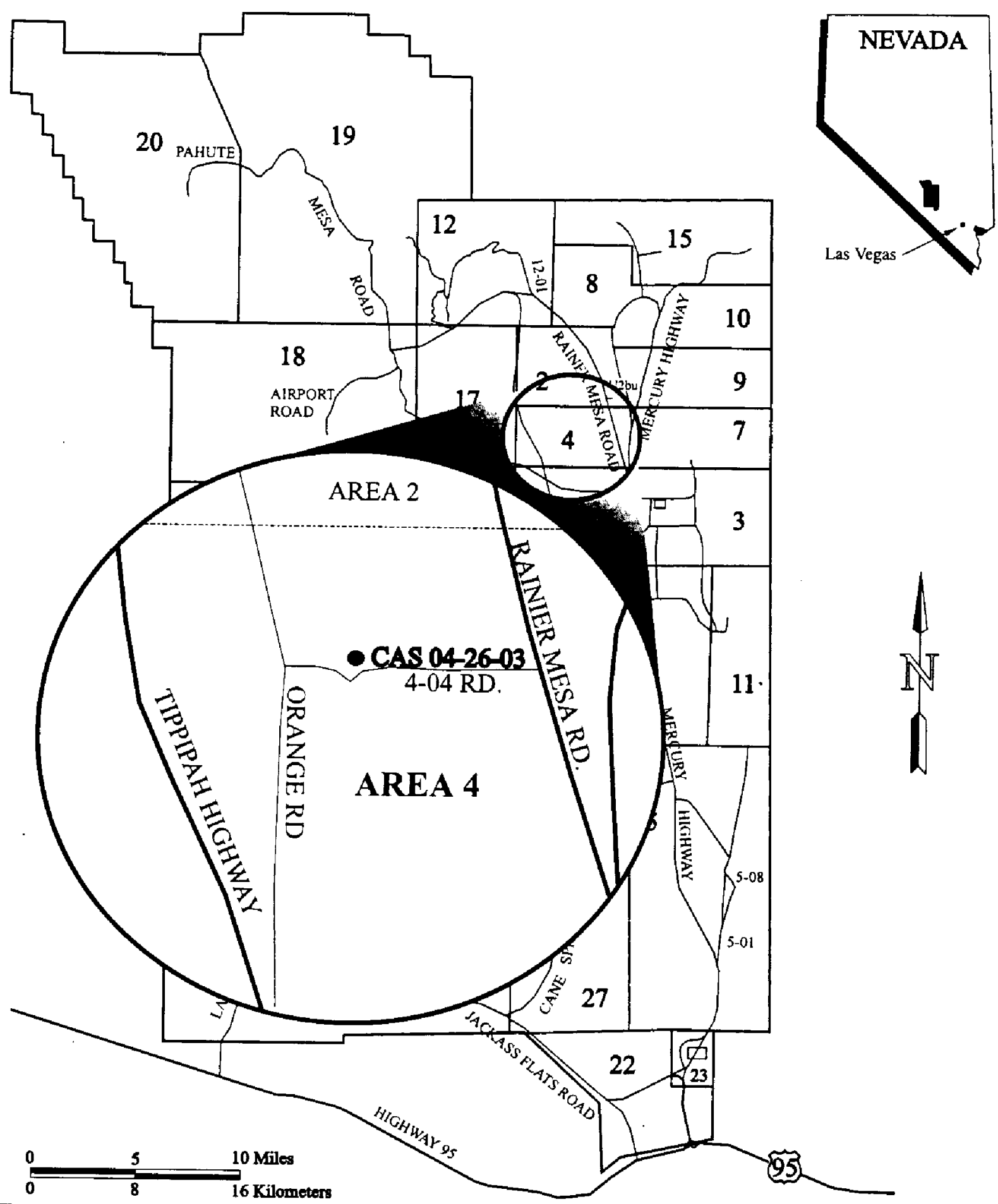

FIGURE 4

SITE LOCATION MAP

CAU 343 (AREA 4 SITE) 
CLOSURE REPORT - CAU 343

Areas 1, 3, \& 4 Housekeeping Sites

Section: Introduetion Revision: 0

Date: February 2002

THIS PAGE INTENTIONALLY LEFT BLANK 


\subsection{CLOSURE ACTIVITIES}

This section details the specific corrective action activities completed during the closure of CAU 343: Areas 1, 3, \& 4 Housekeeping Sites. Copies of the analytical data reports for all verification samples are included in Appendix A and copies of the Sectored Housekeeping Site Closure Verification Forms are included in Appendix B.

\subsection{DESCRIPTION OF CLOSURE ACTIVITIES}

\subsubsection{Preplanning and Site Preparation}

Planning documents prepared prior to the beginning of closure activities include the Sectored Clean-up Work Plan For Housekeeping Category Waste Sites (DOE/NV, 2000); Generic Field Management Plan for Housekeeping Category Waste Sites (BN, 2000); Site-Specific Health and Safety Plan for Corrective Action Unit 343: Areas 1, 3, \& 4 Housekeeping Sites (BN, 2001); Site Maintenance Work Packages; and DOE/NV Real Estate/Operation Permit (BN-0179-00). In addition, a National Environmental Policy Act checklist was prepared (NV-00-060) and a preactivity site survey was conducted by a BN biologist. The survey confirmed the absence of sensitive animal or plant species at all 18 CASs. A Readiness Review was held on November 20,2001 , by BN/ER. BN/ER and Site Maintenance personnel held a pre-job field briefing on November 26, 2001, prior to the start of site-closure field activities.

\subsection{WASTE CHARACTERIZATION ACTIVITIES}

At 14 of the $18 \mathrm{CASs}$, samples of soil and/or material were collected and analyzed to characterize the waste that was expected to be generated during site-closure activities. Table 1 presents the analyses that were conducted for waste characterization samples, the results of these analyses, and the resulting waste classifications.

\subsubsection{CAS 01-22-06 Waste Characterization}

The Preliminary Assessment documents confirm that the drums have already been removed. In past BN/ER site visits, only pieces of scrap metal were found in the bottom of the U-1 e crater. From the edge of the crater, the scrap metal appeared to be drum remnants. Upon entering the crater, the scrap metal was determined not to be drum remnants. However, soil samples were collected from underneath the pieces of scrap metal to determine if the soils were impacted.

Nevada Division of Environmental Protection (NDEP) visited the site on November 19, 2001, and verified that no drums were present at the site. NDEP observed that scrap metal was present at the site and was informed by BN/ER that soil samples had been collected at the site. Based on the results of the soil samples collected in the area where drums were previously located, NDEP concurred with the proposal for no further action at this site. On October 9, 2001, the soil samples (012206-0-1, 012206-0-2, and 012206-0-3) were analyzed for total petroleum hydrocarbons (TPH) full scan, total Resource Conservation and Recovery Act (RCRA) metals, 
TABLE 1-SUMMARY OF ANALYTICAL RESULTS FOR WASTE CHARACTERIZATION SOIL SAMPLES

\begin{tabular}{|c|c|c|c|c|}
\hline CAS & $\begin{array}{l}\text { SAMPLE } \\
\text { TYPE }\end{array}$ & ANALYSIS & $\begin{array}{l}\text { ANALYTICAL } \\
\text { RESULTS }\end{array}$ & WASTE TYPE \\
\hline $01-22-06$ & Soil & $\begin{array}{l}\text { TPH full scan }{ }^{\mathrm{a}} \text {, total } \\
\text { RCRA metals } \\
\text {, VOCs } \\
\text { SVOCs }^{\mathrm{d}}, \mathrm{PCB} \mathrm{s}^{\mathrm{e}} \text {, and } \\
\text { gamma spectroscopy }\end{array}$ & $\begin{array}{l}\text { All analyses lower than action } \\
\text { levels. }\end{array}$ & No Action \\
\hline 01-24-03 & Soil & $\begin{array}{l}\text { TCLP metals (lead) and } \\
\mathrm{pH}\end{array}$ & TCLP-lead is $35 \mathrm{mg} / \mathrm{L}$ & $\begin{array}{l}\text { Hazardous waste } \\
\text { (lead) }\end{array}$ \\
\hline 01-99-01 & N/A & $\mathrm{N} / \mathrm{A}$ & N/A & $\begin{array}{l}\text { No Action } \\
\text { Being removed } \\
\text { from CAU } 343\end{array}$ \\
\hline 03-14-05 & $\mathrm{N} / \mathrm{A}$ & N/A & N/A & $\begin{array}{l}\text { Sanitary and } \\
\text { Recyclable }\end{array}$ \\
\hline 03-22-06 & Soil & $\begin{array}{l}\text { TPH full scan, total } \\
\text { RCRA metals, VOCs, } \\
\text { SVOCs, PCBs, and } \\
\text { gamma spectroscopy }\end{array}$ & $\begin{array}{l}\text { All analyses lower than action } \\
\text { levels. }\end{array}$ & Sanitary \\
\hline 03-22-07 & Soil & $\begin{array}{l}\text { TPH full scan, total } \\
\text { RCRA metals, VOCs, } \\
\text { SVOCs, PCBs, and } \\
\text { gamma spectroscopy }\end{array}$ & $\begin{array}{l}\text { Several SVOCs higher than } \\
\text { action levels. } \\
\text { One VOC higher than action } \\
\text { levels. } \\
\text { Full-scan TPH higher than action } \\
\text { levels. }\end{array}$ & Hydrocarbon \\
\hline $03-22-10$ & Soil & $\begin{array}{l}\text { TPH full scan, total } \\
\text { RCRA metals, VOCs, } \\
\text { SVOCs, PCBs, and } \\
\text { gamma spectroscopy }\end{array}$ & All analyses lower than action & Sanitary \\
\hline $03-22-12$ & Soil & $\begin{array}{l}\text { TPH full scan, total } \\
\text { RCRA metals, VOCs, } \\
\text { SVOCs, PCBs, and } \\
\text { gamma spectroscopy }\end{array}$ & $\begin{array}{l}\text { Full-scan TPH higher than action } \\
\text { levels }\end{array}$ & Hydrocarbon \\
\hline $03-22-14$ & Soil & $\begin{array}{l}\text { TPH full scan, total } \\
\text { RCRA metals, VOCs, } \\
\text { SVOCs, PCBs, and } \\
\text { gamma spectroscopy }\end{array}$ & $\begin{array}{l}\text { One SVOC higher than action } \\
\text { levels. } \\
\text { Full-scan TPH higher than action } \\
\text { levels. }\end{array}$ & Hydrocarbon \\
\hline
\end{tabular}




\section{TABLE 1-SUMMARY OF ANALYTICAL RESULTS FOR WASTE CHARACTERIZATION SOIL SAMPLES (continued)}

\begin{tabular}{|c|c|c|c|c|}
\hline CAS & $\begin{array}{l}\text { SAMPLE } \\
\text { TYPE }\end{array}$ & ANALYSIS & $\begin{array}{c}\text { ANALYTICAL } \\
\text { RESULTS }\end{array}$ & WASTE TYPE \\
\hline $03-22-24$ & Soil & $\begin{array}{l}\text { TPH full scan, total } \\
\text { RCRA metals, VOCs, } \\
\text { SVOCs, PCBs, and } \\
\text { gamma spectroscopy }\end{array}$ & All analyses lower than action & Sanitary \\
\hline $03-22-25$ & Soil & $\begin{array}{l}\text { Total RCRA metals, } \\
\text { pH, and gamma } \\
\text { spectroscopy }\end{array}$ & $\mathrm{pH}$ is higher than action levels & Sanitary \\
\hline $03-22-26$ & Soil & $\begin{array}{l}\text { TPH full scan, total } \\
\text { RCRA metals, VOCs, } \\
\text { SVOCs, PCBs, and } \\
\text { gamma spectroscopy }\end{array}$ & $\begin{array}{l}\text { All analyses lower than action } \\
\text { levels. }\end{array}$ & Sanitary \\
\hline $03-22-30$ & N/A & $\mathrm{N} / \mathrm{A}$ & $\mathrm{N} / \mathrm{A}$ & Sanitary (debris) \\
\hline $03-22-34$ & Soil & $\begin{array}{l}\text { TPH full scan, total } \\
\text { RCRA metals, VOCs, } \\
\text { SVOCs, PCBs, and } \\
\text { gamma spectroscopy }\end{array}$ & $\begin{array}{l}\text { All analyses lower than action } \\
\text { levels. }\end{array}$ & Sanitary \\
\hline $03-22-37$ & Soil & $\begin{array}{l}\text { TPH full scan, total } \\
\text { RCRA metals, VOCs, } \\
\text { SVOCs, PCBs, and } \\
\text { gamma spectroscopy }\end{array}$ & $\begin{array}{l}\text { All analyses lower than action } \\
\text { levels. }\end{array}$ & Sanitary \\
\hline 03-24-08 & Soil & $\begin{array}{l}\text { TCLP metals (lead) and } \\
\mathrm{pH}\end{array}$ & All analyses lower than action & Recyclable \\
\hline $03-99-10$ & Soil & $\begin{array}{l}\text { TPH (gasoline) and } \\
\text { TCLP metals (lead) }\end{array}$ & $\begin{array}{l}\text { All analyses lower than action } \\
\text { levels. }\end{array}$ & Sanitary \\
\hline 04-26-03 & N/A & $\mathrm{N} / \mathrm{A}$ & $\mathrm{N} / \mathrm{A}$ & $\begin{array}{l}\text { No Action } \\
\text { Being removed } \\
\text { from CAU } 343\end{array}$ \\
\hline
\end{tabular}

Total petroleum hydrocarbons (TPH) analysis performed by U.S. Environmental Protection Agency (EPA) Method SW-846 8015M (EPA, 1996b). See analytical reports in Appendix A for detection limits.

"Resource Conservation and Recovery Act (RCRA) metals analysis performed by EPA Method SW-846 6010, 7471A (EPA, $1996 \mathrm{~b}$ ).

'Volatile organic compounds (VOC) analysis performed by EPA Method SW-846 8260B (EPA, 19966). See analytical reports in Appendix A for detection limits.

"Semivolatile organic compounds (SVOC) analysis performed by EPA Method SW-846 $8270 \mathrm{C}$ (EPA, 1996b). See analytical reports in Appendix A for detection limits.

'Polychlorinated biphenyls (PCBs) analysis performed by EPA Method SW-846 8082 (EPA, 1996b). See analytical reports in Appendix A for detection limits.

'Gamma spectroscopy analysis performed by EPA Method SW-846 901.1 (EPA, 1996b). See analytical reparts in Appendix A for detection limits. 
polychlorinated biphenyls (PCBs), volatile organic compounds (VOCs), semi-volatile organic compounds (SVOCs), and gamma spectroscopy. Waste characterization sampling confirms that no constituents of concem (COCs) are present. The site was left with no further action because the drums have been documented as already being removed and the pieces of scrap metal at the bottom of the U-1e crater pose no threat to the environment.

\subsubsection{CAS 01-24-03 Waste Characterization}

Waste generated at this site included a small area that included broken pieces of a lead battery and a pile of approximately 30 to 4050 -caliber brass shells. On October 9, 2001, the soil sample (012403-0-1) was collected from the surface within the area of the broken pieces of battery and analyzed for TCLP metals-lead and soil pH. Results for TCLP metals-lead were higher than the Region 9 Preliminary Remediation Goals (PRGs) (EPA, 1996a) for industrial soils and was determined to be hazardous waste from Title 40 Code of Federal Regulations (CFR) 261.24 Table 1 (EPA, 1996c). The soil underneath the broken pieces of battery was sampled to determine if the pieces impacted the soil underneath. Based on waste characterization results, the lead-contaminated soil was categorized as hazardous waste. The 50-caliber brass shells were disposed of as sanitary waste.

\subsubsection{CAS 03-14-05 Waste Characterization}

Waste generated at this site included two transformers (non-PCB) and oils from two switches. No waste characterization sampling was required at this site because the transformers were dry and the switches were intact and contained in a concrete vault. The two transformers were free to be released as sanitary waste. The oils drained from the switches were recycled by the Site Services Department.

\subsubsection{CAS 03-22-06 Waste Characterization}

Waste generated at this site included two small piles of magnetite and associated soil. A magnet was used in the field to confirm that the material was magnetite. On October 11,2001, the soil samples (032226-0-0 and 032226-0-1) were collected on the piles of magnetite and analyzed for TPH full scan, total RCRA metals, PCBs, VOCs, SVOCs, and gamma spectroscopy. Waste characterization sampling confirmed that $\mathrm{COCs}$ are not present. Based on waste characterization results, the magnetite was disposed of as sanitary waste.

\subsubsection{CAS 03-22-07 Waste Characterization}

Waste generated at this site included two 5-gallon metal buckets containing tar and scattered debris, which consisted of a wood cable spool, wood, rope and scrap metal. On October 11 , 2001, the sample (032207-0-1) was collected from inside the two 5-gallon buckets and analyzed for TPH full scan, total RCRA metals, PCBs, VOCs, SVOCs, and gamma spectroscopy. Results for TPH were higher than the state of Nevada action levels (Nevada Administrative Code [NAC], 2000). Results for SVOCs and VOCs were higher than the Region 9 PRGs for industrial soils, but below 40 CFR 261.24 Table 1 values for the determination of hazardous 
waste (EPA, 1996c). Based on waste characterization results, the two 5 -gallon buckets containing tar was categorized as hydrocarbon waste. The debris that was generated from closure was disposed of as sanitary waste.

\subsubsection{CAS 03-22-10 Waste Characterization}

Waste generated at this site included an empty 55-gallon drum. On October 10, 2001, the soil samples (032210-0-0 and 032210-0-1) were collected underneath the drum from the surface soil and analyzed for TPH, total RCRA metals, PCBs, VOCs, SVOCs, and gamma spectroscopy. The soil beneath the drum was sampled to determine if contents from the now empty drum impacted the soil beneath. Waste characterization sampling confirmed that COCs are not present. The empty 55-gallon drum was disposed of as sanitary waste.

\subsubsection{CAS 03-22-12 Waste Characterization}

Waste generated at this site included a 55 -gallon drum containing a small amount of lubricating grease. On October 11,2001, the soil sample (032212-0-1) was collected from the lubricating grease inside of the drum and analyzed for TPH, total RCRA metals, PCBs, VOCs, SVOCs, and gamma spectroscopy. The results for the lubricating grease sample analyzed for TPH was higher than the state of Nevada action levels (NAC, 2000). Based on waste characterization results, the waste generated from closure was categorized as hydrocarbon waste. The drum was located on a historical mud pit and because drilling mud may already contain TPH, no sample was collected from beneath the drum. The mud pit will be closed as per the Mud Pit Identification Report, Nevada Test Site, Nevada (DOE/NV, 2001).

\subsubsection{CAS 03-22-14 Waste Characterization}

Waste generated at this site included two 5-gallon buckets containing small amounts of tar with some spilled on soil. On October 10, 2001, the soil sample (032214-0-1) was collected from inside of the buckets and analyzed for TPH, total RCRA metals, PCBs, VOCs, SVOCs, and gamma spectroscopy. Results for TPH were higher than the state of Nevada action levels (NAC, 2000), Results for SVOCs were higher than the Region 9 PRGs for industrial soils, but below 40 CFR 264.24 Table 1 values for the determination of hazardous waste (EPA, 1996c). Based on waste characterization results, the waste generated from closure was categorized as hydrocarbon waste.

\subsubsection{CAS 03-22-24 Waste Characterization}

Waste generated at this site included an empty 55-gallon drum. On November 6, 2001, the soil sample (032224-0-1) was collected from the surface soil underneath the drum and analyzed for TPH, total RCRA metals, PCBs, VOCs, SVOCs, and gamma spectroscopy. The soil beneath the drum was sampled to determine if contents from the now empty drum impacted the soil beneath. Waste characterization sampling confirmed that COCs are not present and the drum was disposed of as sanitary waste. 


\subsubsection{CAS 03-22-25 Waste Characterization}

Waste generated at this site included three 5 -gallon containers in which one container was empty and two were full with a white powder material. On October 11, 2001, the sample (032225-0-1) was collected from inside the container was analyzed for total RCRA metals, Inductively Coupled Plasma (ICP) analysis for metals, $\mathrm{pH}$, and gamma spectroscopy. Results for the ICP and $\mathrm{pH}$ showed that the white powder material is sodium hydroxide. Since the sodium hydroxide is already containerized and in a solid form, the material was disposed of as sanitary waste.

\subsubsection{CAS 03-22-26 Waste Characterization}

Waste generated at this site included an empty 5-gallon can. On October 9, 2001, the samples (032226-0-0 and 032226-0-1) were collected from the surface soil underneath the can and analyzed for TPH, total RCRA metals, PCBs, VOCs, SVOCs, and gamma spectroscopy. The soil beneath the can was sampled to determine if contents from the now empty can impacted the soil beneath. Waste characterization sampling confirmed that COCs are not present. The can was disposed of as sanitary waste.

\subsubsection{CAS 03-22-30 Waste Characterization}

Waste generated at this site included a large pile of debris, which contained empty nonpressurized aerosol cans, wood, scrap metal, cable, wire, and electrical conduit. No waste characterization sampling was done, due to process knowledge that the debris is discarded construction material and does not contain COCs. The debris was free to be released as sanitary waste.

\subsubsection{CAS 03-22-34 Waste Characterization}

Waste generated at this site included an empty 15-gallon drum. On October 9, 2001, the sample (032234-0-1) was collected from surface soil underneath the drum and analyzed for TPH, total RCRA metals, PCBs, VOCs, SVOCs, and gamma spectroscopy. The soil beneath the drum was sampled to determine if contents from the now empty drum impacted the soil beneath. Waste characterization sampling confirmed that COCs are not present and the waste material was free to be released as sanitary waste.

\subsubsection{CAS 03-22-37 Waste Characterization}

Waste generated at this site included a 5 -gallon bucket that was partially buried in soil. On October 7, 2001, the soil sample (032237-0-1) was collected from underneath the bucket and analyzed for TPH, total RCRA metals, PCBs, VOCs, SVOCs, and gamma spectroscopy. The soil sample was a composite of soil inside the bucket and beneath it. Based on waste characterization results, the waste material was free to be released as sanitary waste and the soil does not contain COCs. 


\subsubsection{CAS 03-24-08 Waste Characterization}

Waste generated at this site included a vehicle battery that was intact and appeared to be in good condition. On October 10, 2001, the soil sample (032408-0-1) was collected directly underneath the vehicle battery from the surface soil and analyzed for TCLP metals-lead and soil pH. Based on waste characterization results, the soils were free of COCs. The vehicle battery was released to Fleet Operations to be recycled.

\subsubsection{CAS 03-99-10 Waste Characterization}

Waste generated at this site included an empty 5-gallon gasoline can. On October 10, 2001, the sample (039910-0-1) was collected from the surface soil underneath the gasoline can and analyzed for TCLP metals-lead and TPH-gasoline range. The soil beneath the can was sampled to determine if contents from the now empty can impacted the soil beneath. Based on waste characterization results, the gasoline can was free to be released as sanitary waste.

\subsection{SITE CLOSURE ACTIVITIES}

\subsubsection{CAS 01-24-03: Batteries (2)}

A small pile of 50-caliber shells and soil containing broken battery pieces were removed from the site on November 29, 2001. The shells were placed into plastic bags and transported to the Area 9 U10c Landfill for disposal. The soil containing broken battery pieces was characterized as being hazardous waste (lead) and placed into a 15-gallon drum staged in a Satellite Accumulation Area (\#NTS0105). The lead impacted soil was removed using shovels. A handheld water sprayer was used to reduce a lead dust hazard during the soil removal activities. Waste Management will have the drum transported off-site for disposal. A field screening method (X-ray Fluorescence Device [XRF]) was used to determine how much soil needed to be removed. Verification soil sample $(012403-0-V)$ was collected from the excavation created by removing the broken battery pieces. The sample was taken at approximately 3 inches in depth and analyzed for TCLP metals-lead. Results showed TCLP metals-lead lower than the EPA Region 9 PRGs for Industrial Soil (EPA, 1996a). Analytical results for the verification sample are given in Table 2. All closure activities were documented with photographs and field notes. No further actions are required at this site.

\subsubsection{CAS 03-14-05: Transformers; Switch Boxes}

Two transformers (non-PCB) were removed from the vault on December 12, 2001, and two associated switches were drained, one in the vault and the other outside the vault. The switch located outside the vault was left in place because it was still active in Area 3 . The oils were drained on November 30,2001, and transported to Site Services Department to be recycled. The two transformers were transported to the Area $9 \mathrm{U} 10 \mathrm{c}$ Landfill for disposal. Clean closure of this site was verified by visual inspection. All closure activities were documented with photographs and field notes. No further action is required this site. 


\subsubsection{CAS 03-22-06: Drums; Bucket; Can}

The Preliminary Assessment Database documents state that the drum, bucket, and can have already been removed. Several past BN/ER site visits concur that the debris material has been removed. The two small piles of magnetite also associated with this site were removed on November 29, 2001. The magnetite was placed into an end-dump using a front-end loader and taken to the Area 9 U10c Landfill for disposal. All closure activities were documented with photographs and field notes. Clean closure of this site was verified by visual inspection; no verification samples were required or collected. No further action is required at this site.

\subsubsection{CAS 03-22-07: Buckets (2)}

The two 5-gallon buckets containing tar and scattered debris were removed on November 27, 2001. The two 5-gallon buckets were removed by hand and transported to the Area 6 Hydrocarbon Landfill for disposal. A verification soil sample (032207-0-V) was collected from the former location of the buckets at approximately 2 inches in depth and analyzed for TPH, SVOCs, and VOCs. Results showed TPH lower than the state of Nevada action level (NAC, 2000). Results showed SVOCs and VOCs lower than the Region 9 PRGs for industrial soils (EPA, 1996a). Analytical results for the verification samples are given in Tables 3 and 4 . The scattered debris, which included a wood cable spool, wood, rope, and scrap metal were also removed at the same time and transported to the Area 9 U10c Landfill for disposal. All closure activities were documented with photographs and field notes. No further actions are required at this site.

\subsubsection{CAS 03-22-10: Drum}

The empty 55-gallon drum was removed by hand on November 27, 2001, and transported to the Area 9 U10c Landfill for disposal. Clean closure of this site was verified by visual inspection; no verification samples were required or collected. All closure activities were documented with photographs and field notes. No further action is required at this site.

\subsubsection{CAS 03-22-12: Drum}

The 55-gallon drum containing small amounts of lubricating grease was removed by hand on November 27, 2001, and transported to the Area 6 Hydrocarbon Landfill for disposal. Clean closure of this site was verified by visual inspection. Verification samples were not collected because the lubricating grease was contained inside of the drum and the drum was located inside a mud pit. It would not be possible to differentiate mud pit COCs from a spill from the drum. The mud pit will be closed as per the Mud Pit Identification Report, Nevada Test Site, Nevada (DOE/NV, 2001). All closure activities were documented with photographs and field notes. No further action is required at this site. 
TABLE 2-SUMMARY OF ANALYTICAL RESULTS FOR TCLP METALSLEAD AND pH IN VERIFICATION SOIL SAMPLES

\begin{tabular}{|c|c|c|c|c|c|c|c|c|}
\hline $\begin{array}{c}\text { SAMPLE } \\
\text { IDENTIFICATION }\end{array}$ & $\begin{array}{l}.0 \\
\frac{1}{8} \\
\frac{2}{5}\end{array}$ & $\stackrel{\Xi}{\Xi}$ & 害 & 音 & $\stackrel{\mathscr{Z}}{\mathbb{S}}$ & 疍 & 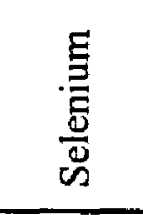 & $\stackrel{5}{\overline{2}}$ \\
\hline $\begin{array}{l}\text { EPA Industrial PRG } \\
(\mathrm{mg} / \mathrm{kg})^{\mathrm{b}}\end{array}$ & 440 & 100,000 & 810 & 450 & 750 & 610 & 10,000 & 10,000 \\
\hline \multicolumn{9}{|l|}{ CAS 01-24-03 } \\
\hline $012403-0-V$ & $N / A^{c}$ & $N / A$ & N/A & N/A & $\mathrm{ND}^{\mathrm{d}}$ & N/A & N/A & N/A \\
\hline
\end{tabular}

Note: All samples analyzed by U.S. Environmental Protection Agency (EPA) Method SW-846 6010 (EPA, 1996b).

See analytical reports in Appendix A for detection limits.

${ }^{3} E P A$ Region 9 Industrial Preliminary Remediation Goasl (PRGs) for 1996 (EPA, 1996b).

${ }^{\mathrm{b}} \mathrm{mg} / \mathrm{kg}$-milligram per kilogram.

canalysis did not apply to this sample.

ND=not detected at the reporting limit.

\begin{tabular}{||c|c|c||}
\hline SAMPLE IDENTIFICATION & $\begin{array}{c}\text { pH } \\
\text { (white powder material } \\
\text { inside containers) }\end{array}$ & $\begin{array}{c}\text { pH } \\
\text { (soil underneath containers } \\
\text { after cleanup completed) }\end{array}$ \\
\hline CAS 03-22-25 & & \\
\hline $032225-0-\mathrm{V}$ & 13.4 & 9.62 \\
\hline
\end{tabular}



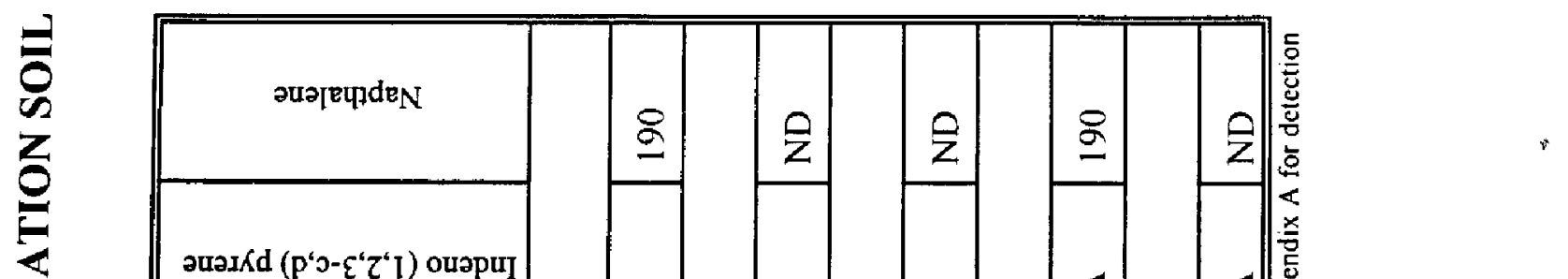

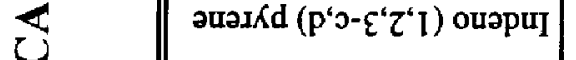
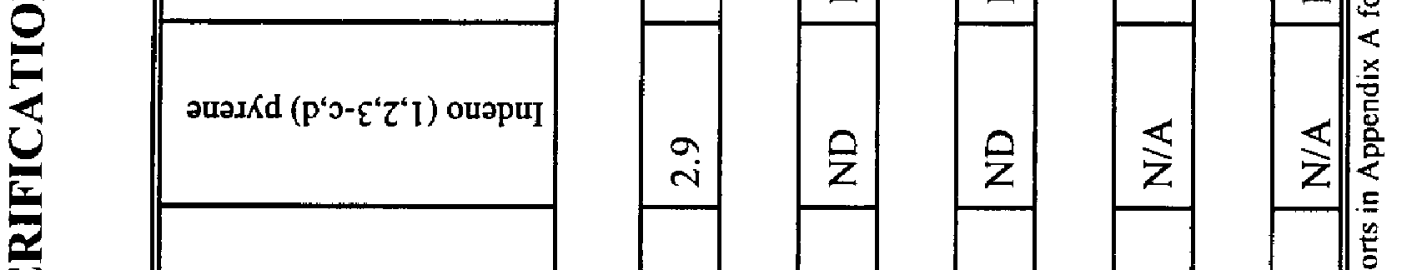

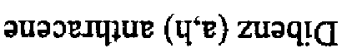
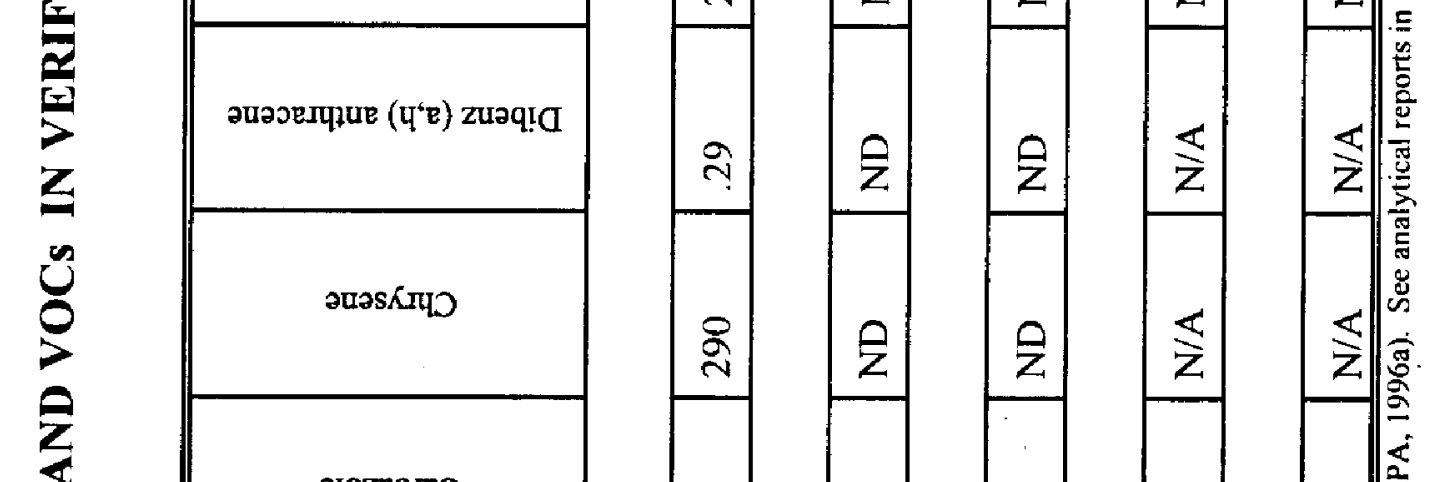

\section{.} .

.
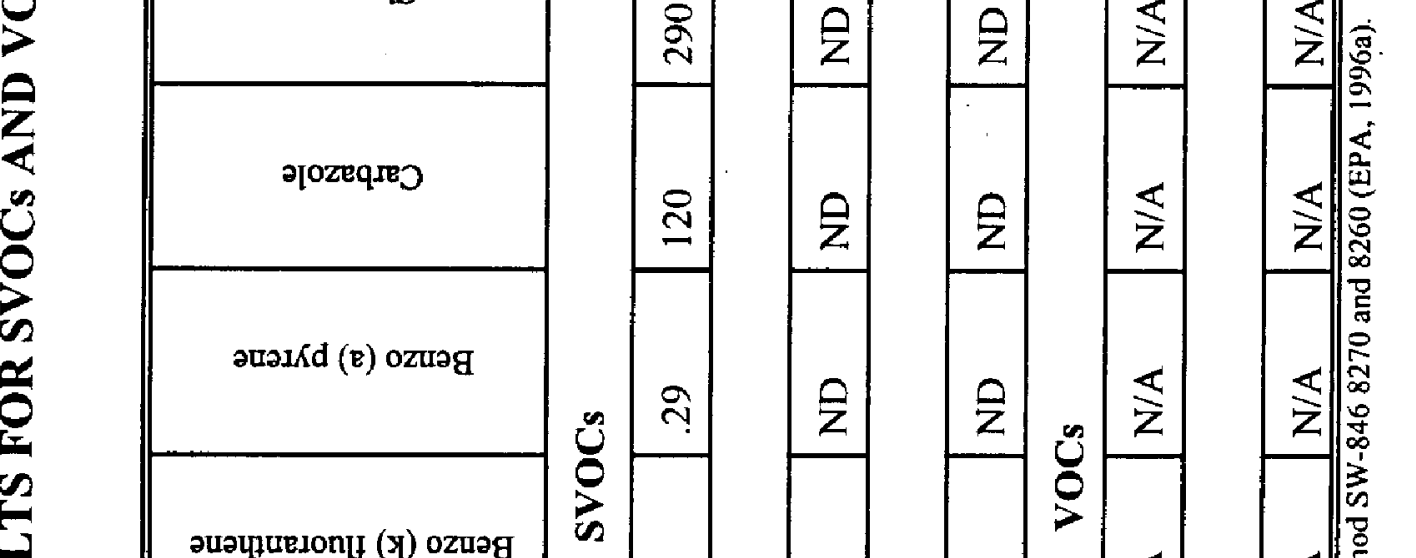

宓

द

aนas KIำ

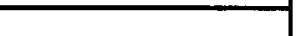

गовеqле

5

$\sum_{2}^{3}$

Мәэедчрие (e) оzuәg

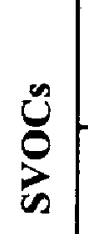

ते

욜

2

z

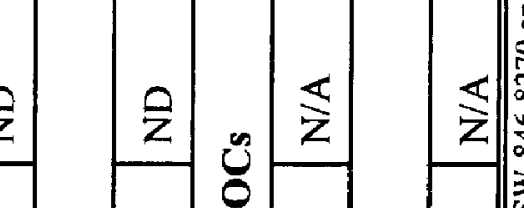

离 
TABLE 4-SUMMARY OF ANALYTICAL RESULTS FOR TOTAL PETROLEUM HYDROCARBONS IN VERIFICATION SOIL SAMPLES

\begin{tabular}{|c|c|c|c|c|}
\hline SAMPLE IDENTIFICATION & $\begin{array}{l}\text { GASOLINE } \\
\text { RANGE } \\
(\mathrm{mg} / \mathrm{kg})\end{array}$ & $\begin{array}{l}\text { DIESEL } \\
\text { RANGE } \\
\text { (mg/kg) }\end{array}$ & $\begin{array}{l}\text { OIL } \\
\text { RANGE } \\
\text { (mg/kg) }\end{array}$ & $\begin{array}{c}\text { TOTAL PETROLEUM } \\
\text { HYDROCARBONS } \\
\text { (mg/kg) }\end{array}$ \\
\hline $\begin{array}{l}\text { NDEP }{ }^{\mathrm{a}} \text { Action Level } \\
(\mathrm{mg} / \mathrm{kg})^{\mathrm{b}}\end{array}$ & 100 & 100 & 100 & 100 \\
\hline \multicolumn{5}{|l|}{ CAS 03-22-07 } \\
\hline $032207-0-\mathrm{V}$ & $\mathrm{ND}^{\mathrm{c}}$ & $\mathrm{ND}$ & $\mathrm{ND}$ & $\mathrm{ND}$ \\
\hline \multicolumn{5}{|l|}{ CAS 03-22-14 } \\
\hline $032214-0-\mathrm{V}$ & ND & $\mathrm{ND}$ & ND & $\mathrm{ND}$ \\
\hline
\end{tabular}

${ }^{2}$ NDEP-Nevada Division of Environmental Protection.

${ }^{\mathrm{b}} \mathrm{mg} / \mathrm{kg}=$ =milligrams per kilogram.

$\mathrm{ND}=$ not detected for the laboratory reporting limits. 


\subsubsection{CAS 03-22-14: Drums (2)}

The Preliminary Assessment Database describes the site as containing two drums, however after several $\mathrm{BN} / \mathrm{ER}$ site visits, it was determined that the drums were actually two 5-gallon buckets containing a small amount of spilled tar. The buckets and tar were removed using shovels on November 27, 2001, and transported to the Area 6 Hydrocarbon Landfill for disposal. Verification soil sample (032214-0-V) was collected from the former location of the buckets at approximately 3 inches in depth and analyzed for TPH and SVOCs. Results showed TPH lower than the state of Nevada action level and SVOCs lower than the Region 9 PRGs for industrial soils (NAC, 2000; EPA, 1996a), verifying that the site was clean-closed. Analytical results for the verification samples are given in Tables 3 and 4 . All closure activities were documented with photographs and field notes. No further actions are required at this site.

\subsubsection{CAS 03-22-24: Drum}

The empty 55-gallon drum was removed by hand on November 27, 2001, and transported to the Area 9 U10c Landfill for disposal. Clean closure of this site was verified by visual inspection; no verification samples were required or collected. All closure activities were documented with photographs and field notes. No further action is required at this site.

\subsubsection{CAS 03-22-25: Drum}

The Preliminary Assessment Database describes the site as containing a drum, however after several BN/ER site visits, it was determined that the site contained three 5-gallon containers. Two containers were full of sodium hydroxide (solid), while the third container was empty. This was determined from waste characterization sampling results. The three 5 -gallon containers were removed on January 25, 2002, and transported to the Area 9 U10c Landfill for disposal. Verification soil sample $(032225-0-\mathrm{V})$ was collected from the surface soil at the former location of the containers and analyzed for soil $\mathrm{pH}$. Results showed that $\mathrm{pH}$ in the soil underneath the containers was at an acceptable range for desert soils to show that the site was clean closed. Analytical results for verification samples are given in Table 2. All closure activities were documented with photographs and field notes. No further actions are required at this site.

\subsubsection{CAS 03-22-26: Can}

The empty 5-gallon can was removed by hand on November 27,2001 , and transported to the Area 9 U10c Landfill for disposal. Clean closure of this site was verified by visual inspection; no verification samples were required or collected. All closure activities were documented with photographs and field notes. No further action is required at this site. 


\subsubsection{CAS 03-22-30: Buckets; Cans; Debris}

The large pile of debris, which included empty non-pressurized aerosol cans, wood debris, scrap metal, cable, wire and electrical conduit, were removed on November 29, 2001, by placing debris into an end dump using a front-end loader and transporting it to the Area 9 U10c Landfill for disposal. Clean closure of this site was verified by visual inspection; no verification samples were required or collected. All closure activities were documented with photographs and field notes. No further action is required at this site.

\subsubsection{CAS 03-22-34: Drum}

The empty 15-gallon drum was removed by hand on November 27, 2001, and transported to the Area 9 U10c Landfill for disposal. Clean closure of this site was verified by visual inspection; no verification samples were required or collected. All closure activities were documented with photographs and field notes. No further action is required at this site.

\subsubsection{CAS 03-22-37: Drum}

The Preliminary Assessment Database describes the site as containing a drum, however after several BN/ER site visits, it was determined that the site contained a partially buried, 5-gallon bucket. The 5-gallon bucket was removed by hand on December 5, 2001, and transported to the Area $9 \mathrm{U10c}$ Landfill for disposal. Waste characterization sample results verified that no COCs were present underneath or inside

the bucket. Clean closure of this site was verified by visual inspection; no verification samples were required or collected. All closure activities were documented with photographs and field notes. No further action is required at this site.

\subsubsection{CAS 03-24-08: Battery}

The vehicle battery was removed by hand on November 27, 2001, and transported to Fleet Operations to be recycled. Clean closure of this site was verified by visual inspection; no verification samples were required or collected. All closure activities were documented with photographs and field notes. No further action is required at this site.

\subsubsection{CAS 03-99-10: Gas Can}

The empty 5-gallon gasoline can was removed by hand on November 27, 2001, and transported to the Area 9 U10c Landfill for disposal. Clean closure of this site was verified by visual inspection; no verification samples were required or collected. All closure activities were documented with photographs and field notes. No further action is required at this site. 
CLOSURE REPORT - CAU 343 Areas 1.3.\& 4 Housekeeping Sites Section: Closure Activities Revision 0 Date rebruary 2002

\section{THIS PAGE INTENTIONALLY LEFT BLANK}




\subsection{WASTE DISPOSITION}

Wastes generated during the closure of CAU 343: Areas 1, 3, \& 4 Housekeeping Sites were disposed as follows:

- CAS 01-24-03: A 15-gallon drum was filled with lead-impacted soil. The drum containing soil is now staged at the Hazardous Waste Storage Pad in Area 5 and will be shipped off-site for disposal within one year. In addition, approximately 30 to 4050 caliber brass shells were disposed of in the Area 9 U10c Landfill

- CAS 03-14-05: Two transformers (non-PCB) were disposed of as sanitary waste in the Area 9 U10c Landfill. Drained oils from two switches were recycled by Site Services Department.

- CAS 03-22-06: Approximately 10.8 cubic meters (14 cubic yards) of magnetite and associated soil disposed of as sanitary waste in the Area 9 U10c Landfill.

- CAS 03-22-07: Two 5-gallon buckets containing tar and associated soils were disposed of in the Area 6 Hydrocarbon Landfill, while debris disposed of as sanitary waste was disposed of in the Area 9 U10c Landfill.

- CAS 03-22-10: An empty 55-gallon drum was disposed of as sanitary waste in the Area 9 U10c Landfill.

- CAS 03-22-12: A 55-gallon drum containing a small amount of lubricating grease inside of the drum was disposed of in the Area 6 Hydrocarbon Landfill.

- CAS 03-22-14: Two 5-gallon buckets containing tar and small amounts of spilled tar/associated soils were disposed of in the Area 6 Hydrocarbon Landfill.

- CAS 03-22-24: An empty 55-gallon drum was disposed of as sanitary waste in the Area 9 U10c Landfill.

- CAS 03-22-25: Three 5-gallon containers, two containing sodium hydroxide (solid) and the third was empty. All containers were disposed of as sanitary waste in the Area 9 U10c Landfill.

- CAS 03-22-26: An empty 5-gallon can was disposed of as sanitary waste in the Area 9 U10c Landfill.

- CAS 03-22-30: A large pile of debris was disposed of as sanitary waste in the Area 9 U10c Landfill. 
- CAS 03-22-34: An empty 15-gallon drum was disposed of as sanitary waste in the Area 9 U10c Landfill.

- CAS 03-22-37: A partially buried 5-gallon bucket was disposed of as sanitary waste in the Area 9 U10c Landfill.

- CAS 03-24-08: A vehicle battery was transported to Fleet Operations to be recycled.

- CAS 03-99-10: An empty 5-gallon gasoline can was disposed of as sanitary waste in the Area 9 U10c Landfill. 


\subsection{CLOSURE VERIFICATION}

The following four CASs required verification soil sampling:

- $01-24-03$

- 03-22-07

- $03-22-14$

- $03-22-25$

Samples were collected from a total of four CASs after the removal of debris and small amounts of soil as determined by field screening and/or by visible inspection. The samples were collected with clean disposable plastic scoops and placed in labeled sample containers secured with custody seals. The sample containers were placed on ice in a cooler; transported under chain of custody to the BN Sample Management group in Mercury, Nevada; and shipped to an off-site laboratory for analysis. The four CASs requiring verification sampling were analyzed for the following:

- CAS 01-24-03: TCLP metals-lead

- CAS 03-22-07: TPH, SVOCs, and VOCs

- CAS 03-22-14: TPH and SVOCs

- CAS 03-22-25: soil pH

The analytical results verify that the levels of SVOCs, VOCs, TCLP metals-lead, and TPH remaining in the ground at three CASs are non-detectable or below EPA Region 9 PRGs for industrial soil (EPA, 1996a), and the state of Nevada action level for TPH (i.e., $100 \mathrm{mg} / \mathrm{kg}$ ) (NAC, 2000). At CAS 03-22-25 soil pH was normal for NTS soils. The analytical results for SVOCs, VOCs, TCLP metals-lead, $\mathrm{pH}$, and TPH are summarized in Tables 2, 3, and 4, respectively; the analytical reports are included in Appendix A. 
CLOSURE REPORT - CAU 343 Areas 1, 3, \& 4 Housekeeping Sites

Section: Closure Veritication Revision: 0 Date: February 2002

THIS PAGE INTENTIONALLY LEFT BLANK 


\subsection{SUMMARY AND RECOMMENDATIONS}

\subsection{SUMMARY}

The following site closure activities were performed at the 15 of the 18 CASs comprising

CAU 343 and are documented in the report:

- All debris (e.g., wood, metal cable, scrap metal, rope, electrical debris, wire, empty nonpressurized aerosol cans, rubber, 50-caliber brass shells, and dry transformers) were removed from the sites and disposed of in the Area 9 U10c Landfill.

- All containers (e.g., drums, cans, buckets, and containers) were disposed of in either the Area 9 U10c Landfill or the Area 6 Hydrocarbon Landfill, depending on its contents.

- An intact vehicle battery was removed from one site and transported to Fleet Operations to be recycled. Another site contained broken pieces of a battery in soil and was determined to be hazardous waste (lead). This waste is now being staged at the Hazardous Waste Storage Pad in Area 5 to eventually be shipped off-site for disposal.

- All soil containing TPH levels at or above the state of Nevada TPH action level (i.e., $100 \mathrm{mg} / \mathrm{kg}$ ) (NAC, 2000) was removed and disposed of in the NTS Area 6 Hydrocarbon Landfill. These CASs include, CAS 03-22-07 and CAS 03-22-14.

- All material, including soil, containing magnetite was removed from one site and disposed of in the Area 9 U10c Landfill.

\subsection{RECOMMENDATIONS}

Since the closure activities for CAU 343 have been completed following the NDEP-approved Sectored Clean-up Work Plan for Housekeeping Category Waste Sites (DOE/NV, 2000) as documented in this report, the U.S. Department of Energy, National Nuclear Security Administration Nevada Operations Office (NNSA/NV) requests the following:

- A Notice of Completion be provided by the NDEP to the NNSA/NV for the closure of CAU 343 (CAS 01-22-06, CAS 01-24-03, CAS 03-14-05, CAS 03-22-06, CAS 03-22-07, CAS 03-22-10, CAS 03-22-12, CAS 03-22-14, CAS 03-22-24, CAS 03-22-25, CAS 03-22-26, CAS 03-22-30, CAS 03-22-34, CAS 03-22-37, CAS 03-24-08 and CAS 03-99-10).

- CAU 343 be moved from Appendix III to Appendix IV of the FFACO Closed Corrective Action Units (FFACO, 1996). 
CLOSURE REPORT - CAU 343

Areas 1, 3, \& 4 Housekeeping Sites

Section: Summary and Recommendations Revision: 0

Date: February 2002

\section{THIS PAGE INTENTIONALLY LEFT BLANK}




\subsection{REFERENCES}

BN, see Bechtel Nevada.

Bechtel Nevada, 2000. Generic Field Management Plan for Housekeeping Category Waste Sites, Las Vegas, NV.

Bechtel Nevada. 2001. Site-Specific Health and Safety Plan for Corrective Action Unit 343: Areas 1, 3, \& 4 Housekeeping Sites, Nevada Test Site, Nevada, Las Vegas, NV.

DOE/NV, see U.S. Department of Energy, Nevada Operations Office.

EPA, see U.S. Environmental Protection Agency.

FFACO, see Federal Facility Agreement and Consent Order.

Federal Facility Agreement and Consent Order of 1996. Agreed to by the Nevada Division of Environmental Protection, U.S. Department of Energy, and U.S. Department of Defense.

NAC, see Nevada Administrative Code.

Nevada Administrative Code. 2000. NAC 445A.2272, Contamination of soil: Establishment of action levels. As adopted by the Nevada Environmental Commission, October, Carson City, NV.

U.S. Department of Energy, Nevada Operations Office. 2000. Sectored Clean-up Work Plan for Housekeeping Category Waste Sites, Rev. 0, DOE/NV--579, Las Vegas, NV.

U.S. Department of Energy, Nevada Operations Office. September 2001. Mud Pit Identification Report, Nevada Test Site, Nevada, DOE/NV--752, Las Vegas, NV.

U.S. Environmental Protection Agency. 1996a. Region IX Preliminary Remediation Goals (PRGS), San Francisco, CA.

U.S. Environmental Protection Agency. 1996b. Test Methods for Evaluating Solid Waste, Physical/Chemical Methods, EPA Publication SW-846, Third Edition. Washington, D.C.

U.S. Environmental Protection Agency. 1996c. Title 40 Code of Federal Regulations 261.24, Toxicity Characteristic, Washington, D.C. 
CLOSURE REPORT - CAU 343

Areas l, 3, \& 4 Housekeeping Sites

Section: References

Revision: 0

Date: February 2002

THIS PAGE INTENTIONALLY LEFT BLANK 


\section{APPENDIX A VERIFICATION SAMPLE ANALYTICAL RESULTS}


THIS PAGE INTENTIONALLY LEFT BLANK 


\section{APPENDIX A TABLE OF CONTENTS}

Analytical Results for Sample $012403-0-V \ldots \ldots \ldots \ldots \ldots \ldots \ldots \ldots \ldots$ A $\ldots \ldots \ldots$ to A-5

Analytical Results for Sample $032225-0-V \ldots \ldots \ldots \ldots \ldots \ldots \ldots \ldots \ldots$. . . . . . . . . . . . A-9

Analytical Results for Samples CAU343-TB9, CAU343-TB10, 032207-0-V, and

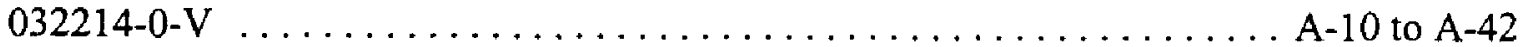


THIS PAGE INTENTIONALLY LEFT BLANK 
CLIENT: $\quad$ Bechtel Nevada

P.O. Box 98521 , M/S NTS273

Las Vegas, NV $89193-8521$

ATTN: $\quad$ Ted Redding

PROJECT NAME: V1366

NEL ORDER ID: L0112008

PROJECT NUMBER: 30033

Attached are the analytical results for samples in support of the above referenced project.

Samples submitted for this project were not sampled by NEL Laboratories. Samples were received by NEL in good condition, under chain of custody on 12/3/01.

Should you have any questions or comments, please feel free to contact our Client Services department at (702) 657-1010.
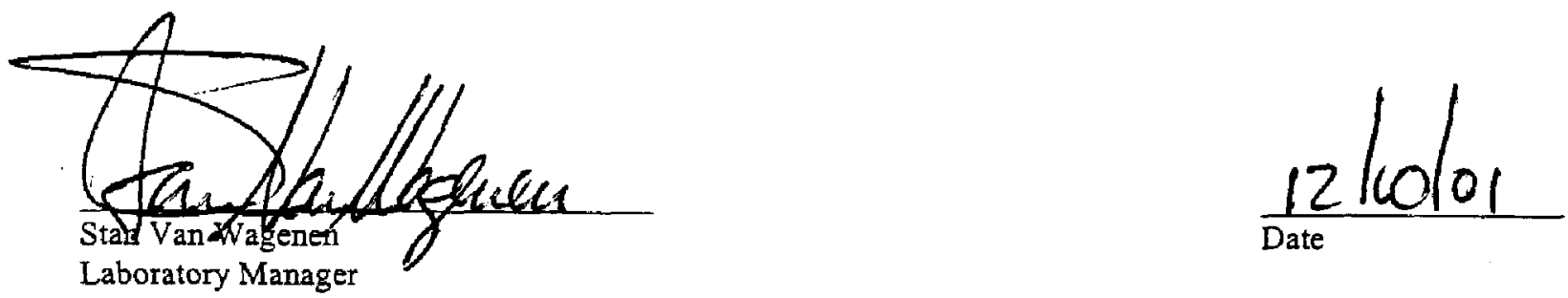

\section{CERTIFICATIONS:}

\begin{tabular}{|c|c|c|c|c|c|c|c|}
\hline & Reno & Las Vegas & S. California & & Reno & Las Vegas & S. California \\
\hline Arizona & AZ0520 & AZ0518 & AZ0605 & Idaho & $\overline{\text { Certified }}$ & Certified & \\
\hline Cali & 1707 & 2002 & 2264 & Montana & Certified & Certified & \\
\hline $\begin{array}{l}\text { US Army Corps } \\
\text { of Engineers }\end{array}$ & Certified & Certified & & $\begin{array}{l}\text { Nevada } \\
\text { L.A.C.S.D. }\end{array}$ & NV033 & NV052 & $\begin{array}{l}\text { CA084 } \\
10228\end{array}$ \\
\hline
\end{tabular}


NEL LABORATORIES

CLIENT: Bechtel Nevada

PROJECT ID: V1366

PROJECT \#: 30033

TEST:

MATRIX:
CLIENT D:

TIED: $11 / 29 / 0$

NEL SAMPLE ID: L0112008-01

\begin{tabular}{|c|c|c|c|c|c|c|}
\hline PARAMETER & $\begin{array}{c}\text { RESULT } \\
\mathrm{mg} / \mathrm{L} \\
\end{array}$ & $\begin{array}{c}\text { REPORTING } \\
\text { LIMUT } \\
\end{array}$ & D. F. METHOD & $\begin{array}{c}\text { TCLP/STLC } \\
\text { EXTRACTION } \\
\text { DATE } \\
\end{array}$ & DIGESTED & ANALYZED \\
\hline Lead & ND & $0.05 \mathrm{mg} / \mathrm{L}$ & EPA 6010 & $12 / 4 / 01$ & $12 / 5 / 01$ & $12 / 6 / 01$ \\
\hline
\end{tabular}

J.F. - Dilution Factor

JD - Not Detected

This report shall not be reproduced except in full, without the written approval of the laboratory. 


\section{NEL LABORATORIES}

\begin{tabular}{llll}
\hline CLIENT: & Bechtel Nevada & CLIENT D: & Methud Blank \\
PROJECT ID: & V1366 & DATE SAMPLED: & NA \\
PROJECT \#: & 30033 & NEL SAMPLE ID: & $1205-1.1$ PB-BLK
\end{tabular}

TEST: TCLP Metais

MATRIX: TCLP Extract

\begin{tabular}{|c|c|c|c|c|c|c|c|}
\hline \multirow[b]{2}{*}{ PARAMETER } & \multirow[b]{2}{*}{ RESULT } & \multirow{2}{*}{$\begin{array}{c}\text { REPORTING } \\
\text { LMIT } \\
\end{array}$} & \multicolumn{5}{|c|}{$\begin{array}{l}\text { TCLP/STLC } \\
\text { EXTRACTION }\end{array}$} \\
\hline & & & D. F. & METHOD & DATE & DIGESTED & ANALYZED \\
\hline cead & ND & $0.05 \mathrm{mg} / \mathrm{L}$ & 1 & EPA 6010 & $12 / 4 / 01$ & $12 / 5 / 01$ & $12 / 6 / 01$ \\
\hline
\end{tabular}

D.F. - Dilution Factor

VD - Not Detected

his report shall not be reproduced except in full, without the written approval of the laboratory. 
NEL LABORATORIES

\begin{tabular}{ll}
\hline CLIENT: & Bechtel Nevada \\
PROJECT ID: & V1366 \\
PROJECT \#: & 30033 \\
TEST: & TCLP/STLC Metals \\
MATRIX: & Solid
\end{tabular}

\section{PARAMETER}

Lead

Lead

Lead
NEL Sample W

1205-1.1PB-LCS

P0111052-06-MS

P0111052-06-MSD

\begin{tabular}{|c|c|c|c|c|}
\hline Spike & Spike & Percent & Acceptable & \\
\hline Amount & Result & Recovery & Range & $\underline{\mathbf{R P D}}$ \\
\hline 1 & 0.928 & 93 & $85-115$ & \\
\hline 1 & 0.858 & 86 & $75-125$ & \\
\hline 1 & 0.868 & 87 & $75-125$ & 1.2 \\
\hline
\end{tabular}

ND - Not Detected

This report shall not be reproduced except in full, without the written approval of the laboratory. 


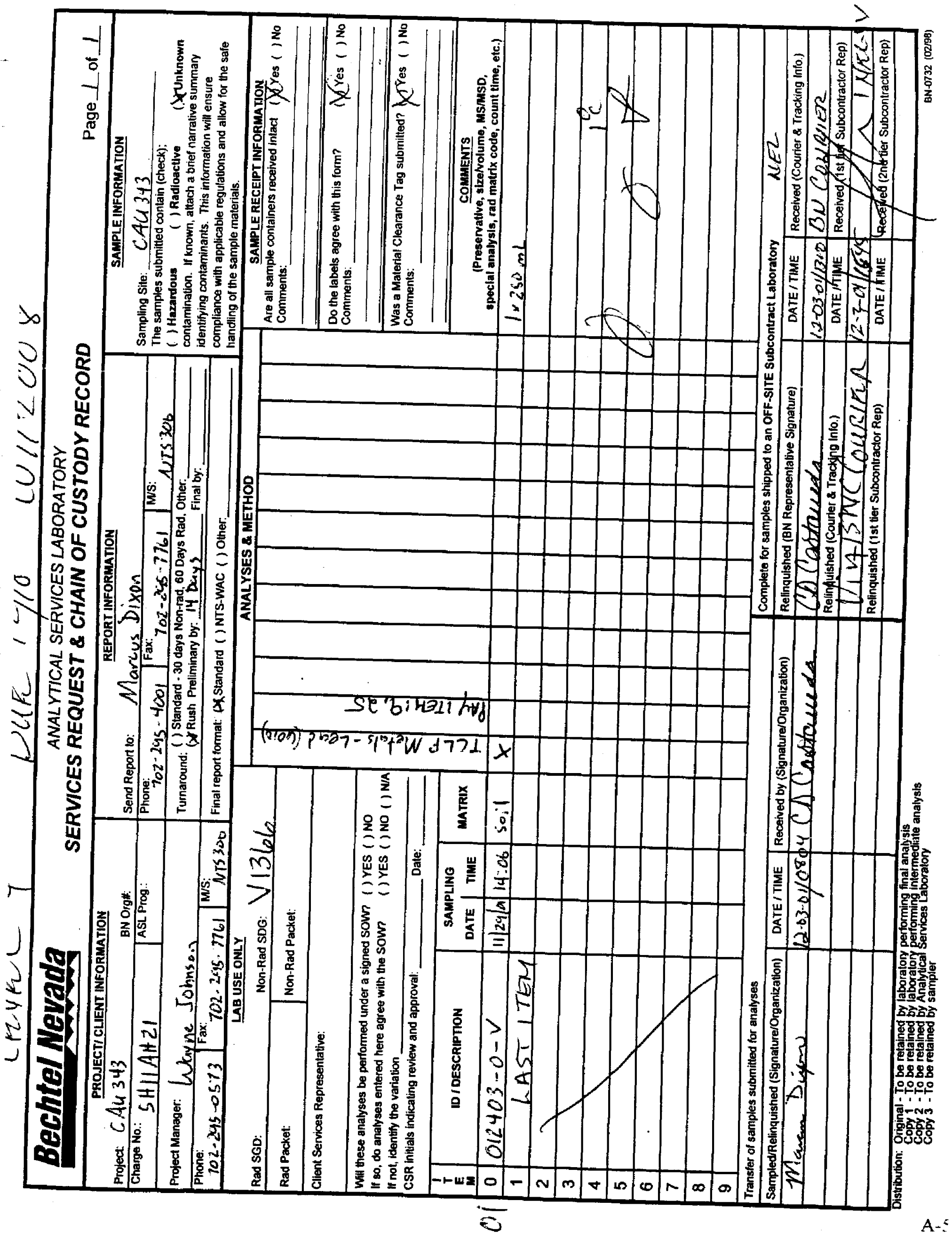


CLIENT:

Bechtel Nevada

P.O. Box 98521, M/S NTS273

Las Vegas, NV $89193-8521$

ATTN:

Ted Redding

PROJECT NAME: $\quad$ V 1370

PROJECT NUMBER: 30033

Attached are the analytical results for samples in support of the above referenced project.

Samples submitted for this project were not sampled by NEL Laboratories. Samples were received by NEL in good condition, under chain of custody on 12/4/01.

Should you have any questions or comments, please feel free to contact our Client Services department at (702) $657-1010$.

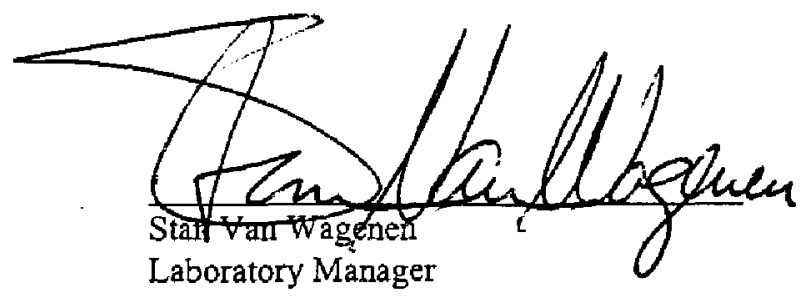

CERTIFICATIONS:

\begin{tabular}{llll}
\hline & Reno & Las Vegas & S. California \\
\cline { 2 - 4 } Arizona & AZ0520 & AZ0518 & AZ0605 \\
California & 1707 & 2002 & 2264 \\
US Army Corps & Certified & Certified & \\
of Engineers & & &
\end{tabular}

NEL ORDER ID: L0112019
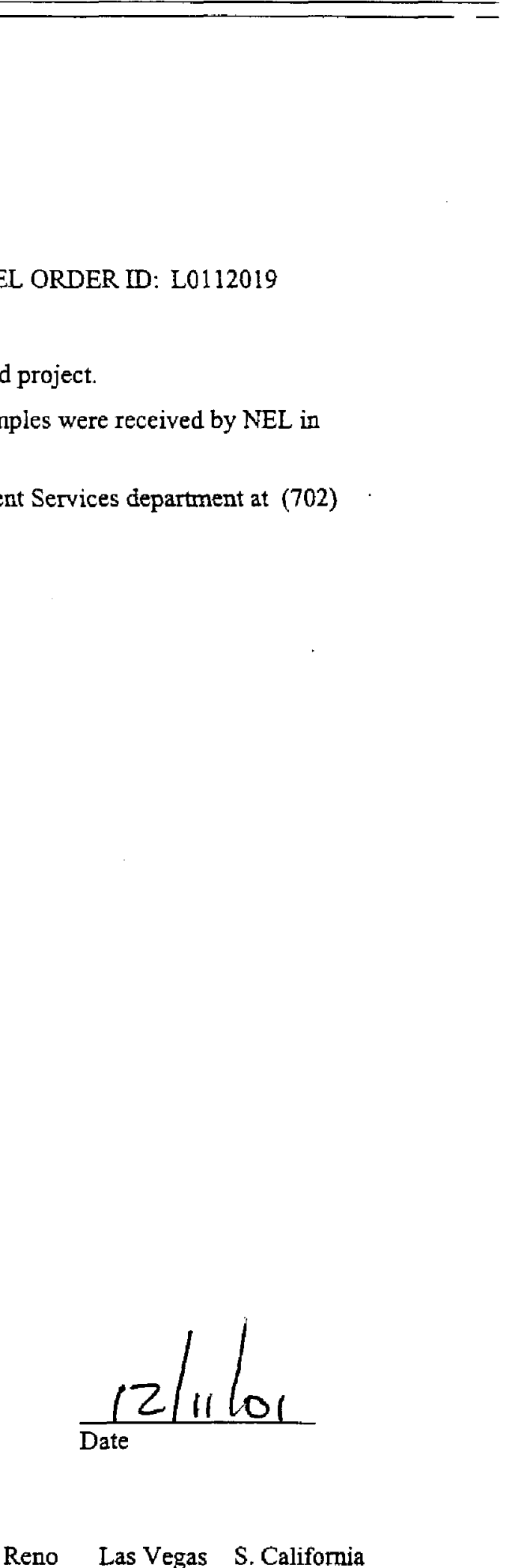

Reno Las Vegas S. Califormia

Idaho

Montana Certified Certified Certified Certified

Nevada

NV033

NV052

CA084

L.A.C.S.D. 
NEL LABORATORIES

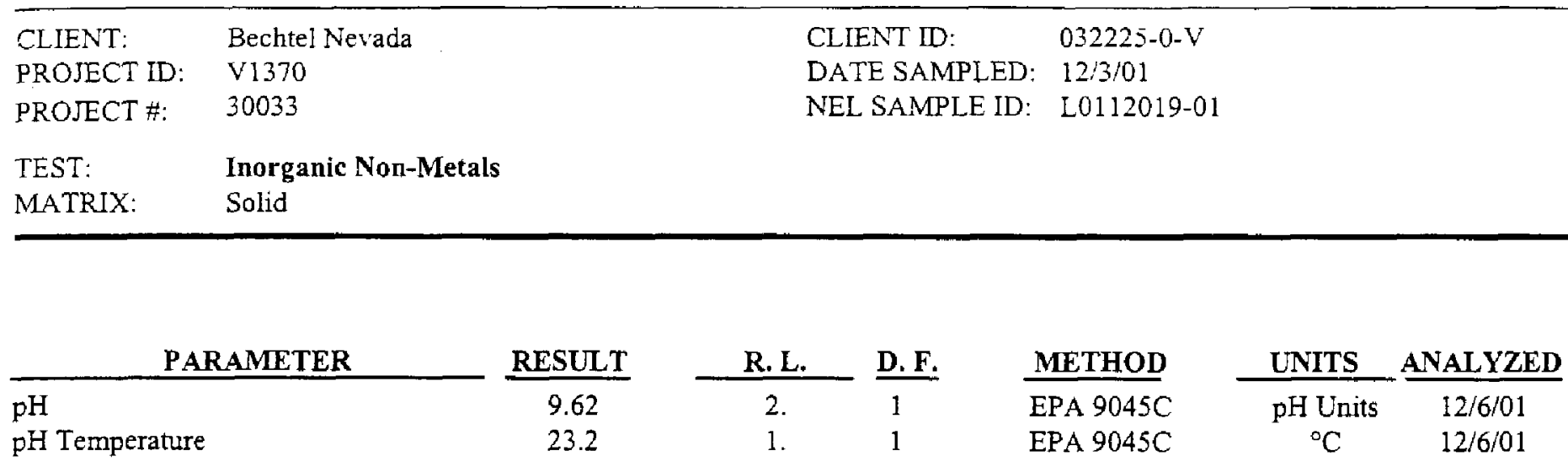

23.2

1

EPA $9045 C$

2.L. - Reporting Limit

J.F. - Dilution Factor

VD - Not Detected

This report shall not be reproduced except in full, without the written approval of the laboratory. 


\begin{tabular}{ll} 
& NEL LABORATORIES \\
\hline CLIENT: & Bechtel Nevada \\
PROJECT ID: & V1370 \\
PROJECT \#: & 30033 \\
TEST: & Inorganic Non-Metals \\
MATRIX: & Solid
\end{tabular}

\begin{tabular}{|c|c|c|c|c|c|c|}
\hline & & Spike & Spike & Percent & Acceptable & \\
\hline PARAMETER & NEL Sample ID & Amount & $\overline{\text { Result }}$ & Recovery & Range & $\underline{\text { RPD }}$ \\
\hline 7.00 Buffer & 011206PHS-LCS & 7 & 7.03 & 100 & $99-101$ & \\
\hline
\end{tabular}




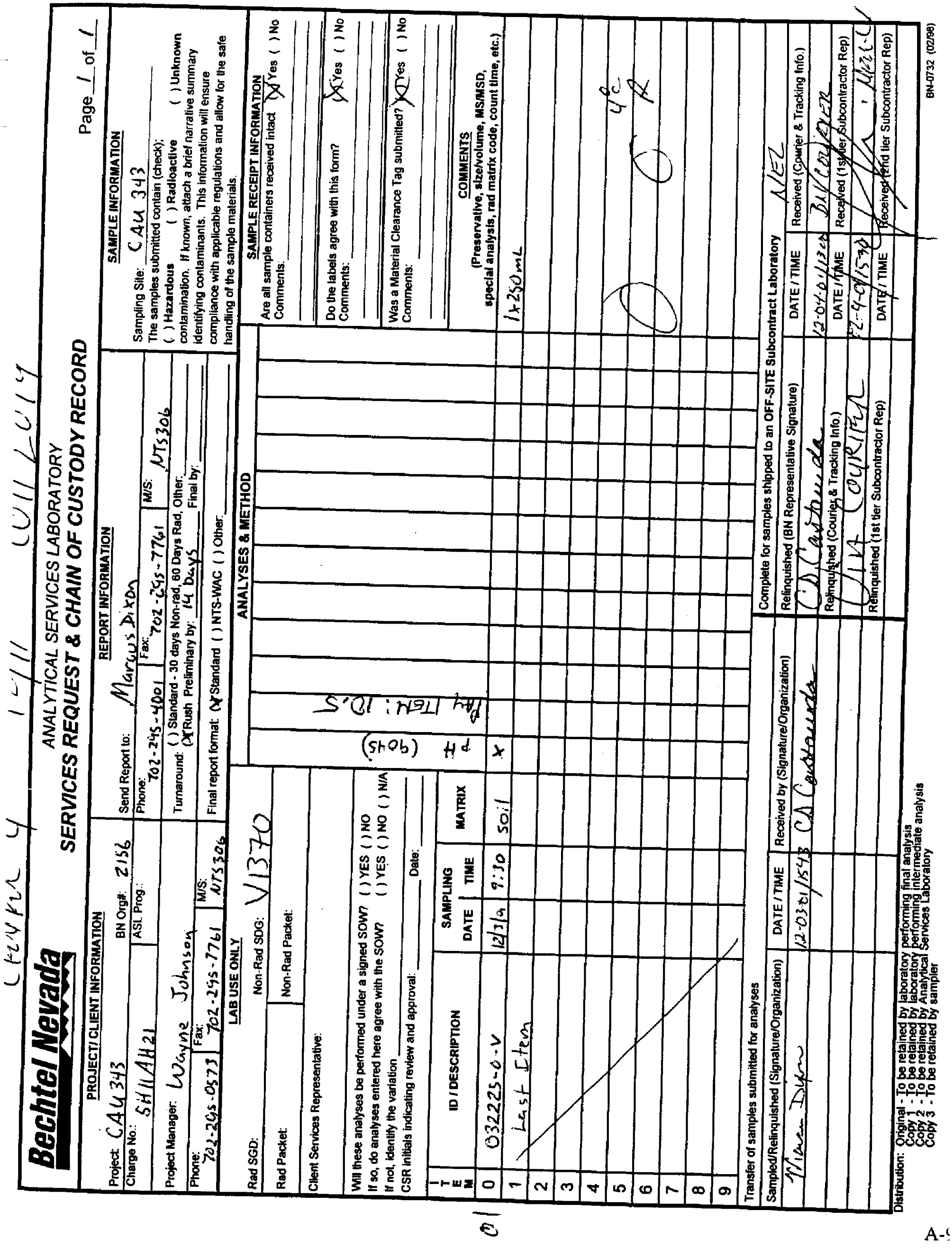


CLIENT:

Bechtel Nevada

P.O. Box 98521, M/S NTS273

Las Vegas, NV 89193-8521

ATTN:

Ted Redding

PROJECT NAME: V1358

NEL ORDER ID: L0111248

PROJECT NUMBER: 30033

Attached are the analytical resuits for samples in support of the above referenced project.

Samples submitted for this project were not sampled by NEL Laboratories. Samples were received by NEL in good condition, under chain of custody on 11/29/01.

Should you have any questions or comments, please feel free to contact our Client Services department at (702) 657-1010.

\section{Some results have been flagged as follows:}

J - This concentration should be considered an estimate due laboratory control sample failure.

$\mathrm{Jm}$ - This concentration should be considered an estimate due to probable matrix effects. The surrogate associated with this analyte was outside acceptance limits in the original analysis and upon reanalysis.

Some $Q A$ results have been flagged as follows:

J - This concentration should be considered an estimate due laboratory control sample failure.

ת - The batch MS and/or MSD were outside acceptance limits. The batch LCS was acceptable.

M2 - Matrix spike recovery was low, the method control sample recovery was acceptable.

R5 - RPD exceeded the laboratory control limit. Recovery met acceptance criteria.
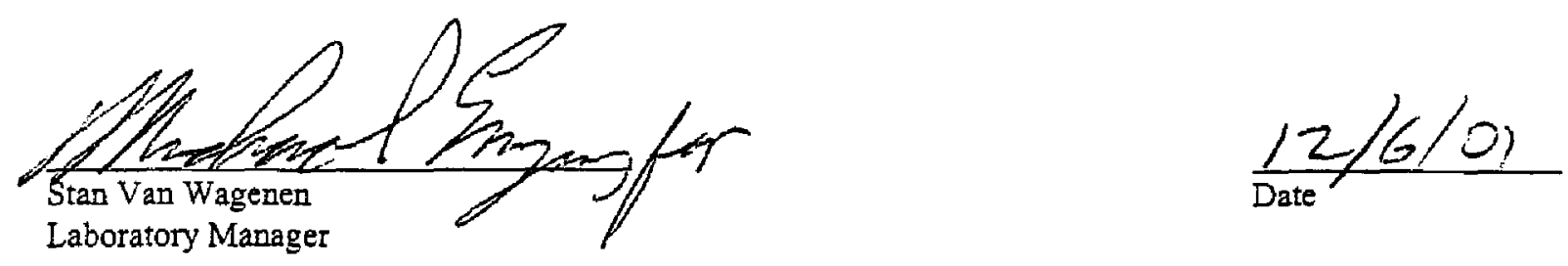

\section{CERTIFICATIONS:}

\begin{tabular}{llll}
\hline & Reno & Las Vegas & S. California \\
\cline { 2 - 4 } AZizona & AZ0520 & AZ0518 & AZ0605 \\
California & 1707 & 2002 & 2264 \\
$\begin{array}{l}\text { US Army Corps } \\
\text { of Engineers }\end{array}$ & Certified & Certified & \\
& & &
\end{tabular}

\begin{tabular}{llll} 
& Reno & Las Vegas & S. California \\
\cline { 2 - 4 } Idaho & Certified & Certified & \\
Montana & Certified & Certified & \\
Nevada & NV033 & NV052 & CA084 \\
L.A.C.S.D. & & & 10228
\end{tabular}




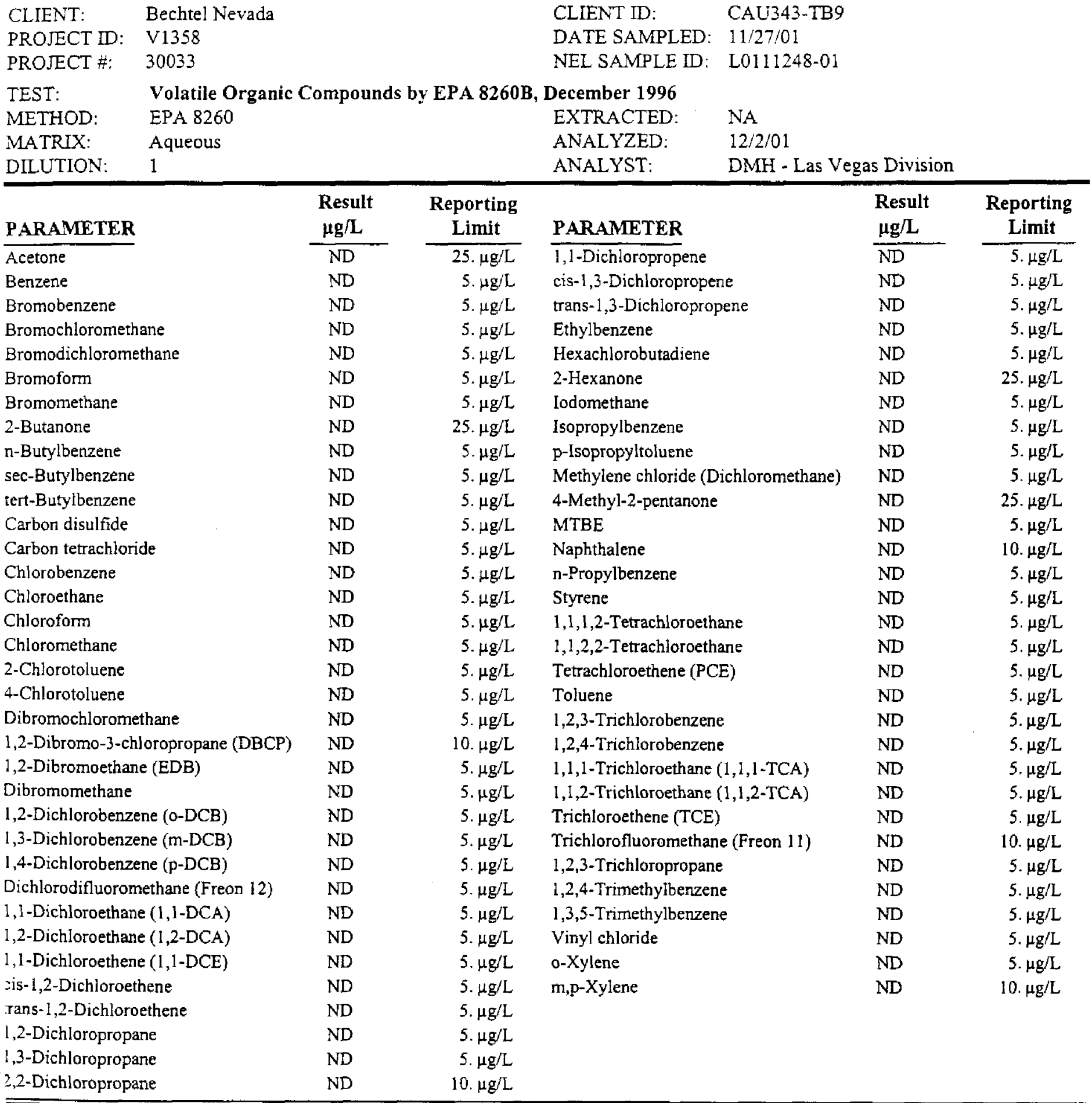

\section{UALITY CONTROL DATA:}

\section{Surrogate}

4-Bromofluorobenzene

Jibromofluoromethane

Toluene-d8

\section{$\%$ Recovery}

93

103

100

\section{Acceptable Range}

$76-111$

$88-114$

$95-108$

VD - Not Detected

This report shall not be reproduced except in full, without the written approval of the laboratory. 


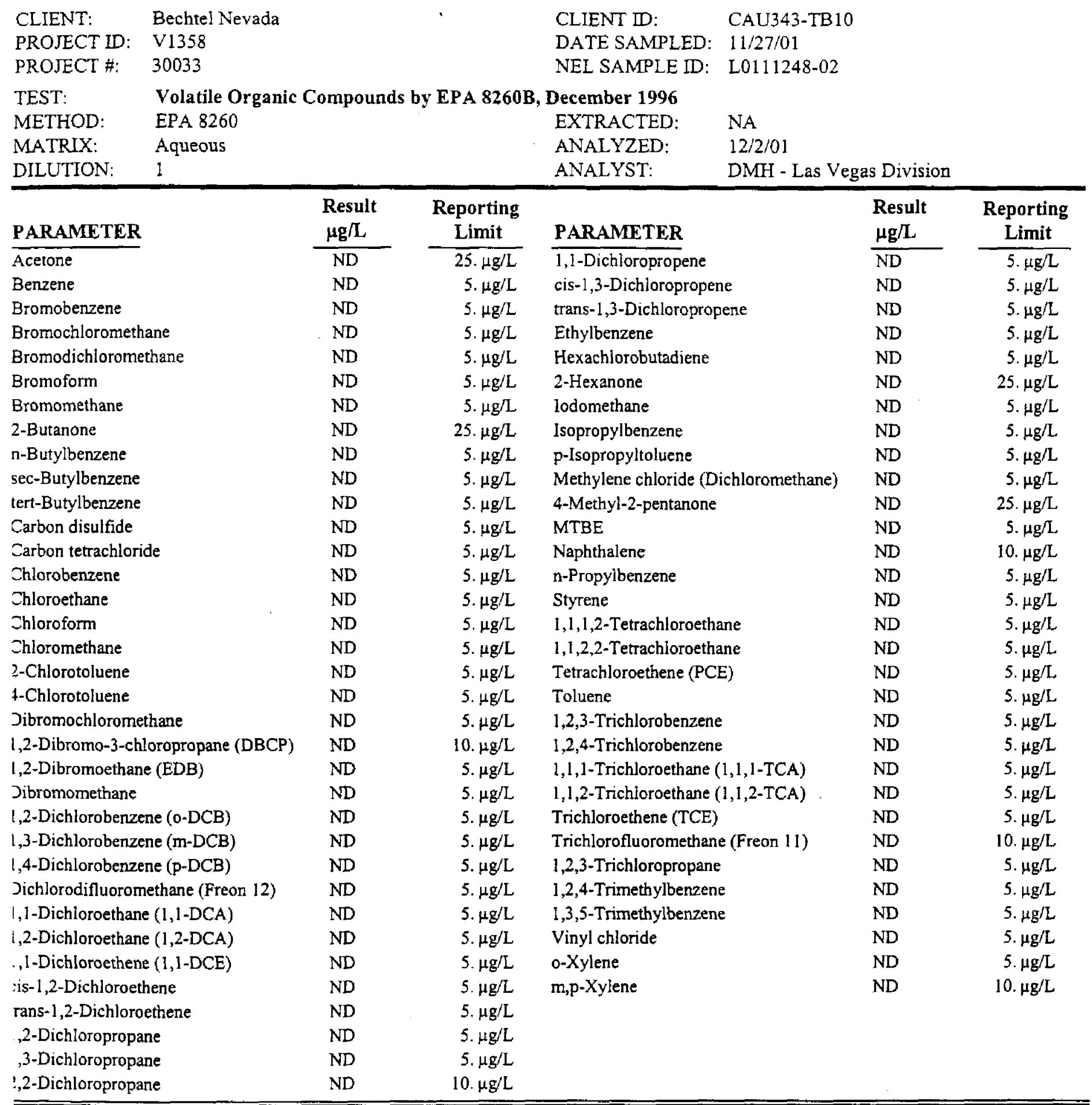

2UALITY CONTROL DATA:

iurrogate

-Bromofluorobenzene

Jibromofluoromethane

Toluene-d8

\section{$\%$ Recovery}

94

105

101
Acceptable Range

$76-111$

$88-114$

$95-108$

VD - Not Detected

This report shall not be reproduced except in full, without the written approval of the laboratory. 


$\begin{array}{llll}\text { CLIENT: } & \text { Bechtel Nevada } & \text { CLIENT ID: } & 032214-0-\mathrm{V} \\ \text { PROJECT ID: } & \text { V1358 } & \text { DATE SAMPLED: } & 11 / 27 / 01 \\ \text { PROJECT \#: } & 30033 & \text { NEL SAMPLE ID: } & \text { L0111248-03 }\end{array}$

Semi-Volatile Organic Compounds by EPA 8270C, Dec. 1996

METHOD EPA 8270

MATRIX: Solid

DILUTION: 1

EXTRACTED: $\quad 11 / 29 / 01$

ANALYZED: $\quad 11 / 30 / 01$

ANALYST: $\quad$ JRW - Las Vegas Division

\begin{tabular}{|c|c|c|c|c|c|}
\hline PARAMETER & $\begin{array}{l}\text { Result } \\
\mu \mathrm{g} / \mathrm{kg}\end{array}$ & $\begin{array}{c}\text { Reporting } \\
\text { Limit }\end{array}$ & PARAMETER & $\begin{array}{l}\text { Result } \\
\mu \mathrm{g} / \mathrm{kg}\end{array}$ & $\begin{array}{c}\text { Reporting } \\
\text { Limit }\end{array}$ \\
\hline Acenaphthene & ND & $330 . \mu \mathrm{g} / \mathrm{kg}$ & Dimethyl phthalate & $\mathrm{ND}$ & $330 . \mu \mathrm{g} / \mathrm{kg}$ \\
\hline Acenaphthylene & ND & $330 . \mu \mathrm{g} / \mathrm{kg}$ & 4,6-Dinitro-2-methyl phenol & ND & $330 . \mu \mathrm{g} / \mathrm{kg}$ \\
\hline Anitine & $\mathrm{ND}$ & $330 . \mu \mathrm{g} / \mathrm{kg}$ & 2,4-Dinitrotoluene (DNT) & $\mathrm{ND}$ & $330 . \mu \mathrm{g} / \mathrm{kg}$ \\
\hline Anthracene & ND & 330. $\mu \mathrm{g} / \mathrm{kg}$ & 2,6-Dinitrotoluene (DNT) & $\mathrm{ND}$ & $330 . \mu \mathrm{g} / \mathrm{kg}$ \\
\hline Azobenzene & ND & $330 . \mu \mathrm{g} / \mathrm{kg}$ & 2,4-Dinitrophenol & ND & $330 . \mu \mathrm{g} / \mathrm{kg}$ \\
\hline Benzo (a) anthracene & ND & $330 . \mu \mathrm{g} / \mathrm{kg}$ & Di-n-octyl phthalate & ND & $330 . \mu \mathrm{g} / \mathrm{kg}$ \\
\hline Benzo (b) fluoranthene & $\mathrm{ND}$ & $330 . \mu \mathrm{g} / \mathrm{kg}$ & Fluoranthene & ND & $330 . \mu \mathrm{g} / \mathrm{kg}$ \\
\hline Benzo (k) fluoranthene & ND & $330 . \mu \mathrm{g} / \mathrm{kg}$ & Fluorene & ND & $330 . \mu \mathrm{g} / \mathrm{kg}$ \\
\hline Benzoic Acid & $\mathrm{ND}$ & $330 . \mu \mathrm{g} / \mathrm{kg}$ & Hexachlorobenzene & $\mathrm{ND}$ & $330 . \mu \mathrm{g} / \mathrm{kg}$ \\
\hline Benzo $(\mathrm{g}, \mathrm{h}, \mathrm{i})$ perylene & ND & $330 . \mu \mathrm{g} / \mathrm{kg}$ & Hexachlorobutadiene & ND & $330 . \mu \mathrm{g} / \mathrm{kg}$ \\
\hline Benzo (a) pyrene & ND & $330 . \mu \mathrm{g} / \mathrm{kg}$ & Hexachlorocyclopentadiene & ND & $330 . \mu \mathrm{g} / \mathrm{kg}$ \\
\hline Benzyl alcohol & $\mathrm{ND}$ & $330 . \mu \mathrm{g} / \mathrm{kg}$ & Hexachloroethane & ND & $330 . \mu \mathrm{g} / \mathrm{kg}$ \\
\hline bis (2-Chloroethyl) ether & ND & 330. $\mu \mathrm{g} / \mathrm{kg}$ & Indeno $(1,2,3-c, d)$ pyrene & ND & $330 . \mu \mathrm{g} / \mathrm{kg}$ \\
\hline bis (2-Chloroethoxy) methane & ND & $330 . \mu \mathrm{g} / \mathrm{kg}$ & Isophorone & ND & $330 . \mu \mathrm{g} / \mathrm{kg}$ \\
\hline bis (2-chloroisopropyl) ether & ND & $330 . \mu \mathrm{g} / \mathrm{kg}$ & 2-Methylnaphthalene & ND & $330 . \mu \mathrm{g} / \mathrm{kg}$ \\
\hline bis (2-Ethylhexyl)phthalate & ND & $330 . \mu \mathrm{g} / \mathrm{kg}$ & 2-Methylphenol & ND & $330 . \mu \mathrm{g} / \mathrm{kg}$ \\
\hline Butyl benzyl phthalate & ND & $330 . \mu \mathrm{g} / \mathrm{kg}$ & 3,4-Methylphenol (isomeric pair) & ND & $330 . \mu \mathrm{g} / \mathrm{kg}$ \\
\hline 4-Bromophenyl phenyl ether & ND & $330 . \mu \mathrm{g} / \mathrm{kg}$ & Naphthalene & ND & $330 . \mu \mathrm{g} / \mathrm{kg}$ \\
\hline Jarbazole & ND & $330 . \mu \mathrm{g} / \mathrm{kg}$ & 2-Nitroaniline & ND & $330 . \mu \mathrm{g} / \mathrm{kg}$ \\
\hline 4-Chloroaniline & ND & 330. $\mu \mathrm{g} / \mathrm{kg}$ & 3-Nitroaniline & ND & $330 . \mu \mathrm{g} / \mathrm{kg}$ \\
\hline 7-Chloro-3-methyl phenol & ND & $330 . \mu \mathrm{g} / \mathrm{kg}$ & 4-Nitroaniline & ND & 330. $\mu \mathrm{g} / \mathrm{kg}$ \\
\hline 2-Chloronaphthalene & ND & $330 . \mu \mathrm{g} / \mathrm{kg}$ & Nitrobenzene & ND & $330 . \mu \mathrm{g} / \mathrm{kg}$ \\
\hline 2-Chlorophenol & ND & $330 . \mu \mathrm{g} / \mathrm{kg}$ & 2-Nitrophenol & ND & $330 . \mu \mathrm{g} / \mathrm{kg}$ \\
\hline 1-Chlorophenyl phenyl ether & ND & $330 . \mu \mathrm{g} / \mathrm{kg}$ & 4-Nitrophenol & ND & $330 . \mu \mathrm{g} / \mathrm{kg}$ \\
\hline Zhrysene & ND & $330 . \mu \mathrm{g} / \mathrm{kg}$ & N-Nitrosodi-n-propylamine & ND & $330 . \mu \mathrm{g} / \mathrm{kg}$ \\
\hline Jibenz $(a, h)$ anthracene & ND & $330 . \mu \mathrm{g} / \mathrm{kg}$ & N-Nitrosodimethylamine & ND & $330 . \mu \mathrm{g} / \mathrm{kg}$ \\
\hline Jibenzofuran & ND & $330 . \mu \mathrm{g} / \mathrm{kg}$ & N-Nitrosodiphenylamine & ND & $330 . \mu \mathrm{g} / \mathrm{kg}$ \\
\hline Ji-n-buty] phthalate & $\mathrm{ND}$ & $330 . \mu \mathrm{g} / \mathrm{kg}$ & Pentachlorophenol & ND & $330 . \mu \mathrm{g} / \mathrm{kg}$ \\
\hline !,2-Dichlorobenzene (o-DCB) & ND & $330 . \mu \mathrm{g} / \mathrm{kg}$ & Phenol & ND & $330 . \mu \mathrm{g} / \mathrm{kg}$ \\
\hline !,3-Dichlorobenzene (m-DCB) & ND & $330 . \mu \mathrm{g} / \mathrm{kg}$ & Phenanthrene & ND & $330 . \mu \mathrm{g} / \mathrm{kg}$ \\
\hline 1,4-Dichlorobenzene (p-DCB) & ND & $330 . \mu \mathrm{g} / \mathrm{kg}$ & Pyrene & ND & $330 . \mu \mathrm{g} / \mathrm{kg}$ \\
\hline ?,4-Dichlorophenol & $\mathrm{ND}$ & $330 . \mu \mathrm{g} / \mathrm{kg}$ & Pyridine & ND & $330 . \mu \mathrm{g} / \mathrm{kg}$ \\
\hline 3,3'-Dichlorobenzidine & ND & 330. $\mu \mathrm{g} / \mathrm{kg}$ & 1,2,4-Trichlorobenzene & ND & $330 . \mu \mathrm{g} / \mathrm{kg}$ \\
\hline Jiethyl phthaiate & ND & $330 . \mu \mathrm{g} / \mathrm{kg}$ & 2,4,5-Trichlorophenol & ND & $330 . \mu \mathrm{g} / \mathrm{kg}$ \\
\hline ',4-Dimethylphenol & ND & $330 . \mu \mathrm{g} / \mathrm{kg}$ & 2,4,6-Trichlorophenol & ND & $330 . \mu \mathrm{g} / \mathrm{kg}$ \\
\hline
\end{tabular}

2UALITY CONTROL DATA:

\section{jurrogate}

?,4,6-Tribromophenol

-Fluorobiphenyl

:-Fluorophenol

Jitrobenzene-d5

s-Terphenyl-d14

\section{\% Recovery}

68

46

45

43

62

\section{Acceptable Range}

$19-122$

$30-115$

$25-121$

$23-120$

$18-137$

JD - Not Detected

This report shall not be reproduced except in full, without the written approval of the laboratory. 


$\begin{array}{llll}\text { CLIENT: } & \text { Bechtel Nevada } & \text { CLIENT ID: } & 032207-0-\mathrm{V} \\ \text { PROJECT ID: } & \text { V1358 } & \text { DATE SAMPLED: } & 11 / 27 / 01 \\ \text { PROJECT \#: } & 30033 & \text { NEL SAMPLE ID: } & \text { L0111248-04 }\end{array}$

TEST:

METHOD

Volatile Organic Compounds by EPA 8260B, December 1996

MATRIX: EPA 8260

EXTRACTED:

NA

DILUTION

Soliđ

ANALYZED

$12 / 2 / 01$

ANALYST:

DMH - Las Vegas Division

\section{PARAMETER}

Acetone

Benzene

Bromobenzene

Bromochloromethane

Bromodichloromethane

Bromoform

Bromomethane

2-Butanone

n-Butylbenzene

sec-Butylbenzene

tert-Butylbenzene

Carbon disulfide

Carbon tetrachloride

Chlorobenzene

Chloroethane

Chloroform

Chloromethane

2-Chlorotoluene

4-Chlorotoluene

Dibromochloromethane

1,2-Dibromo-3-chloropropane (DBCP)

1,2-Dibromoethane (EDB)

Dibromomethane

1,2-Dichlorobenzene (o-DCB)

1,3-Dichlorobenzene (m-DCB)

1,4-Dichlorobenzene ( $p$-DCB)

Dichlorodifluoromethane (Freon 12)

1,1-Dichloroethane (1,1-DCA)

1,2-Dichloroethane (1,2-DCA)

1,1-Dichloroethene (1,1-DCE)

zis-1,2-Dichloroethene

rans-1,2-Dichloroethene

1,2-Dichloropropane

1,3-Dichloropropane

2,2-Dichloropropane

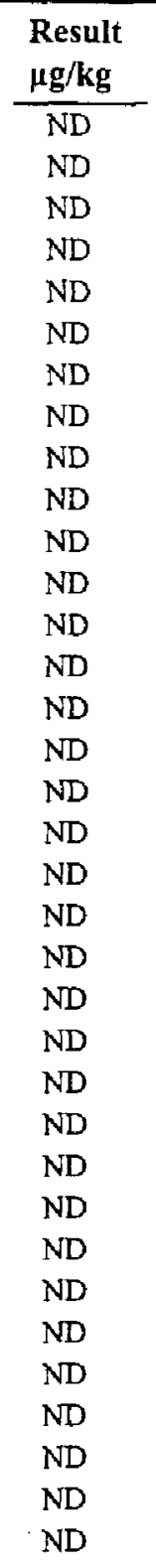

Reporting

Limit

5. $\mu \mathrm{g} / \mathrm{kg}$

5. $\mu \mathrm{g} / \mathrm{kg}$

5. $\mu \mathrm{g} / \mathrm{kg}$

5. $\mu \mathrm{g} / \mathrm{kg}$

5. $\mu \mathrm{g} / \mathrm{kg}$

$5 . \mu \mathrm{g} / \mathrm{kg}$

25. $\mu \mathrm{g} / \mathrm{kg}$

5. $\mu \mathrm{g} / \mathrm{kg}$

5. $\mu \mathrm{g} / \mathrm{kg}$

5. $\mu g / \mathrm{kg}$

5. $\mu \mathrm{g} / \mathrm{kg}$

5. $\mu \mathrm{g} / \mathrm{kg}$

$5 . \mu \mathrm{g} / \mathrm{kg}$

5. $\mu \mathrm{g} / \mathrm{kg}$

5. $\mu \mathrm{g} / \mathrm{kg}$

5. $\mu \mathrm{g} / \mathrm{kg}$

5. $\mu g / \mathrm{kg}$

5. $\mu \mathrm{g} / \mathrm{kg}$

5. $\mu \mathrm{g} / \mathrm{kg}$

10. $\mu \mathrm{g} / \mathrm{kg}$

5. $\mu \mathrm{g} / \mathrm{kg}$

5. $\mu \mathrm{g} / \mathrm{kg}$

5. $\mu \mathrm{g} / \mathrm{kg}$

5. $\mu \mathrm{g} / \mathrm{kg}$

5. $\mu \mathrm{g} / \mathrm{kg}$

5. $\mu \mathrm{g} / \mathrm{kg}$

5. $\mu \mathrm{g} / \mathrm{kg}$

5. $\mu \mathrm{g} / \mathrm{kg}$

$5 . \mu \mathrm{g} / \mathrm{kg}$

5. $\mu \mathrm{g} / \mathrm{kg}$

5. $\mu \mathrm{g} / \mathrm{kg}$

5. $\mu \mathrm{g} / \mathrm{kg}$

5. $\mu \mathrm{g} / \mathrm{kg}$

10. $\mu \mathrm{g} / \mathrm{kg}$

\section{PARAMETER}

1,1-Dichloropropene

cis-1,3-Dichloropropene

trans-1,3-Dichloropropene

Ethylbenzene

Hexachlorobutadiene

2-Hexanone

Iodomethane

Isopropylbenzene

p-Isopropyltoluene

Methylene chloride (Dichloromethane)

4-Methyl-2-pentanone

MTBE

Naphthaiene

n-Propylbenzene

Styrene

1,1,1,2-Tetrachloroethane

1,1,2,2-Tetrachloroethane

Tetrachloroethene (PCE)

Toluene

1,2,3-Trichlorobenzene

1,2,4-Trichlorobenzene

1,1,1-Trichloroethane (1,1,1-TCA)

1,1,2-Trichloroethane $(1,1,2-\mathrm{TCA})$

Trichloroethene (TCE)

Trichlorofluoromethane (Freon 11)

1,2,3-Trichloropropane

1,2,4-Trimethylbenzene

1,3,5-Trimethylbenzene

Vinyl chloride

o-Xylene

$\mathrm{m}, \mathrm{p}$-Xylene
Result

$\mu \mathrm{g} / \mathbf{k g}$

ND

ND

ND

ND

ND

ND

ND

ND

ND

ND

ND

ND

ND

ND

ND

ND

ND

ND

ND

ND

ND

ND

ND

ND

ND

ND

ND

ND

ND

ND

ND
Reporting

Limit

5. $\mu \mathrm{g} / \mathrm{kg}$

5. $\mu \mathrm{g} / \mathrm{kg}$

5. $\mu \mathrm{g} / \mathrm{kg}$

5. $\mu \mathrm{g} / \mathrm{kg}$

$5 . \mu \mathrm{g} / \mathrm{kg}$

$25 . \mu \mathrm{g} / \mathrm{kg}$

$5 . \mu \mathrm{g} / \mathrm{kg}$

5. $\mu \mathrm{g} / \mathrm{kg}$

5. $\mu \mathrm{g} / \mathrm{kg}$

$5 . \mu \mathrm{g} / \mathrm{kg}$

25. $\mu \mathrm{g} / \mathrm{kg}$

5. $\mu \mathrm{g} / \mathrm{kg}$

10. $\mu \mathrm{g} / \mathrm{kg}$

5. $\mu \mathrm{g} / \mathrm{kg}$

5. $\mu \mathrm{g} / \mathrm{kg}$

5. $\mu \mathrm{g} / \mathrm{kg}$

5. $\mu \mathrm{g} / \mathrm{kg}$

5. $\mu \mathrm{g} / \mathrm{kg}$

5. $\mu g / \mathrm{kg}$

5. $\mu \mathrm{g} / \mathrm{kg}$

$5 . \mu \mathrm{g} / \mathrm{kg}$

$5 . \mu \mathrm{g} / \mathrm{kg}$

5. $\mu \mathrm{g} / \mathrm{kg}$

5. $\mu \mathrm{g} / \mathrm{kg}$

10. $\mu \mathrm{g} / \mathrm{kg}$

5. $\mu \mathrm{g} / \mathrm{kg}$

5. $\mu \mathrm{g} / \mathrm{kg}$

5. $\mu \mathrm{g} / \mathrm{kg}$

$5 . \mu \mathrm{g} / \mathrm{kg}$

5. $\mu \mathrm{g} / \mathrm{kg}$

10. $\mu \mathrm{g} / \mathrm{kg}$

ZUALITY CONTROL DATA.

Surrogate

7-Bromofluorobenzene

Jibromofluoromethane

Toluene-d8

\section{$\%$ Recovery}

102

102

104
Acceptable Range

$74-121$

$80-120$

$81-117$

VD - Not Detected

This report shall not be reproduced except in full, without the written approval of the laboratory. 


$\begin{array}{llll}\text { CLIENT: } & \text { Bechtel Nevada } & \text { CLIENT ID: } & 032207-0-\mathrm{V} \\ \text { PROJECT ID: } & \text { V1358 } & \text { DATE SAMPLED: } 11 / 27 / 01 \\ \text { PROJECT \#: } & 30033 & \text { NEL SAMPLE ID: } & \text { L0111248-04 }\end{array}$

TEST: $\quad$ Semi-Volatile Organic Compounds by EPA 8270C, Dec. 1996

$\begin{array}{llll}\text { METHOD: } & \text { EPA } 8270 & \text { EXTRACTED: } & 11 / 29 / 01 \\ \text { MATRIX: } & \text { Solid } & \text { ANALYZED: } & 11 / 30 / 01 \\ \text { DILUTION: } & 1 & \text { ANALYST: } & \text { JRW - Las }\end{array}$

\begin{tabular}{|c|c|c|c|c|c|c|}
\hline PARAMETER & $\begin{array}{l}\text { Result } \\
\mu \mathrm{g} / \mathrm{kg}\end{array}$ & & $\begin{array}{c}\text { Reporting } \\
\text { Limit } \\
\end{array}$ & PARAMETER & $\begin{array}{l}\text { Result } \\
\mu \mathrm{g} / \mathrm{kg}\end{array}$ & $\begin{array}{c}\text { Reporting } \\
\text { Limit } \\
\end{array}$ \\
\hline Acenaphthene & ND & & $330 . \mu \mathrm{g} / \mathrm{kg}$ & Dimethyl phthalate & $\overline{N D}$ & $\overline{330 . \mu \mathrm{g} / \mathrm{kg}}$ \\
\hline Acenaphthylene & $\mathrm{ND}$ & & $330 . \mu \mathrm{g} / \mathrm{kg}$ & 4,6-Dinitro-2-methyl phenol & $\mathrm{ND}$ & $330 . \mu \mathrm{g} / \mathrm{kg}$ \\
\hline Aniline & ND & & $330 . \mu \mathrm{g} / \mathrm{kg}$ & 2,4-Dinitrotoluene (DNT) & $\mathrm{ND}$ & $330 . \mu \mathrm{g} / \mathrm{kg}$ \\
\hline Anthracene & ND & & $330 . \mu \mathrm{g} / \mathrm{kg}$ & 2,6-Dinitrotoluene (DNT) & ND & $330 . \mu \mathrm{g} / \mathrm{kg}$ \\
\hline Azobenzene & ND & & $330 . \mu \mathrm{g} / \mathrm{kg}$ & 2,4-Dinitrophenol & ND & $330 . \mu \mathrm{g} / \mathrm{kg}$ \\
\hline Benzo (a) anthracene & $\mathrm{ND}$ & & $330 . \mu \mathrm{g} / \mathrm{kg}$ & Di-n-octyl phthalate & $\mathrm{ND}$ & $330 . \mu \mathrm{g} / \mathrm{kg}$ \\
\hline Benzo (b) fluoranthene & ND & & $330 . \mu \mathrm{g} / \mathrm{kg}$ & Fluoranthene & ND & 330. $\mu \mathrm{g} / \mathrm{kg}$ \\
\hline Benzo $(k)$ fluoranthene & $\mathrm{ND}$ & & $330 . \mu \mathrm{g} / \mathrm{kg}$ & Fluorene & ND & 330. $\mu \mathrm{g} / \mathrm{kg}$ \\
\hline Benzoic Acid & ND & $\mathrm{J}$ & $330 . \mu \mathrm{g} / \mathrm{kg}$ & Hexachlorobenzene & ND & 330. $\mu \mathrm{g} / \mathrm{kg}$ \\
\hline Benzo $(\mathrm{g}, \mathrm{h}, \mathrm{i})$ perylene & ND & & $330 . \mu \mathrm{g} / \mathrm{kg}$ & Hexachlorobutadiene & ND & 330. $\mu \mathrm{g} / \mathrm{kg}$ \\
\hline Benzo (a) pyrene & ND & & $330 . \mu \mathrm{g} / \mathrm{kg}$ & Hexachlorocyclopentadiene & ND & $330 . \mu \mathrm{g} / \mathrm{kg}$ \\
\hline Benzyl aicohol & ND & & $330 . \mu \mathrm{g} / \mathrm{kg}$ & Hexachloroethane & ND & 330. $\mu \mathrm{g} / \mathrm{kg}$ \\
\hline bis (2-Chloroethyl) ether & ND & & $330 . \mu \mathrm{g} / \mathrm{kg}$ & Indeno $(1,2,3-c, d)$ pyrene & ND & 330. $\mu \mathrm{g} / \mathrm{kg}$ \\
\hline bis (2-Chloroethoxy) methane & ND & & $330 . \mu \mathrm{g} / \mathrm{kg}$ & Isophorone & ND & $330 . \mu \mathrm{g} / \mathrm{kg}$ \\
\hline bis (2-chloroisopropyl) ether & ND & & $330 . \mu \mathrm{g} / \mathrm{kg}$ & 2-Methylnaphthalene & ND & $330 . \mu g / k g$ \\
\hline bis (2-Ethylhexyl)phthalate & ND & & $330 . \mu \mathrm{g} / \mathrm{kg}$ & 2-Methylphenol & ND & 330. $\mu \mathrm{g} / \mathrm{kg}$ \\
\hline Butyl benzyl phthalate & ND & & $330 . \mu \mathrm{g} / \mathrm{kg}$ & 3,4-Methylphenol (isomeric pair) & ND & 330. $\mu \mathrm{g} / \mathrm{kg}$ \\
\hline 4-Bromophenyl phenyl ether & ND & & $330 . \mu \mathrm{g} / \mathrm{kg}$ & Naphthalene & ND & $330 . \mu g / \mathrm{kg}$ \\
\hline Carbazole & ND & & $330 . \mu \mathrm{g} / \mathrm{kg}$ & 2-Nitroaniline & ND & $330 . \mu \mathrm{g} / \mathrm{kg}$ \\
\hline 4-Chloroaniline & ND & & $330 . \mu \mathrm{g} / \mathrm{kg}$ & 3-Nitroaniline & ND & $330 . \mu \mathrm{g} / \mathrm{kg}$ \\
\hline 4-Chloro-3-methyl phenol & ND & & $330 . \mu \mathrm{g} / \mathrm{kg}$ & 4-Nitroaniline & ND & $330 . \mu \mathrm{g} / \mathrm{kg}$ \\
\hline 2-Chloronaphthalene & $\mathrm{ND}$ & & $330 . \mu \mathrm{g} / \mathrm{kg}$ & Nitrobenzene & ND & $330 . \mu \mathrm{g} / \mathrm{kg}$ \\
\hline 2-Chlorophenol & ND & & $330 . \mu \mathrm{g} / \mathrm{kg}$ & 2-Nitrophenol & ND & $330 . \mu \mathrm{g} / \mathrm{kg}$ \\
\hline 4-Chlorophenyl phenyl ether & ND & & $330 . \mu \mathrm{g} / \mathrm{kg}$ & 4-Nitrophenol & ND & 330. $\mu \mathrm{g} / \mathrm{kg}$ \\
\hline Chrysene & ND & & $330 . \mu \mathrm{g} / \mathrm{kg}$ & N-Nitrosodi-n-propylamine & $\mathrm{ND}$ & 330. $\mu \mathrm{g} / \mathrm{kg}$ \\
\hline Dibenz $(\mathrm{a}, \mathrm{h})$ anthracene & ND & & $330, \mu \mathrm{g} / \mathrm{kg}$ & N-Nitrosodimethylamine & ND & 330. $\mu \mathrm{g} / \mathrm{kg}$ \\
\hline Dibenzofuran & ND & & $330 . \mu \mathrm{g} / \mathrm{kg}$ & $\mathrm{N}$-Nitrosodiphenylamine & ND & $330 . \mu \mathrm{g} / \mathrm{kg}$ \\
\hline Di-n-butyl phthalate & ND & & $330 . \mu \mathrm{g} / \mathrm{kg}$ & Pentachlorophenol & ND & $330 . \mu \mathrm{g} / \mathrm{kg}$ \\
\hline 1,2-Dichlorobenzene (o-DCB) & ND & & $330 . \mu \mathrm{g} / \mathrm{kg}$ & Phenol & ND & 330. $\mu \mathrm{g} / \mathrm{kg}$ \\
\hline 1,3-Dichlorobenzene (m-DCB) & ND & & $330 . \mu \mathrm{g} / \mathrm{kg}$ & Phenarthrene & ND & $330 . \mu \mathrm{g} / \mathrm{kg}$ \\
\hline 1,4-Dichlorobenzene (p-DCB) & ND & & $330 . \mu \mathrm{g} / \mathrm{kg}$ & Pyrene & ND & $330 . \mu \mathrm{g} / \mathrm{kg}$ \\
\hline 2,4-Dichlorophenol & ND & & $330 . \mu \mathrm{g} / \mathrm{kg}$ & Pyridine & ND & 330. $\mu \mathrm{g} / \mathrm{kg}$ \\
\hline 3,3'-Dichlorobenzidine & ND & & $330 . \mu \mathrm{g} / \mathrm{kg}$ & 1,2,4-Trichlorobenzene & ND & 330. $\mu \mathrm{g} / \mathrm{kg}$ \\
\hline Diethyl phthalate & ND & & $330 . \mu \mathrm{g} / \mathrm{kg}$ & 2,4,5-Trichlorophenol & ND & 330. $\mu \mathrm{g} / \mathrm{kg}$ \\
\hline 2,4-Dimethylphenol & ND & & $330 . \mu \mathrm{g} / \mathrm{kg}$ & 2,4,6-Trichlorophenol & ND & $330 . \mu \mathrm{g} / \mathrm{kg}$ \\
\hline
\end{tabular}

QUALITY CONTROL DATA:

\section{Surrogate}

2,4,6-Tribromophenol

2-Fluorobiphenyl

2-Fluorophenol

Nitrobenzene-d5

ว-Terphenyl-d14
\% Recovery

95

59

59

55

86

\section{Acceptable Range}

$19-122$
$30-115$
$25-121$
$23-120$
$18-137$

ND - Not Detected

This report shall not be reproduced except in full, without the written approval of the laboratory. 


$\begin{array}{llll}\text { CLIENT: } & \text { Bechtel Nevada } & \text { CLIENT ID: } & \text { Method Blank } \\ \text { PROJECT ID: } & \text { V1358 } & \text { DATE SAMPLED: } & \text { NA } \\ \text { PROJECT \#: } & 30033 & \text { NEL SAMPLE ID: } & \text { 011129-8270S-BLK }\end{array}$

TEST:

Semi-Volatile Organic Compounds by EPA 8270C, Dec. 1996

METHOD: $\quad$ EPA 8270

ANALYST:

MATRIX: Solid

EXTRACTED: $\quad 11 / 29 / 01$

ANALYZED: $\quad 11 / 30 / 01$

\begin{tabular}{|c|c|c|c|c|c|}
\hline PARAMETER & $\begin{array}{l}\text { Result } \\
\mu \mathrm{g} / \mathrm{kg}\end{array}$ & $\begin{array}{l}\text { Reporting } \\
\text { Limit }\end{array}$ & PARAMETER & $\begin{array}{l}\text { Result } \\
\mu \mathrm{g} / \mathrm{kg}\end{array}$ & $\begin{array}{l}\text { Reporting } \\
\text { Limit }\end{array}$ \\
\hline Acenaphthene & ND & $330 \mu \mathrm{g} / \mathrm{kg}$ & Diethyl phthalate & ND & $330 \mu \mathrm{g} / \mathrm{kg}$ \\
\hline Acenaphthylene & ND & $330 \mu \mathrm{g} / \mathrm{kg}$ & 2,4-Dimethylphenol & ND & $330 \mu \mathrm{g} / \mathrm{kg}$ \\
\hline Aniline & ND & $330 \mu \mathrm{g} / \mathrm{kg}$ & Dimethyl phthalate & ND & $330 \mu \mathrm{g} / \mathrm{kg}$ \\
\hline Anthracene & ND & $330 \mu \mathrm{g} / \mathrm{kg}$ & 4,6-Dinitro-2-methyl phenol & ND & $330 \mu \mathrm{g} / \mathrm{kg}$ \\
\hline Azobenzene & ND & $330 \mu \mathrm{g} / \mathrm{kg}$ & 2,4-Dinitrotoluene (DNT) & $\mathrm{ND}$ & $330 \mu \mathrm{g} / \mathrm{kg}$ \\
\hline Benzo (a) anthracene & ND & $330 \mu \mathrm{g} / \mathrm{kg}$ & 2,6-Dinitrotoluene (DNT) & ND & $330 \mu \mathrm{g} / \mathrm{kg}$ \\
\hline Benzo (b) fluoranthene & ND & $330 \mu \mathrm{g} / \mathrm{kg}$ & 2,4-Dinitrophenol & ND & $330 \mu \mathrm{g} / \mathrm{kg}$ \\
\hline Benzo $(\mathrm{k})$ fluoranthene & ND & $330 \mathrm{\mu g} / \mathrm{kg}$ & Di-n-octyl phthalate & ND & $330 \mu \mathrm{g} / \mathrm{kg}$ \\
\hline Benzoic Acid & ND & $330 \mu \mathrm{g} / \mathrm{kg}$ & Fluoranthene & ND & $330 \mu \mathrm{g} / \mathrm{kg}$ \\
\hline Benzo $(\mathrm{g}, \mathrm{h}, \mathrm{i})$ perylene & ND & $330 \mu \mathrm{g} / \mathrm{kg}$ & Fluorene & ND & $330 \mu \mathrm{g} / \mathrm{kg}$ \\
\hline Benzo (a) pyrene & ND & $330 \mu \mathrm{g} / \mathrm{kg}$ & Hexachlorobenzene & ND & $330 \mu \mathrm{g} / \mathrm{kg}$ \\
\hline Benzyl alcohol & ND & $330 \mu \mathrm{g} / \mathrm{kg}$ & Hexachlorobutadiene & ND & $330 \mu g / k g$ \\
\hline bis (2-Chloroethyl) ether & ND & $330 \mu \mathrm{g} / \mathrm{kg}$ & Hexachlorocyclopentadiene & ND & $330 \mu \mathrm{g} / \mathrm{kg}$ \\
\hline bis (2-Chloroethoxy) methane & ND & $330 \mu \mathrm{g} / \mathrm{kg}$ & Hexachloroethane & ND & $330 \mu \mathrm{g} / \mathrm{kg}$ \\
\hline bis (2-chloroisopropyl) ether & ND & $330 \mu \mathrm{g} / \mathrm{kg}$ & Indeno $(1,2,3-c, d)$ pyrene & ND & $330 \mu \mathrm{g} / \mathrm{kg}$ \\
\hline bis (2-Ethylhexyl)phthalate & ND & $330 \mu \mathrm{g} / \mathrm{kg}$ & Isophorone & ND & $330 \mu \mathrm{g} / \mathrm{kg}$ \\
\hline Butyl benzyl phthalate & ND & $330 \mu \mathrm{g} / \mathrm{kg}$ & 2-Methylnaphthalene & ND & $330 \mu \mathrm{g} / \mathrm{kg}$ \\
\hline 4-Bromophenyl phenyl ether & ND & $330 \mu \mathrm{g} / \mathrm{kg}$ & 2-Methylphenol & ND & $330 \mu \mathrm{g} / \mathrm{kg}$ \\
\hline Carbazole & ND & $330 \mu \mathrm{g} / \mathrm{kg}$ & 3,4-Methylphenol (isomeric pair) & ND & $330 \mu \mathrm{g} / \mathrm{kg}$ \\
\hline 4-Chloroaniline & ND & $330 \mu \mathrm{g} / \mathrm{kg}$ & Naphthalene & ND & $330 \mu \mathrm{g} / \mathrm{kg}$ \\
\hline 4-Chloro-3-methyl phenol & ND & $330 \mu \mathrm{g} / \mathrm{kg}$ & 2-Nitroaniline & ND & $330 \mu \mathrm{g} / \mathrm{kg}$ \\
\hline 2-Chloronaphthalene & $\mathrm{ND}$ & $330 \mu \mathrm{g} / \mathrm{kg}$ & 3-Nitroaniline & ND & $330 \mu \mathrm{g} / \mathrm{kg}$ \\
\hline ?-Chlorophenol & ND & $330 \mu \mathrm{g} / \mathrm{kg}$ & 4-Nitroaniline & ND & $330 \mu \mathrm{g} / \mathrm{kg}$ \\
\hline 4-Chlorophenyl phenyl ether & ND & $330 \mu \mathrm{g} / \mathrm{kg}$ & Nitrobenzene & ND & $330 \mu \mathrm{g} / \mathrm{kg}$ \\
\hline Thrysene & ND & $330 \mu \mathrm{g} / \mathrm{kg}$ & 2-Nitrophenol & ND & $330 \mu \mathrm{g} / \mathrm{kg}$ \\
\hline Jibenz $(a, h)$ anthracene & ND & $330 \mu \mathrm{g} / \mathrm{kg}$ & 4-Nitrophenol & ND & $330 \mu \mathrm{g} / \mathrm{kg}$ \\
\hline Jibenzofuran & ND & $330 \mu \mathrm{g} / \mathrm{kg}$ & N-Nitrosodi-n-propylamine & ND & $330 \mu \mathrm{g} / \mathrm{kg}$ \\
\hline Ji-n-butyl phthalate & ND & $330 \mu \mathrm{g} / \mathrm{kg}$ & $\mathrm{N}$-Nitrosodimethylamine & ND & $330 \mu \mathrm{g} / \mathrm{kg}$ \\
\hline 1,2-Dichlorobenzene (o-DCB) & ND & $330 \mu \mathrm{g} / \mathrm{kg}$ & N-Nitrosodiphenylamine & ND & $330 \mu \mathrm{g} / \mathrm{kg}$ \\
\hline 1,3-Dichlorobenzene (m-DCB) & ND & $330 \mu \mathrm{g} / \mathrm{kg}$ & Pentachlorophenol & $\mathrm{ND}$ & $330 \mu \mathrm{g} / \mathrm{kg}$ \\
\hline 1,4-Dichlorobenzene (p-DCB) & ND & $330 \mu \mathrm{g} / \mathrm{kg}$ & Phenol & ND & $330 \mu \mathrm{g} / \mathrm{kg}$ \\
\hline 2,4-Dichlorophenol & $\mathrm{ND}$ & $330 \mu \mathrm{g} / \mathrm{kg}$ & Phenanthrene & ND & $330 \mu \mathrm{g} / \mathrm{kg}$ \\
\hline 3,3'-Dichlorobenzidine & ND & $330 \mu \mathrm{g} / \mathrm{kg}$ & Pyrene & ND & $330 \mu \mathrm{g} / \mathrm{kg}$ \\
\hline
\end{tabular}

\section{QUALITY CONTROL DATA:}

\section{Surrogate}

2,4,6-Tribromophenol

2-Fluorobiphenyl

2-Fluorophenol

Nitrobenzene-d5

p-Terphenyl-d14

Phenol-d6

\begin{tabular}{c} 
\% Recovery \\
\hline 74 \\
57 \\
57 \\
57 \\
73
\end{tabular}

Acceptable Range

$19-122$

$30-115$

$25-121$

$23-120$

$18-137$

$24-113$

ND - Not Detected

This report shall not be reproduced except in full, without the written approval of the laboratory. 


$\begin{array}{llll}\text { CLIENT: } & \text { Bechtel Nevada } & \text { CLIENT ID: } & \text { Method Blank } \\ \text { PROJECT ID: } & \text { V1358 } & \text { DATE SAMPLED: } & \text { NA } \\ \text { PROJECT \#: } & 30033 & \text { NEL SAMPLE ID: } & 011202 A Q 60 \_2 A-B L K\end{array}$

TEST:

METHOD:

Volatile Organic Compounds by EPA 8260B, December 1996

MATRIX:

EPA 8260

Aqueous
ANALYST: DMH - Las Vegas Division

EXTRACTED: NA

ANALYZED: $12 / 2 / 01$

\begin{tabular}{|c|c|c|c|c|c|}
\hline PARAMETER & $\begin{array}{c}\text { Result } \\
\mu \mathrm{g} / \mathrm{L}\end{array}$ & $\begin{array}{l}\text { Reporting } \\
\text { Limit }\end{array}$ & PARAMETER & $\begin{array}{l}\text { Result } \\
\mu \mathrm{g} / \mathrm{L}\end{array}$ & $\begin{array}{l}\text { Reporting } \\
\text { Limit }\end{array}$ \\
\hline Acetone & ND & $25 \mu \mathrm{g} / \mathrm{L}$ & 1,3-Dichloropropane & ND & $5 \mu \mathrm{g} / \mathrm{L}$ \\
\hline Benzene & ND & $5 \mu \mathrm{g} / \mathrm{L}$ & 2,2-Dichloropropane & ND & $10 \mu \mathrm{g} / \mathrm{L}$ \\
\hline Bromobenzene & ND & $5 \mu \mathrm{g} / \mathrm{L}$ & 1,1-Dichloropropene & ND & $5 \mu \mathrm{g} / \mathrm{L}$ \\
\hline Bromochloromethane & ND & $5 \mu \mathrm{g} / \mathrm{L}$ & cis-1,3-Dichloropropene & ND & $5 \mu \mathrm{g} / \mathrm{L}$ \\
\hline Bromodichloromethane & ND & $5 \mu \mathrm{g} / \mathrm{L}$ & trans-1,3-Dichloropropene & ND & $5 \mu \mathrm{g} / \mathrm{L}$ \\
\hline Bromoform & ND & $5 \mu \mathrm{g} / \mathrm{L}$ & Ethylbenzene & ND & $5 \mu \mathrm{g} / \mathrm{L}$ \\
\hline Bromomethane & ND & $5 \mu \mathrm{g} / \mathrm{L}$ & Hexachlorobutadiene & ND & $5 \mu \mathrm{g} / \mathrm{L}$ \\
\hline 2-Butanone & ND & $25 \mu \mathrm{g} / \mathrm{L}$ & 2-Hexanone & ND & $25 \mu \mathrm{g} / \mathrm{L}$ \\
\hline n-Butylbenzene & ND & $5 \mu \mathrm{g} / \mathrm{L}$ & Iodomethane & ND & $5 \mu \mathrm{g} / \mathrm{L}$ \\
\hline sec-Butylbenzene & ND & $5 \mu \mathrm{g} / \mathrm{L}$ & Isopropylbenzene & ND & $5 \mu \mathrm{g} / \mathrm{L}$ \\
\hline tert-Butylbenzene & ND & $5 \mu \mathrm{g} / \mathrm{L}$ & p-Isopropyltoluene & ND & $5 \mu \mathrm{g} / \mathrm{L}$ \\
\hline Carbon disulfide & ND & $5 \mu \mathrm{g} / \mathrm{L}$ & Methylene chloride (Dichloromethane) & ND & $5 \mu \mathrm{g} / \mathrm{L}$ \\
\hline Carbon tetrachloride & ND & $5 \mu g / L$ & 4-Methyl-2-pentanone & $\mathrm{ND}$ & $25 \mu \mathrm{g} / \mathrm{L}$ \\
\hline Chilorobenzene & ND & $5 \mu \mathrm{g} / \mathrm{L}$ & MTBE & ND & $5 \mu \mathrm{g} / \mathrm{L}$ \\
\hline Chloroethane & ND & $5 \mu \mathrm{g} / \mathrm{L}$ & Naphthalene & ND & $10 \mu \mathrm{g} / \mathrm{L}$ \\
\hline Chloroform & ND & $5 \mu \mathrm{g} / \mathrm{L}$ & n-Propylbenzene & $\mathrm{ND}$ & $5 \mu \mathrm{g} / \mathrm{L}$ \\
\hline Chloromethane & ND & $5 \mu \mathrm{g} / \mathrm{L}$ & Styrene & ND & $5 \mu \mathrm{g} / \mathrm{L}$ \\
\hline 2-Chlorotoluene & ND & $5 \mu \mathrm{g} / \mathrm{L}$ & 1,1,1,2-Tetrachloroethane & ND & $5 \mu \mathrm{g} / \mathrm{L}$ \\
\hline 4-Chlorotoluene & ND & $5 \mu \mathrm{g} / \mathrm{L}$ & $1,1,2,2$-Tetrachloroethane & $\mathrm{ND}$ & $5 \mu \mathrm{g} / \mathrm{L}$ \\
\hline Dibromochloromethane & ND & $5 \mu \mathrm{g} / \mathrm{L}$ & Tetrachloroethene (PCE) & ND & $5 \mu \mathrm{g} / \mathrm{L}$ \\
\hline 1,2-Dibromo-3-chloropropane (DBCP) & ND & $10 \mu \mathrm{g} / \mathrm{L}$ & Toluene & ND & $5 \mu \mathrm{g} / \mathrm{L}$ \\
\hline 1,2-Dibromoethane (EDB) & ND & $5 \mu \mathrm{g} / \mathrm{L}$ & 1,2,3-Trichlorobenzene & ND & $5 \mu \mathrm{g} / \mathrm{L}$ \\
\hline Dibromomethane & ND & $5 \mu g / L$ & 1,2,4-Trichlorobenzene & ND & $5 \mu \mathrm{g} / \mathrm{L}$ \\
\hline 1,2-Dichlorobenzene (o-DCB) & ND & $5 \mu \mathrm{g} / \mathrm{L}$ & 1,1,1-Trichloroethane (1,1,1-TCA) & ND & $5 \mu \mathrm{g} / \mathrm{L}$ \\
\hline 1,3-Dichlorobenzene (m-DCB) & ND & $5 \mu \mathrm{g} / \mathrm{L}$ & 1,1,2-Trichioroethane $(1,1,2-\mathrm{TCA})$ & ND & $5 \mu \mathrm{g} / \mathrm{L}$ \\
\hline 1,4-Dichlorobenzene ( $\mathrm{p}$-DCB) & ND & $5 \mu \mathrm{g} / \mathrm{L}$ & Trichloroethene (TCE) & ND & $5 \mu \mathrm{g} / \mathrm{L}$ \\
\hline Dichlorodifluoromethane (Freon 12) & ND & $5 \mu \mathrm{g} / \mathrm{L}$ & Trichlorofluoromethane (Freon 11) & ND & $10 \mu \mathrm{g} / \mathrm{L}$ \\
\hline 1,1-Dichloroethane (1,1-DCA) & ND & $5 \mu \mathrm{g} / \mathrm{L}$ & 1,2,3-Trichloropropane & ND & $5 \mu \mathrm{g} / \mathrm{L}$ \\
\hline 1,2-Dichloroethane (1,2-DCA) & ND & $5 \mu \mathrm{g} / \mathrm{L}$ & 1,2,4-Trimethylbenzene & ND & $5 \mu \mathrm{g} / \mathrm{L}$ \\
\hline 1,1-Dichloroethene (1,1-DCE) & ND & $5 \mu \mathrm{g} / \mathrm{L}$ & 1,3,5-Trimethylbenzene & ND & $5 \mu g / L$ \\
\hline cis-1,2-Dichloroethene & ND & $5 \mu \mathrm{g} / \mathrm{L}$ & Vinyl chloride & ND & $5 \mu \mathrm{g} / \mathrm{L}$ \\
\hline trans-1,2-Dichloroethene & ND & $5 \mu \mathrm{g} / \mathrm{L}$ & o-Xylene & ND & $5 \mu \mathrm{g} / \mathrm{L}$ \\
\hline 1,2-Dichloropropane & ND & $5 \mu \mathrm{g} / \mathrm{L}$ & $\mathrm{m}, \mathrm{p}$-Xylene & ND & $10 \mu \mathrm{g} / \mathrm{L}$ \\
\hline
\end{tabular}

QUALITY CONTROL DATA:

\section{Surrogate}

4-Bromofluorobenzene

Dibromofluoromethane

Toluene-d8

\begin{tabular}{c} 
\% Recovery \\
\hline 88 \\
100 \\
98
\end{tabular}

98
Acceptable Range

$76-111$

$88-114$

$95-108$

ND - Not Detected

This report shall not be reproduced except in full, without the written approval of the laboratory. 


$\begin{array}{llcl}\text { CLIENT: } & \text { Bechtel Nevada } & \text { CLIENT ID: } & \text { Method Blank } \\ \text { PROJECT ID: } & \text { V1358 } & \text { DATE SAMPLED: } & \text { NA } \\ \text { PROJECT \#: } & 30033 & \text { NEL SAMPLE ID: } & 0112025 D 60 \_1 A-B L K \\ \text { TEST: } & \text { Volatile Organic Compounds by EPA 8260B, } & \text { December 1996 } & \\ \text { METHOD: } & \text { EPA 8260 } & \text { ANALYST: } & \text { DMH - Las Vegas Division } \\ \text { MATRIX: } & \text { Solid } & \text { EXTRACTED: } & \text { NA } \\ & & \text { ANALYZED: } & 12 / 2 / 01\end{array}$

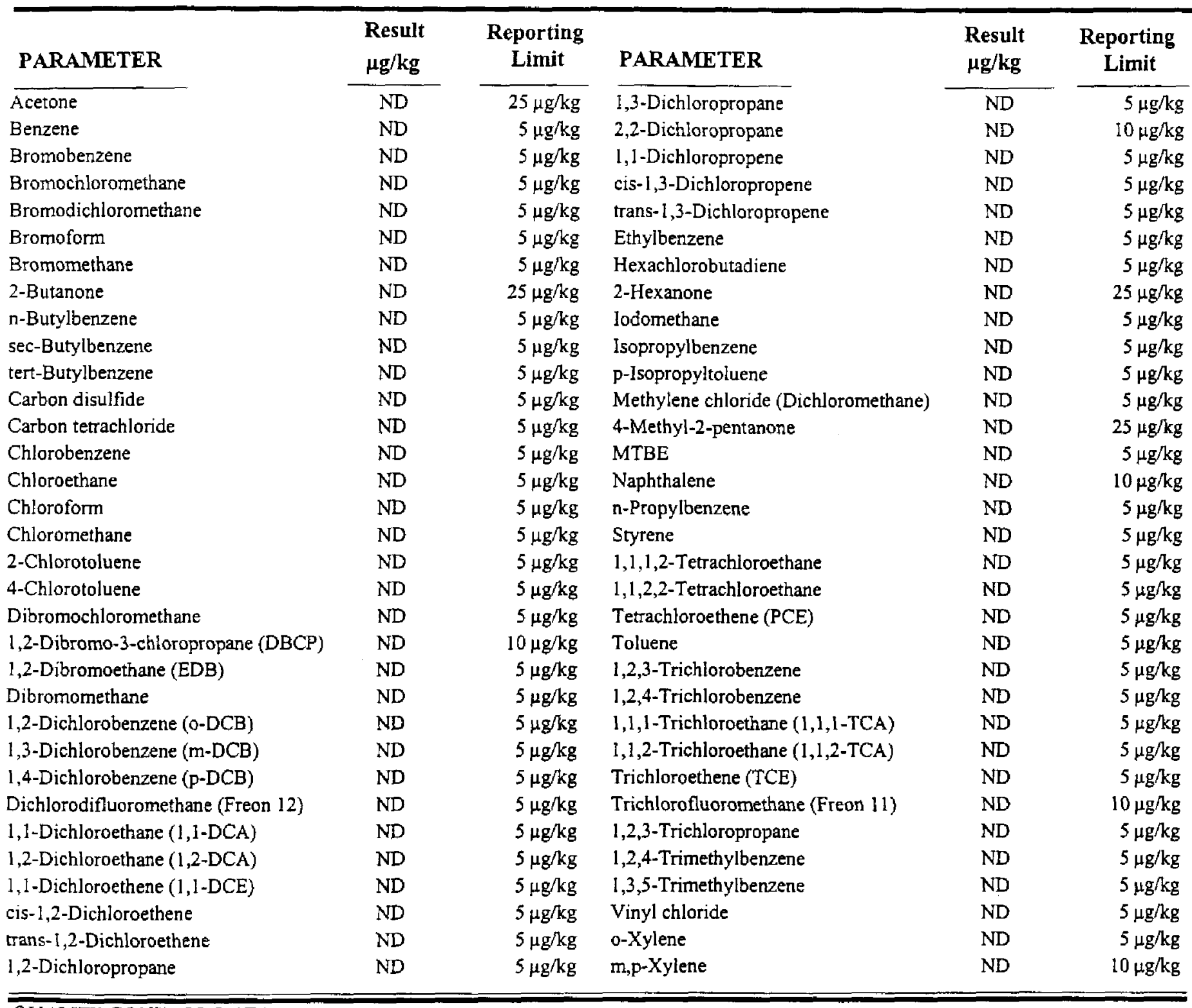

QUALITY CONTROL DATA:

\section{Surrogate}

4-Bromofiuorobenzene

Dibromofluoromethane

Toluene-d8
$\%$ Recovery

103

103

105
Acceptable Range

$74-121$

$80-120$

$81-117$

ND - Not Detected

This report shall not be reproduced except in full, without the written approval of the laboratory. 
NEL LABORATORIES

\begin{tabular}{llll}
\hline CLIENT: & Bechtel Nevada & CLIENT ID: & $\mathbf{0 3 2 2 1} \div-\mathbf{- 0} \mathbf{v}$ \\
PROJECT ID: & V1358 & DATE SAMPLED: $11 / 27 / 01$ \\
PROJECT & 30033 & 303 & NEL SAMPLE ID: L0111248-03
\end{tabular}

TEST: $\quad$ Total Extractable Petroleum Hydrocarbons Fuel Finger Print by EPA Method 8015M, July 1992

METHOD: EPA 8015M

MATRIX: Solid

ANALYST: $\quad$ PXC-Division

DILUTION: 1

EXTRACTED: $\quad 11 / 30 / 01$

ANALYZED: $\quad 12 / 3 / 01$

\begin{tabular}{lcc}
\hline PARAMETER & Result & Reporting \\
\cline { 2 - 3 } & $\mathrm{ND}$ & Limit \\
\hline Gasoline Range $(\mathrm{C} 8-\mathrm{Cl} 2)$ & $\mathrm{ND}$ & $10 . \mathrm{mg} / \mathrm{kg}$ \\
Diesel Range $(\mathrm{C} 12-\mathrm{C} 22)$ & $\mathrm{ND}$ & $10 . \mathrm{mg} / \mathrm{kg}$ \\
Oil Range $(\mathrm{C} 22-\mathrm{C} 34)$ & $\mathrm{ND}$ & $50 \mathrm{mg} / \mathrm{kg}$ \\
Total & & $10 . \mathrm{mg} / \mathrm{kg}$ \\
\hline \hline
\end{tabular}

QUALITY CONTROL DATA:

Surrogate

$\%$ Recovery

Octacosane

73

Acceptable Range

$54-130$

ND - Not Detected

This report shall not be reproduced except in full, without the written approval of the laboratory. 
NEL LABORATORIES

\begin{tabular}{llll}
\hline CLIENT: & Bechtel Nevada & CLIENT D: & $\mathbf{0 3 2 2 0 : - 6 - V}$ \\
PROJECT ID: & V1358 & DATE SAMPLED: $11 / 27 / 01$ \\
PROJECT \#: & 30033 & NEL SAMPLE ID: L0111248-04
\end{tabular}

TEST: $\quad$ Total Extractable Petroleum Hydrocarbons Fuel Finger Print by EPA Method 8015M, July 1992

METHOD: $\quad$ EPA $8015 \mathrm{M}$

MATRIX: Solid

ANALYST: $\quad$ PXC - Division

DILUTION: 1

EXTRACTED: $\quad 11 / 30 / 01$

ANALYZED: $\quad 12 / 3 / 01$

Reporting

PARAMETER

Gasoline Range (C $8-\mathrm{C} 12)$

Diesel Range (C12-C22)

Oil Range (C22-C34)

\section{Result}

ND

ND

ND

ND

QUALITY CONTROL DATA:

Surrogate

\% Recovery

Octacosane

69

Octacosane
Limit

10. $\mathrm{mg} / \mathrm{kg}$

10. $\mathrm{mg} / \mathrm{kg}$

50. $\mathrm{mg} / \mathrm{kg}$

10. $\mathrm{mg} / \mathrm{kg}$

ND - Not Detected

This report shall not be reproduced except in full, without the written approval of the laboratory. 
NEL LABORATORIES

\begin{tabular}{llll}
\hline CLIENT: & Bechtel Nevada & CLIENT D: & Meth jỏ Blank \\
PROJECT ID: & V1358 & DATE SAMPLED: & NA \\
PROJECT \#: & 30033 & NEL SAMPLE ID: & 011130TPHS-FP-BLK
\end{tabular}

TEST:

METHOD:

Total Extractable Petroleum Hydrocarbons Fuel Finger Print by EPA Method 8015M, July 1992

MATRIX: EPA $8015 \mathrm{M}$

Solid

ANALYST: $\quad$ PXC - Division

EXTRACTED: $\quad 11 / 30 / 01$

ANALYZED: $\quad 12 / 3 / 01$

\section{PARAMETER}

Gasoline Range (C8-C12)

Diesel Range (C12-C22)

Oil Range (C22-C34)

Total

\begin{tabular}{l} 
Result \\
\hline ND \\
ND \\
ND \\
ND
\end{tabular}

Reporting

QUALITY CONTROL DATA:

Surrogate

$\%$ Recovery

Octacosane

93

Acceptable Range

$54-130$

ND - Not Detected

This report shall not be reproduced except in full, without the written approval of the laboratory. 
CLIENT: Bechtel Nevada

PROJECT ID: V1358

PROJECT \#: 30033

TEST: $\quad$ Semi-Volatile Organic Compounds by EPA 8270C, Dec. 1996

MATRIX: Solid

\begin{tabular}{|c|c|c|c|c|c|c|c|}
\hline PARAMETER & NEL Sample ID & $\frac{\text { Spike }}{\text { Amount }}$ & $\frac{\text { Spike }}{\underline{\text { Result }}}$ & $\underline{\text { Percent }}$ & & $\frac{\text { Acceptable }}{\text { Range }}$ & $\underline{\text { RPD }}$ \\
\hline Acenaphthene & $011129-8270$ S-LCS & 1667 & 1444 & 87 & & $31-137$ & \\
\hline Acenaphthene & $011129-8270$ S-LCSD & 1667 & 1213 & 73 & & $31-137$ & 17.4 \\
\hline Acenaphthene & L0111248-04-MS & 1667 & 1087 & 65 & & $31-137$ & \\
\hline Acenaphthene & L0111248-04-MSD & 1667 & 1173 & 70 & & $31-137$ & 7.6 \\
\hline Acenaphthylene & $011129-8270$ S-LCS & 1667 & 1455 & 87 & & $50-120$ & \\
\hline Acenaphthylene & $011129-8270$ S-LCSD & 1667 & 1210 & 73 & & $50-120$ & 18.4 \\
\hline Acenaphthylene & L0111248-04-MS & 1667 & 1101 & 66 & & $50-120$ & \\
\hline Acenaphthylene & L0111248-04-MSD & 1667 & 1161 & 70 & & $50-120$ & 5.3 \\
\hline Aniline & $011129-8270$ S-LCS & 1667 & 1507 & 90 & & $11-77$ & \\
\hline Aniline & $011129-8270$ S-LCSD & 1667 & 1310 & 79 & & $11-77$ & 14. \\
\hline Aniline & L0111248-04-MS & 1667 & 647 & 39 & & $11-77$ & \\
\hline Aniline & L0111248-04-MSD & 1667 & 895 & 54 & R5 & $11-77$ & 32.2 \\
\hline Anthracene & $011129-8270 S-L C S$ & 1667 & 1532 & 92 & & $50-120$ & \\
\hline Anthracene & $011129-8270 S-L C S D$ & 1667 & 1355 & 81 & & $50-120$ & 12.3 \\
\hline Anthracene & L0111248-04-MS & 1667 & 1339 & 80 & & $50-120$ & \\
\hline Anthracene & L0111248-04-MSD & 1667 & 1357 & 81 & & $50-120$ & 1.3 \\
\hline Azobenzene & $011129-8270 S-L C S$ & 1667 & 1379 & 83 & & $22-123$ & \\
\hline Azobenzene & $011129-8270 S-L C S D$ & 1667 & 1210 & 73 & & $22-123$ & 13.1 \\
\hline Azobenzene & L0111248-04-MS & 1667 & 1192 & 72 & & $22-123$ & \\
\hline Azobenzene & L0111248-04-MSD & 1667 & 1197 & 72 & & $22=123$ & 0.4 \\
\hline Benzo (a) Anthracene & $011129-8270 \mathrm{~S}-\mathrm{LCS}$ & 1667 & 1581 & 95 & & $50-120$ & \\
\hline Benzo (a) Anthracene & $011129-8270 S-L C S D$ & 1667 & 1292 & 78 & & $50-120$ & 20.1 \\
\hline Benzo (a) Anthracene & L0111248-04-MS & 1667 & 1267 & 76 & & $50-120$ & \\
\hline Benzo (a) Anthracene & L0111248-04-MSD & 1667 & 1307 & 78 & & $50-120$ & 3.1 \\
\hline Benzoic Acid & $011129-8270$ S-LCS & 1667 & 267 & 16 & $\mathrm{~J}$ & $50-120$ & \\
\hline Benzoic Acid & $011129-8270$ S-LCSD & 1667 & 267 & 16 & $\mathrm{~J}$ & $50-120$ & 0. \\
\hline Benzoic Acid & L0111248-04-MS & 1667 & 591 & 35 & J & $50-120$ & \\
\hline Benzoic Acid & L0111248-04-MSD & 1667 & $833^{\circ}$ & 50 & J & $50-120$ & 34. \\
\hline Benzo $(g, h, i)$ perylene & $011129-8270$ S-LCS & 1667 & 1397 & 84 & & $50-120$ & \\
\hline Benzo $(g, h, i)$ perylene & 011129-8270S-LCSD & 1667 & 1217 & 73 & & $50-120$ & 13.8 \\
\hline Benzo $(\mathrm{g}, \mathrm{h}, \mathrm{i})$ perylene & L01 $11248-04-M S$ & 1667 & 1332 & 80 & & $50-120$ & \\
\hline Benzo $(g, h, i)$ perylene & L0111248-04-MSD & 1667 & 1400 & 84 & & $50-120$ & 5. \\
\hline Benzo (a) pyrene & $011129-8270 S-L C S$ & 1667 & 1554 & 93 & & $50-130$ & \\
\hline 3enzo (a) pyrene & $011129-8270 S-L C S D$ & 1667 & 1381 & 83 & & $50-130$ & 11.8 \\
\hline Benzo (a) pyrene & L0111248-04-MS & 1667 & 1284 & 77 & & $50-130$ & \\
\hline Senzo (a) pyrene & L0111248-04-MSD & 1667 & 1384 & 83 & & $50-130$ & 7.5 \\
\hline 3enzyl alcohol & $011129-8270$ S-LCS & 1667 & 1405 & 84 & & $0-116$ & \\
\hline Senzyl alcohol & 011129-8270S-LCSD & 1667 & 1151 & 69 & & $0-116$ & 19.9 \\
\hline
\end{tabular}

ND - Not Detected

This report shall not be reproduced except in full, without the written approval of the laboratory. 
CLIENT: Bechtel Nevada

PROJECT ID: V1358

PROJECT \#: 30033

TEST: $\quad$ Semi-Volatile Organic Compounds by EPA 8270C, Dec. 1996

MATRIX: Solid

\begin{tabular}{|c|c|c|c|c|c|c|}
\hline PARAMETER & NEL Sample ID & $\frac{\text { Spike }}{\text { Amount }}$ & $\frac{\text { Spike }}{\text { Result }}$ & $\frac{\text { Percent }}{\text { Recovery }}$ & $\frac{\text { Acceptable }}{\text { Range }}$ & $\underline{\text { RPD }}$ \\
\hline Benzyl alcohol & L0111248-04-MS & 1667 & 1058 & 63 & $0-116$ & \\
\hline Benzyl alcohol & L0111248-04-MSD & 1667 & 1082 & 65 & $0-116$ & 2.2 \\
\hline bis (2-Chloroethyl) ether & $011129-8270$ S-LCS & 1667 & 1329 & 80 & $15-107$ & \\
\hline jis (2-Chloroethyl) ether & 011129-8270S-LCSD & 1667 & 1075 & 64 & $15-107$ & 21.1 \\
\hline bis (2-Chloroethyl) ether & L0111248-04-MS & 1667 & 946 & 57 & $15-107$ & \\
\hline bis (2-Chloroethyl) ether & L0111248-04-MSD & 1667 & 959 & 58 & $15-107$ & 1.4 \\
\hline bis (2-Chloroethoxy) methane & $011129-8270$ S-LCS & 1667 & 1360 & 82 & $26-97$ & \\
\hline bis (2-Chloroethoxy) methane & $011129-8270$ S-LCSD & 1667 & 1121 & 67 & $26-97$ & 19.3 \\
\hline bis (2-Chloroethoxy) methane & L0111248-04-MS & 1667 & 997 & 60 & $26-97$ & \\
\hline bis (2-Chloroethoxy) methane & L0111248-04-MSD & 1667 & 1071 & 64 & $26-97$ & 7.2 \\
\hline bis (2-chloroisopropyl) ether & $011129-8270$ S-LCS & 1667 & 1381 & 83 & $50-120$ & \\
\hline bis (2-chloroisopropyl) ether & $011129-8270$ S-LCSD & 1667 & 1138 & 68 & $50-120$ & 19.3 \\
\hline ois (2-chloroisopropyl) ether & L0111248-04-MS & 1667 & 969 & 58 & $50-120$ & \\
\hline jis (2-chloroisopropyl) ether & L0111248-04-MSD & 1667 & 1000 & 60 & $50-120$ & 3.1 \\
\hline jis (2-Ethylhexyl)phthalate & $011129-8270$ S-LCS & 1667 & 1568 & 94 & $50-120$ & \\
\hline jis (2-Ethylhexyl)phthalate & 011129-8270S-LCSD & 1667 & 1339 & 80 & $50-120$ & 15.8 \\
\hline jis (2-Ethylhexyl)phthalate & L0111248-04-MS & 1667 & 1289 & 77 & $50-120$ & \\
\hline jis (2-Ethylhexyl)phthalate & L01 I 1248-04-MSD & 1667 & 1366 & 82 & $50-120$ & 5.8 \\
\hline 3utyl benzyl phthalate & $011129-8270 S-L C S$ & 1667 & 1556 & 93 & $50-120$ & \\
\hline 3utyl benzyl phthalate & 011129-8270S-LCSD & 1667 & 1298 & 78 & $50-120$ & 18.1 \\
\hline 3utyl benzyl phthalate & L0111248-04-MS & 1667 & 1296 & 78 & $50-120$ & \\
\hline 3utyl benzyl phthalate & L0111248-04-MSD & 1667 & 1314 & 79 & $50-120$ & 1.4 \\
\hline t-Bromophenyl phenyl ether & $011129-8270 \mathrm{~S}-\mathrm{LCS}$ & 1667 & 1573 & 94 & $50-120$ & \\
\hline f-Bromophenyl phenyl ether & $011129-8270$ S-LCSD & 1667 & 1353 & 81 & $50-120$ & 15. \\
\hline 1-Bromophenyl phenyl ether & L0111248-04-MS & 1667 & 1340 & 80 & $50-120$ & \\
\hline t-Bromophenyl phenyl ether & L0111248-04-MSD & 1667 & 1358 & 81 & $50-120$ & 1.3 \\
\hline Zarbazole & $011129-8270 S-L C S$ & 1667 & 1586 & 95 & $50-120$ & \\
\hline Zarbazole & 011129-8270S-LCSD & 1667 & 1371 & 82 & $50-120$ & 14.5 \\
\hline Zarbazole & L0111248-04-MS & 1667 & 1322 & 79 & $50-120$ & \\
\hline Zarbazole & L0111248-04-MSD & 1667 & 1350 & 81 & $50-120$ & 2.1 \\
\hline i-Chloroaniline & $011129-8270 S-L C S$ & 1667 & 1291 & 77 & $1-68$ & \\
\hline -Chloroaniline & $011129-8270 S-L C S D$ & 1667 & 1120 & 67 & $1-68$ & 0. \\
\hline -Chloroaniline & L0111248-04-MS & 1667 & 619 & 37 & $1-68$ & \\
\hline -Chloroaniline & L0111248-04-MSD & 1667 & 864 & $52 \quad \mathrm{R} 5$ & $1-68$ & 33. \\
\hline -Chloro-3-methyl phenol & 011129-8270S-LCS & 1667 & 1459 & 88 & $26-103$ & \\
\hline -Chloro-3-methyl phenol & 011129-8270S-LCSD & 1667 & 1244 & 75 & $26-103$ & 15.9 \\
\hline -Chloro-3-methyl phenol & L0111248-04-MS & 1667 & 1269 & 76 & $26-103$ & \\
\hline -Chloro-3-methyl phenol & L0111248-04-MSD & 1667 & 1282 & 77 & $26-103$ & 1. \\
\hline
\end{tabular}

VD - Not Detected

This report shall not be reproduced except in full, without the written approval of the laboratory. 


\begin{tabular}{ll}
\hline CLIENT: & Bechtel Nevada \\
PROJECT ID: & V1358 \\
PROJECT \#: & 30033 \\
TEST: & Semi-Volatile Organic Compounds by EPA 8270C, Dec. 1996 \\
MATRIX: & Solid
\end{tabular}

\begin{tabular}{|c|c|c|c|c|c|c|}
\hline PARAMETER & NEL Sample ID & $\frac{\text { Spike }}{\text { Amount }}$ & $\frac{\text { Spike }}{\text { Result }}$ & $\frac{\text { Percent }}{\text { Recovery }}$ & $\frac{\text { Acceptable }}{\text { Range }}$ & $\underline{\text { RPD }}$ \\
\hline 2-Chioronaphthalene & $011129-8270$ S-LCS & 1667 & 1399 & 84 & $50-120$ & \\
\hline 2-Chloronaphthalene & $011129-8270 S-L C S D$ & 1667 & 1133 & 68 & $50-120$ & 21. \\
\hline 2-Chloronaphthalene & L0111248-04-MS & 1667 & 1046 & 63 & $50-120$ & \\
\hline 2-Chloronaphthalene & L0111248-04-MSD & 1667 & 1095 & 66 & $50-120$ & 4.6 \\
\hline 2-Chlorophenol & $011129-8270 S-L C S$ & 1667 & 1340 & 80 & $25-102$ & \\
\hline 2-Chlorophenol & 011129-8270S-LCSD & 1667 & 1135 & 68 & $25-102$ & 16.6 \\
\hline 2-Chlorophenol & L0111248-04-MS & 1667 & 1014 & 61 & $25-102$ & \\
\hline 2-Chlorophenol & L0111248-04-MSD & 1667 & 1033 & 62 & $25-102$ & 1.9 \\
\hline 4-Chlorophenyl phenyl ether & $011129-8270 S-L C S$ & 1667 & 1442 & 87 & $50-120$ & \\
\hline 4-Chlorophenyl phenyl ether & $011129-8270 \mathrm{~S}-\mathrm{LCSD}$ & 1667 & 1242 & 75 & $50-120$ & 14.9 \\
\hline 4-Chlorophenyl phenyl ether & L0111248-04-MS & 1667 & 1185 & 71 & $50-120$ & \\
\hline 4-Chlorophenyl phenyl ether & L0111248-04-MSD & 1667 & 1200 & 72 & $50-120$ & 1.3 \\
\hline Chrysene & $011129-8270 S-L C S$ & 1667 & 1511 & 91 & $50-120$ & \\
\hline Chrysene & $011129-8270 \mathrm{~S}-\mathrm{LCSD}$ & 1667 & 1317 & 79 & $50-120$ & 13.7 \\
\hline Chrysene & L0111248-04-MS & 1667 & 1221 & 73 & $50-120$ & \\
\hline Chrysene & L0111248-04-MSD & 1667 & 1339 & 80 & $50-120$ & 9.2 \\
\hline Dibenz $(\mathrm{a}, \mathrm{h})$ anthracene & $011129-8270 S-L C S$ & 1667 & 1492 & 90 & $50-120$ & \\
\hline Dibenz $(a, h)$ anthracene & $011129-8270$ S-LCSD & 1667 & 1293 & 78 & $50-120$ & 14.3 \\
\hline Dibenz $(\mathrm{a}, \mathrm{h})$ anthracene & L0111248-04-MS & 1667 & 1367 & 82 & $50-120$ & \\
\hline Dibenz $(\mathrm{a}, \mathrm{h})$ anthracene & L0111248-04-MSD & 1667 & 1446 & 87 & $50-120$ & 5.6 \\
\hline Dibenzofuran & $011129-8270 S-L C S$ & 1667 & 1386 & 83 & $50-120$ & \\
\hline Dibenzofuran & $011129-8270 S-L C S D$ & 1667 & 1173 & 70 & $50-120$ & 16.6 \\
\hline Dibenzofuran & L0111248-04-MS & 1667 & 1122 & 67 & $50-120$ & \\
\hline Dibenzofuran & L0111248-04-MSD & 1667 & 1212 & 73 & $50-120$ & 7.7 \\
\hline Di-n-butyl phthalate & $011129-8270 \mathrm{~S}-\mathrm{LCS}$ & 1667 & 1647 & 99 & $50-120$ & \\
\hline Di-n-butyl phthalate & 011129-8270S-LCSD & 1667 & 1366 & 82 & $50-120$ & 18.7 \\
\hline Di-n-butyl phthalate & L0111248-04-MS & 1667 & 1371 & 82 & $50-120$ & \\
\hline Di-n-butyl phthalate & L0111248-04-MSD & 1667 & 1518 & 91 & $50-120$ & 10.2 \\
\hline 1,2-Dichlorobenzene (o-DCB) & $011129-8270$ S-LCS & 1667 & 1391 & 83 & $42-93$ & \\
\hline 1,2-Dichlorobenzene (o-DCB) & $011129-8270 S-L C S D$ & 1667 & 1097 & 66 & $42-93$ & 23.6 \\
\hline 1,2-Dichlorobenzene (o-DCB) & L0111248-04-MS & 1667 & 979 & 59 & $42-93$ & \\
\hline 1,2-Dichlorobenzene (o-DCB) & L0111248-04-MSD & 1667 & 989 & 59 & $42-93$ & 1. \\
\hline 1,3-Dichlorobenzene (m-DCB) & $011129-8270$ S-LCS & 1667 & 1305 & 78 & $41-89$ & \\
\hline 1,3-Dichlorobenzene (m-DCB) & 011129-8270S-LCSD & 1667 & 1054 & 63 & $41-89$ & 21.3 \\
\hline 1,3-Dichlorobenzene (m-DCB) & L0111248-04-MS & 1667 & 925 & 55 & $41 \cdot 89$ & \\
\hline 1,3-Dichlorobenzene (m-DCB) & L0111248-04-MSD & 1667 & 967 & 58 & $41-89$ & 4.4 \\
\hline 1,4-Dichlorobenzene (p-DCB) & $011129-8270 S-L C S$ & 1667 & 1312 & 79 & $42-93$ & \\
\hline 1,4-Dichlorobenzene (p-DCB) & 011129-8270S-LCSD & 1667 & 1056 & 63 & $42-93$ & 21.6 \\
\hline
\end{tabular}


NEL LABORATORIES

\section{CLIENT: Bechtel Nevada}

PROJECT ID: V1358

PROJECT \#: 30033

TEST:

Semi-Volatile Organic Compounds by EPA 8270C, Dec. 1996

MATRIX:

Solid

\section{PARAMETER}

1,4-Dichlorobenzene ( $\mathrm{p}$-DCB)

1,4-Dichlorobenzene ( $\mathrm{p}-\mathrm{DCB})$

2,4-Dichlorophenol

2,4-Dichlorophenol

2,4-Dichlorophenol

2,4-Dichlorophenol

3,3'-Dichlorobenzidine

3,3'-Dichlorobenzidine

3,3'-Dichlorobenzidine

3,3'-Dichlorobenzidine

Diethyl Phthalate

Diethyl Phthalate

Diethyl Phthalate

Diethyl Phthalate

2,4-Dimethylphenol

2,4-Dimethylphenol

2,4-Dimethyiphenol

2,4-Dimethylphenol

Dimethyl phthalate

Dimethyl phthalate

Dimethyl phthalate

Dimethyl phthalate

4,6-Dinitro-2-methyl phenol

4,6-Dinitro-2-methyl phenol

4,6-Dinitro-2-methyl phenol

7,6-Dinitro-2-methyl phenol

2,4-Dinitrotoluene (DNT)

2,4-Dinitrotoluene (DNT)

2,4-Dinitrotoluene (DNT)

2,4-Dinitrotoluene (DNT)

2,6-Dinitrotoluene (DNT)

2,6-Dinitrotoluene (DNT)

3,6 -Dinitrotoluene (DNT)

2,6-Dinitrotoluene (DNT)

?,4-Dinitrophenol

?,4-Dinitrophenol

?,4-Dinitrophenol

?,4-Dinitrophenol

\section{NEL Sample ID}

L0111248-04-MS

L0111248-04-MSD

$011129-8270$ S-LCS

011129-8270S-LCSD

L0111248-04-MS

L0111248-04-MSD

011129-8270S-LCS

011129-8270S-LCSD

L0111248-04-MS

L0111248-04-MSD

011129-8270S-LCS

$011129-8270$ S-LCSD

L0111248-04-MS

L0111248-04-MSD

011129-8270S-LCS

$011129-8270 S-L C S D$

L011 1248-04-MS

L0111248-04-MSD

011129-8270S-LCS

$011129-8270 S-L C S D$

L0111248-04-MS

L0111248-04-MSD

011129-8270S-LCS

$011129-8270 S-L C S D$

L011 1248-04-MS

L0111248-04-MSD

011129-8270S-LCS

011129-8270S-LCSD

L011 1248-04-MS

L0111248-04-MSD

011129-8270S-LCS

011129-8270S-LCSD

L0111248-04-MS

L0111248-04-MSD

011129-8270S-LCS

$011129-8270$ S-LCSD

L0111248-04-MS

L01 1 1248-04-MSD

\section{Spike}

Amount

1667

1667

1667

1667

1667

1667

1667

1667

1667

1667

1667

1667

1667

1667

1667

1667

1667

1667

1667

1667

1667

1667

1667

1667

1667

1667

1667

1667

1667

1667

1667

1667

1667

1667

1667

1667

1667

1667

\section{Spike}

Result

931

933

1472

1165

1205

1279

1427

1208

974

985

1543

1340

1275

1272

1409

1191

1023

1118

1497

1316

1217

1290

875

831

1342

1422

1476

1261

1224

1236

1470

1257

1184

1223

625

534

1132

1185

\begin{tabular}{c}
$\frac{\text { Percent }}{\text { Recovery }}$ \\
\hline 56 \\
56 \\
88 \\
70
\end{tabular}

Acceptable

Range RPD

$42-93$

$42-93$

0.2

$50-120$

$50-120$

23.3

$50-120$

$50-120$

6.

$11-85$

$11-85$

$11-85$

$11-85$

$50-120$

$50-120$

$50-120$

$50-120$

$50-120$

$50-120$

$50-120$

$50-120$

$50-120$

$50-120$

$50-120$

$50-120$

$20-120$

$20-120$

$20-120$

$20-120$

$50-111$

$50-111$

$50-111$

$50-111$

$50-120$

$50-120$

15.6

$50-120$

$50-120$

3.2

$20-120$

$20-120$

15.7

$20-120$

$20-120$

VD - Not Detected

This report shall not be reproduced except in full, without the written approval of the laboratory. 
CIIENT:

Bechtel Nevada

PROJECT ID: V1358

PROJECT \#: $\quad 30033$

TEST: $\quad$ Semi-Volatile Organic Compounds by EPA 8270C, Dec. 1996

MATRIX: Solid

\begin{tabular}{|c|c|c|c|c|c|c|c|}
\hline PARAMETER & NEL Sample ID & $\underline{\text { Spike }}$ & $\frac{\text { Spike }}{\underline{\text { Result }}}$ & $\frac{\text { Percent }}{\text { Recovery }}$ & & $\frac{\text { Acceptable }}{\text { Range }}$ & $\underline{\mathbf{R P D}}$ \\
\hline Di-n-octyl phthalate & $011129-8270 \mathrm{~S}-\mathrm{LCS}$ & 1667 & 1624 & 97 & & $50 \cdot 120$ & \\
\hline Di-n-octyl phthaiate & 011129-8270\$-LCSD & 1667 & 1413 & 85 & & $50-120$ & 13.9 \\
\hline Di-n-octyl phthalate & L0111248-04-MS & 1667 & 1360 & 82 & & $50-120$ & \\
\hline Di-n-octyl phthalate & L0111248-04-MSD & 1667 & 1405 & 84 & & $50-120$ & 3.3 \\
\hline Fluoranthene & $011129-8270$ S-LCS & 1667 & 1540 & 92 & & $50-120$ & \\
\hline Fluorene & $011129-8270$ S-LCS & 1667 & 1474 & 88 & & $50-120$ & \\
\hline Hexachlorobenzene & $011129-8270 \mathrm{~S}-\mathrm{LCS}$ & 1667 & 1565 & 94 & & $50-120$ & \\
\hline Hexachlorobenzene & $011129-8270 S-L C S D$ & 1667 & 1391 & 83 & & $50-120$ & 11.8 \\
\hline Hexachlorobenzene & L0111248-04-MS & 1667 & 1349 & 81 & & $50-120$ & \\
\hline Hexachlorobenzene & L0111248-04-MSD & 1667 & 1398 & 84 & & $50-120$ & 3.6 \\
\hline Hexachlorobutadiene & $011129-8270 \mathrm{~S}-\mathrm{LCS}$ & 1667 & 1450 & 87 & & $30-120$ & \\
\hline Hexachlorobutadiene & 011129-8270S-LCSD & 1667 & 1213 & 73 & & $30-120$ & 17.8 \\
\hline Hexachlorobutadiene & L0111248-04-MS & 1667 & 1037 & 62 & & $30-120$ & \\
\hline Hexachlorobutadiene & L0111248-04-MSD & 1667 & 1118 & 67 & & $30-120$ & 7.5 \\
\hline Hexachlorocyclopentadiene & $011129-8270$ S-LCS & 1667 & 972 & 58 & & $20-120$ & \\
\hline Hexachlorocyclopentadiene & $011129-8270 \mathrm{~S}-\mathrm{LCSD}$ & 1667 & 773 & 46 & & $20-120$ & 22.8 \\
\hline Hexachlorocyclopentadiene & L0111248-04-MS & 1667 & 61.71 & 4 & M2 & $20-120$ & \\
\hline Hexachlorocyclopentadiene & L0111248-04-MSD & 1667 & 548 & 33 & R5 & $20-120$ & 0. \\
\hline Hexachloroethane & $011129-8270 \mathrm{~S}-\mathrm{LCS}$ & 1667 & 1171 & 70 & & $30-120$ & \\
\hline Hexachloroethane & $011129-8270$ S-LCSD & 1667 & 985 & 59 & & $30-120$ & 17.3 \\
\hline Hexachloroethane & L0111248-04-MS & 1667 & 851 & 51 & & $30-120$ & \\
\hline Hexachloroethane & L0111248-04-MSD & 1667 & 894 & 54 & & $30-120$ & 4.9 \\
\hline Indeno $(1,2,3-c, d)$ pyrene & 011129-8270S-LCS & 1667 & 1487 & 89 & & $50-120$ & \\
\hline Indeno $(1,2,3-c, d)$ pyrene & $011129-8270$ S-LCSD & 1667 & 1306 & 78 & & $50-120$ & 13. \\
\hline Indeno $(1,2,3-c, d)$ pyrene & L0111248-04-MS & 1667 & 1375 & 82 & & $50-120$ & \\
\hline Indeno $(1,2,3-c, d)$ pyrene & L0111248-04-MSD & 1667 & 1480 & 89 & & $50-120$ & 7.4 \\
\hline isophorone & $011129-8270 \mathrm{~S}-\mathrm{LCS}$ & 1667 & 1302 & 78 & & $24-99$ & \\
\hline Sophorone & $011129-8270 \mathrm{~S}-\mathrm{LCSD}$ & 1667 & 1050 & 63 & & $24-99$ & 21.4 \\
\hline !sophorone & L0111248-04-MS & 1667 & 975 & 58 & & $24-99$ & \\
\hline sophorone & L0111248-04-MSD & 1667 & 1025 & 61 & & $24-99$ & 5. \\
\hline 2-Methylnaphthalene & $011129-8270$ S-LCS & 1667 & 1346 & 81 & & $50-120$ & \\
\hline 2-Methylnaphthalene & $011129-8270$ S-LCSD & 1667 & 1106 & 66 & & $50-120$ & 19.6 \\
\hline 2-Methylnaphthalene & L0111248-04-MS & 1667 & 1035 & 62 & & $50-120$ & \\
\hline 2-Methylnaphthalene & L0111248-04-MSD & 1667 & 1123 & 67 & & $50-120$ & 8.2 \\
\hline 2-Methylphenol & $011129.8270 \mathrm{~S}-\mathrm{LCS}$ & 1667 & 1394 & 84 & & $14-108$ & \\
\hline 2-Methylphenol & $011129-8270 \mathrm{~S}-\mathrm{LCSD}$ & 1667 & 1125 & 67 & & $14-108$ & 21.4 \\
\hline 2-Methylphenol & L0111248-04-MS & 1667 & 1019 & 61 & & $14-108$ & \\
\hline 2-Methylphenol & L0111248-04-MSD & 1667 & 1014 & 61 & & $14-108$ & 0.5 \\
\hline
\end{tabular}

ND - Not Detected

This report shall not be reproduced except in full, without the written approval of the laboratory. 
CLIENT: Bechtel Nevada

PROJECT ID: V1358

PROJECT \#: 30033

TEST: $\quad$ Semi-Volatile Organic Compounds by EPA 8270C, Dec. 1996

MATRIX: Solid

\begin{tabular}{|c|c|c|c|c|c|c|}
\hline PARAMETER & NEL Sample ID & $\frac{\text { Spike }}{\text { Amount }}$ & $\underline{\text { Spike }}$ & $\frac{\text { Percent }}{\text { Recovery }}$ & $\frac{\text { Acceptable }}{\underline{\text { Range }}}$ & $\underline{\mathbf{R P D}}$ \\
\hline Naphthalene & $\overline{011129-8270 \mathrm{~S}-\mathrm{LCS}}$ & 1667 & 1363 & 82 & $50-120$ & \\
\hline Naphthalene & $011129-8270 \mathrm{~S}-\mathrm{LCSD}$ & 1667 & 1138 & 68 & $50-120$ & 18. \\
\hline Naphthaiene & L0111248-04-MS & 1667 & 1018 & 61 & $50-120$ & \\
\hline Naphthalene & L0111248-04-MSD & 1667 & 1049 & 63 & $50-120$ & 3. \\
\hline 2-Nitroaniline & $011129-8270$ S-LCS & 1667 & 1476 & 89 & $30-120$ & \\
\hline 2-Nitroaniline & $011129-8270 S-L C S D$ & 1667 & 1289 & 77 & $30-120$ & 13.5 \\
\hline 2-Nitroaniline & L0111248-04-MS & 1667 & 1216 & 73 & $30-120$ & \\
\hline 2-Nitroaniline & L0111248-04-MSD & 1667 & 1244 & 75 & $30-120$ & 2.3 \\
\hline 3-Nitroaniline & $011129-8270$ S-LCS & 1667 & 1388 & 83 & $30-120$ & \\
\hline 3-Nitroaniline & $011129-8270 \mathrm{~S}-\mathrm{LCSD}$ & 1667 & 1242 & 75 & $30-120$ & 11.1 \\
\hline 3-Nitroaniline & L0111248-04-MS & 1667 & 928 & 56 & $30-120$ & \\
\hline 3-Nitroaniline & L0111248-04-MSD & 1667 & 1044 & 63 & $30-120$ & 11.8 \\
\hline 4-Nitroaniline & $011129-8270 \mathrm{~S}-\mathrm{LCS}$ & 1667 & 1487 & 89 & $30-120$ & \\
\hline 4-Nitroaniline & $011129-8270 \mathrm{~S}-\mathrm{LCSD}$ & 1667 & 1299 & 78 & $30-120$ & 13.5 \\
\hline 4-Nitroaniline & L0111248-04-MS & 1667 & 1411 & 85 & $30-120$ & \\
\hline 4-Nitroaniline & L0111248-04-MSD & 1667 & 1417 & 85 & $30-120$ & 0.4 \\
\hline Nitrobenzene & $011129-8270 S-L C S$ & 1667 & 1383 & 83 & $23-103$ & \\
\hline Nitrobenzene & 011129-8270S-LCSD & 1667 & 1123 & 67 & $23-103$ & 20.8 \\
\hline Nitrobenzene & L0111248-04-MS & 1667 & 1022 & 61 & $23-103$ & \\
\hline Nitrobenzene & L0111248-04-MSD & 1667 & 1087 & 65 & $23-103$ & 6.2 \\
\hline 2-Nitrophenol & $011129-8270 S-\operatorname{LCS}$ & 1667 & 1672 & 100 & $42-86$ & \\
\hline 2-Nitrophenol & 011129-8270S-LCSD & 1667 & 1253 & 75 & $42-86$ & 0. \\
\hline 2-Nitrophenol & L0111248-04-MS & 1667 & 1222 & 73 & $42-86$ & \\
\hline 2-Nitrophenol & L0111248-04-MSD & 1667 & 1255 & 75 & $42-86$ & 2.7 \\
\hline 4-Nitrophenol & $011129-8270 S-L C S$ & 1667 & 1487 & 89 & $22-151$ & \\
\hline 4-Nitrophenol & $011129-8270 \mathrm{~S}-\mathrm{LCSD}$ & 1667 & 1299 & 78 & $22-151$ & 13.5 \\
\hline +-Nitrophenol & L0111248-04-MS & 1667 & 1411 & 85 & $22-151$ & \\
\hline 1-Nitrophenol & L0111248-04-MSD & 1667 & 1417 & 85 & $22-151$ & 0.4 \\
\hline$\checkmark-N i t r o s o d i m e t h y l a m i n e$ & $011129-8270$ S-LCS & 1667 & 1471 & 88 & $9-97$ & \\
\hline V-Nitrosodimethylamine & 011129-8270S-LCSD & 1667 & 1192 & 72 & $9-97$ & 21 \\
\hline$\checkmark$-Nitrosodimethylamine & L0111248-04-MS & 1667 & 1036 & 62 & $9-97$ & \\
\hline$\checkmark$-Nitrosodimethylamine & L0111248-04-MSD & 1667 & 1053 & 63 & $9-97$ & 1.6 \\
\hline$\checkmark$-Nitrosodi-n-propylamine & $011129-8270 S-L C S$ & 1667 & 1334 & 80 & $41-126$ & \\
\hline$\dot{V}$-Nitrosodi-n-propylamine & 011129-8270S-LCSD & 1667 & 1124 & 67 & $41-126$ & 17.1 \\
\hline J-Nitrosodi-n-propylamine & L0111248-04-MS & 1667 & 958 & 57 & $41-126$ & \\
\hline J-Nitrosodi-n-propylamine & L0111248-04-MSD & 1667 & 1018 & 61 & $41-126$ & 6.1 \\
\hline -Nitrosodiphenylamine & $011129-8270$ S-LCS & 1667 & 1499 & 90 & $50-120$ & \\
\hline J-Nitrosodiphenylamine & $011129-8270 S-L C S D$ & 1667 & 1286 & 77 & $50-120$ & 15.3 \\
\hline
\end{tabular}

$\sqrt{D}$ - Not Detected

his report shall not be reproduced except in full, without the written approval of the laboratory. 
NEL LABORATORIES

\begin{tabular}{ll}
\hline CLIENT: & Bechtel \\
PROJECT ID: & V1358 \\
PROJECT \#: & 30033
\end{tabular}

TEST: $\quad$ Semi-Volatile Organic Compounds by EPA 8270C, Dec. 1996 MATRIX: Solid

\begin{tabular}{|c|c|c|c|c|c|c|c|}
\hline PARAMETER & NEL Sample ID & $\begin{array}{c}\text { Spike } \\
\text { Amount }\end{array}$ & $\frac{\text { Spike }}{\underline{\text { Result }}}$ & $\underline{\text { Percent }}$ & & $\frac{\text { Acceptable }}{\text { Range }}$ & $\underline{\mathbf{R P D}}$ \\
\hline N-Nitrosodiphenylamine & L0111248-04-MS & 1667 & 1272 & 76 & & $50-120$ & \\
\hline N-Nitrosodiphenylamine & L0111248-04-MSD & 1667 & 1300 & 78 & & $50-120$ & 2.2 \\
\hline Pentachlorophenol & $011129-8270 S-L C S$ & 1667 & 862 & 52 & & $17-109$ & \\
\hline Pentachlorophenol & 011129-8270S-LCSD & 1667 & 585 & 35 & R5 & $17-109$ & 38.3 \\
\hline Pentachlorophenol & L0111248-04-MS & 1667 & 1222 & 73 & & $17-109$ & \\
\hline Pentachlorophenol & L0111248-04-MSD & 1667 & 1092 & 66 & & $17-109$ & 11.2 \\
\hline Phenol & $011129-8270$ S-LCS & 1667 & 1308 & 78 & & $52-113$ & \\
\hline Phenol & 011129-8270S-LCSD & 1667 & 1052 & 63 & & $52-113$ & 21.7 \\
\hline Phenol & L0111248-04-MS & 1667 & 951 & 57 & & $52-113$ & \\
\hline Phenol & L0111248-04-MSD & 1667 & 1010 & 61 & & $52-113$ & 6. \\
\hline Phenanthrene & $011129-8270$ S-LCS & 1667 & 1536 & 92 & & $50-120$ & \\
\hline Phenanthrene & $011129-8270$ S-LCSD & 1667 & 1341 & 80 & & $50-120$ & 13.6 \\
\hline Phenanthrene & L0111248-04-MS & 1667 & 1323 & 79 & & $50-120$ & \\
\hline Phenanthrene & L0111248-04-MSD & 1667 & 1399 & 84 & & $50-120$ & 5.6 \\
\hline Pyrene & $011129-8270 S-L C S$ & 1667 & 1534 & 92 & & $50-120$ & \\
\hline Pyrene & $011129-8270 \mathrm{~S}-\mathrm{LCSD}$ & 1667 & 1298 & 78 & & $50-120$ & 16.7 \\
\hline Pyrene & L0111248-04-MS & 1667 & 1249 & 75 & & $50-120$ & \\
\hline Pyrene & L0111248-04-MSD & 1667 & 1374 & 82 & & $50-120$ & 9.5 \\
\hline Pyridine & $011129-8270$ S-LCS & 1667 & 1021 & 61 & & $4-71$ & \\
\hline Pyridine & $011129-8270 S-L C S D$ & 1667 & 818 & 49 & & $4-71$ & 22.1 \\
\hline Pyridine & L0111248-04-MS & 1667 & 738 & 44 & & $4-71$ & \\
\hline Pyridine & L0111248-04-MSD & 1667 & 745 & 45 & & $4-71$ & 0.9 \\
\hline 1,2,4-Trichlorobenzene & $011129-8270$ S-LCS & 1667 & 1387 & 83 & & $38-107$ & \\
\hline 1,2,4-Trichlorobenzene & 011129-8270S-LCSD & 1667 & 1144 & 69 & & $38-107$ & 19.2 \\
\hline 1,2,4-Trichlorobenzene & L0111248-04-MS & 1667 & 1004 & 60 & & $38-107$ & \\
\hline 1,2,4-Trichlorobenzene & L01 $11248-04-M S D$ & 1667 & 1067 & 64 & & $38-107$ & 6.1 \\
\hline 2,4,5-Trichlorophenol & $011129-8270$ S-LCS & 1667 & 1370 & 82 & & $50-120$ & \\
\hline 2,4,5-Trichlorophenol & $011129-8270 S-L C S D$ & 1667 & 1173 & 70 & & $50-120$ & 15.5 \\
\hline 2,4,5-Trichlorophenol & L0111248-04-MS & 1667 & 1303 & 78 & & $50-120$ & \\
\hline 2,4,5-Trichlorophenol & L0111248-04-MSD & 1667 & 1319 & 79 & & $50-120$ & 1.2 \\
\hline 2,4,6-Trichlorophenol & $011129-8270$ S-LCS & 1667 & 1328 & 80 & & $50-120$ & \\
\hline 2,4,6-Trichlorophenol & $011129-8270 S-L C S D$ & 1667 & 951 & 57 & R5 & $50-120$ & 33.1 \\
\hline 2,4,6-Trichlorophenol & L0111248-04-MS & 1667 & 1203 & 72 & & $50-120$ & \\
\hline 2,4,6-Trichlorophenol & L0111248-04-MSD & 1667 & 1268 & 76 & & $50-120$ & 5.3 \\
\hline
\end{tabular}

ND - Not Detected

This report shall not be reproduced except in full, without the written approval of the laboratory. 
NEL LABORATORIES

CLIENT: Bechtel Nevada

PROJECT ID: V1358

PROJECT \#: 30033

TEST:

MATRIX:

Total Extractable Petroleum Hydrocarbons Fuel Finger Print by EPA Method 8015M, July 1992 Solid

\section{PARAMETER}

Diesel Range (C12-C22)

Diesel Range (C12-C22)

Diesel Range (C12-C22)

Diesel Range (C12-C22)

Total

Total

Total

Total
NEL Sample ID

011130TPHS-FP-LCS

011130TPHS-FP-LCSD

L0111248-04-MS

L0111248-04-MSD

011130 TPHS-FP-LCS

011130TPHS-FP-LCSD

L01 11248-04-MS

L0111248-04-MSD

\begin{tabular}{|c|c|c|c|c|}
\hline$\frac{\text { Spike }}{\text { Amount }}$ & $\begin{array}{r}\text { Spike } \\
\text { Result }\end{array}$ & $\frac{\text { Percent }}{\text { Recovery }}$ & $\frac{\text { Acceptable }}{\text { Range }}$ & $\underline{\mathrm{RPD}}$ \\
\hline 166.7 & 120 & 72 & $53-91$ & \\
\hline 166.7 & 95 & 57 & $53-91$ & 23.3 \\
\hline 166.7 & 111 & 67 & $34-114$ & \\
\hline 166.7 & 100 & 60 & $34-114$ & 10.4 \\
\hline 166.7 & 120 & 72 & $53-91$ & \\
\hline 166.7 & 95 & 57 & $53-91$ & 23.3 \\
\hline 166.7 & 111 & 67 & $34-114$ & \\
\hline 166.7 & 100 & 60 & $34-114$ & 10.4 \\
\hline
\end{tabular}

VD - Not Detected

This report shall not be reproduced except in full, without the written approval of the laboratory. 


\begin{tabular}{ll}
\hline CLIENT: & Bechtel Nevada \\
PROJECT ID: & V1358 \\
PROJECT \#: & 30033 \\
TEST: & Volatile Organic Compounds by EPA 8260B, December 1996 \\
MATRIX: & Aqueous
\end{tabular}

\begin{tabular}{|c|c|c|c|c|c|c|}
\hline PARAMETER & NEL Sample ID & $\underset{\text { Ampike }}{\text { Amount }}$ & $\frac{\text { Spike }}{\underline{\text { Result }}}$ & $\frac{\text { Percent }}{\text { Recovery }}$ & $\frac{\text { Acceptable }}{\text { Range }}$ & $\underline{\mathbf{R P D}}$ \\
\hline Acetone & 011202AQ60_2A-LCS & 50 & 52.64 & 105 & $70-130$ & \\
\hline Acetone & L0111210-01-MS & 50 & 42.91 & 86 & $70-130$ & \\
\hline Acetone & L0111210-01-MSD & 50 & 48.26 & 97 & $70-130$ & 11.7 \\
\hline Benzene & 011202AQ60_2A-ICS & 50 & 54.61 & 109 & $70-130$ & \\
\hline Benzene & L0111210-01-MS & 50 & 53.14 & 106 & $70-130$ & \\
\hline Benzene & L0111210-01-MSD & 50 & 50.56 & 101 & $70-130$ & 5. \\
\hline Bromobenzene & 011202AQ60_2A-LCS & 50 & 53.55 & 107 & $70-130$ & \\
\hline Bromobenzene & L0111210-01-MS & 50 & 57.67 & 115 & $70-130$ & \\
\hline Bromobenzene & L0111210-01-MSD & 50 & 53.67 & 107 & $70-130$ & 7.2 \\
\hline Bromochloromethane & 011202AQ60_2A-LCS & 50 & 48.81 & 98 & $70-130$ & \\
\hline Bromochlotomethane & L0111210-01-MS & 50 & 50.94 & 102 & $70-130$ & \\
\hline Bromochloromethane & L0111210-01-MSD & 50 & 48.44 & 97 & $70 \cdot 130$ & 5. \\
\hline Bromodichloromethane & 011202AQ60_2A-LCS & 50 & 48.44 & 97 & $70-130$ & \\
\hline Bromodichloromethane & L0111210-01-MS & 50 & 47.11 & 94 & $70-130$ & \\
\hline Bromodichloromethane & L0111210-01-MSD & 50 & 45.68 & 91 & $70-130$ & 3.1 \\
\hline Bromoform & 011202AQ60_2A-LCS & 50 & 50.78 & 102 & $70-130$ & \\
\hline Bromoform & L0111210-01-MS & 50 & 50.61 & 101 & $70-130$ & \\
\hline Bromoform & L0111210-01-MSD & 50 & 52.28 & 105 & $70-130$ & 3.2 \\
\hline Bromomethane & $011202 \mathrm{AQ} 60 \_2 \mathrm{~A}-\mathrm{LCS}$ & 50 & 48.4 & 97 & $70-130$ & \\
\hline Bromomethane & L0111210-01-MS & 50 & 49.78 & 100 & $70-130$ & \\
\hline Bromomethane & L0111210-01-MSD & 50 & 47.76 & 96 & $70-130$ & 4.1 \\
\hline 2-Butanone & 011202AQ60_2A-LCS & 50 & 54.31 & 109 & $70-130$ & \\
\hline 2-Butanone & L0111210-01-MS & 50 & 43.03 & 86 & $70-130$ & \\
\hline 2-Butanone & L0111210-01-MSD & 50 & 48.56 & 97 & $70-130$ & 12.1 \\
\hline n-Butylbenzene & $011202 \mathrm{AQ60} 2 \mathrm{~A}-\mathrm{LCS}$ & 50 & 53.98 & 108 & $70-130$ & \\
\hline a-Butylbenzene & L0111210-01-MS & 50 & 54.54 & 109 & $70-130$ & \\
\hline 1-Butylbenzene & L0111210-01-MSD & 50 & 53.24 & 106 & $70-130$ & 2.4 \\
\hline sec-Butylbenzene & 011202AQ60_2A-LCS & 50 & 59.52 & 119 & $70-130$ & \\
\hline iec-Butylbenzene & L0111210-01-MS & 50 & 63.1 & 126 & $70-130$ & \\
\hline sec-Butylbenzene & L0111210-01-MSD & 50 & 59.91 & 120 & $70-130$ & 5.2 \\
\hline ert-Butylbenzene & 011202AQ60_2A-ICS & 50 & 56.42 & 113 & $70-130$ & \\
\hline ert-Butylbenzene & L0111210-01-MS & 50 & 60.6 & 121 & $70-130$ & \\
\hline ert-Butylbenzene & L0111210-01-MSD & 50 & 57.66 & 115 & $70-130$ & 5. \\
\hline Zarbon disulfide & $011202 \mathrm{AQ60} 2 \mathrm{~A}-\mathrm{LCS}$ & 50 & 96.17 & 192 & $70-130$ & \\
\hline Zarbon disulfide & L0111210-01-MS & 50 & 94.8 & $190 J$ & $70-130$ & \\
\hline Zarbon disulfide & L0111210-01-MSD & 50 & 87.03 & 174 & $70=130$ & 8.5 \\
\hline Zarbon tetrachloride & 011202AQ60_2A-LCS & 50 & 48.73 & 97 & $70-130$ & \\
\hline Zarbon tetrachloride & L0111210-01-MS & 50 & 53 & 106 & $70-130$ & \\
\hline
\end{tabular}

VD - Not Detected

This report shall not be reproduced except in full, without the written approval of the laboratory. 


\begin{tabular}{ll}
\hline CLIENT: & Bechtel Nevada \\
PROJECT ID: & V1358 \\
PROJECT \#: & 30033 \\
TEST: & Volatile Organic Compounds by EPA 8260B, December 1996 \\
MATRIX: & Aqueous
\end{tabular}

\begin{tabular}{|c|c|c|c|c|c|c|}
\hline PARAMETER & NEL Sample ID & $\underline{\text { Spike }}$ & $\frac{\text { Spike }}{\text { Result }}$ & $\frac{\text { Percent }}{\text { Recovery }}$ & $\frac{\text { Acceptable }}{\underline{\text { Range }}}$ & $\underline{\text { RPD }}$ \\
\hline Carbon tetrachloride & L0111210-01-MSD & 50 & 53.71 & 107 & $70-130$ & 1.3 \\
\hline Chlorobenzene & 011202AQ60_2A-LCS & 50 & 55.25 & 111 & $70-130$ & \\
\hline Chlorobenzene & L0111210-01-MS & 50 & 56.47 & 113 & $70-130$ & \\
\hline Chlorobenzene & L0111210-01-MSD & 50 & 53.46 & 107 & $70-130$ & 5.5 \\
\hline Chloroethane & 011202AQ60_2A-ICS & 50 & 51.66 & 103 & $70-130$ & \\
\hline Chloroethane & L0111210-01-MS & 50 & 46.33 & 93 & $70-130$ & \\
\hline Chloroethane & L0111210-01-MSD & 50 & 43.92 & 88 & $70-130$ & 5.3 \\
\hline Chloroform & 011202AQ60_2A-LCS & 50 & 46.16 & 92 & $70-130$ & \\
\hline Chloroform & L0111210-01-MS & 50 & 43.79 & 88 & $70-130$ & \\
\hline Chloroform & L0111210-01-MSD & 50 & 41.15 & 82 & $70-130$ & 6.2 \\
\hline Chloromethane & 011202AQ60_2A-LCS & 50 & 48.05 & 96 & $70-130$ & \\
\hline Chloromethane & L0111210-01-MS & 50 & 41.31 & 83 & $70-130$ & \\
\hline Chloromethane & L0111210-01-MSD & 50 & 38.4 & 77 & $70-130$ & 7.3 \\
\hline 2-Chlorotoluene & $011202 \mathrm{AQ60} 2 \mathrm{~A}-\mathrm{LCS}$ & 50 & 56.37 & 113 & $70-130$ & \\
\hline 2-Chlorotoluene & L0111210-01-MS & 50 & 56.03 & 112 & $70-130$ & \\
\hline 2-Chlorotoluene & L0111210-01-MSD & 50 & 51.31 & 103 & $70-130$ & 8.8 \\
\hline 4-Chlorotoluene & 011202AQ60_2A-LCS & 50 & 54.09 & 108 & $70-130$ & \\
\hline 4-Chlorotoluene & L0111210-01-MS & 50 & 53.58 & 107 & $70-130$ & \\
\hline 4-Chlorotoluene & L0111210-01-MSD & 50 & 49.42 & 99 & $70-130$ & 8.1 \\
\hline Dibromochloromethane & 011202AQ60_2A-LCS & 50 & 47.95 & 96 & $70-130$ & \\
\hline Dibromochloromethane & L0111210-01-MS & 50 & 48.3 & 97 & $70-130$ & \\
\hline Dibromochloromethane & L0111210-01-MSD & 50 & 48.38 & 97 & $70-130$ & 0.2 \\
\hline 1,2-Dibromo-3-chloropropane (DBCP) & 011202AQ60_2A-LCS & 50 & 48.8 & 98 & $70-130$ & \\
\hline 1,2-Dibromo-3-chloropropane (DBCP) & L0111210-01-MS & 50 & 37.73 & 75 & $70-130$ & \\
\hline 1,2-Dibromo-3-chloropropane (DBCP) & L0111210-01-MSD & 50 & 45.77 & 92 & $70-130$ & 19.3 \\
\hline 1,2-Dibromoethane (EDB) & 011202AQ60_2A-LCS & 50 & 47.98 & 96 & $70-130$ & \\
\hline 1,2-Dibromoethane (EDB) & L0111210-01-MS & 50 & 46.14 & 92 & $70-130$ & \\
\hline 1,2-Dibromoethane (EDB) & L0111210-01-MSD & 50 & 48.09 & 96 & $70-130$ & 4.1 \\
\hline Dibromomethane & 011202AQ60_2A-LCS & 50 & 48.91 & 98 & $70-130$ & \\
\hline Dibromomethane & L0111210-01-MS & 50 & 46.45 & 93 & $70-130$ & \\
\hline Dibromomethane & L0111210-01-MSD & 50 & 46.72 & 93 & $70-130$ & 0.6 \\
\hline 1,2-Dichlorobenzene (o-DCB) & 011202AQ60_2A-LCS & 50 & 51.47 & 103 & $70-130$ & \\
\hline 1,2-Dichlorobenzene (o-DCB) & L0111210-01-MS & 50 & 54.29 & 109 & $70-130$ & \\
\hline 1,2-Dichlorobenzene (o-DCB) & L0111210-01-MSD & 50 & 51.55 & 103 & $70-130$ & 5.2 \\
\hline 1,3-Dichlorobenzene (m-DCB) & 011202AQ60_2A-LCS & 50 & 52.77 & 106 & $70-130$ & \\
\hline 1,3-Dichlorobenzene (m-DCB) & L0111210-01-MS & 50 & 56.89 & 114 & $70-130$ & \\
\hline 1,3-Dichlorobenzene (m-DCB) & L0111210-01-MSD & 50 & 53.58 & 107 & $70-130$ & 6. \\
\hline 1,4-Dichlorobenzene ( $\mathrm{p}-\mathrm{DCB})$ & 011202AQ60_2A-LCS & 50 & 52.87 & 106 & $70-130$ & \\
\hline
\end{tabular}

VD - Not Detected

This report shall not be reproduced except in full, without the written approval of the laboratory. 


\begin{tabular}{ll}
\hline CLIENT: & Bechtel Nevada \\
PROJECT ID: & V1358 \\
PROJECT \#: & 30033 \\
TEST: & Volatile Organic Compounds by EPA 8260B, December 1996 \\
MATRIX: & Aqueous
\end{tabular}

\begin{tabular}{|c|c|c|c|c|c|c|}
\hline PARAMETER & NEL Sample ID & $\underline{\text { Spike }}$ & $\frac{\text { Spike }}{\text { Result }}$ & $\frac{\text { Percent }}{\text { Recovery }}$ & $\frac{\text { Acceptable }}{\text { Range }}$ & $\underline{\mathbf{R P D}}$ \\
\hline 1,4-Dichlorobenzene ( $p-D C B$ ) & L0111210-01-MS & 50 & 57.16 & 114 & $70-130$ & \\
\hline 1,4-Dichlorobenzene ( $\mathrm{p}$-DCB) & L0111210-01-MSD & 50 & 53.66 & 107 & $70-130$ & 6.3 \\
\hline Dichlorodifluoromethane (Freon 12) & 011202AQ60_2A-LCS & 50 & 48.05 & 96 & $70-130$ & \\
\hline Dichlorodifluoromethane (Freon 12) & L0111210-01-MS & 50 & 41.31 & 83 & $70-130$ & \\
\hline Dichlorodifluoromethane (Freon 12) & L0111210-01-MSD & 50 & 38.4 & 77 & $70-130$ & 7.3 \\
\hline 1,1-Dichloroethane (1,1-DCA) & $011202 \mathrm{AQ60} 2 \mathrm{~A}-\mathrm{LCS}$ & 50 & 52.15 & 104 & $70-130$ & \\
\hline 1,1-Dichloroethane (1,1-DCA) & L0111210-01-MS & 50 & 46.4 & 93 & $70-130$ & \\
\hline 1,1-Dichloroethane (1,1-DCA) & L0111210-01-MSD & 50 & 43.86 & 88 & $70-130$ & 5.6 \\
\hline 1,2-Dichloroethane (1,2-DCA) & 011202AQ60_2A-LCS & 50 & 45.88 & 92 & $70-130$ & \\
\hline 1,2-Dichloroethane (1,2-DCA) & L0111210-01-MS & 50 & 38.71 & 77 & $70-130$ & \\
\hline 1,2-Dichloroethane (1,2-DCA) & L0111210-01-MSD & 50 & 38.49 & 77 & $70-130$ & 0.6 \\
\hline 1,1-Dichloroethene (1,1-DCE) & 011202AQ60_2A-LCS & 50 & 52.75 & 106 & $70-130$ & \\
\hline 1,1-Dichioroethene (1,1-DCE) & L0111210-01-MS & 50 & 54.78 & 110 & $70-130$ & \\
\hline 1,1-Dichloroethene (1,1-DCE) & L0111210-01-MSD & 50 & 55.3 & 111 & $70-130$ & 0.9 \\
\hline zis-1,2-Dichloroethene & 011202AQ60_2A-LCS & 50 & 52.31 & 105 & $70-130$ & \\
\hline sis-1,2-Dichloroethene & L0111210-01-MS & 50 & 54.45 & 109 & $70-130$ & \\
\hline sis-1,2-Dichloroethene & L0111210-01-MSD & 50 & 51.23 & 102 & $70-130$ & 6.1 \\
\hline rans-1,2-Dichloroethene & 011202AQ60_2A-LCS & 50 & 53.31 & 107 & $70-130$ & \\
\hline 'rans-1,2-Dichloroethene & L0111210-01-MS & 50 & 57.05 & 114 & $70-130$ & \\
\hline rans-1,2-Dichloroethene & L0111210-01-MSD & 50 & 55.11 & 110 & $70-130$ & 3.5 \\
\hline 1,2-Dichloropropane & 011202AQ60_2A-LCS & 50 & 52.79 & 106 & $70-130$ & \\
\hline 1,2-Dichloropropane & L0111210-01-MS & 50 & 47.16 & 94 & $70-130$ & \\
\hline 1,2-Dichloropropane & L0111210-01-MSD & 50 & 44.6 & 89 & $70-130$ & 5.6 \\
\hline 1,3-Dichloropropane & 011202AQ60_2A-LCS & 50 & 50.58 & 101 & $70-130$ & \\
\hline 1,3-Dichloropropane & L0111210-01-MS & 50 & 45.07 & 90 & $70-130$ & \\
\hline 1,3-Dichloropropane & L0111210-01-MSD & 50 & 43.86 & 88 & $70-130$ & 2.7 \\
\hline 2,2-DichIoropropane & 011202AQ60_2A-LCS & 50 & 53.82 & 108 & $70-130$ & \\
\hline 2,2-Dichloropropane & L0111210-01-MS & 50 & 39.42 & 79 & $70-130$ & \\
\hline 2,2-Dichloropropane & L0111210-01-MSD & 50 & 39.02 & 78 & $70-130$ & 1. \\
\hline 1,1-Dichloropropene & $011202 \mathrm{AQ} 60 \_2 \mathrm{~A}-\mathrm{LCS}$ & 50 & 55.05 & 110 & $70-130$ & \\
\hline 1,1-Dichloropropene & L0111210-01-MS & 50 & 54.77 & 110 & $70-130$ & \\
\hline ,1-Dichloropropene & L0111210-01-MSD & 50 & 53.23 & 106 & $70-130$ & 2.9 \\
\hline :is-1,3-Dichloropropene & 011202AQ60_2A-LCS & 50 & 50.34 & 101 & $70-130$ & \\
\hline :is-1,3-Dichloropropene & L0111210-01-MS & 50 & 46.31 & 93 & $70-130$ & \\
\hline :is-1,3-Dichloropropene & L0111210-01-MSD & 50 & 44.07 & 88 & $70-130$ & 5. \\
\hline rans-1,3-Dichloropropene & 011202AQ60_2A-LCS & 50 & 44.83 & 90 & $70-130$ & \\
\hline rans-1,3-Dichloropropene & L0111210-01-MS & 50 & 39.8 & 80 & $70-130$ & \\
\hline rans-1,3-Dichloropropene & L0111210-01-MSD & 50 & 39.86 & 80 & $70-130$ & 0.2 \\
\hline
\end{tabular}

SD - Not Detected

This report shall not be reproduced except in full, without the written approval of the laboratory. 
NEL Laboratories

CLIENT: Bechtel Nevada

PROJECT ID: V1358

PROJECT \#: 30033

TEST: $\quad$ Volatile Organic Compounds by EPA 8260B, December 1996

MATRLX: Aqueous

\begin{tabular}{|c|c|c|c|c|c|c|c|}
\hline PARAMETER & NEL Sample ID & $\frac{\text { Spike }}{\text { Amount }}$ & Spike & $\frac{\text { Percent }}{\text { Recovery }}$ & & $\frac{\text { Acceptable }}{\underline{\text { Range }}}$ & $\underline{\mathbf{R P D}}$ \\
\hline Ethylbenzene & 011202AQ60_2A-LCS & 50 & 55.09 & 110 & & $70-130$ & \\
\hline Ethylbenzene & L0111210-01-MS & 50 & 58.07 & 116 & & $70-130$ & \\
\hline Ethylbenzene & L0111210-01-MSD & 50 & 54.96 & 110 & & $70-130$ & 5.5 \\
\hline Hexachlorobutadiene & 011202AQ60_2A-LCS & 50 & 46.82 & 94 & & $70-130$ & \\
\hline Hexachlorobutadiene & L0111210-01-MS & 50 & 54.2 & 108 & & $70-130$ & \\
\hline Hexachlorobutadiene & L0111210-01-MSD & 50 & 58.59 & 117 & & $70-130$ & 7.8 \\
\hline 2-Hexanone & 011202AQ60_2A-LCS & 50 & 51.35 & 103 & & $70-130$ & \\
\hline 2-Hexanone & L0111210-01-MS & 50 & 32.91 & 66 & $\mathrm{~J}$ & $70-130$ & \\
\hline 2-Hexanone & L0111210-01-MSD & 50 & 40.34 & 81 & & $70-130$ & 20.3 \\
\hline Iodomethane & $011202 \mathrm{AQ} 60 \_2 \mathrm{~A} \cdot \mathrm{LCS}$ & 50 & 55.51 & 111 & & $70-130$ & \\
\hline lodomethane & L0111210-01-MS & 50 & 58.69 & 117 & & $70-130$ & \\
\hline Iodomethane & L0111210-01-MSD & 50 & 57.53 & 115 & & $70-130$ & 2. \\
\hline isopropylbenzene & 011202AQ60_2A-LCS & 50 & 59.29 & 119 & & $70-130$ & \\
\hline Isopropylbenzene & L0111210-01-MS & 50 & 62.92 & 126 & & $70-130$ & \\
\hline Isopropylbenzene & L0111210-01-MSD & 50 & 56.81 & 114 & & $70-130$ & 10.2 \\
\hline p-Isopropyltoluene & $011202 \mathrm{AQ} 60 \_2 \mathrm{~A}-\mathrm{LCS}$ & 50 & 54.92 & 110 & & $70-130$ & \\
\hline p-Isopropyltoluene & L0111210-01-MS & 50 & 59.57 & 119 & & $70-130$ & \\
\hline p-Isopropyltoluene & L0111210-01-MSD & 50 & 55.84 & 112 & & $70-130$ & 6.5 \\
\hline Methylene chloride (Dichloromethane) & 011202AQ60_2A-LCS & 50 & 49.95 & 100 & & $70-130$ & \\
\hline Methylene chloride (Dichloromethane) & L0111210-01-MS & 50 & 48.21 & 96 & & $70-130$ & \\
\hline Methylene chloride (Dichloromethane) & L0111210-01-MSD & 50 & 46.67 & 93 & & $70 \cdot 130$ & 3.2 \\
\hline 4-Methyl-2-pentanone & 011202AQ60_2A-LCS & 50 & 52.39 & 105 & & $70-130$ & \\
\hline 4-Methyl-2-pentanone & L0111210-01-MS & 50 & 35.74 & 71 & & $70-130$ & \\
\hline 4-Methyl-2-pentanone & L0111210-01-MSD & 50 & 41.1 & 82 & & $70-130$ & 14. \\
\hline MTBE & $011202 \mathrm{AQ} 60 \_2 \mathrm{~A}-\mathrm{LCS}$ & 50 & 49.64 & 99 & & $70-130$ & \\
\hline VTBE & L0111210-01-MS & 50 & 43.42 & 87 & & $70-130$ & \\
\hline VTBE & L0111210-01-MSD & 50 & 43.38 & 87 & & $70-130$ & 0.1 \\
\hline Vaphthalene & $011202 \mathrm{AQ} 60 \_2 \mathrm{~A}-\mathrm{LCS}$ & 50 & 51.77 & 104 & & $70-130$ & \\
\hline Vaphthalene & L0111210-01-MS & 50 & 60.55 & 121 & & $70-130$ & \\
\hline Vaphthalene & L0111210-01-MSD & 50 & 67.24 & 134 & 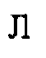 & $70-130$ & 10.5 \\
\hline 1-Propylbenzene & 011202AQ60_2A-LCS & 50 & 58.53 & 117 & & $70-130$ & \\
\hline 1-Propylbenzene & L0111210-01-MS & 50 & 59.69 & 119 & & $70-130$ & \\
\hline 1-Propylbenzene & L0111210-01-MSD & 50 & 55.7 & 111 & & $70-130$ & 6.9 \\
\hline Styrene & 011202AQ60_2A-LCS & 50 & 55.55 & 111 & & $70-130$ & \\
\hline 3tyrene & L0111210-01-MS & 50 & 54.85 & 110 & & $70-130$ & \\
\hline ityrene & L0111210-01-MSD & 50 & 52.4 & 105 & & $70-130$ & 4.6 \\
\hline ,1,1,2-Tetrachloroethane & $011202 \mathrm{AQ} 60$ 2A-LCS & 50 & 50.27 & 101 & & $70-130$ & \\
\hline , 1,1,2-Tetrachloroethane & L0111210-01-MS & 50 & 53.39 & 107 & & $70-130$ & \\
\hline
\end{tabular}

ND - Not Detected

This report shall not be reproduced except in full, without the written approval of the laboratory. 


\begin{tabular}{ll}
\hline CLIENT: & Bechtel Nevada \\
PROJECT ID: & V1358 \\
PROJECT \#: & 30033 \\
TEST: & Volatile Organic Compounds by EPA 8260B, December 1996 \\
MATRIX: & Aqueous
\end{tabular}

\begin{tabular}{|c|c|c|c|c|c|c|c|}
\hline PARAMETER & NEL Sample ID & Spike & $\frac{\text { Spike }}{\text { Result }}$ & $\frac{\text { Percent }}{\text { Recover: }}$ & & $\frac{\text { Acceptable }}{\text { Range }}$ & RPD \\
\hline $1,1,1,2$-Tetrachloroethane & L0111210-01-MSD & 50 & 50.98 & 102 & & $70-130$ & 4.6 \\
\hline 1,1,2,2-Tetrachloroethane & 011202AQ60_2A-LCS & 50 & 53.33 & 107 & & $70-130$ & \\
\hline 1,1,2,2-Tetrachloroethane & L01 11210-01-MS & 50 & 56.66 & 113 & & $70-130$ & \\
\hline 1,1,2,2-Tetrachioroethane & L0111210-01-MSD & 50 & 48.65 & 97 & & $70-130$ & 15.2 \\
\hline Tetrachloroethene (PCE) & 011202AQ60_2A-LCS & 50 & 61.7 & 123 & & $70-130$ & \\
\hline Tetrachloroethene (PCE) & L0111210-01-MS & 50 & 68.53 & 137 & Л & $70-130$ & \\
\hline Tetrachloroethene (PCE) & L0111210-01-MSD & 50 & 66.23 & 132 & $\mathrm{Jl}$ & $70-130$ & 3.4 \\
\hline Toluene & 011202AQ60_2A-LCS & 50 & 53.45 & 107 & & $70-130$ & \\
\hline Toluene & L0111210-01-MS & 50 & 55.14 & 110 & & $70-130$ & \\
\hline Toluene & L0111210-01-MSD & 50 & 51.9 & 104 & & $70-130$ & 6.1 \\
\hline 1,2,3-Trichlorobenzene & $011202 \mathrm{AQ} 60 \_2 \mathrm{~A}-\mathrm{LCS}$ & 50 & 85.55 & 171 & $\mathbf{J}$ & $70-130$ & \\
\hline 1,2,3-Trichlorobenzene & L0111210-01-MS & 50 & 88.96 & 178 & $\mathbf{J}$ & $70-130$ & \\
\hline 1,2,3-Trichlorobenzene & L0I11210-01-MSD & 50 & 111.95 & 224 & $\mathbf{J}$ & $70-130$ & 22.9 \\
\hline 1,2,4-Trichlorobenzene & $011202 \mathrm{AQ} 60 \_2 \mathrm{~A}-\mathrm{LCS}$ & 50 & 49.62 & 99 & & $70-130$ & \\
\hline 1,2,4-Trichlorobenzene & L0111210-01-MS & 50 & 52.07 & 104 & & $70-130$ & \\
\hline 1,2,4-Trichlorobenzene & L0111210-01-MSD & 50 & 58.08 & 116 & & $70-130$ & 10.9 \\
\hline $1,1,1$-Trichloroethane $(1,1,1-\mathrm{TCA})$ & 011202AQ60_2A-LCS & 50 & 45.47 & 91 & & $70-130$ & \\
\hline 1,1,1-Trichloroethane $(1,1,1-\mathrm{TCA})$ & L0111210-01-MS & 50 & 47.34 & 95 & & $70-130$ & \\
\hline 1,1,1-Trichloroethane $(1,1,1-\mathrm{TCA})$ & L0111210-01-MSD & 50 & 46.22 & 92 & & $70-130$ & 2.4 \\
\hline 1,1,2-Trichloroethane $(1,1,2-\mathrm{TCA})$ & 011202AQ60_2A-LCS & 50 & 48.46 & 97 & & $70-130$ & \\
\hline 1,1,2-Trichloroethane $(1,1,2-\mathrm{TCA})$ & L0111210-01-MS & 50 & 46.65 & 93 & & $70-130$ & \\
\hline 1,1,2-Trichloroethane $(1,1,2-\mathrm{TCA})$ & L0111210-01-MSD & 50 & 47.37 & 95 & & $70-130$ & 1.5 \\
\hline Trichloroethene (TCE) & 011202AQ60_2A-LCS & 50 & 53.63 & 107 & & $70-130$ & \\
\hline Trichloroethene (TCE) & L0111210-01-MS & 50 & 56.53 & 113 & & $70-130$ & \\
\hline Trichloroethene (TCE) & L0111210-01-MSD & 50 & 54.72 & 109 & & $70-130$ & 3.3 \\
\hline Trichlorofluoromethane (Freon 11) & 011202AQ60_2A-LCS & 50 & 39.57 & 79 & & $70-130$ & \\
\hline Trichlorofiuoromethane (Freon 11) & L0111210-01-MS & 50 & 38.46 & 77 & & $70-130$ & \\
\hline Trichlorofluoromethane (Freon 11) & L0111210-01-MSD & 50 & 40.85 & 82 & & $70-130$ & 6. \\
\hline 1,2,3-Trichloropropane & 011202AQ60_2A-LCS & 50 & 49.41 & 99 & & $70-130$ & \\
\hline 1,2,3-Trichloropropane & L0111210-01-MS & 50 & 45.92 & 92 & & $70-130$ & \\
\hline 1,2,3-Trichloropropane & L0111210-01-MSD & 50 & 48.13 & 96 & & $70-130$ & 4.7 \\
\hline $1,2,4$-Trimethylbenzene & 011202AQ60_2A-ICS & 50 & 56.41 & 113 & & $70-130$ & \\
\hline 1,2,4-Trimethylbenzene & L0111210-01-MS & 50 & 62.83 & 126 & & $70-130$ & \\
\hline 1,2,4-Trimethylbenzene & L0111210-01-MSD & 50 & 57.42 & 115 & & $70-130$ & 9. \\
\hline 1,3,5-Trimethylbenzene & 011202AQ60_2A-LCS & 50 & 56.41 & 113 & & $70-130$ & \\
\hline 1,3,5-Trimethylbenzene & L0111210-01-MS & 50 & 59.33 & 119 & & $70-130$ & \\
\hline 1,3,5-Trimethylbenzene & L0111210-01-MSD & 50 & 54.68 & 109 & & $70-130$ & 8.2 \\
\hline Jinyl chloride & 011202AQ60_2A-LCS & 50 & 50.56 & 101 & & $70-130$ & \\
\hline
\end{tabular}

VD - Not Detected

This report shall not be reproduced except in full, without the written approval of the laboratory. 
NEL LABORATORIES

\begin{tabular}{ll}
\hline CLIENT: & Bechtel Nevada \\
PROJECT ID: & V1358 \\
PROJECT \#: & 30033 \\
TEST: & Volatile Organic Compounds by EPA 8260B, December 1996 \\
MATRIX: & Aqueous
\end{tabular}

\begin{tabular}{|c|c|c|c|c|c|c|}
\hline PARAMETER & NEL Sample ID & $\frac{\text { Spike }}{\text { Amount }}$ & $\begin{array}{l}\text { Spike } \\
\text { Result }\end{array}$ & $\frac{\text { Percent }}{\text { Recovery }}$ & $\frac{\text { Acceptable }}{\underline{\text { Range }}}$ & $\underline{\mathbf{R P D}}$ \\
\hline Vinyl chloride & L0111210-01-MS & 50 & 45.1 & 90 & $70-130$ & \\
\hline Vinyl chloride & L0111210-01-MSD & 50 & 42.95 & 86 & $70-130$ & 4.9 \\
\hline o-Xylene & $011202 A Q 60 \_2 A-L C S$ & 50 & 54.87 & 110 & $70-130$ & \\
\hline o-Xylene & L0111210-01-MS & 50 & 59.4 & 119 & $70-130$ & \\
\hline o-Xylene & L0111210-01-MSD & 50 & 54.51 & 109 & $70-130$ & 8.6 \\
\hline $\mathrm{m}, \mathrm{p}-\mathrm{Xylene}$ & 011202AQ60_2A-LCS & 100 & 110.69 & 111 & $70-130$ & \\
\hline $\mathrm{m}, \mathrm{p}$-Xylene & L0111210-01-MS & 100 & 119.85 & 120 & $70-130$ & \\
\hline $\mathrm{m}, \mathrm{p}$-Xylene & L0111210-01-MSD & 100 & 113.35 & 113 & $70-130$ & 5.6 \\
\hline
\end{tabular}

ND - Not Detected

This report shall not be reproduced except in full, without the written approval of the laboratory. 


\begin{tabular}{ll}
\hline CLIENT: & Bechtel Nevada \\
PROJECT ID: & V1358 \\
PROJECT \#: & 30033 \\
TEST: & Volatile Organic Compounds by EPA 8260B, December 1996 \\
MATRIX: & Solid
\end{tabular}

\begin{tabular}{|c|c|c|c|c|c|c|c|}
\hline PARAMETER & NEL Sample ID & $\frac{\text { Spike }}{\text { Amount }}$ & $\frac{\text { Spike }}{\text { Result }}$ & $\begin{array}{l}\text { Percent } \\
\text { Recover }\end{array}$ & & $\frac{\text { Acceptable }}{\text { Range }}$ & $\underline{\text { RPD }}$ \\
\hline Acetone & 011202 SD60_1A-LCS & 50 & 74.01 & 148 & $\mathrm{~J}$ & $70-130$ & \\
\hline Acetone & L0111248-04-MS & 50 & 94.41 & 189 & $\mathrm{~J}$ & $70-130$ & \\
\hline Acetone & L01 11248-04-MSD & 50 & 72.03 & 144 & $\mathrm{~J}$ & $70-130$ & 26.9 \\
\hline Benzene & 011202SD60_1A-LCS & 50 & 49.82 & 100 & & $70-130$ & \\
\hline Benzene & L0111248-04-MS & 50 & 49.89 & 100 & & $70-130$ & \\
\hline Benzene & L0111248-04-MSD & 50 & 49.85 & 100 & & $70-130$ & 0.1 \\
\hline Bromobenzene & 011202SD60_1A-LCS & 50 & 46.33 & 93 & & $70-130$ & \\
\hline Bromobenzene & L0111248-04-MS & 50 & 50.47 & 101 & & $70-130$ & \\
\hline Bromobenzene & L0111248-04-MSD & 50 & 49.88 & 100 & & $70-130$ & 1.2 \\
\hline Bromochloromethane & 011202SD60_1A-LCS & 50 & 52.39 & 105 & & $70-130$ & \\
\hline Bromochloromethane & L0111248-04-MS & 50 & 60.36 & 121 & & $70=130$ & \\
\hline Bromochloromethane & L0111248-04-MSD & 50 & 60.44 & 121 & & $70-130$ & 0.1 \\
\hline Bromodichloromethane & 011202SD60_1A-LCS & 50 & 52.23 & 104 & & $70-130$ & \\
\hline Bromodichloromethane & L0111248-04-MS & 50 & 51.19 & 102 & & $70-130$ & \\
\hline Bromodichloromethane & L0111248-04-MSD & 50 & 52.42 & 105 & & $70-130$ & 2.4 \\
\hline Bromoform & 011202SD60_1A-LCS & 50 & 47.09 & 94 & & $70-130$ & \\
\hline Bromoform & L0111248-04-MS & 50 & 55.59 & 111 & & $70-130$ & \\
\hline Bromoform & L0111248-04-MSD & 50 & 49.46 & 99 & & $70-130$ & 11.7 \\
\hline Bromomethane & 011202SD60_1A-LCS & 50 & 53.89 & 108 & & $70-130$ & \\
\hline Bromomethane & L0111248-04-MS & 50 & 57.44 & 115 & & $70-130$ & \\
\hline Bromomethane & L0111248-04-MSD & 50 & 60.69 & 121 & & $70=130$ & 5.5 \\
\hline 2-Butanone & 011202SD60_1A-LCS & 50 & 76.48 & 153 & $\mathrm{~J}$ & $70-130$ & \\
\hline 2-Butanone & L0111248-04-MS & 50 & 102.23 & 204 & $\mathrm{~J}$ & $70-130$ & \\
\hline 2-Butanone & L0111248-04-MSD & 50 & 78.87 & & $\mathbf{J}$ & $70-130$ & 157.7 \\
\hline A-Butylbenzene & 011202SD60_1A-LCS & 50 & 47.62 & 95 & & $70-130$ & \\
\hline 2-Butylbenzene & L0111248-04-MS & 50 & 39.36 & 79 & & $70-130$ & \\
\hline a-Butylbenzene & L0111248-04-MSD & 50 & 38.01 & 76 & & $70-130$ & 3.5 \\
\hline sec-Butylbenzene & 011202SD60_1A-LCS & 50 & 50.49 & 101 & & $70-130$ & \\
\hline sec-Butylbenzene & L0111248-04-MS & 50 & 44.78 & 90 & & $70-130$ & \\
\hline sec-Butylbenzene & L0111248-04-MSD & 50 & 44.13 & 88 & & $70-130$ & 1.5 \\
\hline ert-Butylbenzene & $011202 S D 60 \_1 \mathrm{~A}-\mathrm{LCS}$ & 50 & 47.16 & 94 & & $70-130$ & \\
\hline ert-Butylbenzene & L0111248-04-MS & 50 & 45.83 & 92 & & $70-130$ & \\
\hline ert-Butylbenzene & L0111248-04-MSD & 50 & 44.78 & 90 & & $70-130$ & 2.3 \\
\hline Zarbon disulfide & $011202 \mathrm{SD} 60 \_1 \mathrm{~A}-\mathrm{LCS}$ & 50 & 105.31 & 211 & $\mathrm{~J}$ & $70-130$ & \\
\hline Zarbon disulfide & L0111248-04-MS & 50 & 114.46 & 229 & $\mathrm{~J}$ & $70-130$ & \\
\hline Carbon disulfide & L0111248-04-MSD & 50 & 114.27 & 229 & $\mathrm{~J}$ & $70-130$ & 0.2 \\
\hline Jarbon tetrachloride & 011202SD60_1A-LCS & 50 & 49.52 & 99 & & $70-130$ & \\
\hline Zarbon tetrachloride & L0111248-04-MS & 50 & 48.49 & 97 & & $70-130$ & \\
\hline
\end{tabular}

VD - Not Detected

This report shall not be reproduced except in full, without the written approval of the laboratory. 
NEL LABORATORIES

CLIENT: Bechtel Nevada

PROJECT ID: V1358

PROJECT \#: 30033

TEST: $\quad$ Volatile Organic Compounds by EPA 8260B, December 1996

MATRIX: Solid

\begin{tabular}{|c|c|c|c|c|c|c|c|}
\hline PARAMETER & NEL Sample ID & $\frac{\text { Spike }}{\text { Amount }}$ & $\begin{array}{r}\text { Spike } \\
\underline{\text { Result }}\end{array}$ & $\frac{\text { Percent }}{\text { Recovery }}$ & & $\frac{\text { Acceptable }}{\underline{\text { Range }}}$ & $\underline{\text { RPD }}$ \\
\hline Carbon tetrachloride & $\overline{\text { L0111248-04-MSD }}$ & 50 & 48.52 & 97 & & $70-130$ & 0.1 \\
\hline Chlorobenzene & 011202SD60_1A-LCS & 50 & 49.6 & 99 & & $70-130$ & \\
\hline Chlorobenzene & L0111248-04-MS & 50 & 51.34 & 103 & & $70-130$ & \\
\hline Chlorobenzene & L0111248-04-MSD & 50 & 50.21 & 100 & & $70-130$ & 2.2 \\
\hline Chloroethane & 011202SD60_1A-LCS & 50 & 50.52 & 101 & & $70-130$ & \\
\hline Chloroethane & L0111248-04-MS & 50 & 58.51 & 117 & & $70-130$ & \\
\hline Chloroethane & L0111248-04-MSD & 50 & 61.36 & 123 & & $70-130$ & 4.8 \\
\hline Chloroform & 011202SD60_1A-LCS & 50 & 54.67 & 109 & & $70-130$ & \\
\hline Chloroform & L0111248-04-MS & 50 & 60.84 & 122 & & $70-130$ & \\
\hline Chloroform & L0111248-04-MSD & 50 & 62.15 & 124 & & $70-130$ & 2.1 \\
\hline Chloromethane & 011202 SD60_1A-LCS & 50 & 63.57 & 127 & & $70-130$ & \\
\hline Chloromethane & L0I11248-04-MS & 50 & 64.37 & 129 & & $70-130$ & \\
\hline Chloromethane & L0111248-04-MSD & 50 & 66.17 & 132 & $\mathrm{Jl}$ & $70-130$ & 2.8 \\
\hline 2-Chlorotoluene & 011202SD60_1A-LCS & 50 & 46.39 & 93 & & $70-130$ & \\
\hline 2-Chlorotoluene & L0111248-04-MS & 50 & 47.2 & 94 & & $70-130$ & \\
\hline 2-Chlorotoluene & L0111248-04-MSD & 50 & 48.21 & 96 & & $70-130$ & 2.1 \\
\hline 4-Chlorotoluene & 011202SD60_1A-LCS & 50 & 45.9 & 92 & & $70-130$ & \\
\hline 4-Chlorotoluene & L0111248-04-MS & 50 & 47.77 & 96 & & $70-130$ & \\
\hline 4-Chlorotoluene & L0111248-04-MSD & 50 & 46.09 & 92 & & $70-130$ & 3.6 \\
\hline Dibromochloromethane & 011202SD60_1A-LCS & 50 & 51.58 & 103 & & $70-130$ & \\
\hline Dibromochloromethane & L0111248-04-MS & 50 & 52.11 & 104 & & $70-130$ & \\
\hline Dibromochloromethane & L0111248-04-MSD & 50 & 50.75 & 101 & & $70-130$ & 2.6 \\
\hline 1,2-Dibromo-3-chloropropane (DBCP) & 011202SD60_1A-LCS & 50 & 60.58 & 121 & & $70-130$ & \\
\hline 1,2-Dibromo-3-chtoropropane (DBCP) & L011 I248-04-MS & 50 & 82.73 & 165 & $\pi$ & $70-130$ & \\
\hline 1,2-Dibromo-3-chloropropane (DBCP) & L0111248-04-MSD & 50 & 69.81 & 140 & $\pi$ & $70-130$ & 16.9 \\
\hline 1,2-Dibromoethane (EDB) & 011202SD60_1A-LCS & 50 & 49.69 & 99 & & $70-130$ & \\
\hline 1,2-Dibromoethane (EDB) & L0111248-04-MS & 50 & 52.23 & 104 & & $70-130$ & \\
\hline 1,2-Dibromoethane (EDB) & L0111248-04-MSD & 50 & 48.63 & 97 & & $70-130$ & 7.1 \\
\hline Dibromomethane & 011202SD60_1A-LCS & 50 & 48.9 & 98 & & $70-130$ & \\
\hline Dibromomethane & L0111248-04-MS & 50 & 51.2 & 102 & & $70-130$ & \\
\hline Jibromomethane & L0111248-04-MSD & 50 & 50.09 & 100 & & $70-130$ & 2.2 \\
\hline 1,2-Dichlorobenzene (o-DCB) & 011202SD60_1A-LCS & 50 & 46.1 & 92 & & $70-130$ & \\
\hline 1,2-Dichlorobenzene (o-DCB) & L0111248-04-MS & 50 & 45.82 & 92 & & $70-130$ & \\
\hline !,2-Dichlorobenzene (o-DCB) & L0111248-04-MSD & 50 & 45.1 & 90 & & $70-130$ & 1.6 \\
\hline I,3-Dichlorobenzene (m-DCB) & 011202SD60_1A-LCS & 50 & 46.71 & 93 & & $70-130$ & \\
\hline 1,3-Dichlorobenzene (m-DCB) & L0111248-04-MS & 50 & 46.18 & 92 & & $70-130$ & \\
\hline 1,3-Dichlorobenzene (m-DCB) & L01 11248-04-MSD & 50 & 46.18 & 92 & & $70-130$ & 0. \\
\hline 1,4-Dichlorobenzene (p-DCB) & $011202 S D 60 \_1 A-L C S$ & 50 & 47.27 & 95 & & $70-130$ & \\
\hline
\end{tabular}

VD - Not Detected

This report shall not be reproduced except in full, without the written approval of the laboratory. 


\begin{tabular}{ll}
\hline CLIENT: & Bechtel Nevada \\
PROJECT ID: & V1358 \\
PROJECT \#: & 30033 \\
TEST: & Volatile Organic Compounds by EPA 8260B, December 1996 \\
MATRIX: & Solid
\end{tabular}

\section{PARAMETER}

1,4-Dichlorobenzene (p-DCB)

1,4-Dichlorobenzene ( $\mathrm{p}-\mathrm{DCB}$ )

Dichlorodifluoromethane (Freon 12)

Dichlorodifluoromethane (Freon 12)

Dichlorodifluoromethane (Freon 12)

1,1-Dichloroethane (1,1-DCA)

1,1-Dichloroethane (1,1-DCA)

1,1-Dichloroethane (1,1-DCA)

1,2-Dichloroethane (1,2-DCA)

1,2-Dichloroethane (1,2-DCA)

1,2-Dichloroethane (1,2-DCA)

1,1-Dichloroethene (1,1-DCE)

1,1-Dichloroethene (1,1-DCE)

1,1-Dichloroethene (1,1-DCE)

cis-1,2-Dichloroethene

cis-1,2-Dichloroethene

cis-1,2-Dichloroethene

trans-1,2-Dichloroethene

trans-1,2-Dichloroethene

trans-1,2-Dichloroethene

1,2-Dichloropropane

1,2-Dichloropropane

1,2-Dichloropropane

1,3-Dichloropropane

1,3-Dichloropropane

1,3-Dichloropropane

2,2-Dichloropropane

2,2-Dichloropropane

2,2-Dichloropropane

1,1-Dichloropropene

1,1-Dichloropropene

1,1-Dichloropropene

cis-1,3-Dichloropropene

cis-1,3-Dichloropropene

cis-1,3-Dichloropropene

trans-1,3-Dichloropropene

trans-1,3-Dichloropropene

trans-1,3-Dichloropropene
NEL Sample ID

L0111248-04-MS

L0111248-04-MSD

011202SD60_1A-LCS

L0111248-04-MS

L0111248-04-MSD

011202SD60_1A-LCS

L0111248-04-MS

L0111248-04-MSD

011202SD60_1A-LCS

L0111248-04-MS

L011 1248-04-MSD

011202SD60_1A-LCS

L0111248-04-MS

L0111248-04-MSD

011202SD60_1A-LCS

L0111248-04-MS

L0111248-04-MSD

011202SD60_1A-LCS

L0111248-04-MS

L0111248-04-MSD

011202 SD60_1A-LCS

L0111248-04-MS

L0111248-04-MSD

$011202 S D 60 \_1 \mathrm{~A}-\mathrm{LCS}$

L0111248-04-MS

L0111248-04-MSD

$011202 S D 60 \_I A-L C S$

L0111248-04-MS

L0111248-04-MSD .

011202SD60_1A-LCS

L0I 11248-04-MS

L0111248-04-MSD

011202 SD60_1A-LCS

L0111248-04-MS

L0111248-04-MSD

011202SD60_1A-LCS

L0111248-04-MS

L0111248-04-MSD

\begin{tabular}{|c|c|c|}
\hline$\frac{\text { Spike }}{\text { Amount }}$ & $\frac{\text { Spike }}{\underline{\text { Result }}}$ & $\underline{\text { Percent }}$ \\
\hline 50 & 47.82 & 96 \\
\hline 50 & 46.8 & 94 \\
\hline 50 & 60.74 & 121 \\
\hline 50 & 62.99 & 126 \\
\hline 50 & 64.8 & 130 \\
\hline 50 & 54.72 & 109 \\
\hline 50 & 62.14 & 124 \\
\hline 50 & 64.39 & 129 \\
\hline 50 & 47.75 & 96 \\
\hline 50 & 49.65 & 99 \\
\hline 50 & 50.35 & 101 \\
\hline 50 & 55.72 & 111 \\
\hline 50 & 63.17 & 126 \\
\hline 50 & 62.51 & 125 \\
\hline 50 & 56.71 & 113 \\
\hline 50 & 64.49 & 129 \\
\hline 50 & 64.19 & 128 \\
\hline 50 & 55.1 & 110 \\
\hline 50 & 63.5 & 127 \\
\hline 50 & 63.49 & 127 \\
\hline 50 & 51.98 & 104 \\
\hline 50 & 52.25 & 105 \\
\hline 50 & 51.44 & 103 \\
\hline 50 & 46.81 & 94 \\
\hline 50 & 51.99 & 104 \\
\hline 50 & 50.75 & 101 \\
\hline 50 & 46.08 & 92 \\
\hline 50 & 57.19 & 114 \\
\hline 50 & 58.27 & 117 \\
\hline 50 & 52.29 & 105 \\
\hline 50 & 52.3 & 105 \\
\hline 50 & 51.59 & 103 \\
\hline 50 & 51.03 & 102 \\
\hline 50 & 47.42 & 95 \\
\hline 50 & 46.79 & 94 \\
\hline 50 & 46.85 & 94 \\
\hline 50 & 45.99 & 92 \\
\hline 50 & 44.87 & 90 \\
\hline
\end{tabular}

Acceptable

Range $\underline{\text { RPD }}$

$70-130$

$70-130$

2.2

$70-130$

$70-130$

$70-130$

$70-130$

$70-130$

$70-130$

3.6

$70-130$

$70-130$

$70-130$

1.4

$70-130$

$70-130$

$70-130$

1.1

$70-130$

$70-130$

$70-130$

0.5

$70-130$

$70-130$

$70-130$

0.

$70-130$

$70=130$

$70-130$

1.6

$70-130$

$70-130$

$70-130$

2.4

$70-130$

$70-130$

$70-130$

$70-130$

$70-130$

$70-130$

1.4

$70-130$

$70-130$

$70-130$

$70-130$

$70-130$

$70-130$

2.5

ND - Not Detected

This report shall not be reproduced except in full, without the written approval of the laboratory. 


\begin{tabular}{ll}
\hline CLIENT: & Bechtel Nevada \\
PROJECT ID: & V1358 \\
PROJECT \#: & 30033 \\
TEST: & Volatile Organic Compounds by EPA 8260B, December 1996 \\
MATRIX: & Solid
\end{tabular}

MATRIX: $\quad$ Solid

\begin{tabular}{|c|c|c|c|c|c|c|c|}
\hline PARAMETER & NEL Sample ID & $\frac{\text { Spike }}{\text { Amount }}$ & $\begin{array}{l}\text { Spike } \\
\text { Result }\end{array}$ & $\underline{\text { Percent }}$ & & $\frac{\text { Acceptable }}{\text { Range }}$ & $\underline{\mathrm{RPD}}$ \\
\hline Ethylbenzene & 011202SD60_1A-LCS & 50 & 48.76 & 98 & & $70-130$ & \\
\hline Ethylbenzene & L0111248-04-MS & 50 & 48.96 & 98 & & $70-130$ & \\
\hline Ethylbenzene & L0111248-04-MSD & 50 & 47.98 & 96 & & $70-130$ & 0. \\
\hline Hexachlorobutadiene & 011202SD60_1A-LCS & 50 & 45.97 & 92 & & $70-130$ & \\
\hline Hexachlorobutadiene & L0111248-04-MS & 50 & 24.07 & 48 & $\mathrm{Jl}$ & $70-130$ & \\
\hline Hexachlorobutadiene & L0111248-04-MSD & 50 & 27.29 & 55 & $\pi$ & $70-130$ & 12.5 \\
\hline 2-Hexanone & 011202 SD60_1A-LCS & 50 & 81.54 & .163 & $\mathrm{~J}$ & $70-130$ & \\
\hline 2-Hexanone & L0111248-04-MS & 50 & 69.12 & 138 & J & $70-130$ & \\
\hline 2-Hexanone & L0111248-04-MSD & 50 & 51.79 & 104 & & $70-130$ & 28.7 \\
\hline Iodomethane & 011202SD60_1A-LCS & 50 & 56.76 & 114 & & $70-130$ & \\
\hline Iodomethane & L0111248-04-MS & 50 & 59.38 & 119 & & $70-130$ & \\
\hline Iodomethane & L0111248-04-MSD & 50 & 56.54 & 113 & & $70-130$ & 4.9 \\
\hline Isopropylbenzene & 011202SD60_1A-LCS & 50 & 48.94 & 98 & & $70-130$ & \\
\hline Isopropylbenzene & L0111248-04-MS & 50 & 50.85 & 102 & & $70-130$ & \\
\hline Isopropylbenzene & L0111248-04-MSD & 50 & 49.67 & 99 & & $70-130$ & 2.3 \\
\hline p-Isopropyltoluene & $011202 S D 60 \_1 \mathrm{~A}-\mathrm{LCS}$ & 50 & 47.62 & 95 & & $70-130$ & \\
\hline p-Isopropyltoluene & L0111248-04-MS & 50 & 42.59 & 85 & & $70-130$ & \\
\hline p-Isopropyltoluene & L0111248-04-MSD & 50 & 42.3 & 85 & & $70=130$ & 0.7 \\
\hline Methylene chloride (Dichloromethane) & 011202SD60_1A-LCS & 50 & 52.87 & 106 & & $70-130$ & \\
\hline Methylene chloride (Dichloromethane) & L0111248-04-MS & 50 & 60.51 & 121 & & $70-130$ & \\
\hline Methylene chloride (Dichloromethane) & L0111248-04-MSD & 50 & 61.66 & 123 & & $70-130$ & 1.9 \\
\hline 4-Methyl-2-pentanone & $011202 S D 60 \_1 \mathrm{~A}-\mathrm{LCS}$ & 50 & 73.35 & 147 & $\mathrm{~J}$ & $70-130$ & \\
\hline 4-Methyl-2-pentanone & L0111248-04-MS & 50 & 77.06 & 154 & $J$ & $70-130$ & \\
\hline 4-Methyl-2-pentanone & L0111248-04-MSD & 50 & 66.63 & 133 & $\mathrm{~J}$ & $70-130$ & 14.5 \\
\hline MTBE & 011202SD60_1A-LCS & 50 & 54.86 & 110 & & $70-130$ & \\
\hline MTBE & L0111248-04-MS & 50 & 66.5 & 133 & $\mathrm{n}$ & $70-130$ & \\
\hline MTBE & L0111248-04-MSD & 50 & 64.19 & 128 & & $70-130$ & 3.5 \\
\hline Naphthalene & $011202 S D 60 \_1 \mathrm{~A}-\mathrm{LCS}$ & 50 & 61.37 & 123 & & $70-130$ & \\
\hline Naphthalene & L0111248-04-MS & 50 & 62.42 & 125 & & $70-130$ & \\
\hline Naphthalene & L0111248-04-MSD & 50 & 61.81 & 124 & & $70-130$ & 1. \\
\hline 7-Propylbenzene & $011202 S D 60 \_1 \mathrm{~A}-\mathrm{LCS}$ & 50 & 47.43 & 95 & & $70-130$ & \\
\hline '-Propylbenzene & L0111248-04-MS & 50 & 46.69 & 93 & & $70-130$ & \\
\hline 1-Propylbenzene & L0111248-04-MSD & 50 & 45.92 & 92 & & $70-130$ & 1.7 \\
\hline Styrene & 011202 SD60_1A-LCS & 50 & 50.15 & 100 & & $70-130$ & \\
\hline Styrene & L0111248-04-MS & 50 & 50.87 & 102 & & $70-130$ & \\
\hline Styrene & L0111248-04-MSD & 50 & 49.31 & 99 & & $70-130$ & 3.1 \\
\hline 1,1,1,2-Tetrachloroethane & 011202 SD60_1A-LCS & 50 & 47.34 & 95 & & $70-130$ & \\
\hline 1,1,1,2-Tetrachloroethane & L0111248-04-MS & 50 & 49.98 & 100 & & $70-130$ & \\
\hline
\end{tabular}

VD - Not Detected

This report shall not be reproduced except in full, without the written approval of the laboratory. 


\begin{tabular}{ll}
\hline CLIENT: & Bechtel Nevada \\
PROJECT ID: & V1358 \\
PROJECT \#: & 30033 \\
TEST: & Volatile Organic Compounds by EPA 8260B, December 1996 \\
MATRIX: & Solid
\end{tabular}

\begin{tabular}{|c|c|c|c|c|c|c|c|}
\hline PARAMETER & NEL Sample ID & $\frac{\text { Spike }}{\text { Amount }}$ & $\frac{\text { Spike }}{\text { Result }}$ & $\underline{\text { Percent }}$ & & $\frac{\text { Acceptable }}{\underline{\text { Range }}}$ & $\underline{\text { RPD }}$ \\
\hline 1,1,1,2-Tetrachloroethane & L0111248-04-MSD & 50 & 49.6 & 99 & & $70-130$ & 0.8 \\
\hline 1,1,2,2-Tetrachloroethane & 011202SD60_1A-LCS & 50 & 50.6 & 101 & & $70-130$ & \\
\hline 1,1,2,2-Tetrachloroethane & L0111248-04-MS & 50 & 62.54 & 125 & & $70-130$ & \\
\hline 1,1,2,2-Tetrachloroethane & L0111248-04-MSD & 50 & 52.84 & 106 & & $70-130$ & 16.8 \\
\hline Tetrachloroethene (PCE) & 011202SD60_1A-LCS & 50 & 47.83 & 96 & & $70-130$ & \\
\hline Tetrachloroethene (PCE) & L0111248-04-MS & 50 & 47.73 & 95 & & $70-130$ & \\
\hline Tetrachloroethene (PCE) & L0111248-04-MSD & 50 & 45.97 & 92 & & $70-130$ & 3.8 \\
\hline Toluene & 011202SD60_1A-LCS & 50 & 51.16 & 102 & & $70-130$ & \\
\hline Toluene & L0111248-04-MS & 50 & 50.72 & 101 & & $70-130$ & \\
\hline Toluene & L0111248-04-MSD & 50 & 49.2 & 98 & & $70-130$ & 3. \\
\hline 1,2,3-Trichlorobenzene & 011202SD60_1A-LCS & 50 & 59.03 & 118 & & $70-130$ & \\
\hline 1,2,3-Trichlorobenzene & L0111248-04-MS & 50 & 39.23 & 78 & & $70-130$ & \\
\hline 1,2,3-Trichlorobenzene & L0111248-04-MSD & 50 & 46 & 92 & & $70-130$ & 15.9 \\
\hline 1,2,4-Trichlorobenzene & $011202 S D 60 \_1 \mathrm{~A}-\mathrm{LCS}$ & 50 & 45.16 & 90 & & $70-130$ & \\
\hline 1,2,4-Trichlorobenzene & L0111248-04-MS & 50 & 33.01 & 66 & $\pi$ & $70-130$ & \\
\hline 1,2,4-Trichlorobenzene & L0111248-04-MSD & 50 & 36.09 & 72 & & $70-130$ & 8.9 \\
\hline 1,1,1-Trichloroethane $(1,1,1-\mathrm{TCA})$ & 011202SD60_1A-LCS & 50 & 51.37 & 103 & & $70-130$ & \\
\hline 1,1,1-Trichloroethane $(1,1,1-\mathrm{TCA})$ & L0111248-04-MS & 50 & 59.31 & 119 & & $70-130$ & \\
\hline 1,1,1-Trichloroethane (1,1,1-TCA) & L0111248-04-MSD & 50 & 58.72 & 117 & & $70-130$ & 1. \\
\hline 1,1,2-Trichloroethane $(1,1,2-\mathrm{TCA})$ & $011202 S D 60 \_1 \mathrm{~A}-\mathrm{LCS}$ & 50 & 51.36 & 103 & & $70-130$ & \\
\hline 1,1,2-Trichloroethane $(1,1,2$-TCA $)$ & L0111248-04-MS & 50 & 53.92 & 108 & & $70-130$ & \\
\hline 1,1,2-Trichloroethane $(1,1,2-\mathrm{TCA})$ & L0111248-04-MSD & 50 & 50.97 & 102 & & $70-130$ & 5.6 \\
\hline Trichioroethene (TCE) & 011202SD60_1A-LCS & 50 & 51.02 & 102 & & $70-130$ & \\
\hline Trichloroethene (TCE) & L0111248-04-MS & 50 & 52.6 & 105 & & $70-130$ & \\
\hline Trichloroethene (TCE) & L0111248-04-MSD & 50 & 53.07 & 106 & & $70-130$ & 0.9 \\
\hline Trichlorofluoromethane (Freon 11) & $011202 S D 60 \_1 A-L C S$ & 50 & 66.72 & 133 & $J$ & $70-130$ & \\
\hline Trichlorofluoromethane (Freon 11) & L0111248-04-MS & 50 & 70.45 & 141 & $J$ & $70-130$ & \\
\hline Trichlorofluoromethane (Freon 11) & L0111248-04-MSD & 50 & 71.98 & 144 & $\mathrm{~J}$ & $70-130$ & 2.1 \\
\hline 1,2,3-Trichloropropane & 011202SD60_1A-LCS & 50 & 52.87 & 106 & & $70-130$ & \\
\hline 1,2,3-Trichloropropane & L0111248-04-MS & 50 & 73.81 & 148 & $\pi$ & $70-130$ & \\
\hline 1,2,3-Trichloropropane & L0111248-04-MSD & 50 & 61.92 & 124 & & $70-130$ & 17.5 \\
\hline $1,2,4$-Trimethylbenzene & $011202 S D 60 \_1 \mathrm{~A}-\mathrm{LCS}$ & 50 & 47.12 & 94 & & $70-130$ & \\
\hline 1,2,4-Trimethylbenzene & L0111248-04-MS & 50 & 49.03 & 98 & & $70-130$ & \\
\hline 1,2,4-Trimethylbenzene & L0111248-04-MSD & 50 & 48.08 & 96 & & $70-130$ & 2. \\
\hline 1,3,5-Trimethylbenzene & $011202 S D 60 \_1 \mathrm{~A}-\mathrm{LCS}$ & 50 & 46.92 & 94 & & $70-130$ & \\
\hline 1,3,5-Trimethylbenzene & L0111248-04-MS & 50 & 47.44 & 95 & & $70-130$ & \\
\hline 1,3,5-Trimethylbenzene & L0111248-04-MSD & 50 & 46.91 & 94 & & $70-130$ & 1.1 \\
\hline Vinyl chloride & $011202 S D 60 \_1 \mathrm{~A}-\mathrm{LCS}$ & 50 & 56.07 & 112 & & $70-130$ & \\
\hline
\end{tabular}

VD - Not Detected

This report shall not be reproduced except in full, without the written approval of the laboratory. 
NEL LABORATORIES

CLIENT: Bechtel Nevada

PROJECT ID: V1358

PROJECT \#: 30033

IEST: $\quad$ Volatile Organic Compounds by EPA 8260B, December 1996

MATRIX: Solid

\section{PARAMETER}

Vinyl chloride

Vinyl chioride

o-Xylene

o-Xylene

o-Xylene

m,p-Xylene

$\mathrm{m}, \mathrm{p}-\mathrm{X}$ ylene

m,p-Xylene
NEL Sample ID

L01 1 1248-04-MS

L0111248-04-MSD

011202SD60_1A-LCS

L0111248-04-MS

L0111248-04-MSD

$011202 S D 60 \_1 \mathrm{~A}-\mathrm{LCS}$

L0111248-04-MS

L0111248-04-MSD

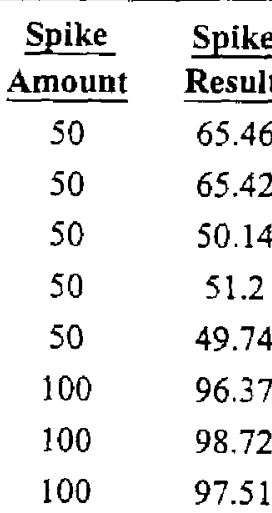

\begin{tabular}{|c|c|c|}
\hline Percent & Acceptable & \\
\hline Recovery & Range & $\underline{\text { RPD }}$ \\
\hline $131 \mathrm{~J}$ & $70-130$ & \\
\hline $131 \mathrm{~J}$ & $70-130$ & 0.1 \\
\hline 100 & $70-130$ & \\
\hline 102 & $70-130$ & \\
\hline 99 & $70-130$ & 2.9 \\
\hline 96 & $70-130$ & \\
\hline 99 & $70-130$ & \\
\hline 98 & $70-130$ & 1.2 \\
\hline
\end{tabular}

- 


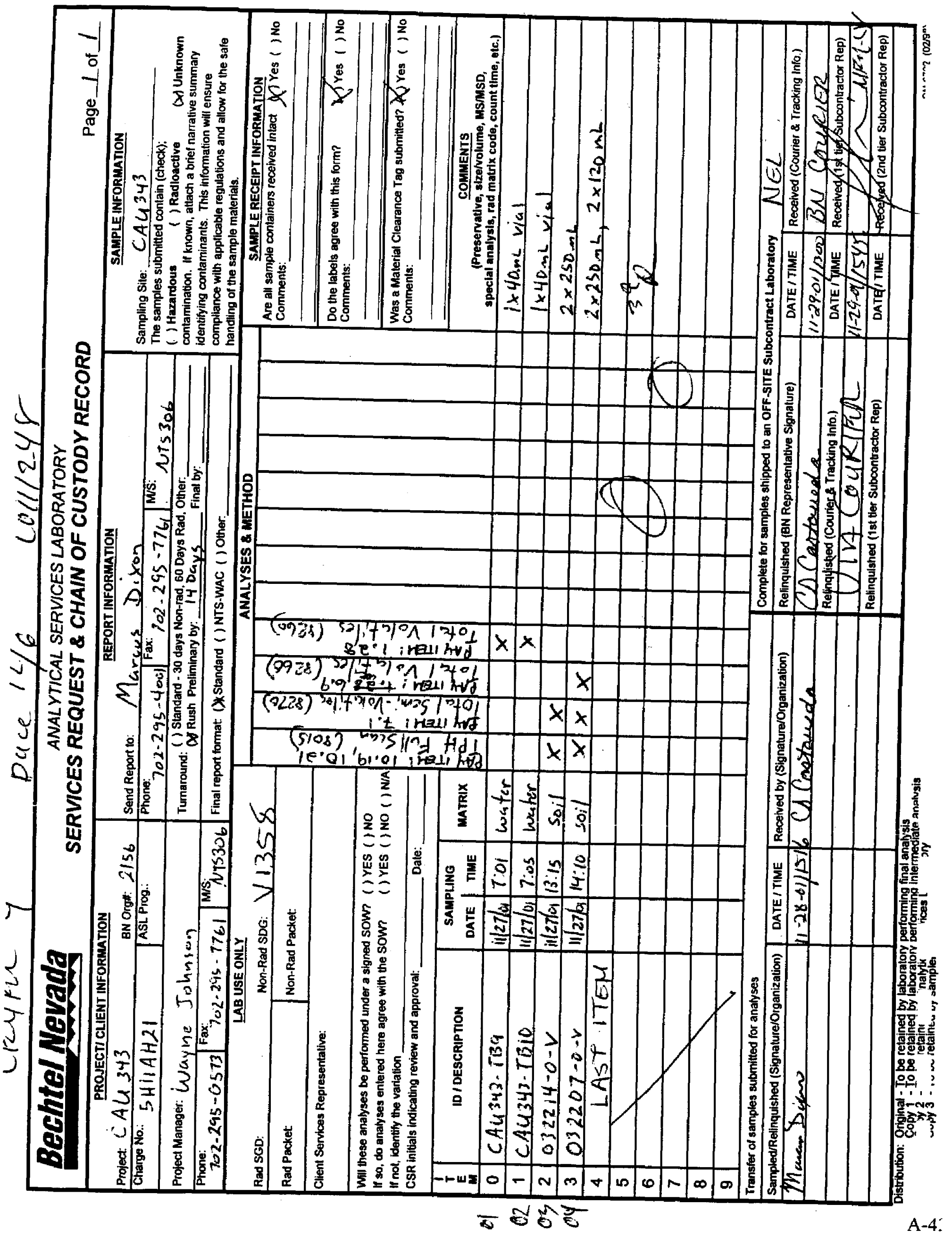




\section{APPENDIX B SECTORED HOUSEKEEPING SITE CLOSURE VERIFICATION FORMS}


THIS PAGE INTENTIONALLY LEFT BLANK 


\section{APPENDIX B TABLE OF CONTENTS}

Sectored Housekeeping Closure Form for CAS 01-24-03 (50-caliber brass shell casings) . . B-1 Sectored Housekeeping Closure Form for CAS 01-24-03 (pieces of lead battery) . . . . . . . B-2 Sectored Housekeeping Closure Form for CAS 03-14-05 . . . . . . . . . . . . B-3 Sectored Housekeeping Closure Form for CAS 03-22-06 . . . . . . . . . . . . . B-4 Sectored Housekeeping Closure Form for CAS 03-22-07 (buckets containing tar) . . . . . . B-5 Sectored Housekeeping Closure Form for CAS 03-22-07 (scattered debris) . . . . . . . . B-6 Sectored Housekeeping Closure Form for CAS 03-22-10 . . . . . . . . . . . B-7

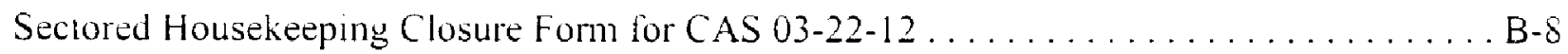
Sectored Housekeeping Closure Form for CAS $03-22-14 \ldots \ldots \ldots \ldots \ldots \ldots \ldots \ldots$

Sectored Housekeeping Closure Form for CAS $03-22-24 \ldots \ldots \ldots \ldots \ldots \ldots \ldots \ldots$. . . . . . . . . . .

Sectored Housekeeping Closure Form for CAS 03-22-25 . . . . . . . . . . . . B-11

Sectored Housekeeping Closure Form for CAS $03-22-26 \ldots \ldots \ldots \ldots \ldots \ldots \ldots \ldots$. . . . . . . . .

Sectored Housekeeping Closure Form for CAS 03-22-30 . . . . . . . . . . . . . B-13

Sectored Housekeeping Closure Form for CAS $03-22-34 \ldots \ldots \ldots \ldots \ldots \ldots \ldots . . . . . . .14$

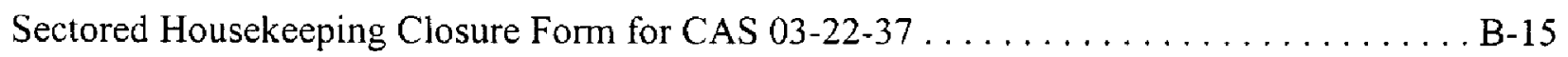

Sectored Housekeeping Closure Form for CAS 03-24-08 . . . . . . . . . . . . . . B-16

Sectored Housekeeping Closure Form for CAS 03-99-10 . . . . . . . . . . . . . . B-17 
THIS PAGE INTENTIONALLY LEFT BLANK 


\section{Sectored Housekeeping Site Closure Verification Farm}

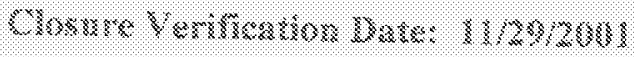

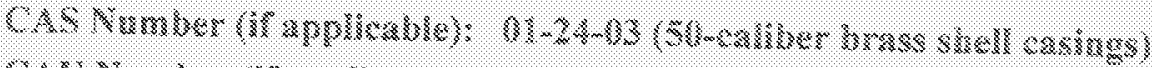

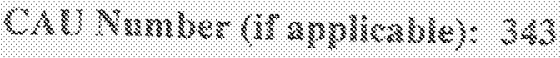

Sexurr Dexignanion: NTS

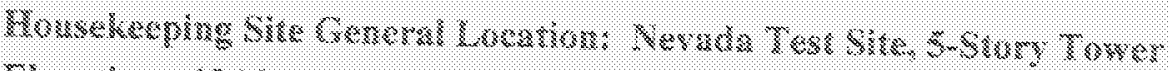

Theration: 1246 meters (rat)

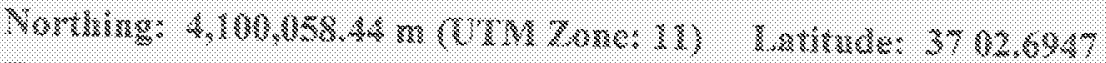

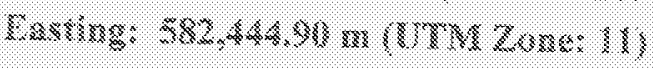

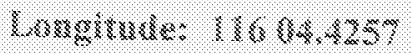

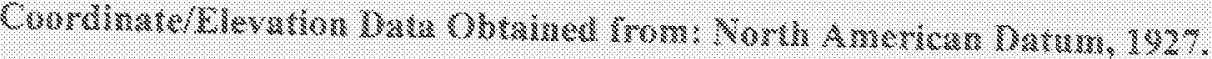

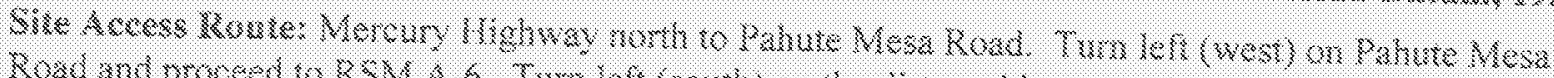

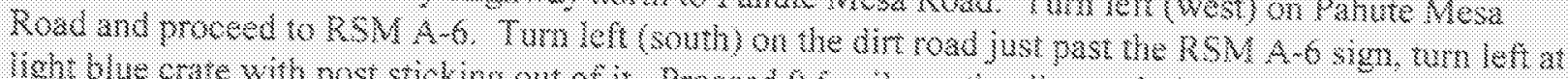

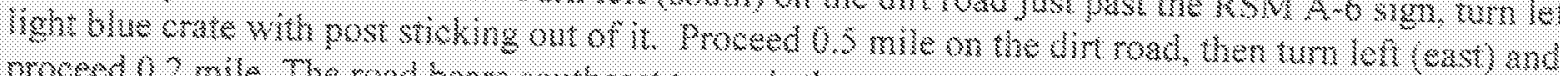

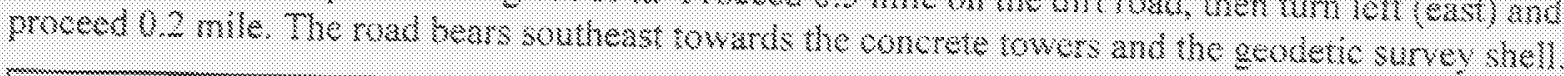

\begin{tabular}{|c|c|}
\hline 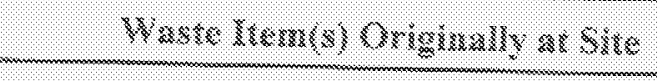 & 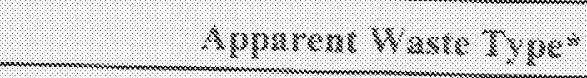 \\
\hline 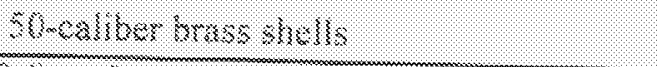 & (oruinzary \\
\hline
\end{tabular}

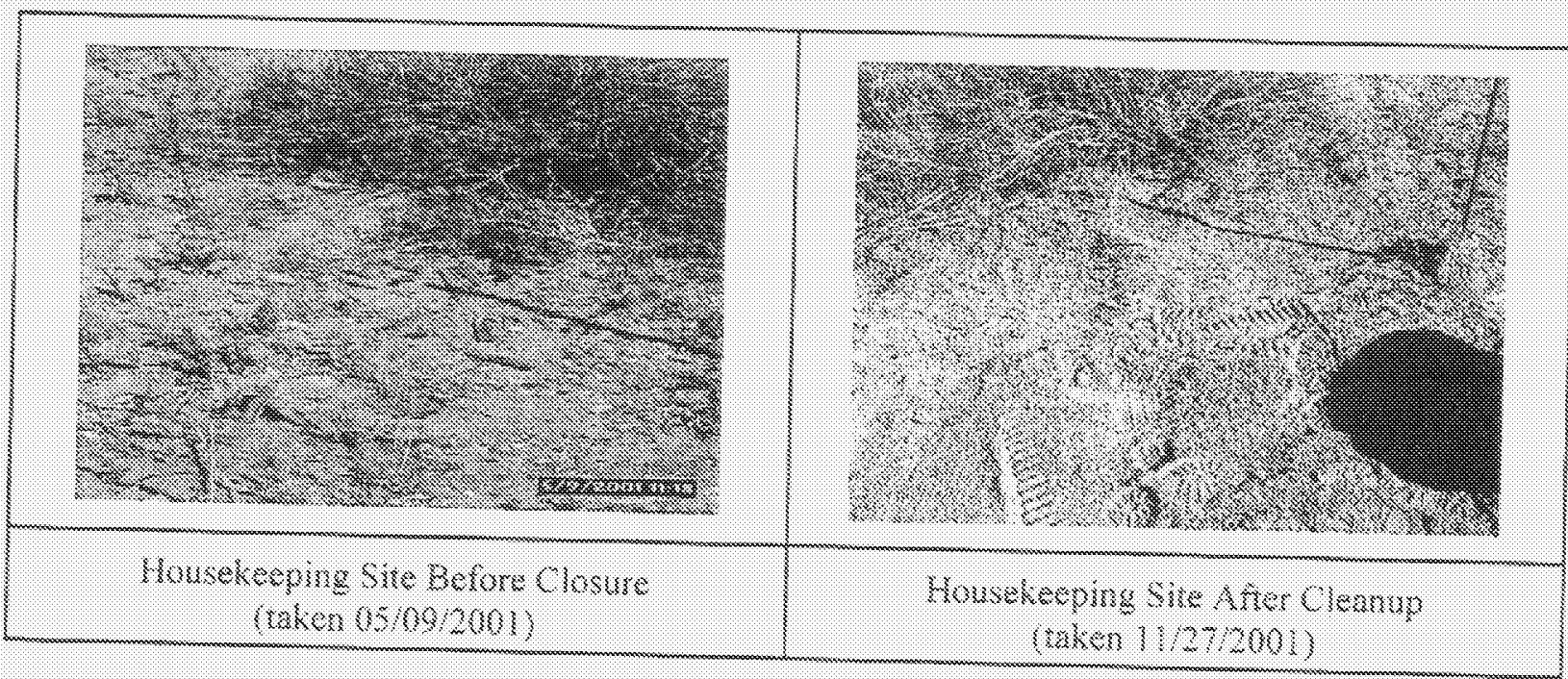

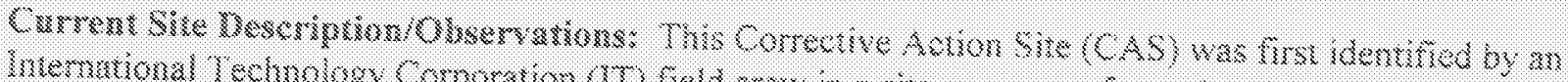

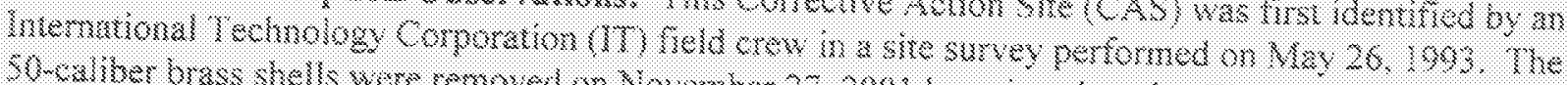

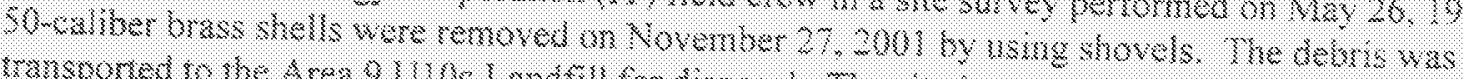

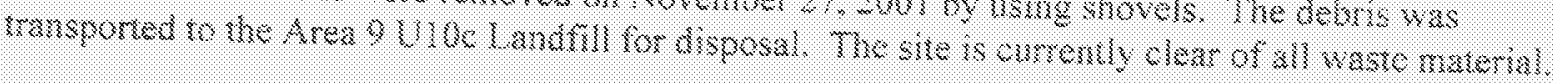

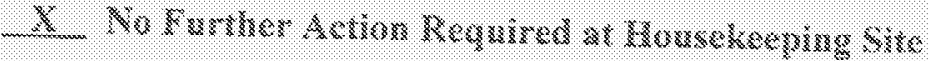

\begin{tabular}{|c|c|c|}
\hline 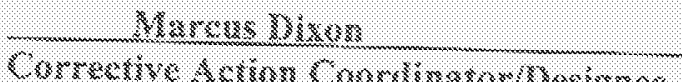 & 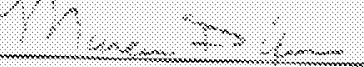 & $10.1: 3$ \\
\hline
\end{tabular}




\section{Sectored Housekeeping Sitc Closure Verification Form}

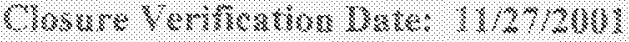

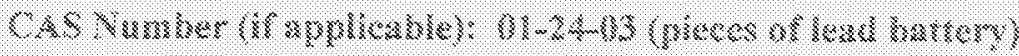

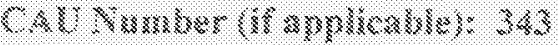

Sectse: Designatian: NTS

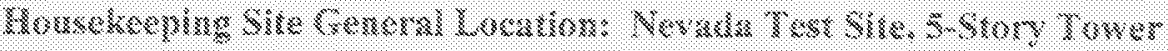

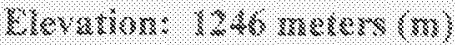

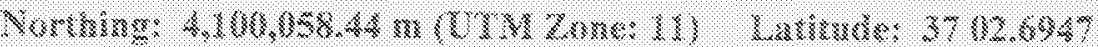

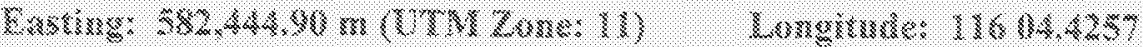

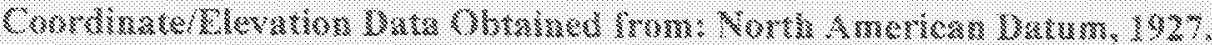

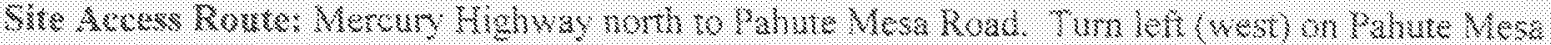

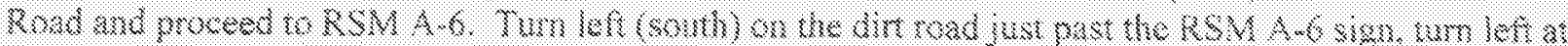

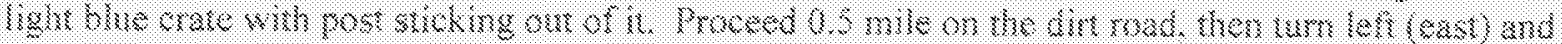

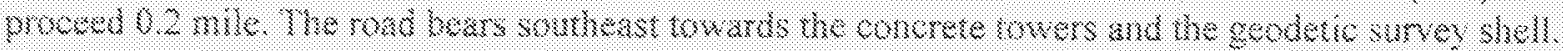

\begin{tabular}{|c|c|}
\hline 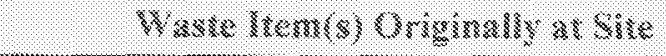 & 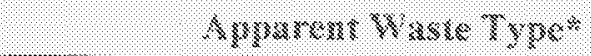 \\
\hline 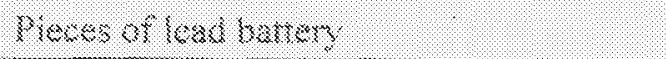 & 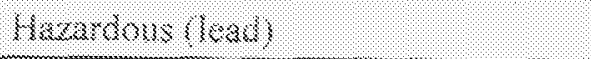 \\
\hline
\end{tabular}

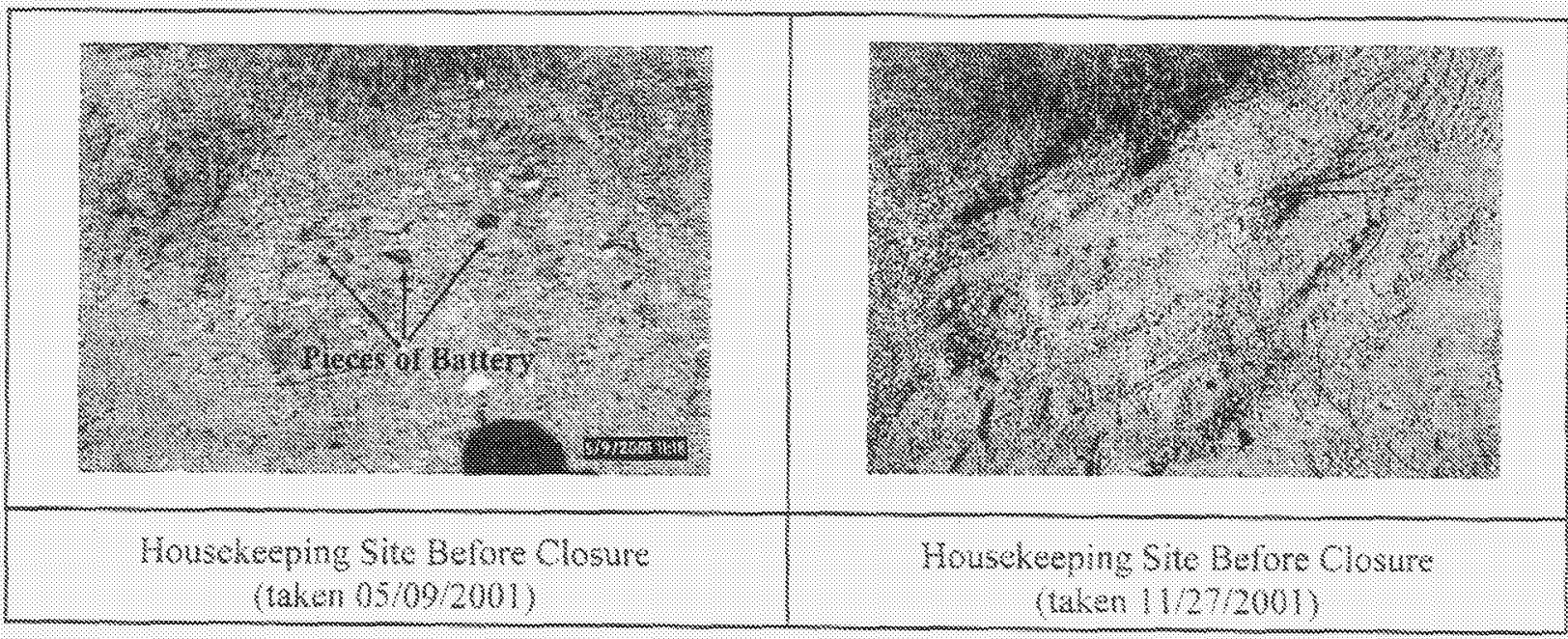

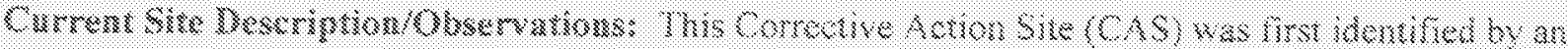

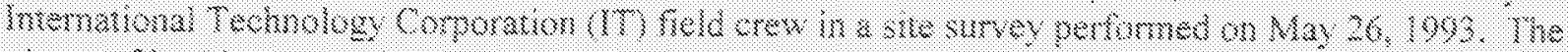

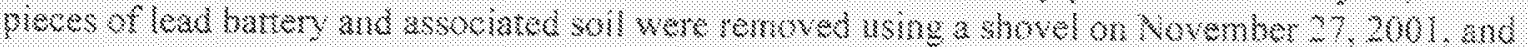

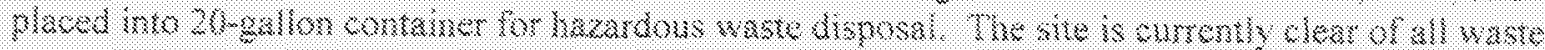

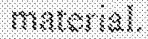

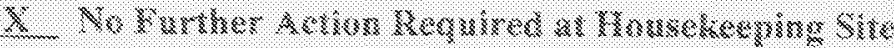

Mrorrexa Mixur:

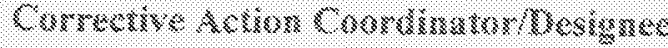

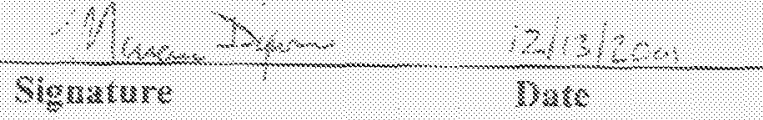

3.-2 


\section{Sectored Housekeeping Site Chosure Verification Form}

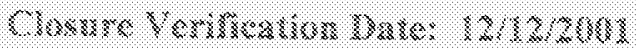

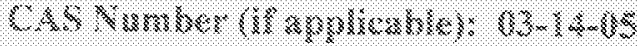

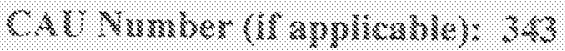

Secror Desigeszation: NrS

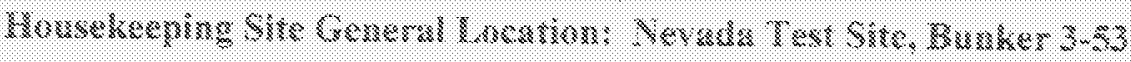

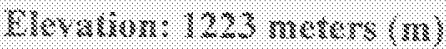

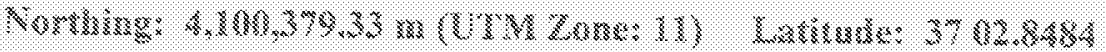

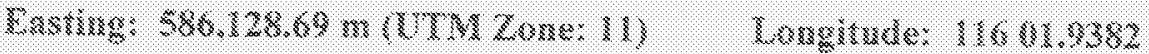

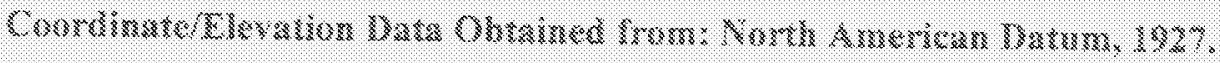

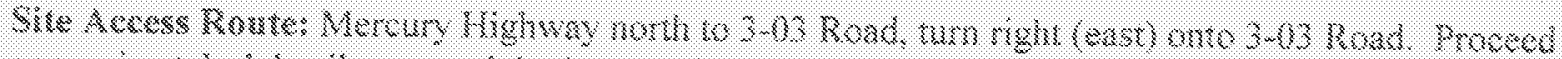

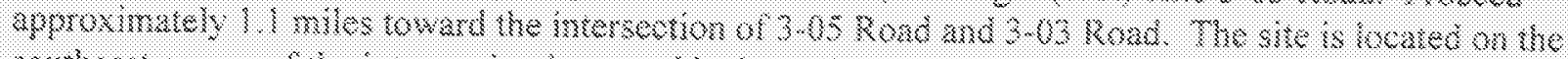

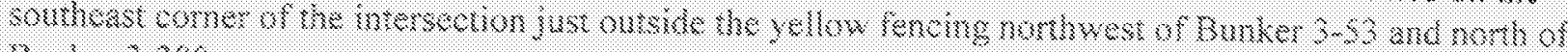

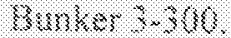

\begin{tabular}{|c|c|}
\hline 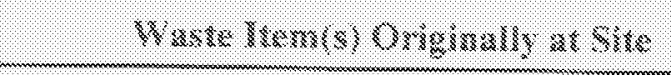 & 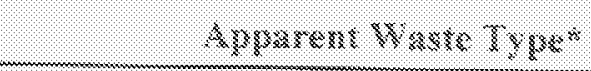 \\
\hline 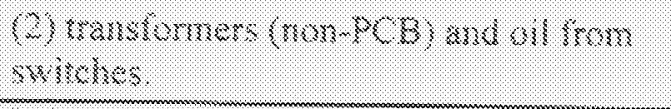 & Ordmary and serywahle \\
\hline
\end{tabular}

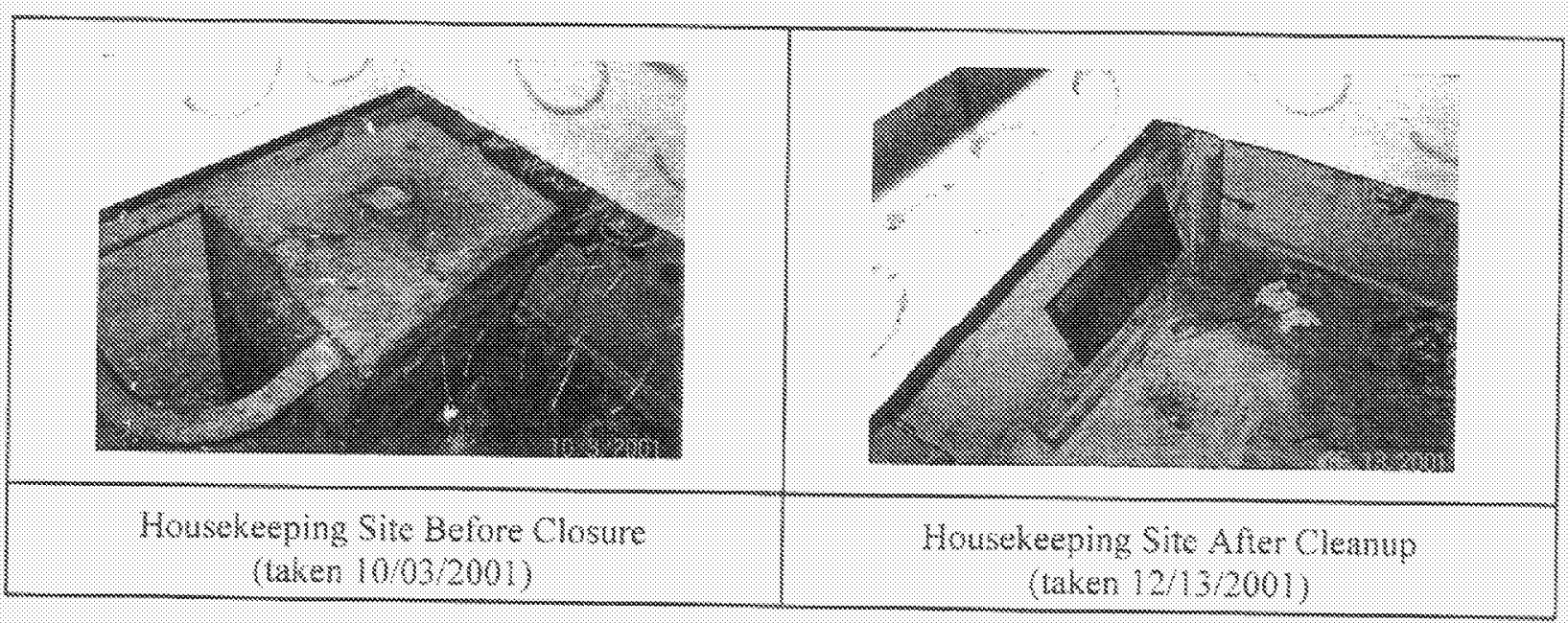

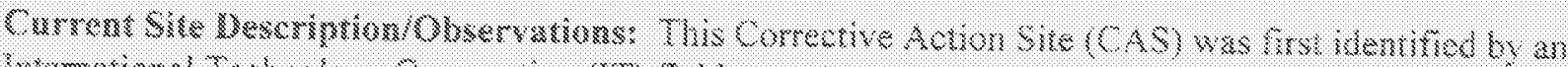

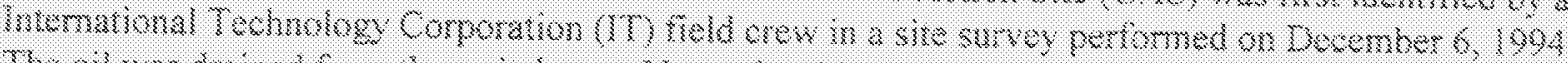

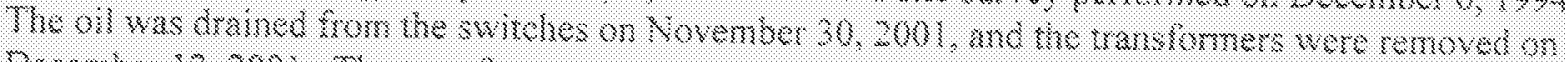

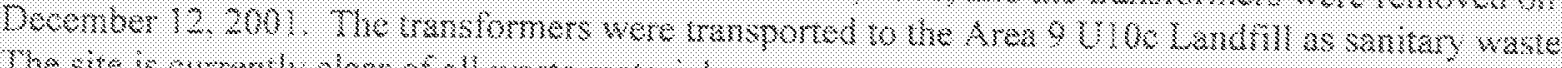
The site is wurrersty clear of all wate material.

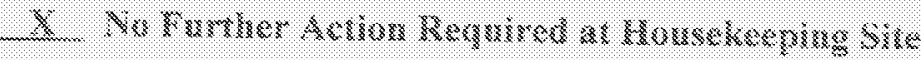

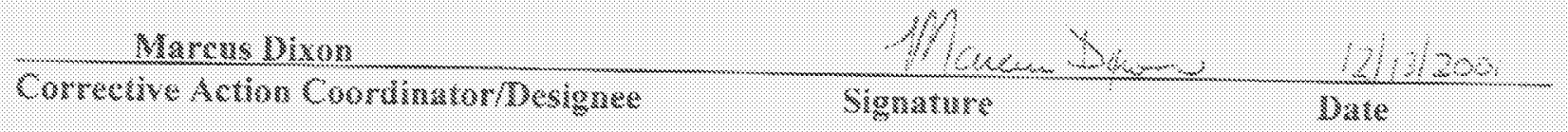




\section{Sectored Housekeeping Sile Clasure Verification Form}

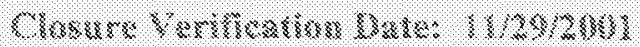

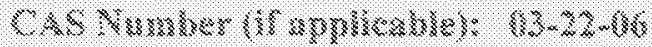

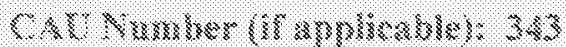

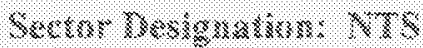

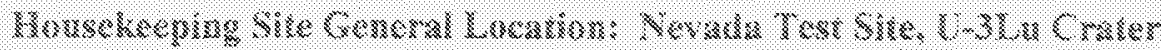

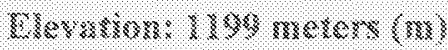

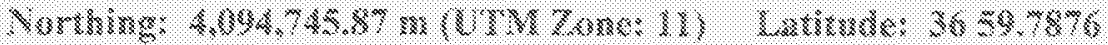

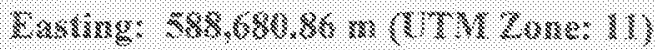

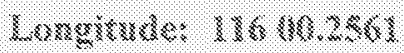

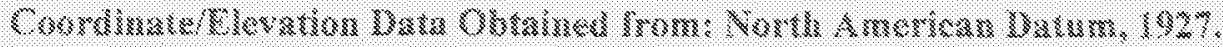

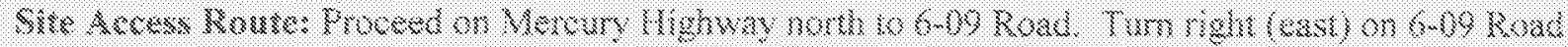

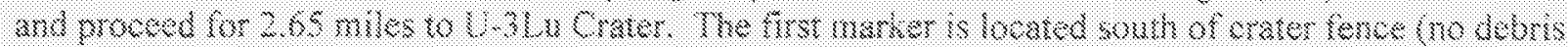

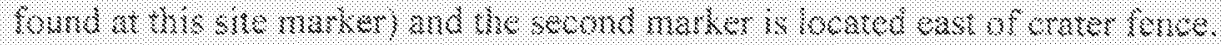

\begin{tabular}{|c|c|}
\hline 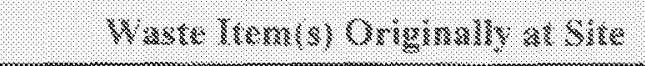 & Appartens: Waste Typa" \\
\hline Magengente & Ordimany \\
\hline
\end{tabular}

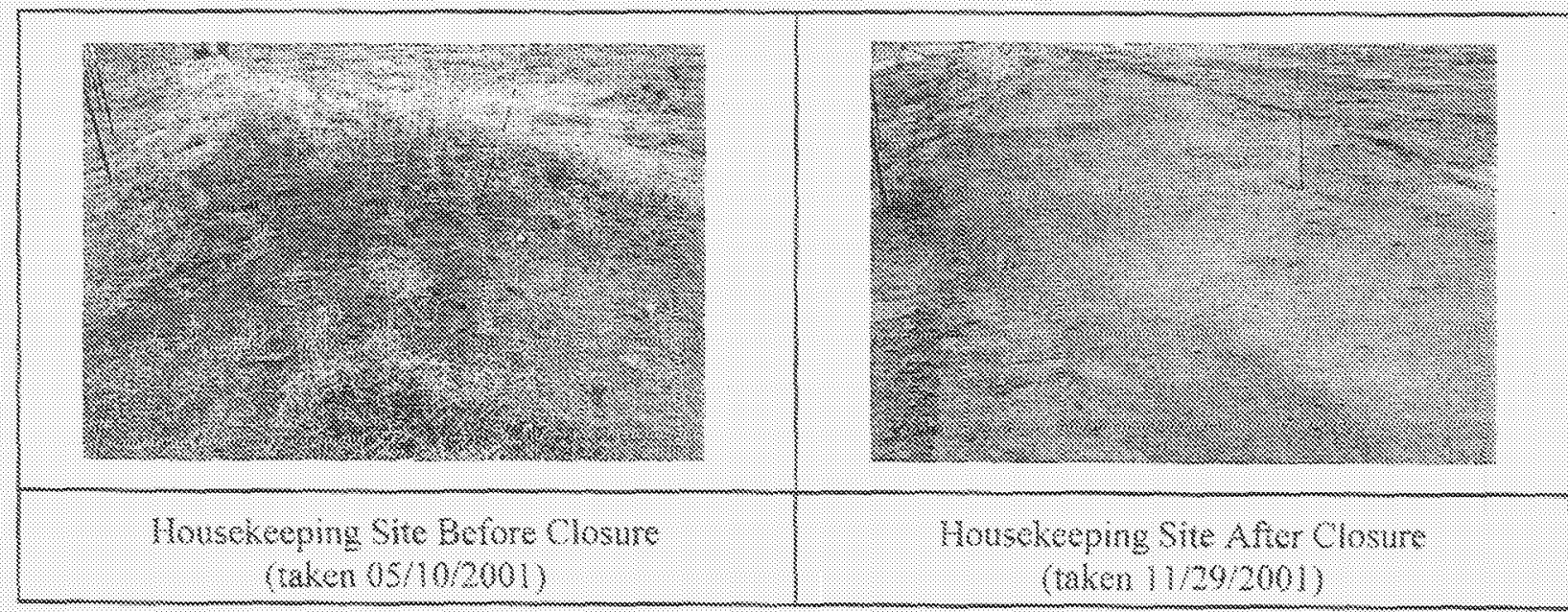

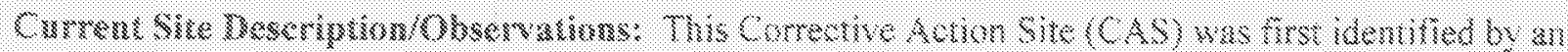

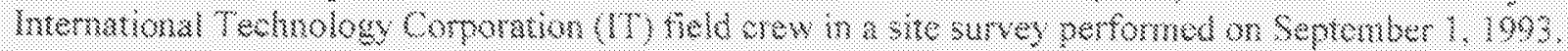

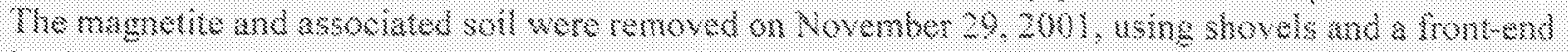

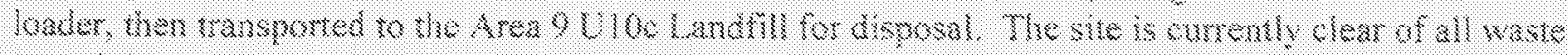
materia?.

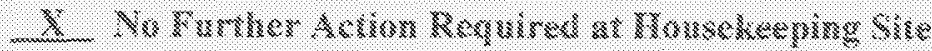

Marcas Dixan

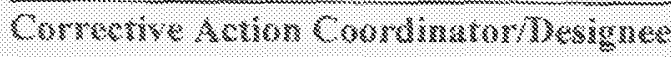

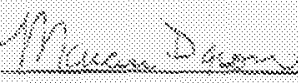

Signasures

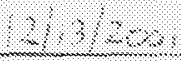

Yix 6 


\section{Sectored Housekeeping Site Closure Verification Form}

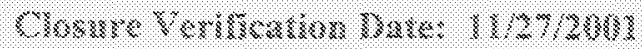

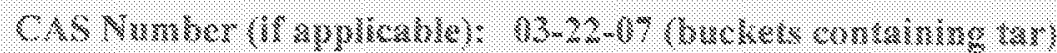

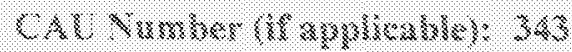

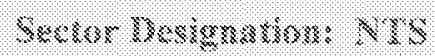

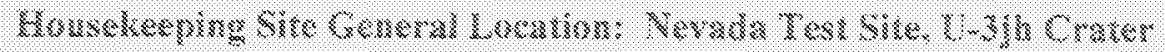

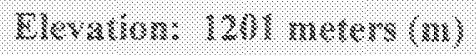

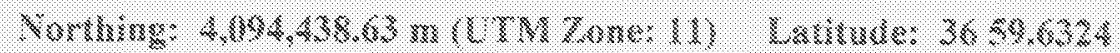

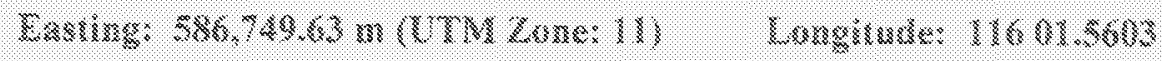

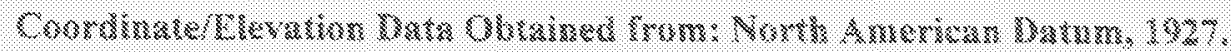

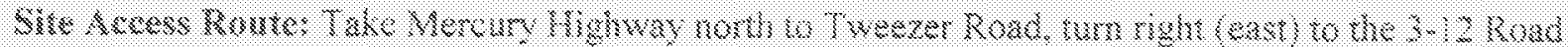

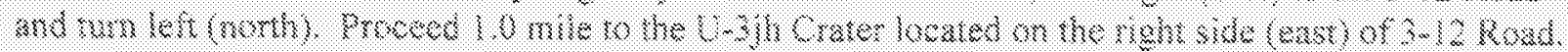

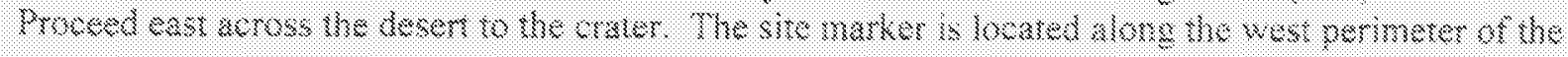
strater

\begin{tabular}{|c|c|}
\hline 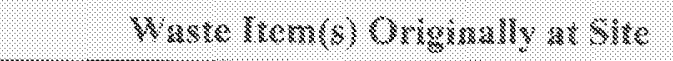 & 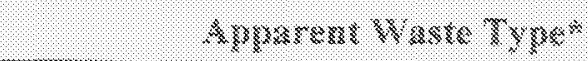 \\
\hline Metal buxckets commin my tay & 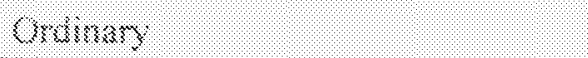 \\
\hline
\end{tabular}

\begin{tabular}{|c|c|}
\hline 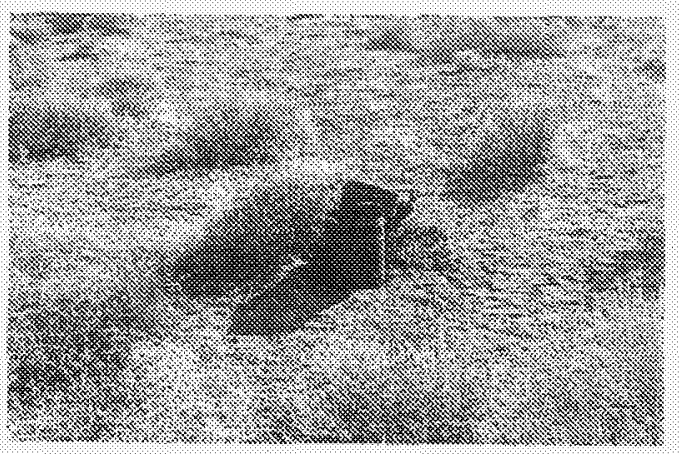 & 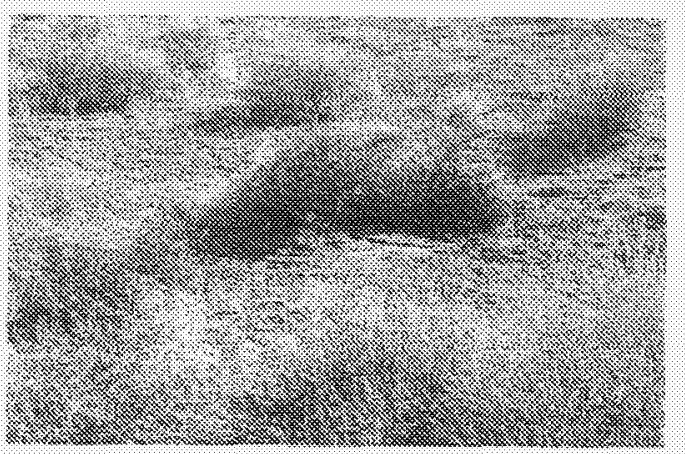 \\
\hline 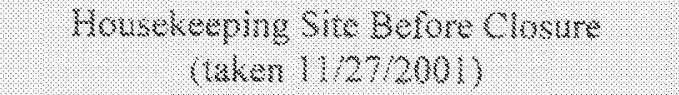 & 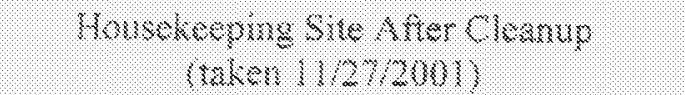 \\
\hline
\end{tabular}

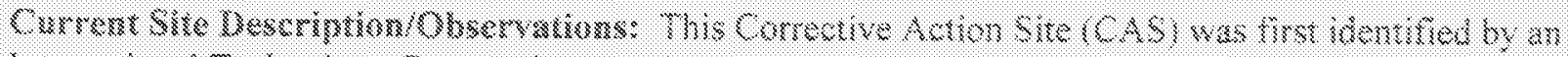

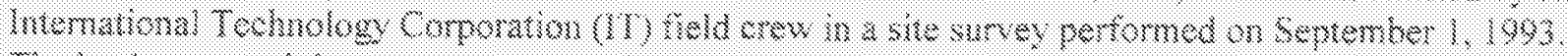

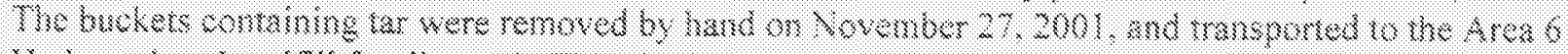

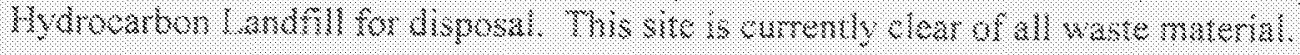

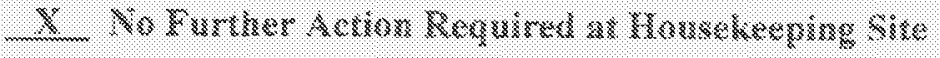

Marcus Dixon

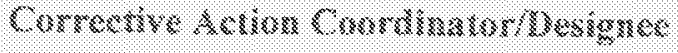

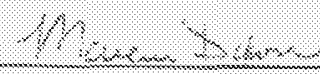

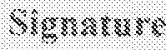

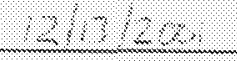

$0 \times 2 \times 2$ 


\section{Sectored Housekeping Site Closure Verification Form}

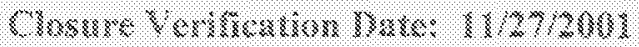

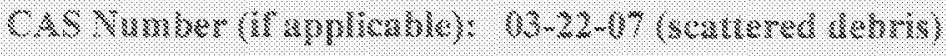

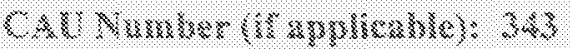

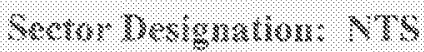

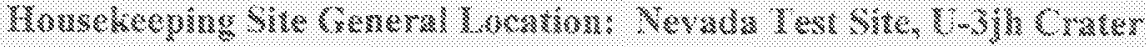

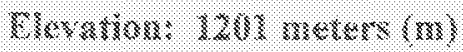

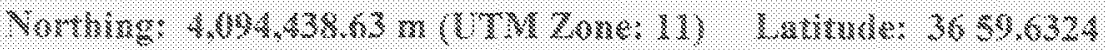

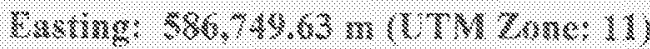

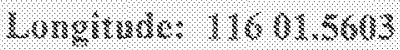

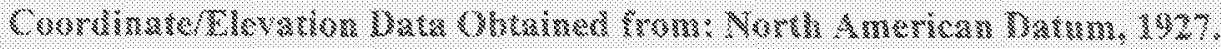

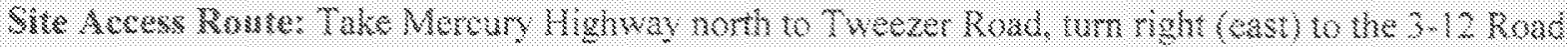

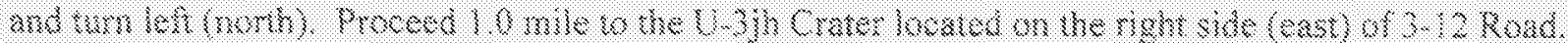

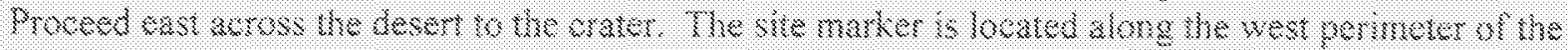
irkxter.

\begin{tabular}{|c|c|}
\hline 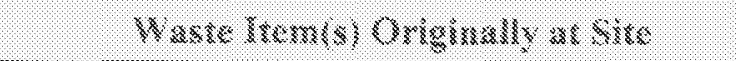 & 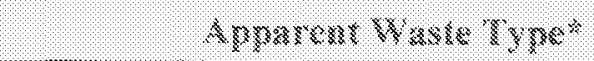 \\
\hline 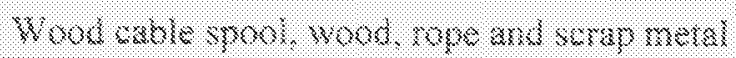 & 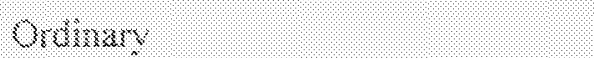 \\
\hline
\end{tabular}

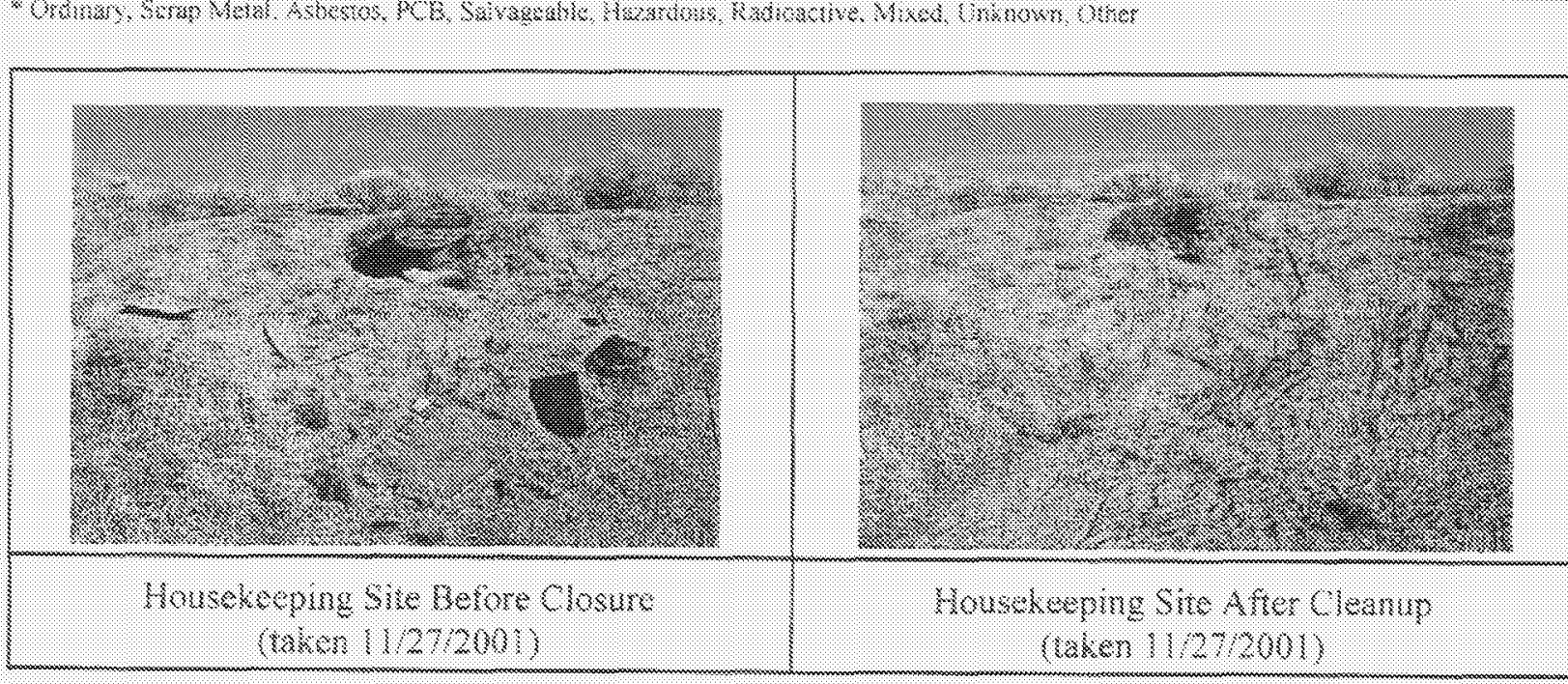

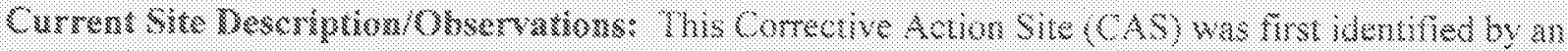

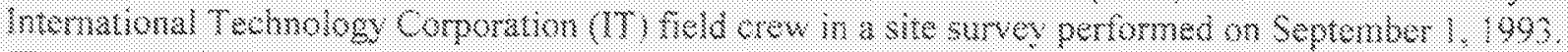

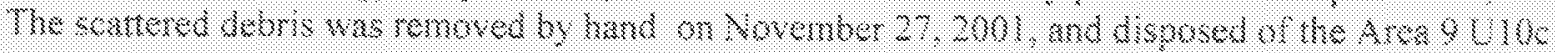

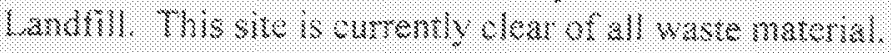

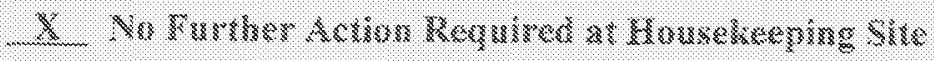

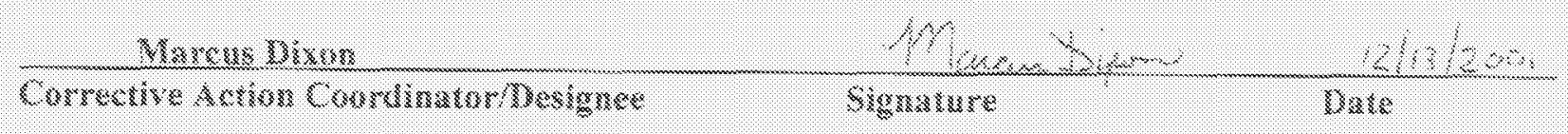




\section{Sectored Housekeeping Site Clasure Verification Form}

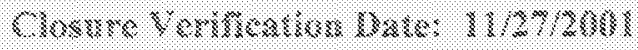

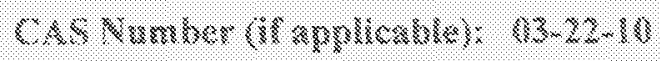

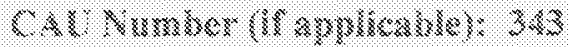

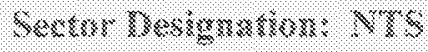

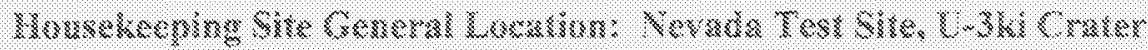

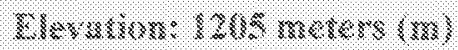

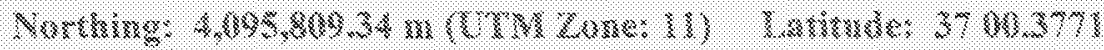

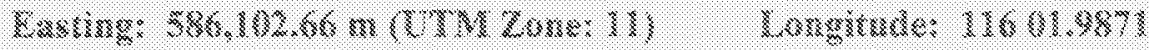

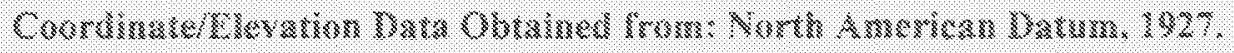

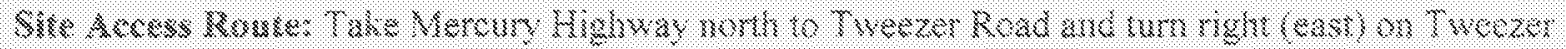

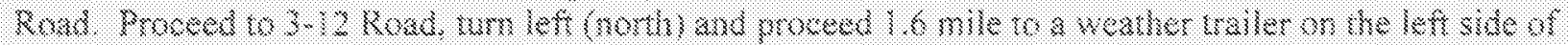

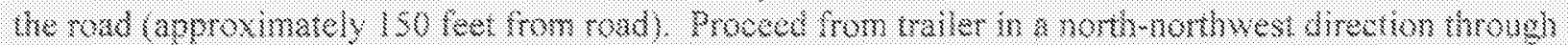

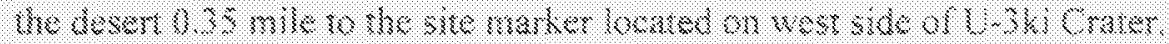

\begin{tabular}{|c|c|}
\hline 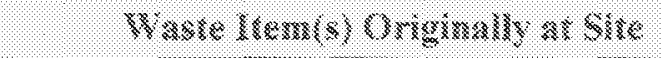 & 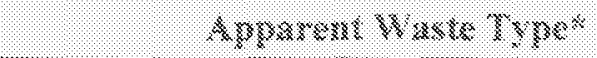 \\
\hline 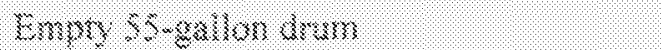 & 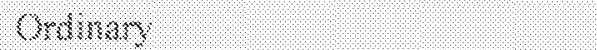 \\
\hline
\end{tabular}

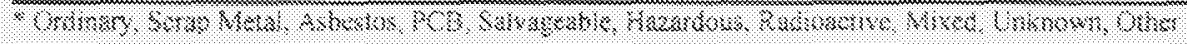

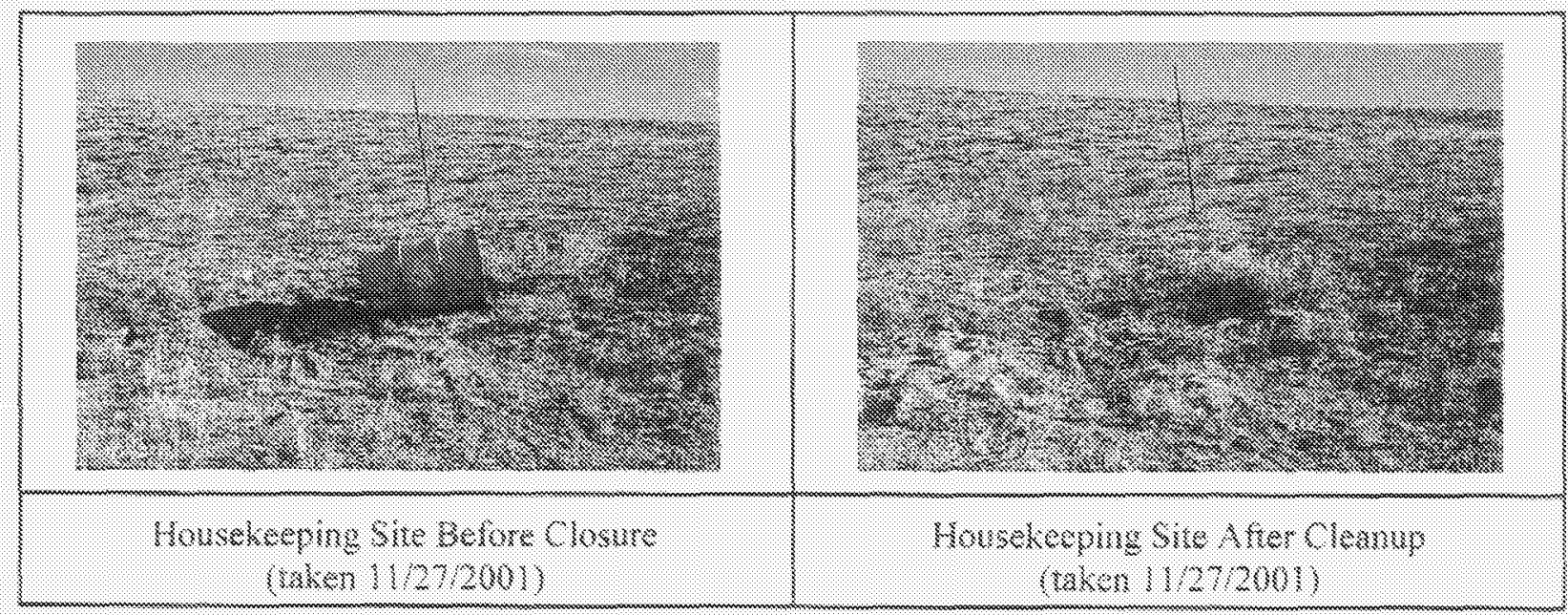

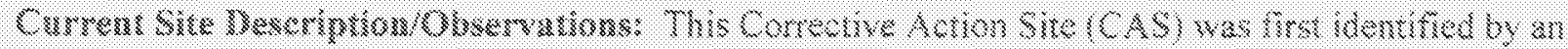

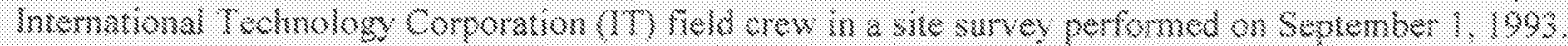

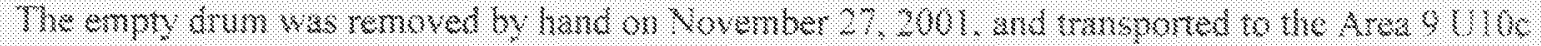
Landill for disposal. This sites is comrenty olenr of all waste material.

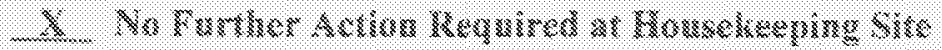

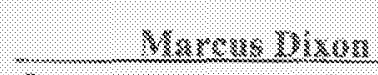

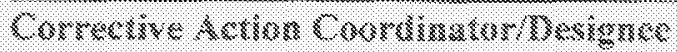

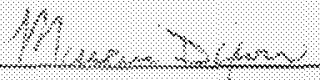

Sigrs:arsse

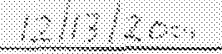

\section{Pro: $\{10$}




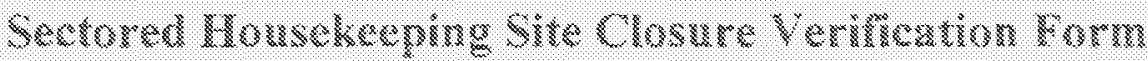

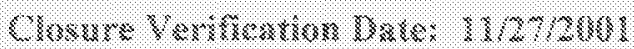

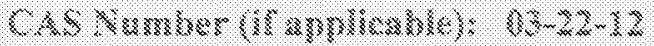

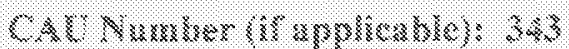

Sector: Dexigratuion: VTS

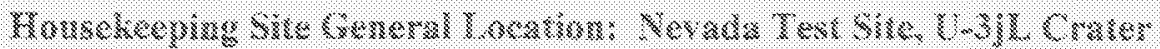

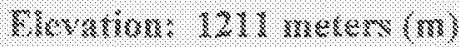

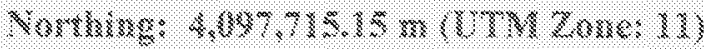

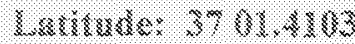

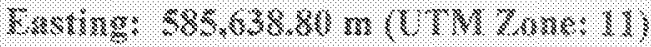

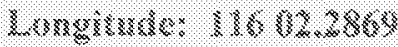

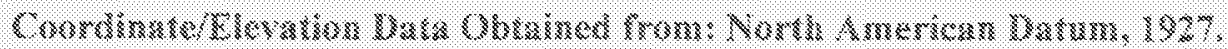

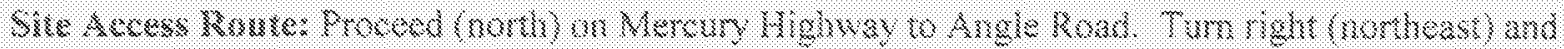

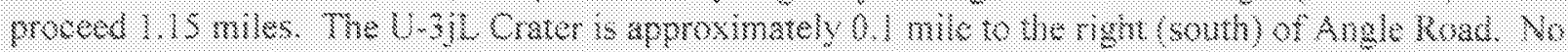

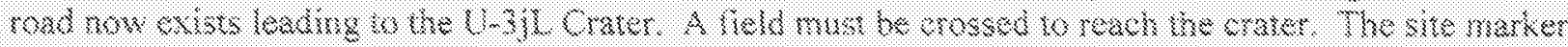
is lacateri on the west side of the crater.

\begin{tabular}{|c|c|}
\hline 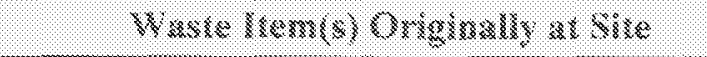 & 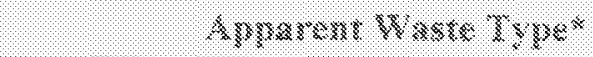 \\
\hline 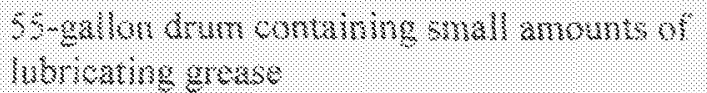 & (2)xilisery \\
\hline
\end{tabular}

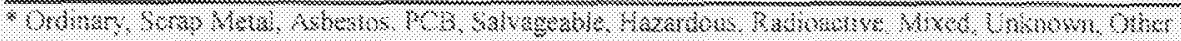

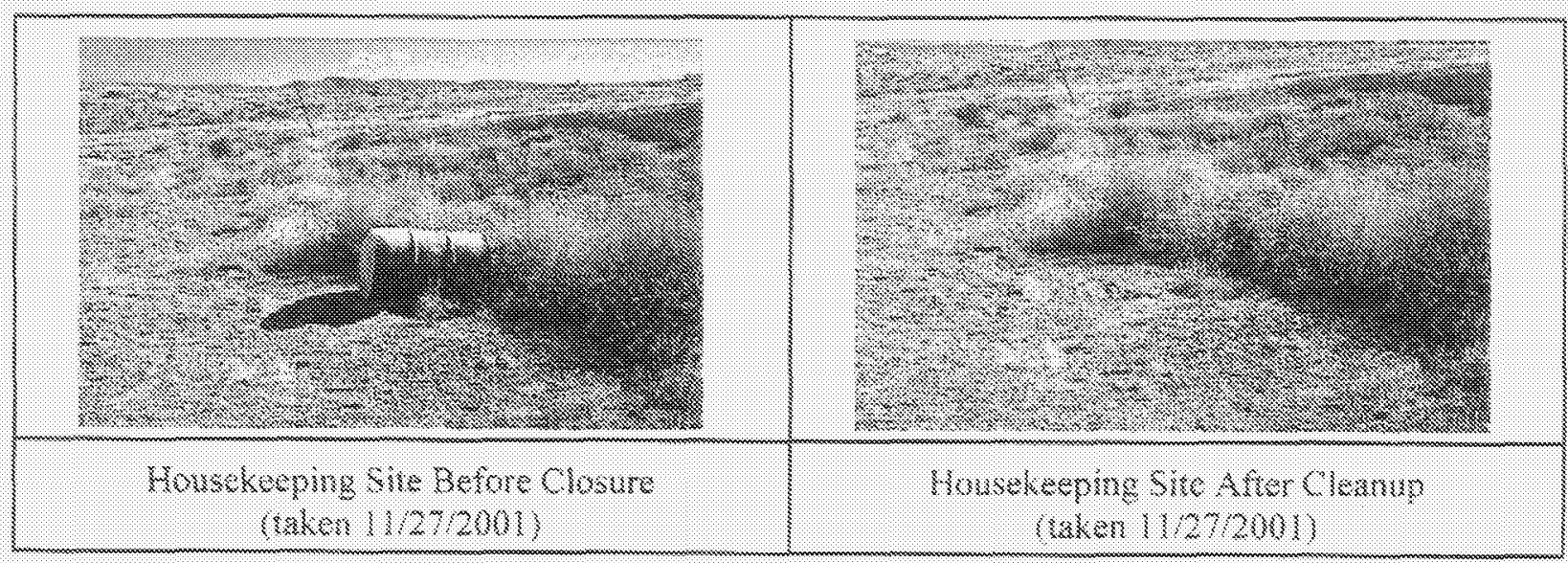

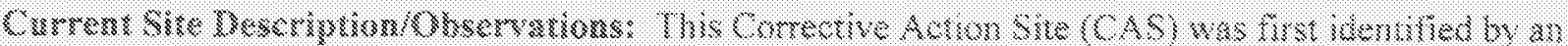

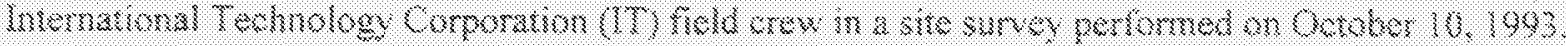

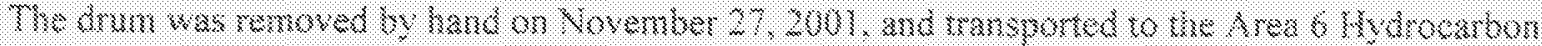

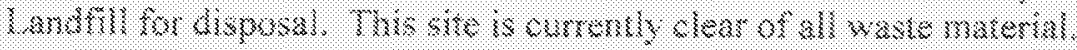

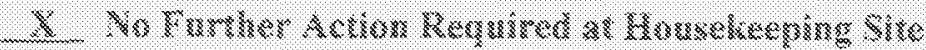

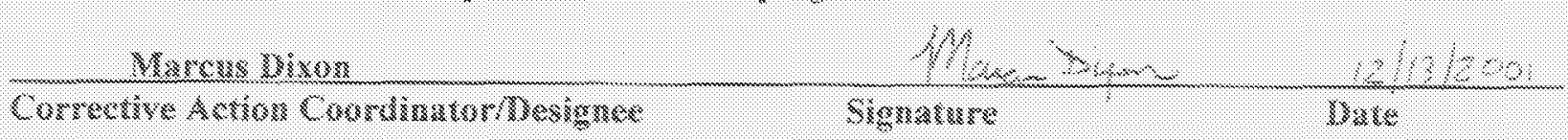




\section{Sectored Housekeeping Site Closure Verification Form}

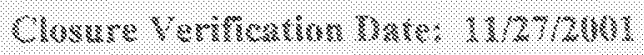

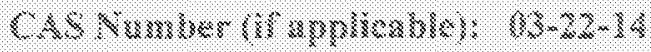

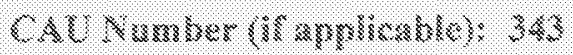

Sostor $D$ sos

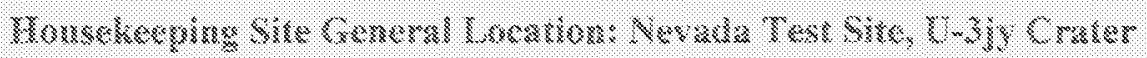

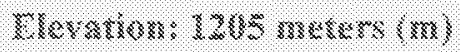

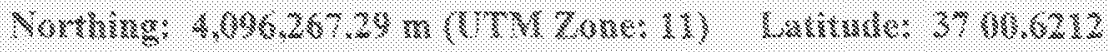

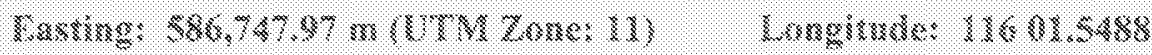

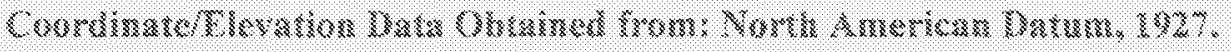

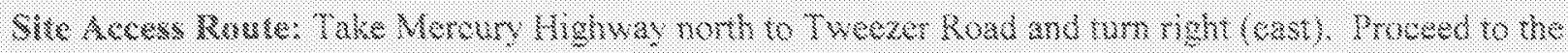

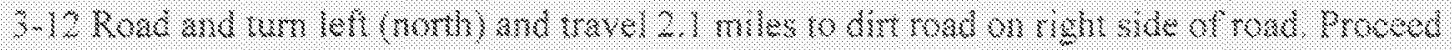

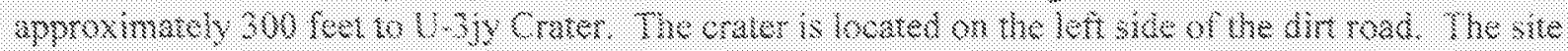

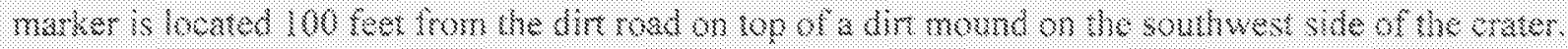

\begin{tabular}{|c|c|}
\hline 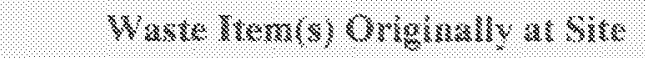 & 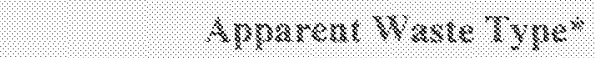 \\
\hline 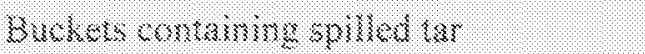 & 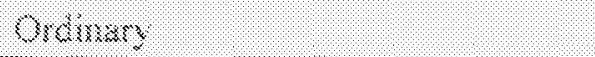 \\
\hline
\end{tabular}

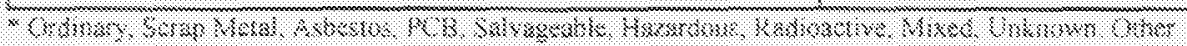

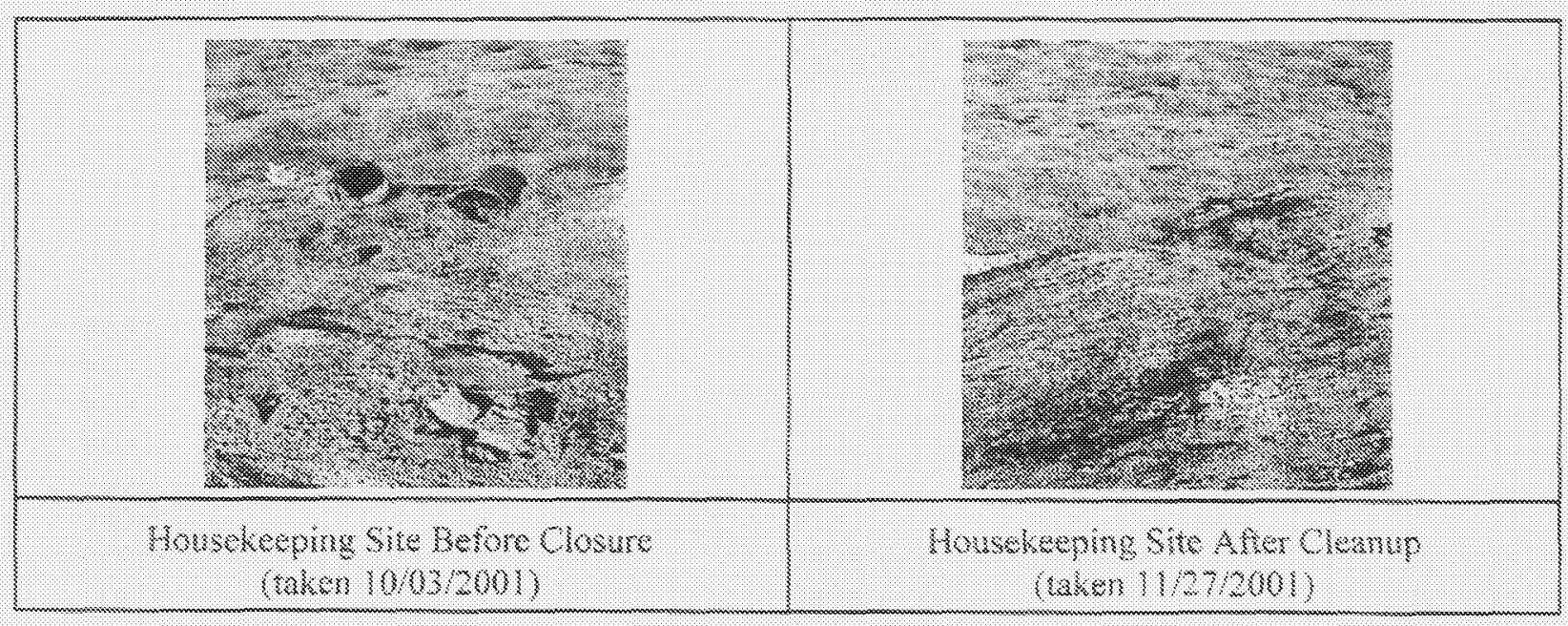

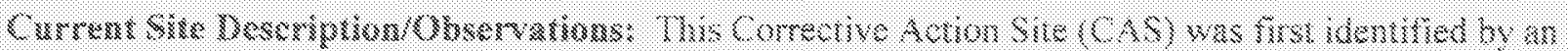

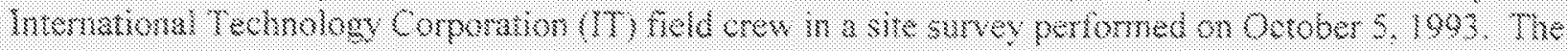

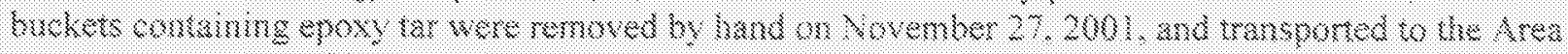

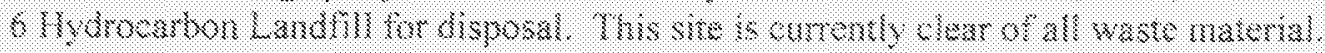

\section{Vis Frm}

Marreur Uixen

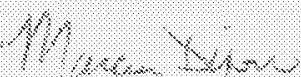

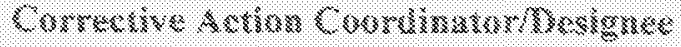

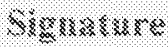
$1.2 .10 .10 \%$ T) 


\title{
Sectored Housekeeping Site Closurc Verification Form
}

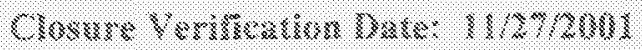

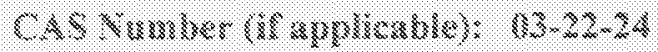

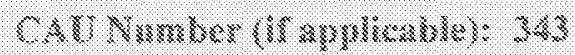

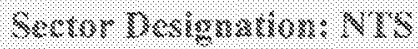

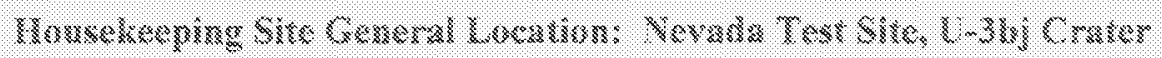

2)

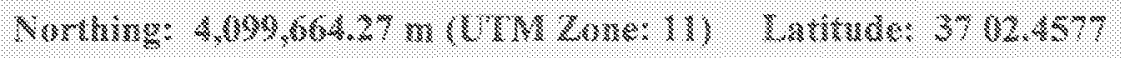

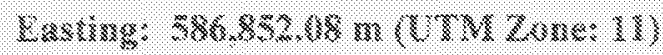

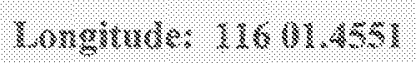

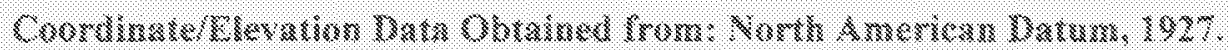

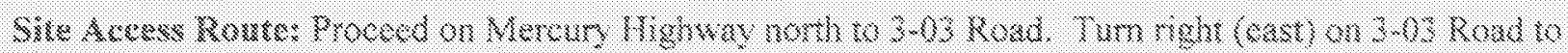
3-35 Kuad. Tum rigkt (

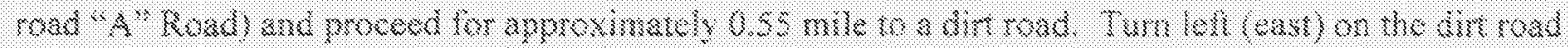

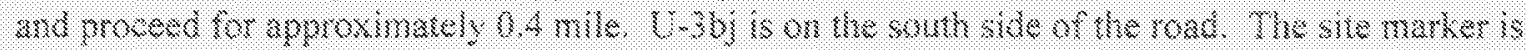

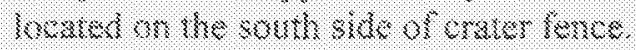

\begin{tabular}{|c|c|}
\hline 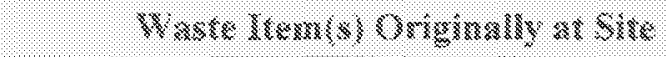 & Applaseren: Waste TYpre \\
\hline 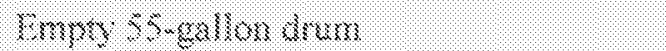 & 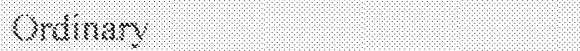 \\
\hline
\end{tabular}

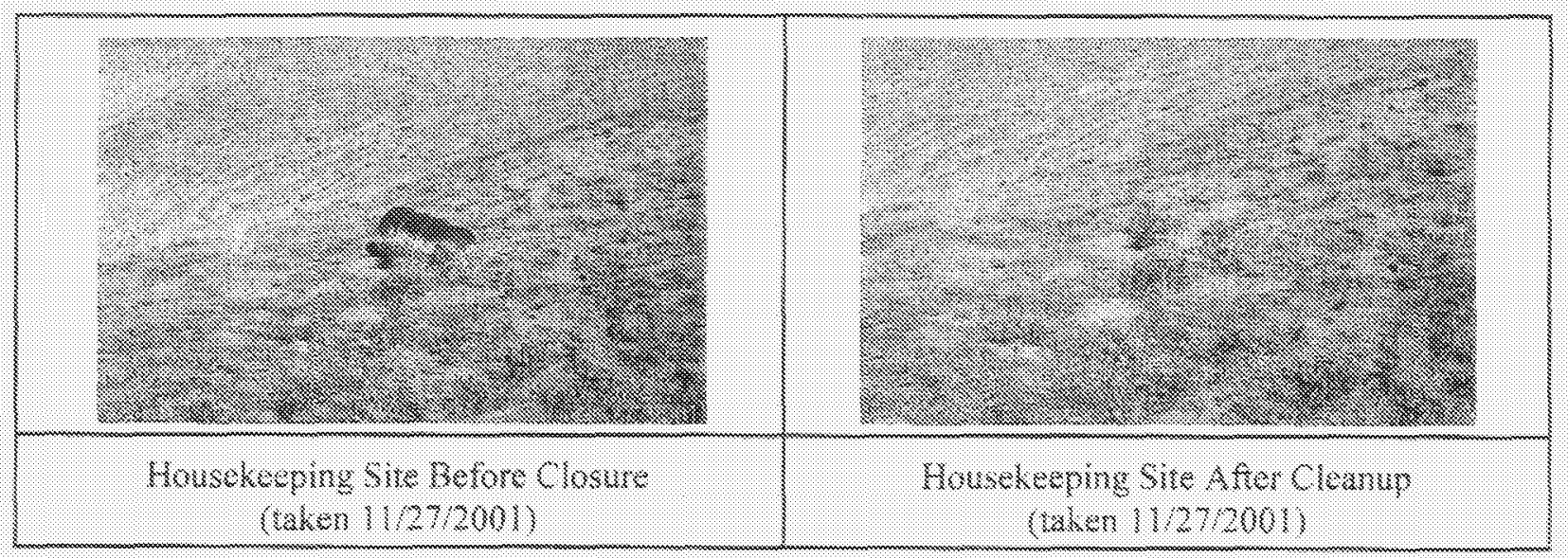

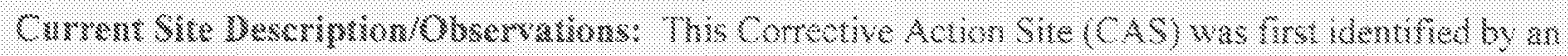

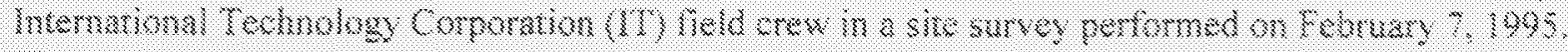

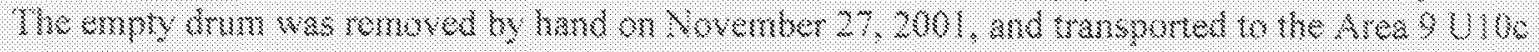

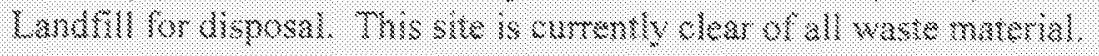

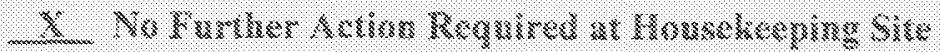

Mixrous Dirou

Corrective: Aerion Conrdinatom Dexignese

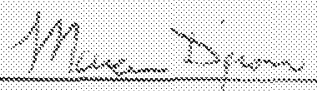

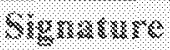

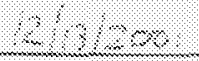

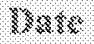




\section{Sectored Housekecping Site Clssure Verification Form}

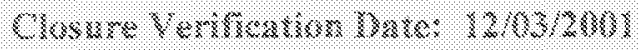

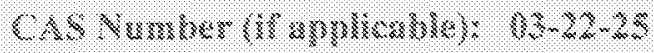

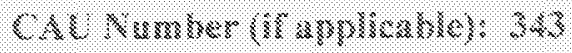

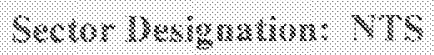

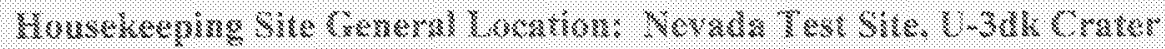

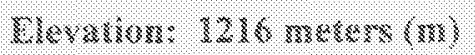

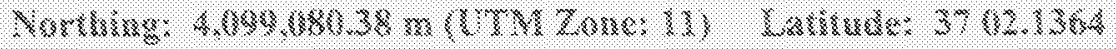

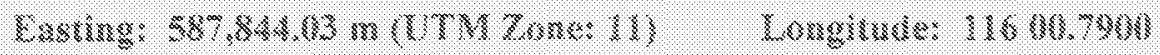

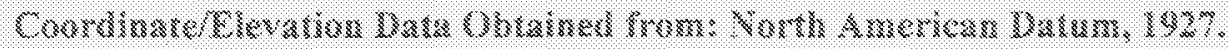

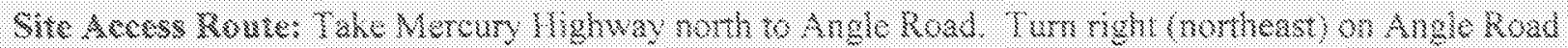

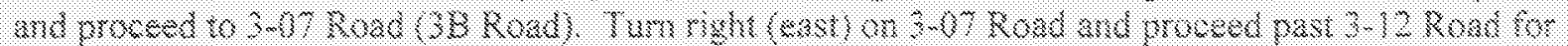

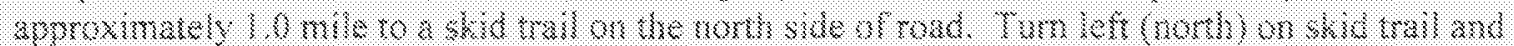

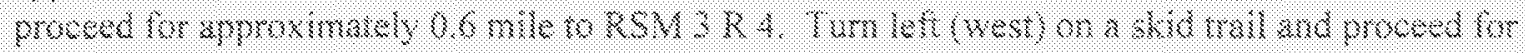

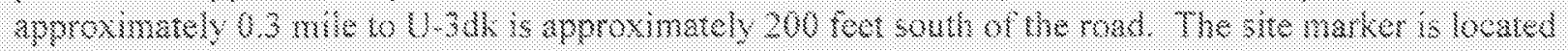

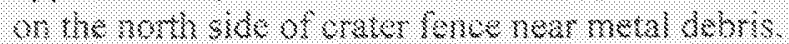

\begin{tabular}{|c|c|}
\hline 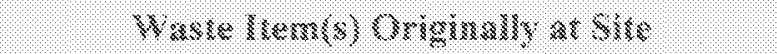 & 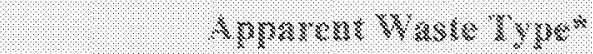 \\
\hline 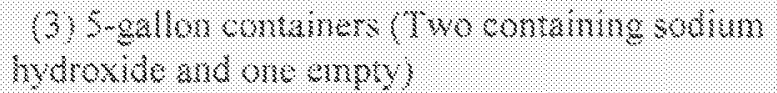 & 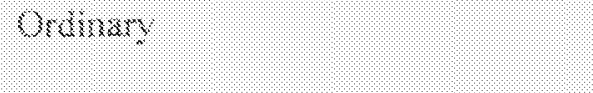 \\
\hline
\end{tabular}

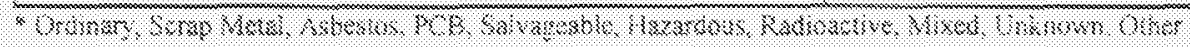

\begin{tabular}{|c|c|}
\hline 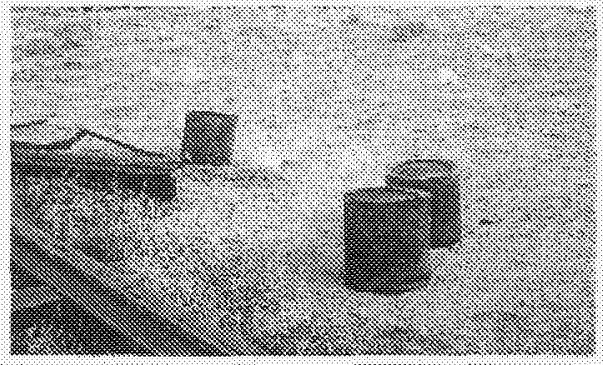 & 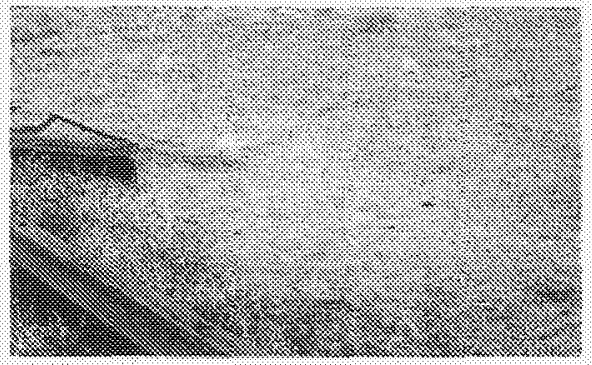 \\
\hline 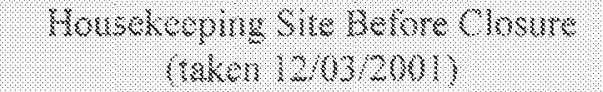 & 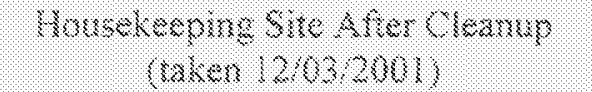 \\
\hline
\end{tabular}

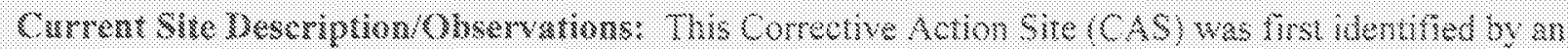

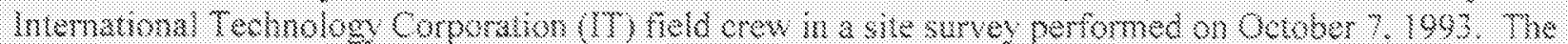

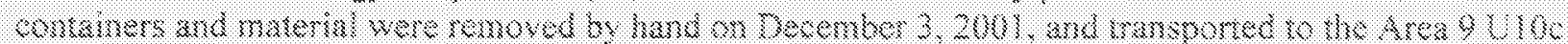

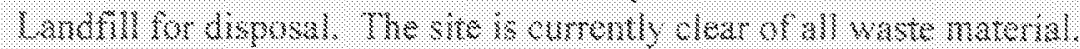

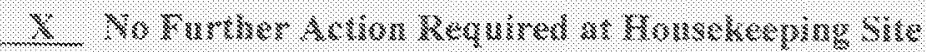

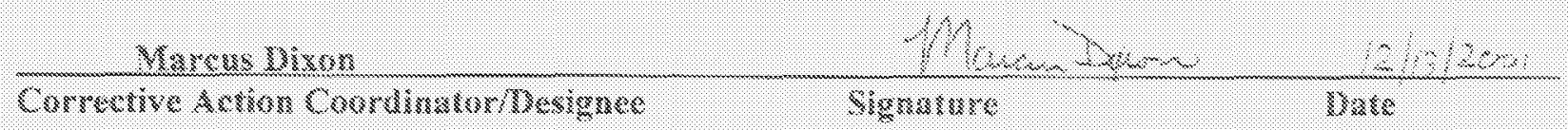




\section{Sectored Housekeeping Site Closure Verifieation Form}

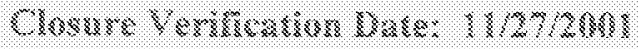

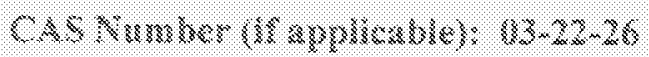

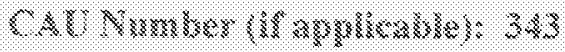

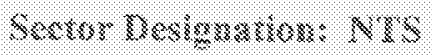

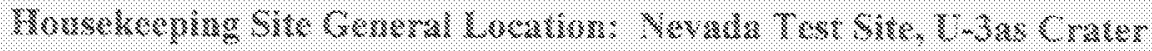

Exevation: 1222 meters (m)

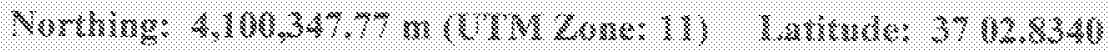

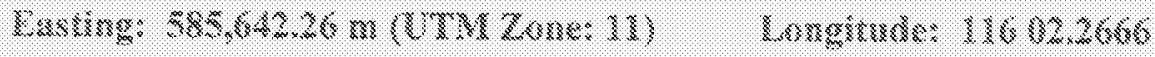

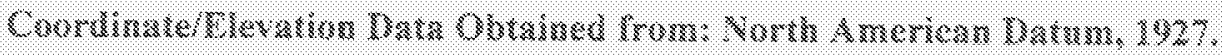

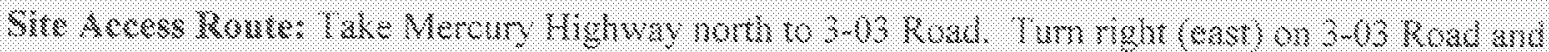

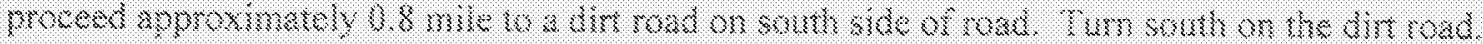

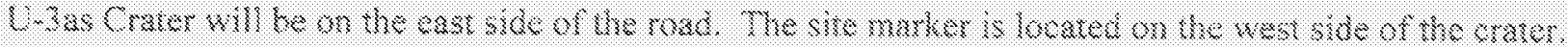

\begin{tabular}{|c|c|}
\hline Waste lenzuss Oriminuliy at Sike & 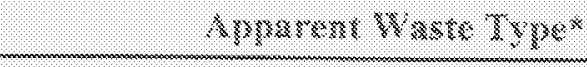 \\
\hline 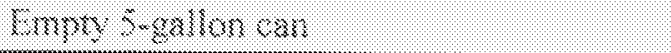 & Uruimarars \\
\hline
\end{tabular}

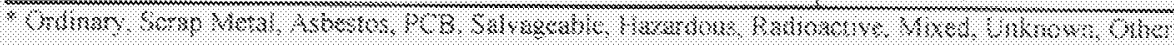

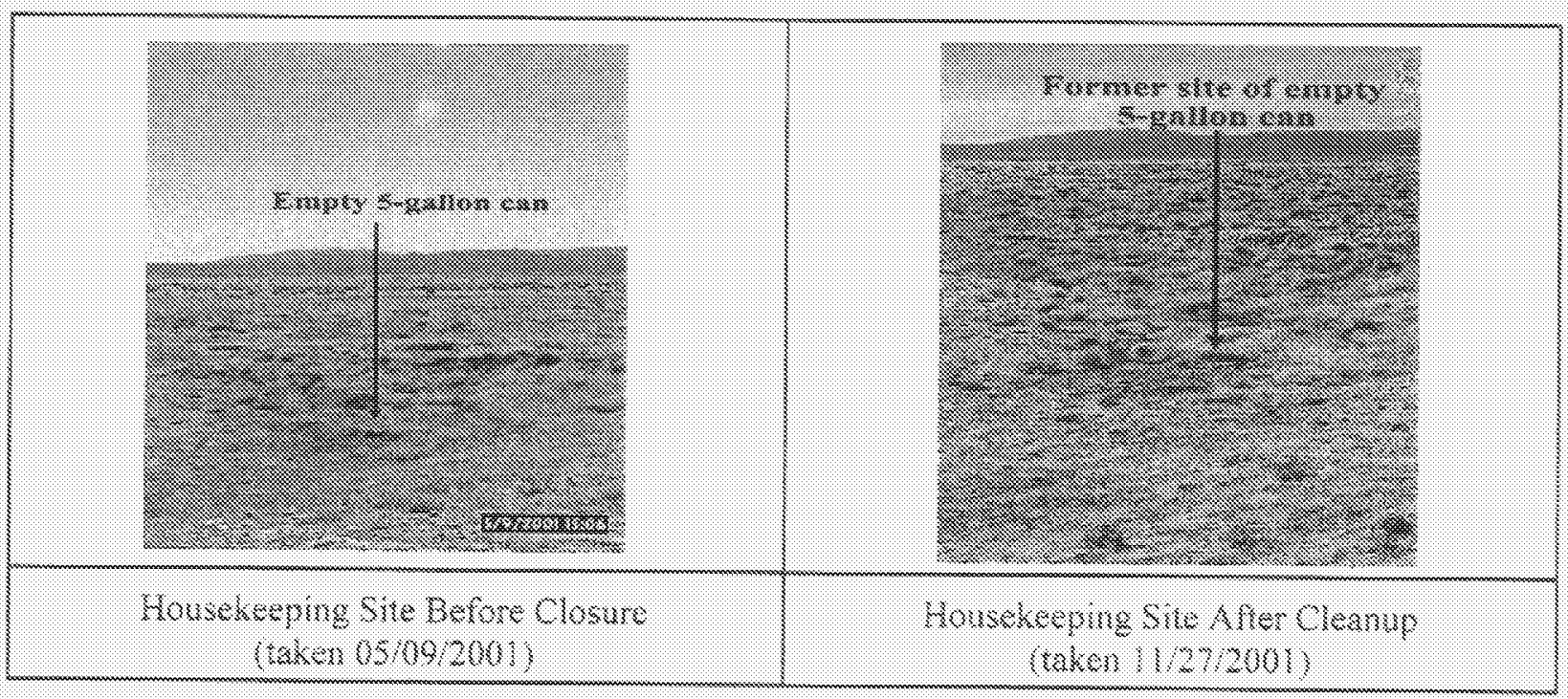

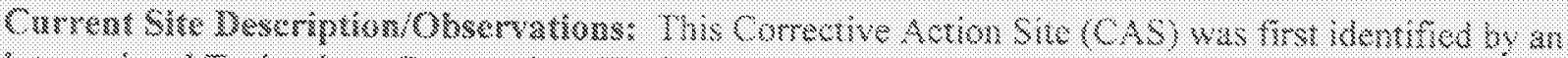

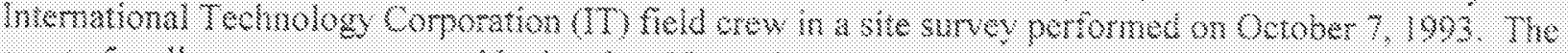

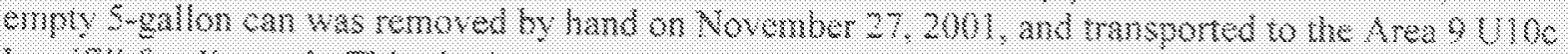

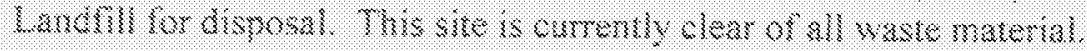

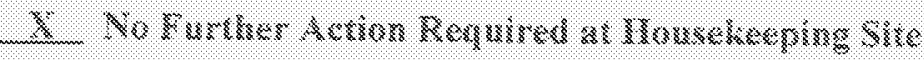

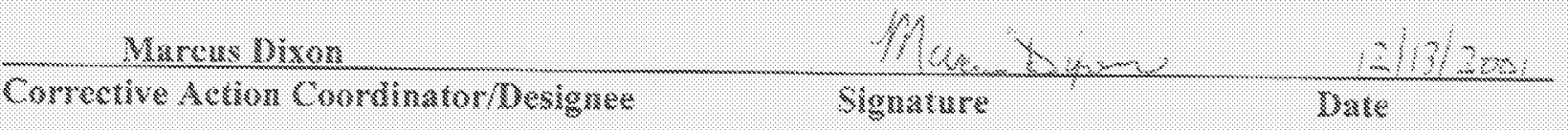




\section{Sectored Housekeeping Site Closure Verification Form}

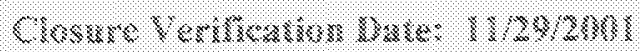

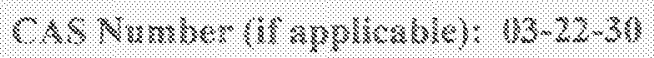

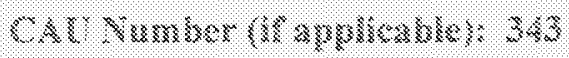

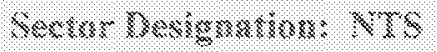

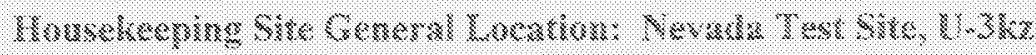

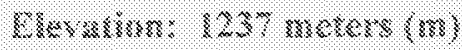

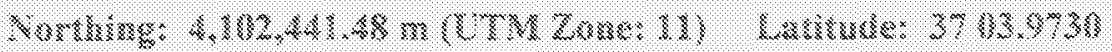

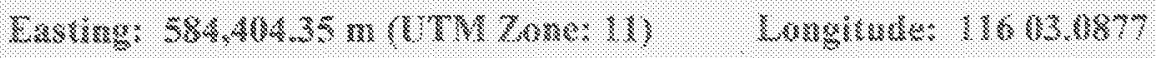

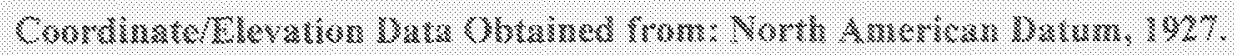

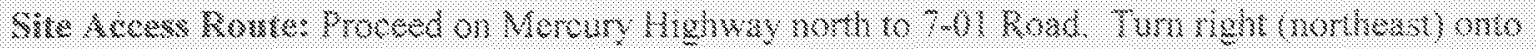

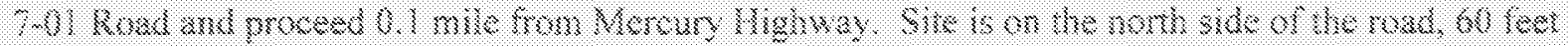

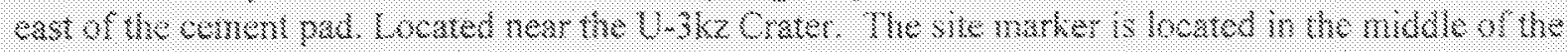

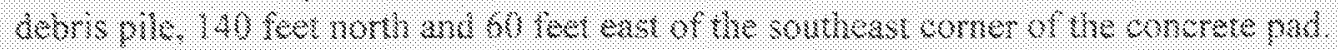

\begin{tabular}{|c|c|}
\hline 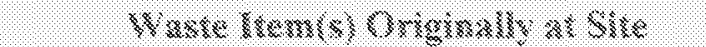 & A \\
\hline 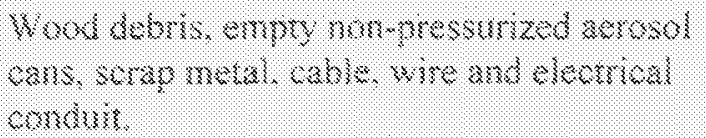 & 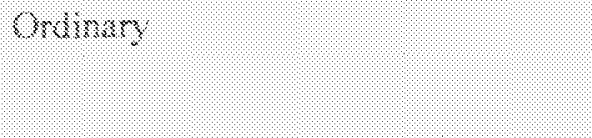 \\
\hline
\end{tabular}

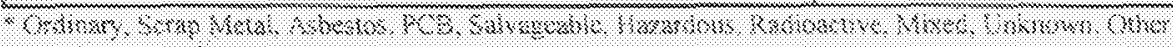

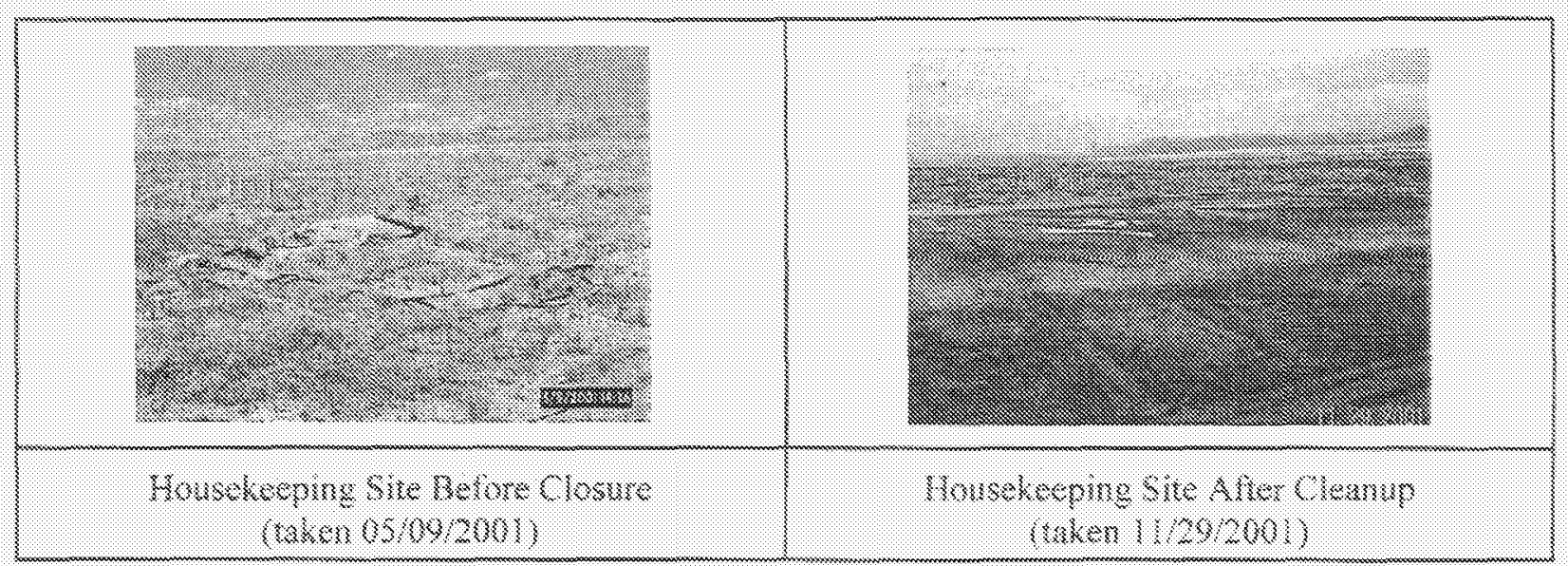

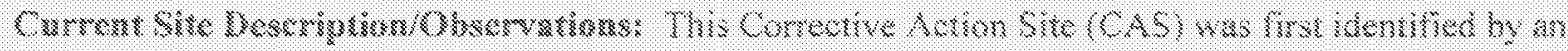

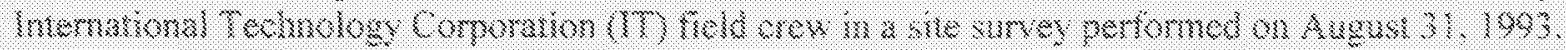

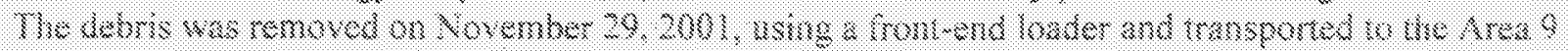

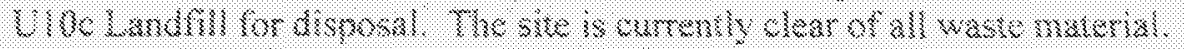

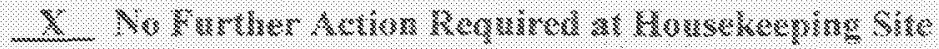

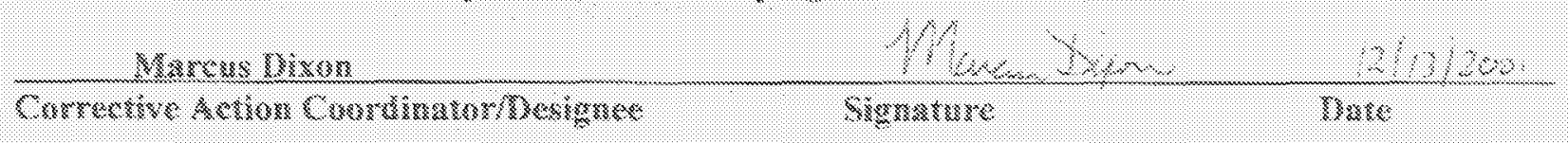




\section{Sectored Housekeeping Site Closure Verification Form}

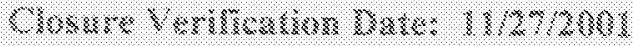

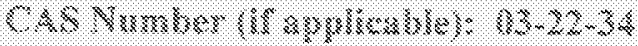

CAU Namber (W applicabic): 343

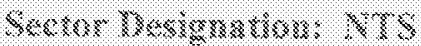

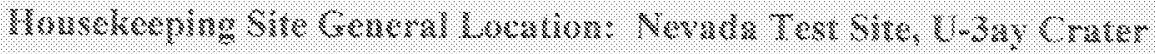

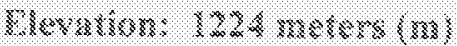

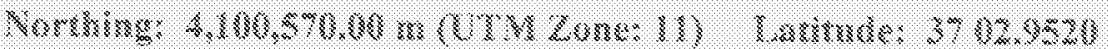

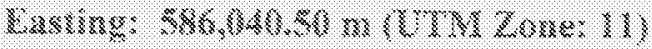

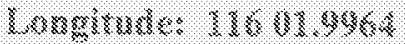

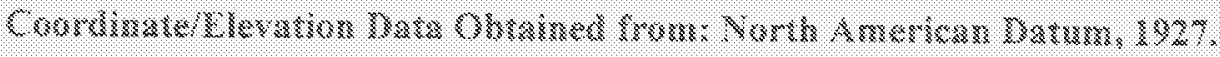

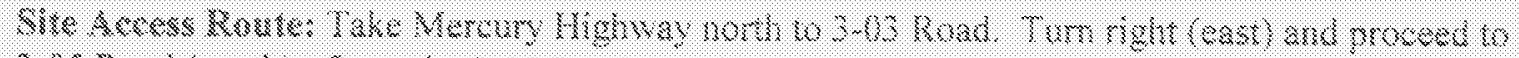

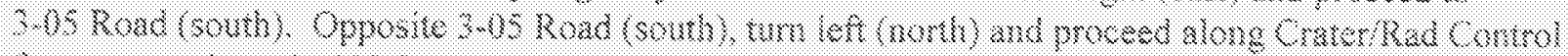

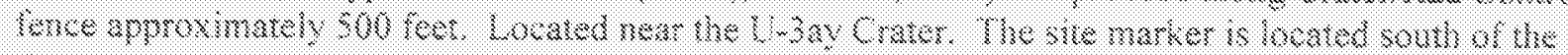

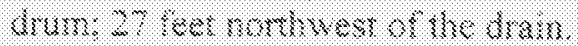

\begin{tabular}{|c|c|}
\hline 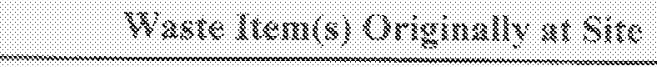 & 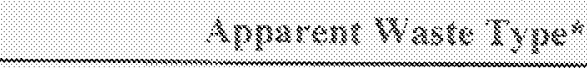 \\
\hline 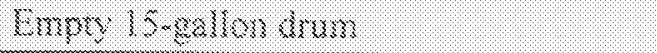 & Oruzisuars? \\
\hline
\end{tabular}

\begin{tabular}{|c|c|}
\hline 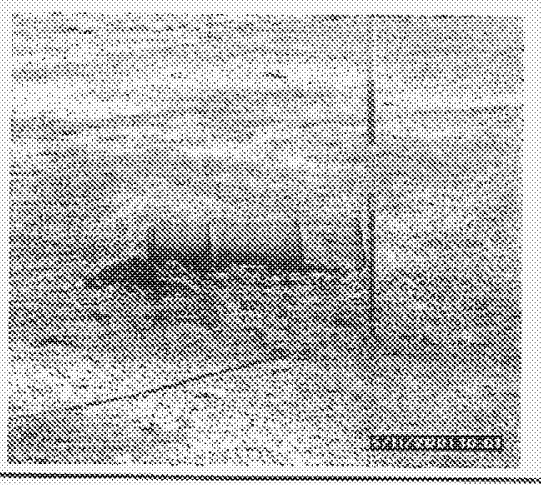 & 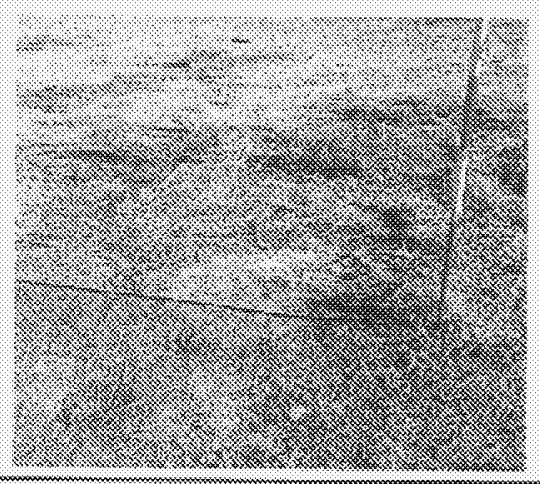 \\
\hline 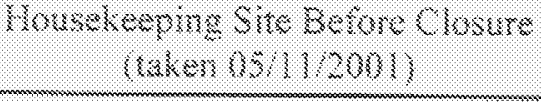 & 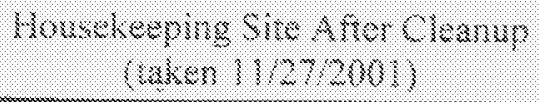 \\
\hline
\end{tabular}

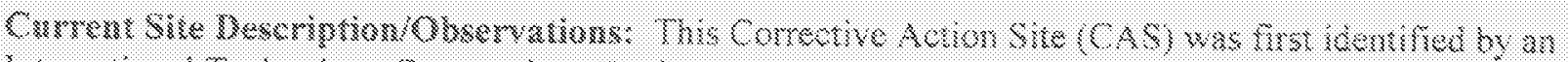

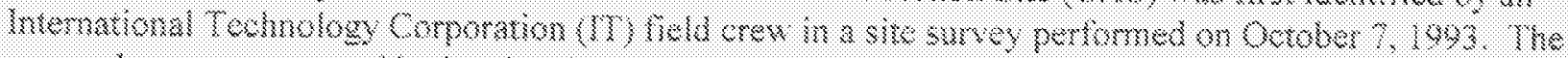

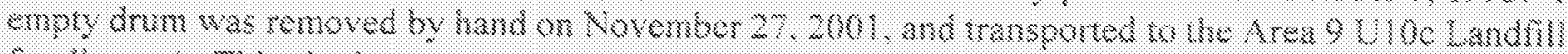

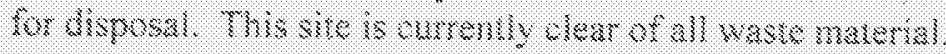

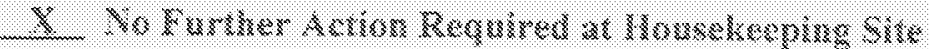

Yarsess Mixis

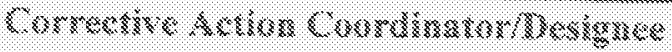

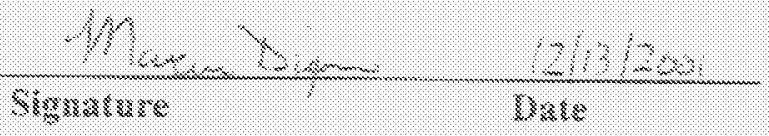




\section{Sectored Housekeeping Site Closure Verification Form}

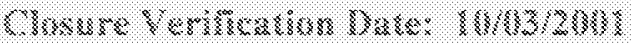

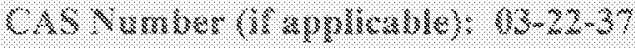

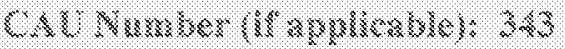

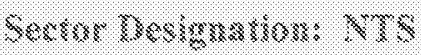

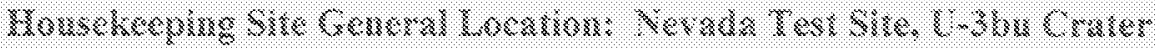

Elevarion: $122: 5$ maters $(\mathrm{m})$

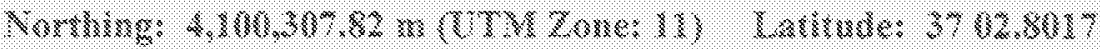

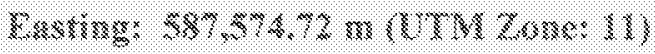

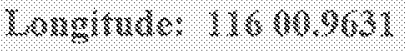

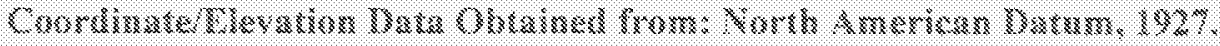

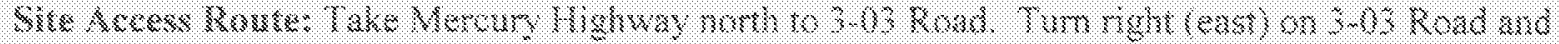

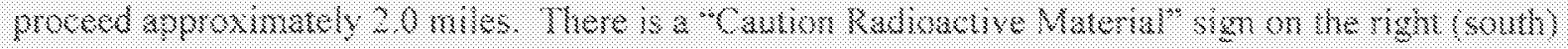

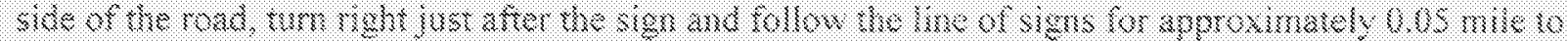

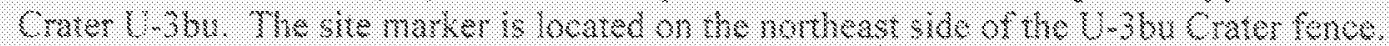

\begin{tabular}{|c|c|}
\hline Waste Krem(s) Originalit ast Site & Apprarens: Waste Type* \\
\hline 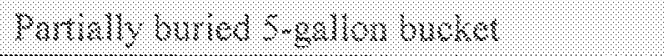 & Oromiary \\
\hline
\end{tabular}

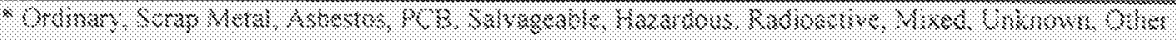

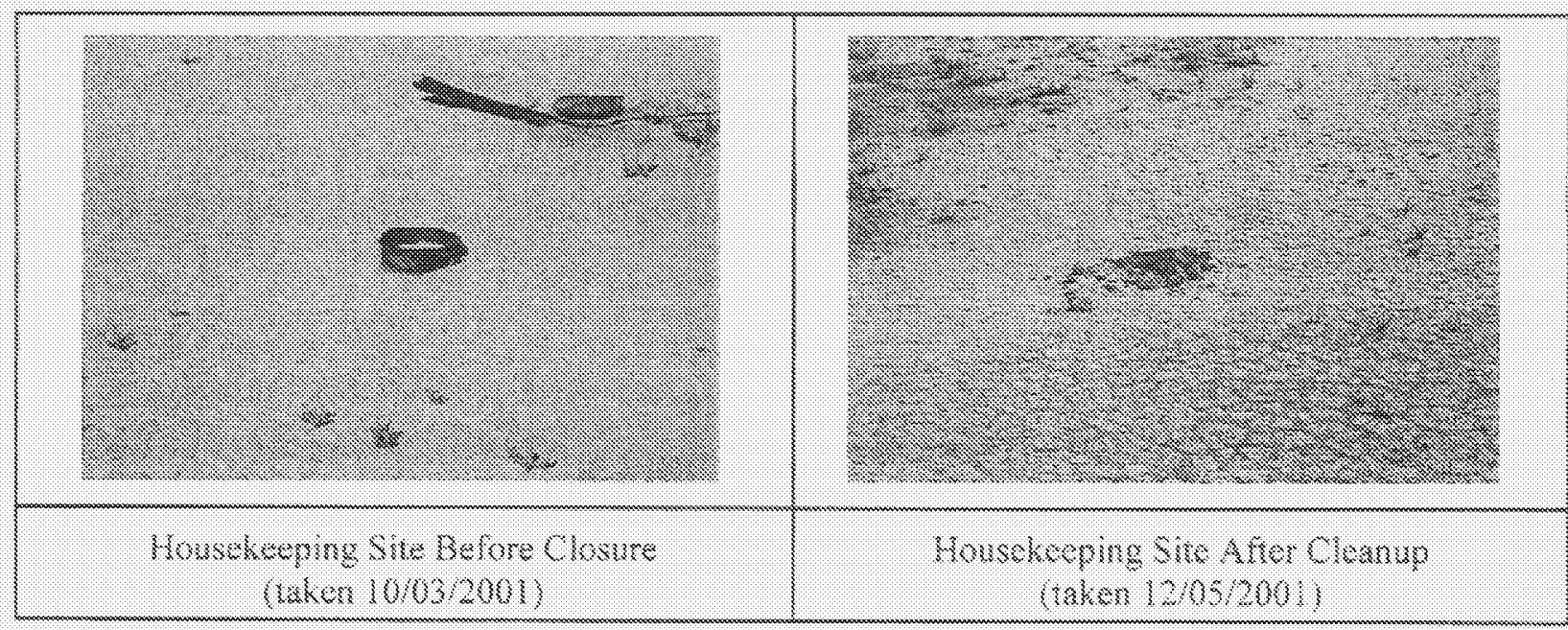

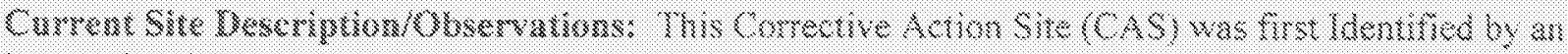

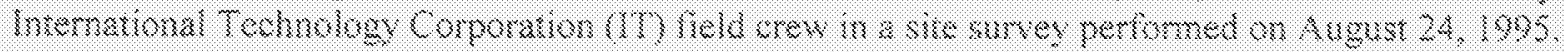

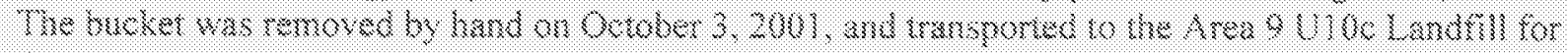

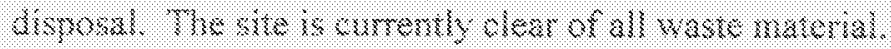

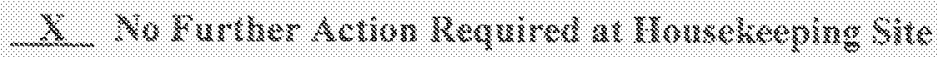

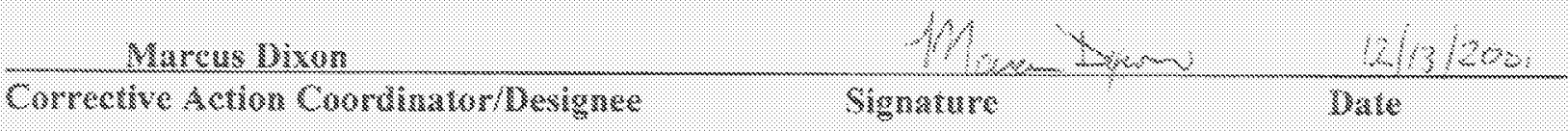




\section{Sectored Housekeeping Site Closure Verification Form}

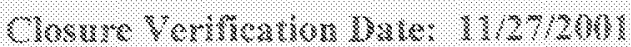

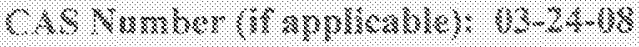

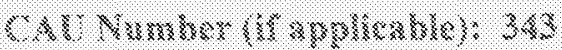

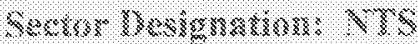

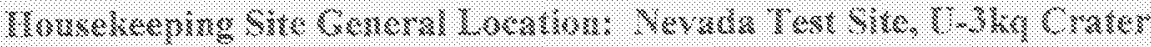

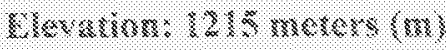

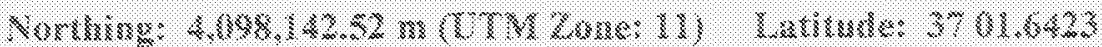

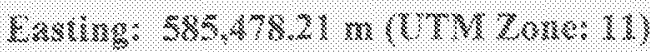

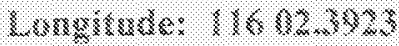

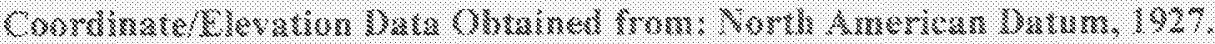

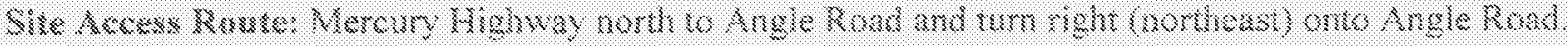

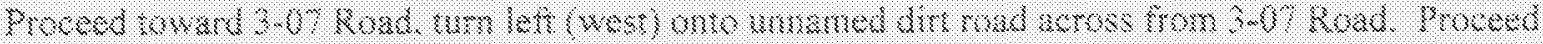

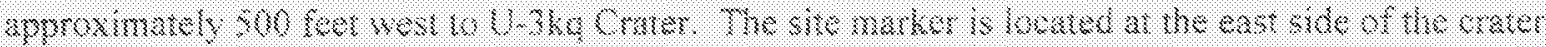
kerce

\begin{tabular}{|c|c|}
\hline 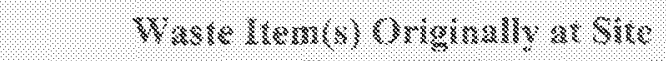 & 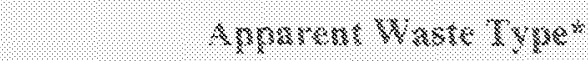 \\
\hline Batrem & Recowable \\
\hline
\end{tabular}

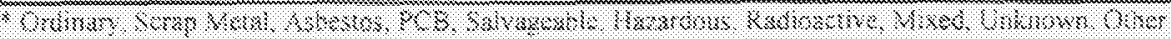

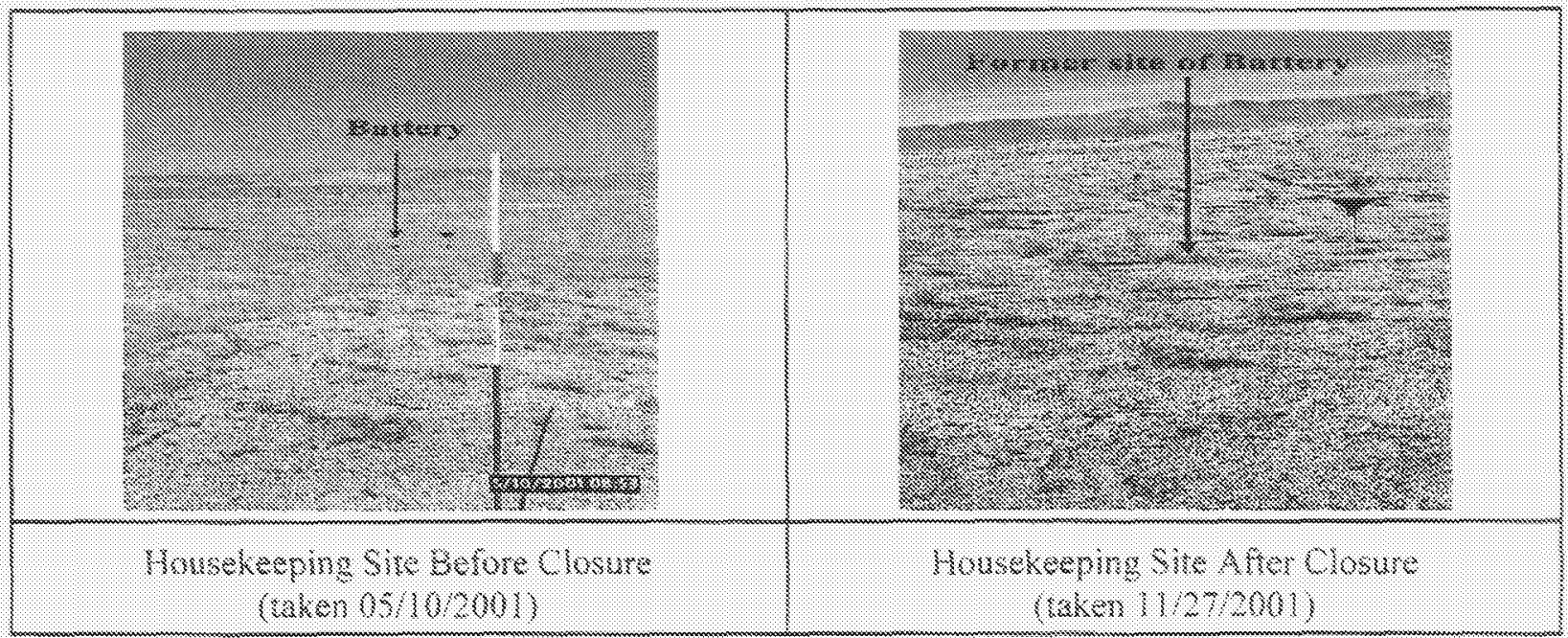

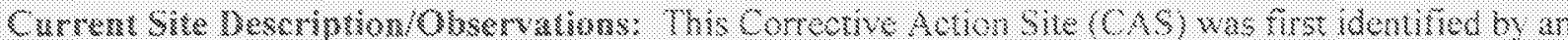

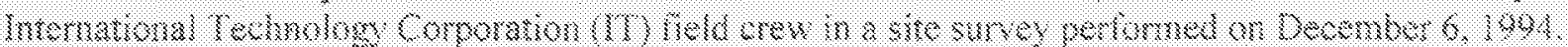

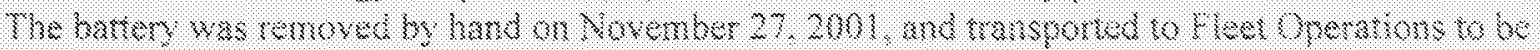

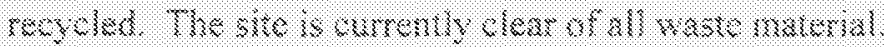

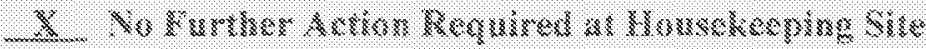

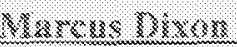

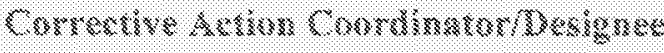

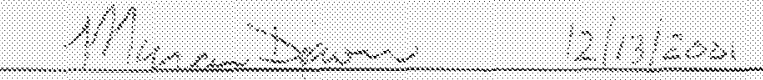




\section{Sectored Housekeeping Site Closure Verification Form}

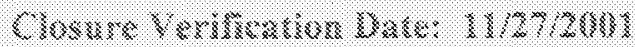

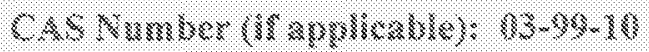

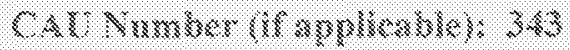

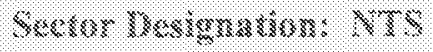

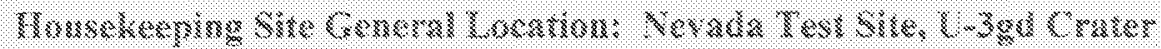

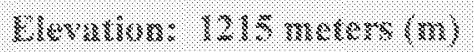

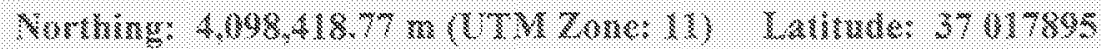

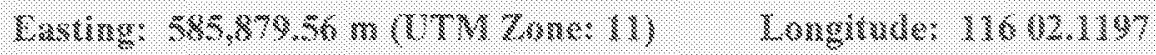

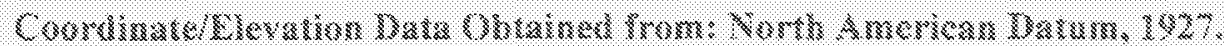

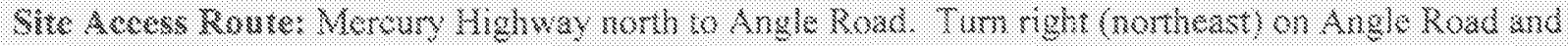

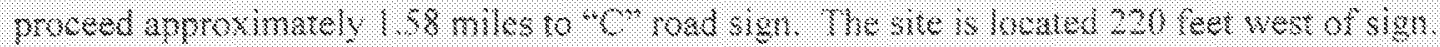

\begin{tabular}{|c|c|}
\hline 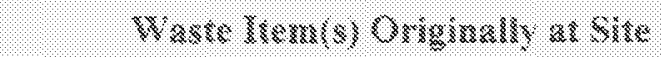 & 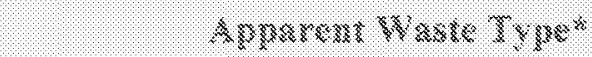 \\
\hline 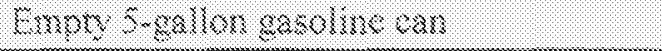 & (J) \\
\hline
\end{tabular}

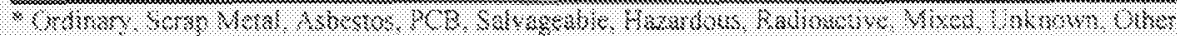

\begin{tabular}{|c|c|}
\hline 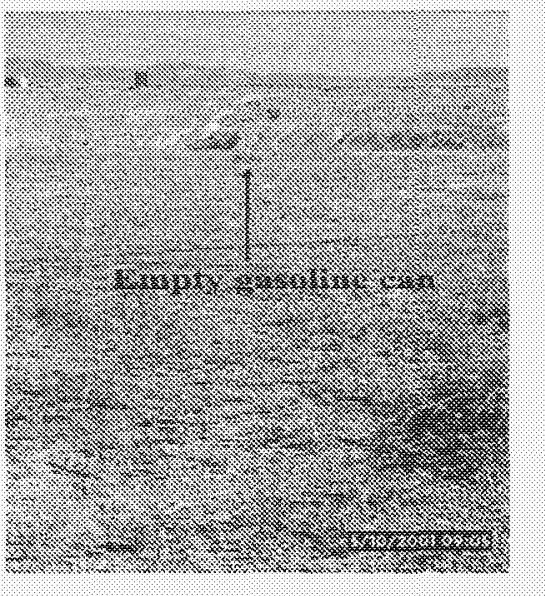 & 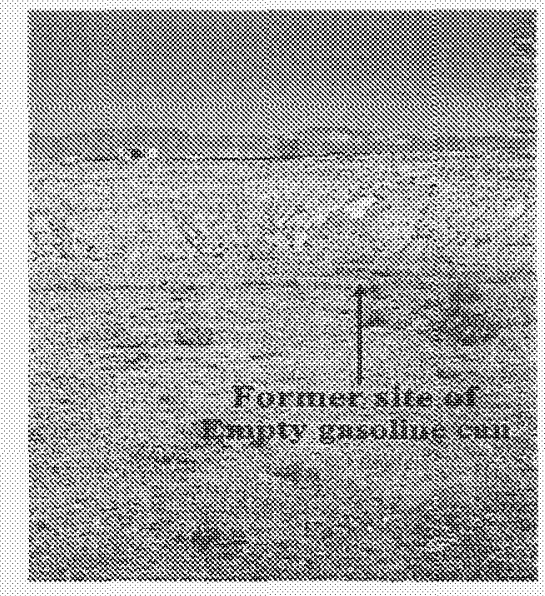 \\
\hline 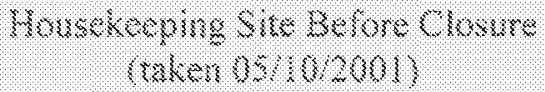 & 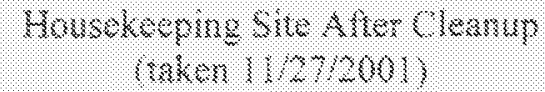 \\
\hline
\end{tabular}

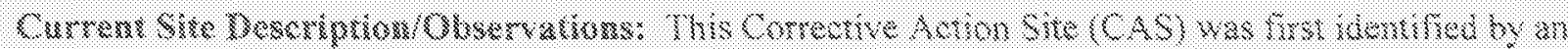

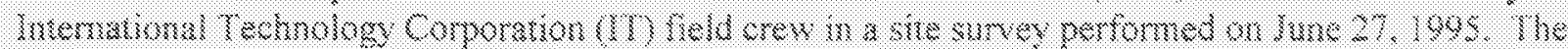

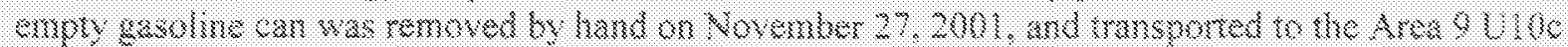

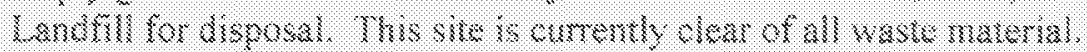

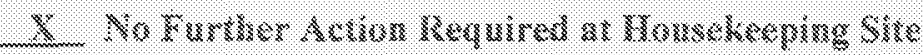

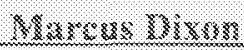

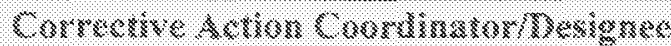

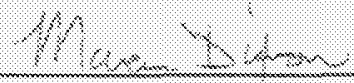

Signgature

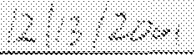

Braxte 


\section{APPENDIX C}

CAS SPECIFIC SAMPLING AND ANALYSIS PLAN 
THIS PAGE INTENTIONALLY LEFT BLANK 


\section{APPENDIX C TABLE OF CONTENTS}

Sample Collection Criteria $\ldots \ldots \ldots \ldots \ldots \ldots \ldots \ldots \ldots \ldots \ldots \ldots \ldots \ldots \ldots$

Verification Sampling and Analysis for CAS $01-24-03 \ldots \ldots \ldots \ldots \ldots \ldots \ldots \ldots$

Verification Sampling and Analysis for CAS $03-22-25 \ldots \ldots \ldots \ldots \ldots \ldots \ldots \ldots$

Verification Sampling and Analysis for CAS $03-22-14 \ldots \ldots \ldots \ldots \ldots \ldots \ldots$

Verification Sampling and Analysis for CAS $03-22-07 \ldots \ldots \ldots \ldots \ldots \ldots \ldots$ 
THIS PAGE INTENTIONALLY LEFT BLANK 


\section{SAMPLING STRATEGY FOR CORRECTIVE ACTION UNIT 343: AREAS 1, 3, \& 4 HOUSEKEEPING SITES}

Corrective Action Unit (CAU) 343 consists of 18 Corrective Action Sites (CAS) located in Areas 1,3 and 4 of the Nevada Test Site. However, only four sites require verification samples to show that all waste has been removed. Soil samples will be collected at each CAS from the surface using hand trowels.

All samples will be labeled with a unique sample number using the following nomenclature:

$032207+0-\mathrm{V}$

where:

032207 is the CAS number

0 is the sample depth in feet

$V$ represents type of sample

Quality control samples (blind replicates) will be collected. They will be labeled with their own distinct sample number so that the laboratory will not be able to identify them as quality control samples.

All samples will be collected with disposable sampling scoops and placed in appropriate sample containers. PPE involved with characterization sampling will include steel-toed boots, safety glasses, chemical resistant gloves and/or work gloves. Hard hats are required when overhead hazards exist. The disposable sampling scoops and latex gloves will be placed into the trash so decontamination will not be an issue. Samples will be placed on ice in coolers and transported under chain-of-custody to Environmental Technical Services in Mercury, Nevada for shipment to an off-site laboratory. Samples may be transported directly to the analytical laboratory by BN ER personnel during weekends or off-hours.

Soil samples will be preserved by cooling to $4 \mathrm{C}$. Trip blanks will be preserved with hydrochloric acid to a $\mathrm{pH}<2$, then cooled to $4^{\circ} \mathrm{C}$.

A Chain-of-Custody form will be filled out for each sample batch prior to shipment. Additionally, a Radiological Clearance Form (Green Tag) will be issued for each sample batch for shipment within and off the NTS.

The following tables show the sampling parameters for each CAS. 


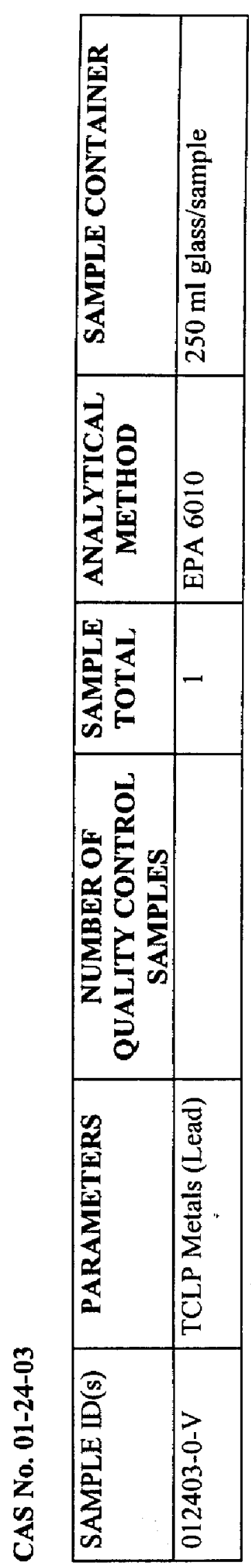

ปี 


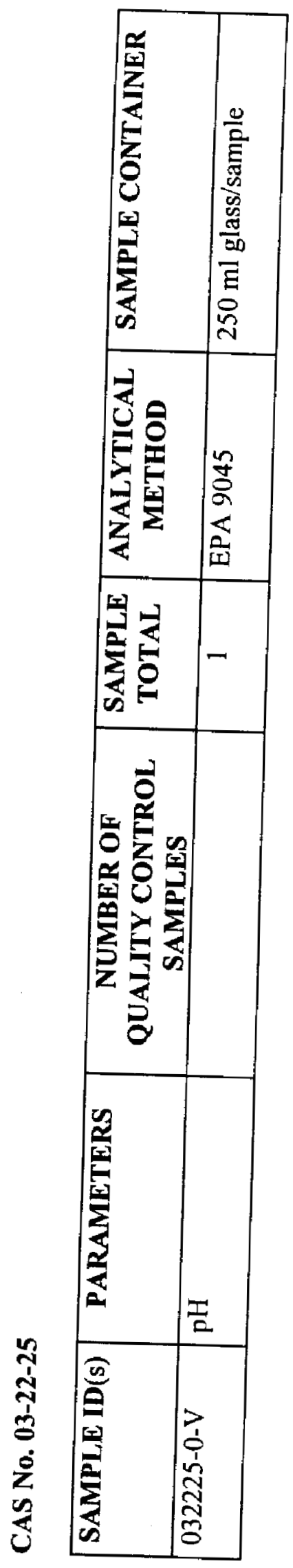

8 


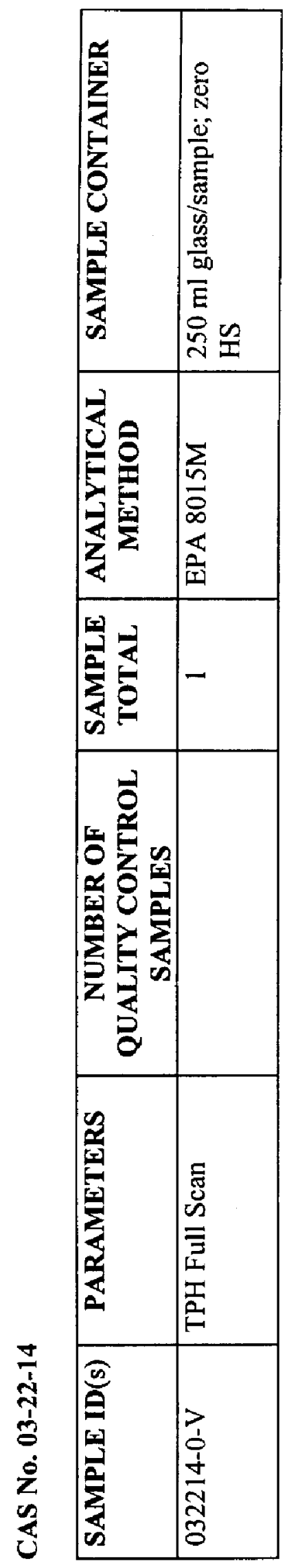

U 


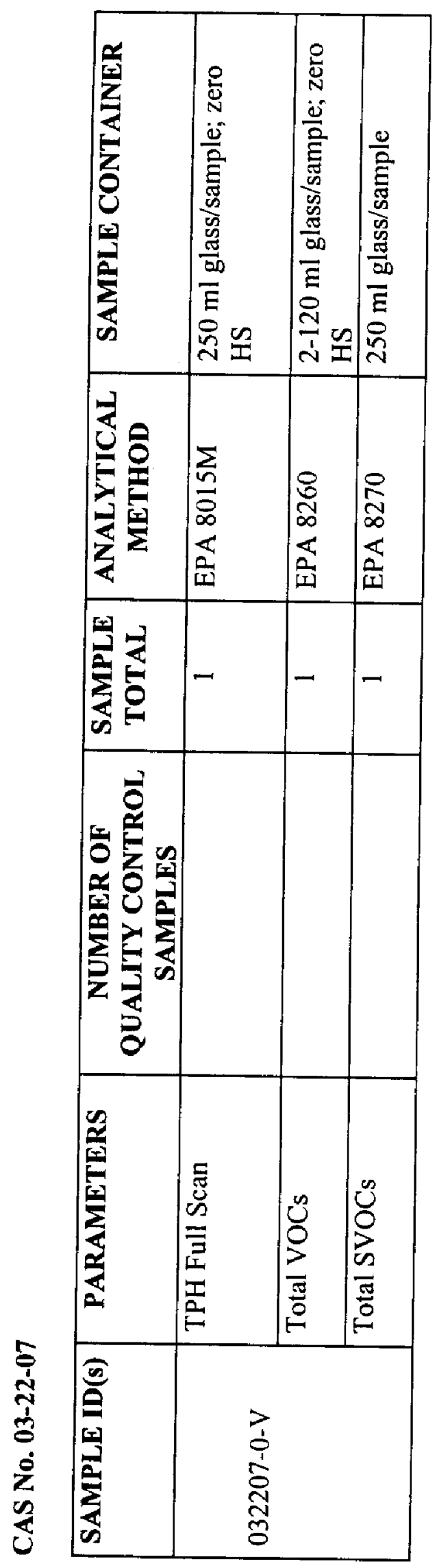

¿ 
THIS PAGE INTENTIONALLY LEFT BLANK

C-6 


\section{APPENDIX D \\ COMMENT RESPONSE DOCUMENTATION}


THIS PAGE INTENTIONALLY LEFT BLANK 


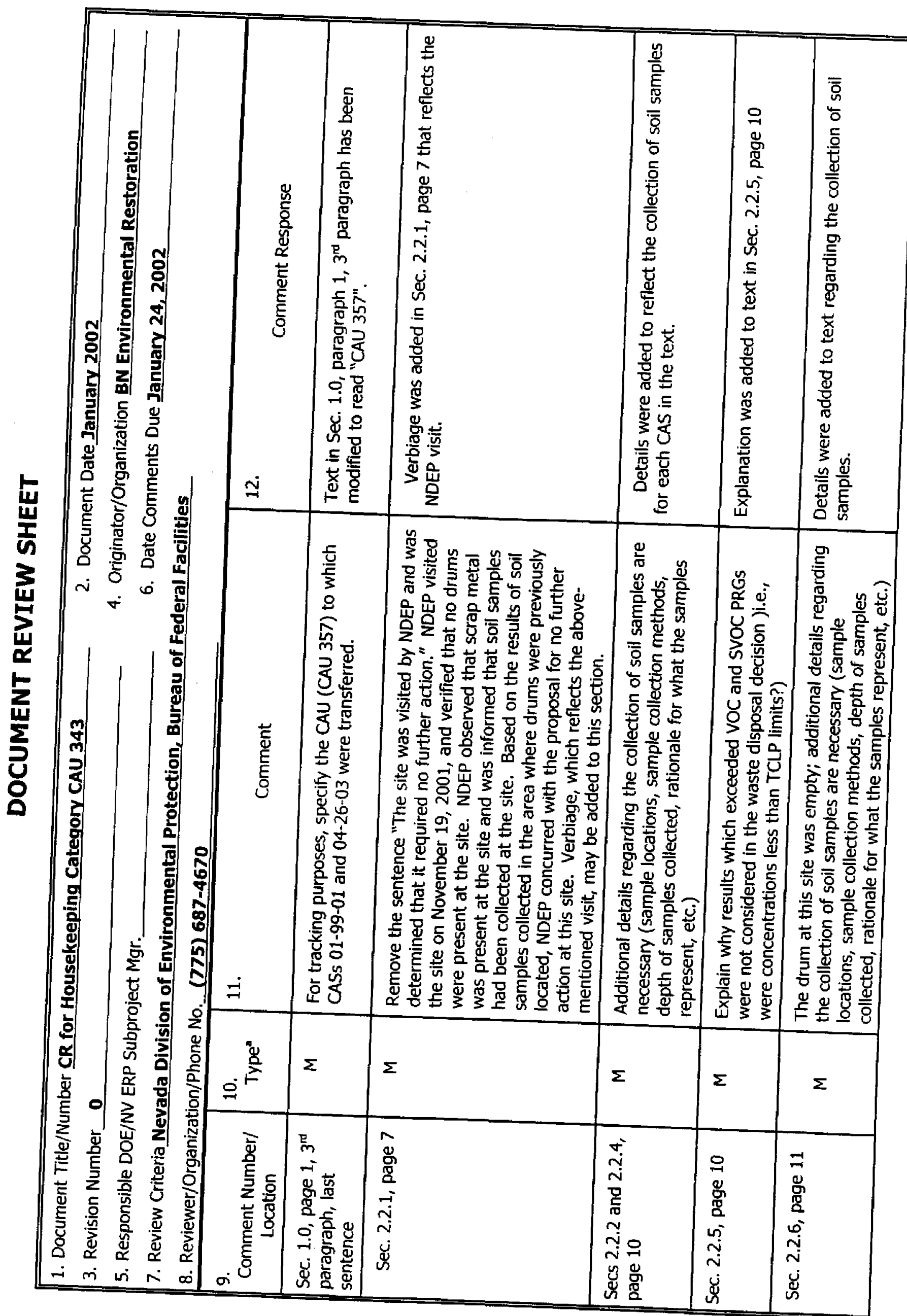




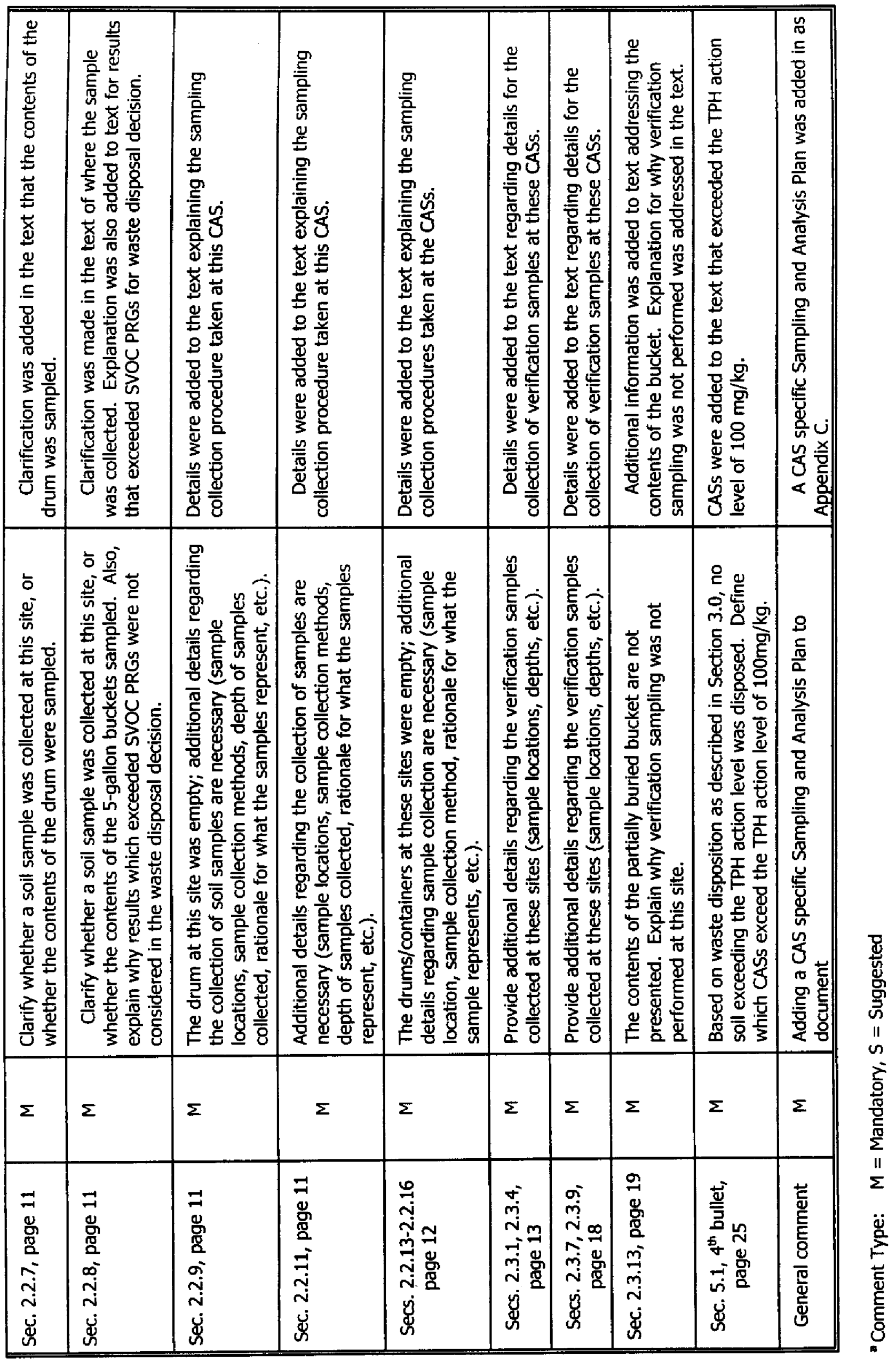




\section{DISTRIBUTION LIST}


THIS PAGE INTENTIONALLY LEFT BLANK 


\section{DISTRIBUTION LIST}

*Provide copy of initial distribution of Revision 0 ; remainder of list gets Revision 0 if approved without changes. The entire list receives Revision 1, if issued.

\section{Nevada Department of Environmental Protection}

Paul Liebendorfer

Bureau of Federal Facilities

Division of Environmental Protection

333 W. Nye Lane, Room 138

Carson City, NV 89706-0866

Supervisor, Las Vegas Office

Bureau of Federal Facilities

Division of Environmental Protection

555 E. Washington, Suite 4300

Las Vegas, NV 89010-1043

\section{U.S. Department of Energy}

Janet Appenzeller-Wing

Environmental Restoration Division

U.S. Department of Energy

National Nuclear Security Administration

Nevada Operations Office

P.O. Box 98518 , M/S 505

Las Vegas, NV 89193-8518

Sean Kosinski

Environmental Restoration Division

U.S. Department of Energy

National Nuclear Security Administration

Nevada Operations Office

P.O. Box 98518 , M/S 505

Las Vegas, NV 89193-8518

Sabrina Lawrence

Environmental Restoration Division

U.S. Department of Energy

National Nuclear Security Administration

Nevada Operations Office

P.O. Box 98518, M/S 505

Las Vegas, NV 89193-8518
2 (Controlled) $^{*}$

1 (Controlled)*

I (Uncontrolled)*

1 (Uncontrolled)*

1 (Controlled)* 


\section{DISTRIBUTION LIST (continued)}

\section{U.S. Department of Energy (continued)}

U.S. Department of Energy

National Nuclear Security Administration

Nevada Operations Office

Public Reading Facility

P.O. Box 98521 , M/S NLV040

Las Vegas, NV 89193-8521

U.S. Department of Energy

National Nuclear Security Administration

Nevada Operations Office

Technical Information Resource Center

P.O. Box $98518, \mathrm{M} / \mathrm{S} 505$

Las Vegas, NV 89193-8518

U.S. Department of Energy

Office of Scientific and Technical Information

P.O. Box 62

Oak Ridge, TN 37831-0062

\section{Bechtel Nevada}

Correspondence Control

Bechtel Nevada

P.O. Box 98521, M/S NLV008

Las Vegas, NV 89193-8521

Environmental Management Library

Bechtel Nevada

P.O. Box 98521, M/S NLV080

Las Vegas, NV 89193-8521

Marcus Dixon

Bechtel Nevada

P.O. Box 98521 , M/S NTS306

Las Vegas, NV 89193-8521

Ann Heidema

Bechtel Nevada

P.O. Box 98521 , M/S NLV022

Las Vegas, NV 89193-8521
1 (Controlled \&

1 Uncontrolled)

1 (Uncontrolled)

1 (electronic copy)
1 (Uncontrolled)*

1 (Uncontrolled)*

1 (Uncontrolled) 


\section{DISTRIBUTION LIST (continued)}

\section{Bechtel Nevada (continued)}

Thomas Fitzmaurice

1 (Uncontrolled)*

Bechtel Nevada

P.O. Box 98521 , M/S NTS306

Las Vegas, NV 89193-8521

Wayne Johnson

1 (Uncontrolled)*

Bechtel Nevada

P.O. Box 98521, M/S NTS306

Las Vegas, NV 89193-8521

Steve Nacht

Bechtel Nevada

P.O. Box $98521, \mathrm{M} / \mathrm{S}$ NTS306

Las Vegas, NV 89193-8521

\section{IT Corporation}

Lynn Kidman

IT Corporation

P.O. Box 93838, M/S 439

Las Vegas, NV 89193-8521

Garry Romano

IT FFACO Support Office

IT Corporation

1 (Uncontrolled)*

P.O. Box 93838, M/S 439

Las Vegas, NV 89193-8521

\section{State of Nevada}

Manager, Northern Nevada

1 (Uncontrolled)*

FFACO Public Reading Facility

1 (Uncontrolled)

Nevada State Library and Archives Federal Publications

100 North Stewart Street

Carson City, NV 89701-4285 
CLOSURE REPORT - CAU 343

Areas 1, 3, \& 4 Housekeeping Sites

Section: Distribution

Date: February 2002

THIS PAGE INTENTIONALLY LEFT BLANK 